\title{
Webs of rational curves on real surfaces and a classification of real weak del Pezzo surfaces
}

\author{
Niels Lubbes
}

March 9, 2021

\begin{abstract}
We classify webs of minimal degree rational curves on surfaces and give a criterion for webs being hexagonal. In addition, we classify Neron-Severi lattices of real weak del Pezzo surfaces. These two classifications are related to root subsystems of E8.
\end{abstract}

Keywords: families of curves, hexagonal webs, real weak del Pezzo surfaces, Neron-Severi lattice, linear series, root systems

MSC2010: 14C21, 14D99, 14Q10, 14P99, 53A60

\section{Contents}

1 Introduction $\quad 2$

2 Preliminaries $\quad 9$

3 Divisor classes and weak del Pezzo surfaces 12

4 Cremona invariant for weak del Pezzo surfaces 19

5 A classification of weak del Pezzo surfaces 22

6 Simple family graphs via adjunction 30

$\begin{array}{lll}7 & \text { A characterization of hexagonal webs } & 38\end{array}$

8 Acknowledgements $\quad 53$

9 References $\quad 53$ 


\section{Introduction}

Lines play a central role in Euclidean geometry and are rational curves of minimal degree. Through any two points in the plane exists a unique line and the family of lines in the plane is 2-dimensional. We investigate the geometry of real surfaces in projective space, by considering surfaces as a union of curves that are "simple". With surface we shall mean a real irreducible algebraic surface (see §2). A simple family is an algebraic family of minimal degree rational curves that covers a surface $X \subset \mathbb{P}^{n}$, such that a general curve in this family is smooth outside the singular locus of $X$. Moreover, we assume that the dimension of a simple family is as large as possible (see Definition 3). A simple curve is a curve that belongs to some simple family.

The intersection product of two simple families that cover $X$ is defined as the number of intersections between a general curve in the first family and a general curve in the second family, outside the singular locus of $X$.

The simple family graph $\mathcal{G}(X)$ is defined as follows:

- Each vertex is a simple family of $X$. A vertex is labeled with the dimension of the simple family.

- We draw between two simple families an edge if their intersection product is at least two. Such an edge is labeled with this intersection product.

Notice that simple families in $\mathcal{G}(X)$ that are not connected by an edge must have intersection product one.

See Figure 1 for examples of simple family graphs. The simple family graph of a one-sheeted hyperboloid was discovered in 1669 by Sir Christopher Wren [35]. The simple family graph of a ring cyclide was discovered by Yvon Villarceau and the two neighboring vertices define families of Villarceau circles [32, 1848]. The simple family graph of a Blum cyclide was discovered in 1980 by Richard Blum [3]. The ring cyclide, Blum cyclide and dP6 are examples of weak del Pezzo surfaces of degree 4, 4 and 6, respectively (see Definition 4).

In this article we address the following problem.

Problem 1. Classify simple family graphs $\mathcal{G}(X)$ of real surfaces $X \subset \mathbb{P}^{n}$ and determine properties of $X$ that are encoded in the invariant $\mathcal{G}(X)$. 
In the following Theorem $1,[\Lambda]^{2}$ denotes the set of 2-element subsets of $\Lambda:=$ $\{1, \ldots, m\}$ for some $m \geq 3$. If $G$ and $H$ are non-empty graphs, then $G \diamond H$ is the graph that is defined as the union $G \cup H$, where in addition each vertex in $G$ is connected with each vertex in $H$ via an edge with label 2 .

Theorem 1. If $\mathcal{G}(X)$ is the simple family graph of a real surface $X \subset \mathbb{P}^{n}$, then either $\mathcal{G}(X)=\emptyset$ or $\mathcal{G}(X)$ is characterized by one of the following graphs:

a. The minimal family graph of a real weak del Pezzo surface which is encoded by a row in Table 7 and where each vertex has label 1.

b. The graph that consists of a single vertex with label 3.

c. The graph that consists of a single vertex with label 2.

d. A graph that consists of vertices labeled 1 and no edges.

e. A completely connected graph such that each vertex has label 1 and each edge has label 2.

f. The graph with vertex set $[\Lambda]^{2}$ such that each vertex has label 1 and an edge between different vertices $A, B \in[\Lambda]^{2}$ has label $4-2|A \cap B|$.

g. The graph with vertex set $[\Lambda]^{2}$ such that there is an edge between different vertices $A, B \in[\Lambda]^{2}$ if and only if $|A \cap B|=0$. Each vertex has label 1 and each edge has label 2.

h. $G \diamond H$ where $G$ and $H$ are characterized by (d) and (e), respectively.

i. $G \diamond H$ where $G$ and $H$ are characterized by (d) and (f), respectively.

j. $G \diamond H$ where $G$ and $H$ are characterized by (e) and (g), respectively.

k. $G \diamond H$ where $G$ and $H$ are characterized by (c) and (e), respectively.

l. $G \diamond H$ where $G$ and $H$ are characterized by (c) and (f), respectively; optionally, some vertices with label 1 are omitted.

Corollary 1. Suppose that $X \subset \mathbb{P}^{n}$ is a real surface. A vertex in $\mathcal{G}(X)$ has label $\leq 3$ and an edge in $\mathcal{G}(X)$ has label $\leq 8$. If an edge has label 3 or label $\geq 5$, then $\mathcal{G}(X)$ contains $\leq 2160$ vertices and $\leq 2262600$ edges. 


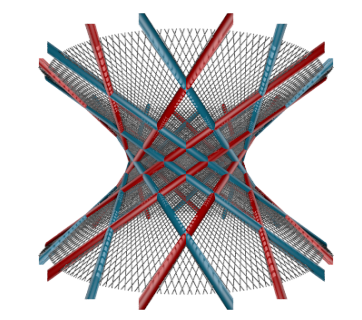

(1) (1)

one-sheeted hyperboloid

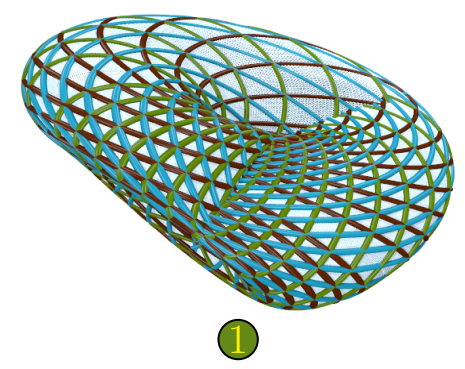

(1) (1)

dP6
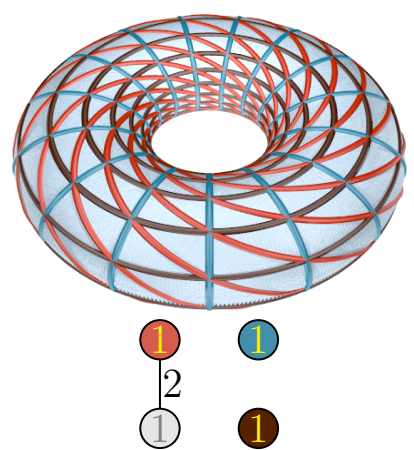

ring cyclide

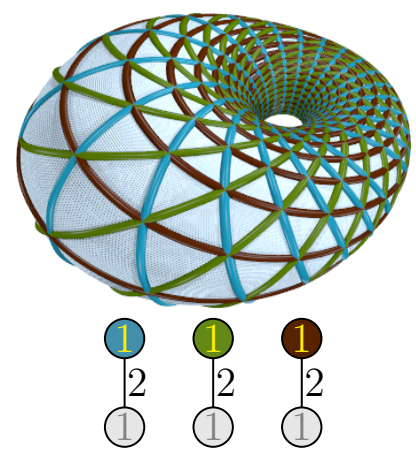

Blum cyclide

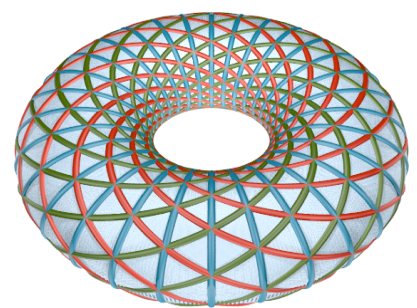

(1) (1) 2
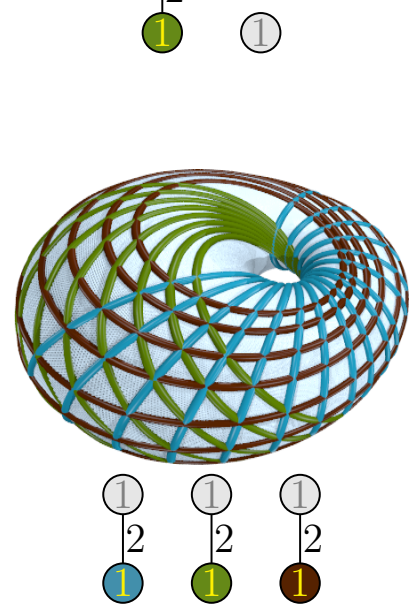

Figure 1: Examples of simple family graphs.

Corollary 2. If $\mathcal{G}(X)$ has $v \geq 4$ vertices and is characterized by Theorem 1 a, then $\mathcal{G}(X)$ is not characterized by Theorem 1[b-l], except for the simple family graphs associated to the following rows in Table \%:

- $v=4$ : rows $\{16,33\},\{116\}$ and $\{283,284,288,289,647,671\}$ are also characterized by Theorem 1d, Theorem 1 and Theorem 1e, respectively.

- $v=5$ : row 15 is also characterized by Theorem $1 d$.

- $v=6$ : rows 335, 360, 708, 714, 721, 737, 745 and 761 are also characterized by Theorem $1 f$.

- $v=7$ : rows 267, 268 and 272 are also characterized by Theorem 1 .

The proof of Theorem 1 uses results from [18] (see Lemma 9 and Lemma 10). We recover [18, Corollary 1a] which addresses the second part of Problem 1:

Corollary 3. Suppose that $X \subset \mathbb{P}^{n}$ is a real surface. If a vertex in $\mathcal{G}(X)$ has label $\geq 3$, then the normalization of $X$ is biregular isomorphic to a quadric whose real points form the unit-sphere $S^{2} \subset \mathbb{R}^{3}$. 
The unique recovery of $\mathcal{G}(X)$ from a row in Table 7 will be discussed at Theorem 2 in a moment and later at Example 2. Our methods are constructive and we hope to interest both geometric modelers and algebraic geometers. With [18, Algorithm 1] we can compute the graph $\mathcal{G}(X)$ from a given birational map $\mathbb{P}^{2} \rightarrow X$. The

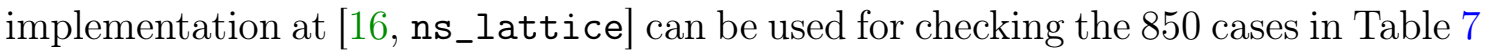
automatically. We find for example that a simple family graph does not consist of 4 vertices and 2 edges. See [17, orbital] for the visualization of simple families on surfaces.

The Cayley-Salmon theorem states that a smooth cubic surface over an algebraically closed field contains 27 straight lines [4, 1848] and the intersection graph of the lines is the dual of the Schläfli graph. The set of hyperplane sections of the cubic surface containing at least one of these lines define 27 families of conics. In the cubic Clebsch surface these 27 families are real $[5,1871]$ and the corresponding simple family graph is in Figure 2.

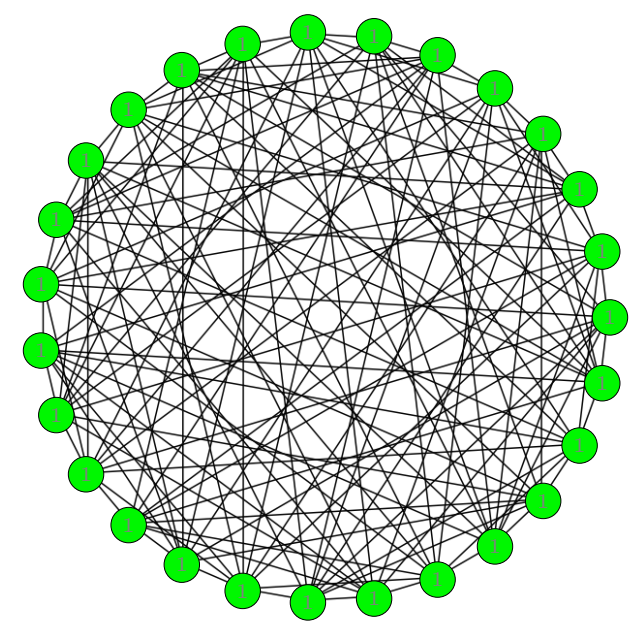

Figure 2: The 10-regular graph with 27 vertices is the simple family graph of a Clebsch surface (the edges are labeled 2). This graph is known as the generalized quadrangle $G Q(2,4)$ [34].

Normal cubic surfaces are special cases of weak del Pezzo surfaces [6, 19]. Intersection graphs of lines on (complex) del Pezzo surfaces have been studied [20] and these intersection graphs uniquely determine the simple family graphs of the surfaces. An advantage of simple family graphs of weak del Pezzo surfaces is that, unlike intersection graphs of lines, such graphs apply to all rational surfaces whose simple family graphs are not characterized by one of the eleven "exceptional graph types" as listed in Theorem 1[b-l]. 
Del Pezzo surfaces are investigated since at least 1830 [6, Chapter 9, Historical notes] and the geometry of these celebrated surfaces remain more than 175 years later still a topic of research $[24,26]$. Del Pezzo surfaces play a central role in the classification of real rational surfaces [14, Theorem 1.9][30, Corollary 2.6]. See [28] for an introduction to the theory of del Pezzo surfaces with emphasis on geometric modeling.

The Neron-Severi lattice $\mathbf{N}(X)$ of a weak del Pezzo surface $X \subset \mathbb{P}^{n}$ encodes to a large extend the geometry of $X$ and in particular its minimal family graph $\mathcal{G}(X)$ (see Proposition 2 and Remark 3). It is remarkable that this algebraic structure is determined by the combinatorics of the E8 root system. See Definition 1 for the data associated to the Neron-Severi lattice and see Definition 4 for the notion of weak del Pezzo surface. In light of Theorem 1a we classify the Neron-Severi lattices of real weak del Pezzo surfaces.

Theorem 2. If $X$ is a real weak del Pezzo surface, then its Neron-Severi lattice $\mathbf{N}(X)$ is up to isomorphism uniquely characterized by exactly one of 825 rows in Table $\%$.

See Example 2 for an explanation of how to read Table 7 and how to recover $\mathcal{G}(X)$ uniquely from a row. Theorem 2 has been known before with the following additional assumptions:

- $X$ is a real (non-weak) del Pezzo surface [33, Corollary 2.1].

- the real structure of $X$ acts trivially on $N(X)$ [7] (see also [6, Sections 8.7.1 and 8.8.1]).

- $X$ is a real weak del Pezzo surface of degree 3 with only real singularities [13] (there are 44 cases).

If $X \subset \mathbb{P}^{3}$ is quadric surface, then $\mathbf{N}(X)$ is isomorphic to the Neron-Severi lattice of either a sphere, a quadric cone or a hyperboloid of one-sheet. In comparison, if $X \subset \mathbb{P}^{3}$ is a cubic surface with at most isolated singularities, then it follows from Theorem 2 that there are up to isomorphism 56 different choices for $\mathbf{N}(X)$ and the number of real lines in $X$ is either $\leq 12$ or in $\{15,16,21,27\}$.

A $\lambda$-web of curves on a surface $X$ is defined as a set $\mathcal{W}$ of algebraic curves contained in $X$ such that through almost every point in $X$ pass exactly $\lambda$ curves in $\mathcal{W}$. Something remarkable can be observed about the 3 -webs of simple curves as visualized 
in Figure 1. In 1927, Thomson and Blaschke talked about such webs during their spring walks on Posillipo hill in Italy [2, Vorwort]. The observation is that an open space on the surface is bordered by exactly three simple curves in a discretized realization of the web. In particular, many hexagonal patterns emerge and therefore such triangular 3-webs are also known as hexagonal webs (see Definition 6 or [8, Lecture 18]). Hexagonal webs of simple curves lead to nice triangularizations of the underlying surface. The property for a web to be hexagonal is purely topological, and its existence reveals in some cases properties of the surface under study. See [22, Appendix] for an overview of historical and recent developments in web geometry. For understanding the geometry of hexagonal webs of simple curves let us first consider $\mathbb{P}^{2}$ (Figure 3 ). In this case, simple curves are exactly the lines and $\mathcal{G}\left(\mathbb{P}^{2}\right)$ is characterized by Theorem 1c. Such webs are of recent interest in combinatorial geometry [10, Figure 14]. Hexagonal webs of lines are characterized in the following theorem from [9, 1924] (see also [2, Section 1.3, page 24]).
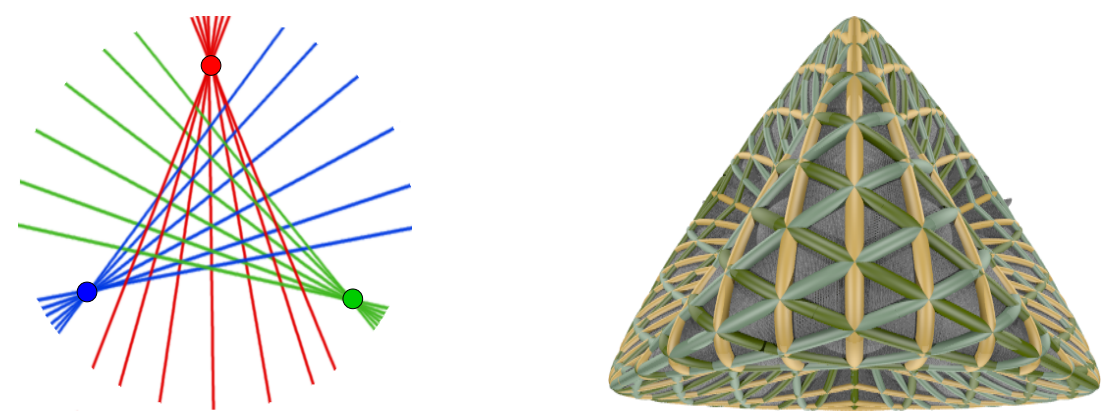

Figure 3: On the left we see that three pencils of lines form a hexagonal web. The three pencils correspond to a reducible cubic consisting of three lines in the dual plane. The Roman surface on the right is a linear projection to $\mathbb{P}^{3}$ of a Veronese embedding of the planar web into $\mathbb{P}^{5}$.

Theorem A (Graf-Sauer, 1924). A 3-web of lines in $\mathbb{P}^{2}$ form a hexagonal web if and only if the lines correspond to points on a cubic curve in the dual plane $\mathbb{P}^{2 *}$.

The next surface one might investigate is the projective closure $\mathbb{S}^{2} \subset \mathbb{P}^{3}$ of the unit sphere $S^{2} \subset \mathbb{R}^{3}$. The simple curves on $\mathbb{S}^{2}$ are circles and $\mathcal{G}\left(\mathbb{S}^{2}\right)$ is characterized by Theorem 1b. The classification problem for hexagonal webs of circles is known as the Blaschke-Bol problem [2, §3, Aufgabe 1, page 31]. The general classification might be a very difficult problem, but some recent progress has been made [1, 21, 29]. 
A Darboux cyclide is a quartic weak del Pezzo surface in $\mathbb{S}^{3}$. For example, the Blum cyclide and ring torus in Figure 1 are stereographics projections of Darboux cyclides. Hexagonal 3-webs of circles on Darboux cyclides have recently been classified in [25, Theorem 17]. We translated this theorem to our setting:

Theorem B (Pottmann-Shi-Skopenkov, 2012). Suppose that $X \subset \mathbb{S}^{3}$ is a Darboux cyclide. Three vertices in $\mathcal{G}(X)$ define a hexagonal web if and only if either the vertices do not share an edge in $\mathcal{G}(X)$, or one of the vertices is isolated in $\mathcal{G}(X)$.

We remark that "three vertices do not share an edge" means that no two of the three vertices are connected by an edge. In this article we prove a generalization of the above theorem to any (real algebraic irreducible) surface.

Theorem 3. Let $X \subset \mathbb{P}^{n}$ be a real surface. If three vertices in $\mathcal{G}(X)$ do not share an edge, then their corresponding three simple families are 1-dimensional and form a hexagonal web.

We assume that an embedded surface $X \subseteq \mathbb{P}^{n}$ is not contained in a hyperplane section. The following corollary addresses the Blaschke-Bol problem:

Corollary 4. If $X \subseteq \mathbb{P}^{n}$ is a real surface that is covered by a hexagonal web of conics, then $X$ is (a linear projection of) one of the following:

a. plane with $n=2$.

b. quadric surface with $n=3$.

c. cubic ruled surface with $3 \leq n \leq 4$.

d. quartic Veronese surface with $3 \leq n \leq 5$.

e. weak del Pezzo surface of degree $d$ such that $3 \leq n \leq d \leq 6$.

In Figure 1 we see hexagonal webs of circles covering weak del Pezzo surfaces of degree four and six. Figure 3 illustrates Corollary 4d.

Surface parametrizations whose parameter lines form simple families are of interest in geometric modeling:

Theorem 4. If $X \subset \mathbb{P}^{n}$ is a real surface such that $\mathcal{G}(X)$ contains two disjoint vertices, then there exists a real birational map $\mathbb{P}^{1} \times \mathbb{P}^{1} \rightarrow X$ of bidegree $(d, d)$, where $d$ is the degree of a simple curve on $X$. Moreover, if a real birational map $\mathbb{P}^{1} \times \mathbb{P}^{1} \rightarrow X$ is of bidegree $(a, b)$, then $a, b \geq d$. 
Notice that Corollary 3, Theorem 3 and Theorem 4 address the second part of Problem 1.

\section{Preliminaries}

A real variety $X$ is defined as a complex variety together with an antiholomorphic involution $\sigma: X \rightarrow X$ [30, Proposition I.1.3]. We call $\sigma$ the real structure of $X$. Curves, surfaces and projective spaces $\mathbb{P}^{n}$ are real varieties and maps between them are real, unless explicitly stated otherwise. In fact, all structures are compatible with the real structure unless explicitly stated otherwise. We call $X \mathbb{R}$-rational if there exists a birational map $\mathbb{P}^{2} \rightarrow X$.

A smooth model of a surface $X$ is a birational morphism $Y \rightarrow X$ from a nonsingular surface $Y$, such that this morphism does not contract complex $(-1)$-curves. This model exists and is unique up to biregular isomorphisms by [15, Theorem 2.16]. Therefore, we will henceforth refer to a smooth model of a surface as the smooth model.

Suppose that $Y \rightarrow X$ is the smooth model of a surface $X \subset \mathbb{P}^{n}$. A linear normalization $X_{N} \subset \mathbb{P}^{m}$ of $X$ is defined as the image of $Y$ via the map associated to the complete linear series of hyperplane sections of $X$. Thus, $m \geq n, X$ is a linear projection of $X_{N}$ and $X_{N}$ is unique up to $\operatorname{Aut}\left(\mathbb{P}^{m}\right)$.

Definition 1. The Neron-Severi lattice $\mathbf{N}(X)$ of a surface $X \subset \mathbb{P}^{n}$ with smooth model $\varphi: Y \rightarrow X$ consists of the following data:

1. A unimodular lattice $N(X)$ defined by divisor classes on $Y$ modulo numerical equivalence.

2. Two distinguished classes $h, k \in N(X)$ corresponding to the class of hyperplane sections and the canonical class of $Y$, respectively. The class $h$ is the divisor class of a curve $H \subset Y$ such that $\varphi(H)$ is a hyperplane section of $X$.

3. A unimodular involution $\sigma_{*}: N(X) \rightarrow N(X)$ induced by the real structure $\sigma: X \rightarrow X$.

4. A function $h^{0}: N(X) \rightarrow \mathbb{Z}_{\geq 0}$ assigning the dimension of global sections of the line bundle associated to a class. 
The class $[C] \in N(X)$ of a complex curve $C \subset X$ is defined as the divisor class of the 1-dimensional part of its complex preimage $\varphi^{-1}(C)$ minus the components that are contracted by the smooth model $\varphi: Y \rightarrow X$. Notice that $h$ is the class of a hyperplane section of $X$.

Definition 2. Suppose that $Y \rightarrow X$ is the smooth model of a surface $X$. We call a complex birational morphism $\pi: Y \rightarrow Y^{\prime}$ a sequential blowup if there exists complex blowups $\pi_{i}: Y_{i+1} \rightarrow Y_{i}$ with complex centers $p_{i} \in Y_{i}$ such that $Y=Y_{r+1}, Y^{\prime}=Y_{1}$ and $\pi=\pi_{1} \circ \ldots \circ \pi_{r}$. If $p_{i+1} \in Y_{i+1}$ lies on the complex $(-1)$-curve that contracts to $p_{i} \in Y_{i}$, then we say that $p_{i}$ is infinitely near to $p_{i+1}$. We call $\left(p_{1}, \ldots, p_{r}\right)$ a center of $\pi$ and we refer to $\left(c_{1}, \ldots, c_{r}\right)$ as exceptional classes of $\pi$ if $c_{i}$ is the pullback along $\pi_{i+1} \circ \ldots \circ \pi_{r}$ of the class of the complex $(-1)$-curve that contracts to $p_{i}$ for all $1 \leq i \leq r$.

Let $\Gamma_{1}:=\left\langle e_{0}, e_{1}, \ldots, e_{r}\right\rangle_{\mathbb{Z}}$ generate a unimodular lattice such that $e_{0}^{2}=1, e_{i}^{2}=-1$ and $e_{0} \cdot e_{j}=e_{i} \cdot e_{j}=0$ for $1 \leq i<j \leq r$. We call $\Gamma_{1}$ a type 1 basis for $N(X)$ if $N(X) \cong \Gamma_{1}$ and there exists a sequential blowup $\pi: Y \rightarrow \mathbb{P}^{2}$ with exceptional classes $\left(e_{1}, \ldots, e_{r}\right)$ such that $e_{0}$ is the pullback along $\pi$ of the class of a line in $\mathbb{P}^{2}$.

Let $\Gamma_{2}:=\left\langle\ell_{0}, \ell_{1}, \varepsilon_{1}, \ldots, \varepsilon_{r}\right\rangle_{\mathbb{Z}}$ generate a unimodular lattice such that $\ell_{0} \cdot \ell_{1}=1$, $\varepsilon_{i}^{2}=-1$ and $\ell_{0}^{2}=\ell_{1}^{2}=\ell_{0} \cdot \varepsilon_{i}=\ell_{1} \cdot \varepsilon_{i}=\varepsilon_{i} \cdot \varepsilon_{j}=0$ for $1 \leq i<j \leq r$. We call $\Gamma_{2}$ a type 2 basis for $N(X)$ if $N(X) \cong \Gamma_{2}$ and there exists a sequential blowup $\pi: Y \rightarrow \mathbb{P}^{1} \times \mathbb{P}^{1}$ with exceptional classes $\left(\varepsilon_{1}, \ldots, \varepsilon_{r}\right)$ such that $\ell_{0}$ and $\ell_{1}$ are the pullbacks along $\pi$ of the classes of the complex fibers of the projections of $\mathbb{P}^{1} \times \mathbb{P}^{1}$ to its first and second factor, respectively.

Suppose that $h \in N(X)$ is the class of hyperplane sections of $X$. We call a type 1 basis real if $\sigma_{*}\left(e_{0}\right)=e_{0}$ and $\sigma_{*}\left(\left\{e_{1}, \ldots, e_{r}\right\}\right)=\left\{e_{1}, \ldots, e_{r}\right\}$, and we require that if $h \cdot e_{i} \geq h \cdot e_{j}$ for $0<i, j \leq r$, then $i \leq j$. We call a type 2 basis real if $\sigma_{*}\left(\left\{\ell_{0}, \ell_{1}\right\}\right)=\left\{\ell_{0}, \ell_{1}\right\}$ and $\sigma_{*}\left(\left\{\varepsilon_{1}, \ldots, \varepsilon_{r}\right\}\right)=\left\{\varepsilon_{1}, \ldots, \varepsilon_{r}\right\}$, and we require that if $h \cdot \varepsilon_{i} \geq h \cdot \varepsilon_{j}$ for $0<i, j \leq r$, then $i \leq j$. Bases with real types will only be considered in $\S 6$.

Definition 3. Suppose that $X \subset \mathbb{P}^{n}$ is a surface, $B$ a smooth variety and $F \subset X \times B$ a divisor. We call $F$ a family of curves of $X$, or family for short, if the second projection $\pi_{2}: F \rightarrow B$ is dominant. A member of $F$, corresponding to $b \in B$, is defined as the curve $F_{b}:=\left(\pi_{1} \circ \pi_{2}^{-1}\right)(b) \subset X$. If $C, C^{\prime} \subset X$ are members of the same 
family, then $[C]=\left[C^{\prime}\right]$. The class of the family $F$ is defined as the class of any of its members and is denoted by $[F]$.

- We call $F$ covering if the first projection $\pi_{1}: F \rightarrow X$ is dominant.

- We call $F$ rational if a general member of $F$ has geometric genus 0 .

- The degree of $F$ is defined as the degree of any member with respect to the embedding $X \subset \mathbb{P}^{n}$. Equivalently, the degree of $F$ is $h \cdot[F]$, where $h \in N(X)$ denotes the class of hyperplane sections.

- We call $F$ minimal if $F$ is a rational covering family and of minimal degree with respect to all rational covering families of $X$.

- We call $F$ complete if there exists a curve $C \subset X$ such that the set $\left\{C^{\prime} \subset\right.$ $\left.X \mid\left[C^{\prime}\right]=[C]\right\}$ defines exactly the set of members of $F$. In other words, $F$ forms a complete linear series.

- We call $F$ simple if $F$ is both minimal and complete.

- The dimension of $F$ is defined as $\operatorname{dim} B$. If $F$ is complete, then $\operatorname{dim} F=$ $h^{0}([F])-1$.

Definition 4. We call a surface $X \subset \mathbb{P}^{n}$ a weak del Pezzo surface if $-k$ is nef and big and if $h=-\alpha k$ for some $\alpha \in \mathbb{Q}_{>0}$. If $-k$ is also ample, then we call $X$ a (non-weak) del Pezzo surface. The canonical degree of $X$ is defined as $k^{2}$.

Remark 1. Notice that we call a surface $X$ with smooth model $Y \rightarrow X$ a (weak) del Pezzo surface if and only if $Y$ is a (weak) del Pezzo surface as defined in [6, Definition 8.1.12 and Definition 8.1.18]. Thus any degree preserving linear projection of the anticanonical model of a (weak) del Pezzo surface is in our terminology again a (weak) del Pezzo surface.

Remark 2. The constant $\alpha$ in Definition 4 is characterized in [6, Theorem 8.3.2 and Section 8.4.1]: if $1 \leq k^{2} \leq 2$, then $\alpha \in \mathbb{Z}_{\geq 4-k^{2}}$, if $3 \leq k^{2} \leq 7$, then $\alpha \in \mathbb{Z}_{>0}$ and if $8 \leq k^{2} \leq 9$, then $\left(k^{2}-6\right) \alpha \in \mathbb{Z}_{>0}$.

Definition 5. Suppose $X \subset \mathbb{P}^{n}$ is a surface. We call a class $c \in N(X)$ indecomposable if $h^{0}(c)>0$ and there do not exists nonzero $a, b \in N(X)$ such that $c=a+b$ with both $h^{0}(a)>0$ and $h^{0}(b)>0$. The incidence diagram of a subset $U$ of a Neron-Severi lattice or inner product space is defined as a labeled graph

$$
\mathfrak{D}(U):=(U,\{(r, s, r \cdot s) \mid r, s \in U, r \cdot s \neq 0\})
$$


Throughout this article we will consider subsets of $N(X)$ as stated in Table 1 . We call $W \subseteq B(X)$ a component if its elements define the vertices of an irreducible component of the incidence diagram $\mathfrak{D}(B(X))$.

Notation 1. We will use the abbreviations (RR), (SD), (KV), (AF) and (HI) for Riemann-Roch theorem, Serre duality, Kawamata-Viehweg vanishing theorem, arithmetic genus formula and Hodge index theorem, respectively. Recall that if $X$ is a rational surface, then $(\mathrm{RR})$ states that $h^{0}(c)-h^{1}(c)+h^{2}(c)=\frac{1}{2}\left(c^{2}-c \cdot k\right)+1$ for $c \in N(X)$, where $k$ denotes the canonical class. Recall that (SD) states that $h^{2}(c)=h^{0}(k-c)$, thus if $-k$ is nef and $-k \cdot(k-c)<0$, then $h^{2}(c)=0$. If $c-k$ is nef and big, then $h^{1}(c)=h^{2}(c)=0$ by $(\mathrm{KV})$. With (AF) we mean the formula $p_{a}(c)=\frac{1}{2}\left(c^{2}+k \cdot c\right)+1$. Thus if $c$ is the class of a irreducible and reduced complex curve, then $c^{2}+k \cdot c \geq-2$ and $c^{2}+k \cdot c$ is even. Recall that if $b^{2}>0$ and $b \cdot c=0$ for some $b, c \in N(X)$ such that $c \neq 0$, then (HI) states that $c^{2}<0$. Notice that if $Y \rightarrow X$ is the smooth model of a surface $X \subset \mathbb{P}^{n}$, then we do intersection theory on $Y$ and not on $X$ itself.

\section{Divisor classes and weak del Pezzo surfaces}

Suppose that $X \subset \mathbb{P}^{n}$ is a weak del Pezzo surface. In this section we describe a correspondence between classes in $N(X)$ and the following geometric aspects on this surface: isolated singularities, unmovable curves and simple families. We start by considering the subsets $R(X), E(X), F(X) \subset N(X)$ as defined in Table 1 .

Proposition 1. Suppose that $X$ is a weak del Pezzo surface with canonical class $k$ such that $1 \leq k^{2} \leq 7$.

a) There exists a (non-real) type 1 basis $\left\langle e_{0}, e_{1}, \ldots, e_{r}\right\rangle_{\mathbb{Z}}$ for $N(X)$ such that $k=$ $-3 e_{0}+e_{1}+\ldots+e_{r}$ and $r=9-k^{2}$.

b) If $c \in N(X)$ such that $-k \cdot c=c^{2}=1$ or $c \in E(X) \cup F(X)$, then $h^{0}(c)>0$.

c) Up to permutation of $\left(e_{i}\right)_{i>0}$, an element of $R(X), E(X)$ and $F(X)$ is with respect to a type 1 basis in Table 2, Table 3 and Table 4, respectively. If $c \in N(X)$ such that $-k \cdot c=c^{2}=1$, then $c=-k$. 
Table 1: Distinguished subsets of the Neron-Severi lattice $N(X)$ of a weak del Pezzo surface X. See Proposition 2, Lemma 4 and Proposition 3.

$$
\begin{aligned}
R(X):= & \left\{c \in N(X) \mid k \cdot c=0 \text { and } c^{2}=-2\right\} \\
T(X):= & \left\{c \in R(X) \mid h^{0}(c)>0 \text { or } h^{0}(-c)>0\right\} \\
B(X):= & \{c \in T(X) \mid c \text { is indecomposable }\} \\
S(X):= & \left\{c \in R(X) \mid \sigma_{*}(c)=-c\right\} \\
A(X):= & \text { linear independent subset }\left(b_{i}\right)_{i} \subset S(X) \text { such that } \\
& S(X) \cap\left\{ \pm \Sigma \alpha_{i} b_{i} \mid \alpha_{i} \in \mathbb{Z}_{\geq 0}\right\}=S(X) \text { (if it exists) } \\
E(X):= & \left\{c \in N(X) \mid k \cdot c=-1 \text { and } c^{2}=-1\right\} \\
F(X):= & \left\{c \in N(X) \mid k \cdot c=-2 \text { and } c^{2}=0\right\} \\
E_{\star}(X):= & \{c \in E(X) \mid c \cdot r \geq 0 \text { for all } r \in B(X)\} \\
F_{\star}(X):= & \{c \in F(X) \mid c \cdot r \geq 0 \text { for all } r \in B(X)\} \\
E_{\mathbb{R}}(X):= & \left\{c \in E_{\star}(X) \mid \sigma_{*}(c)=c\right\} \\
F_{\mathbb{R}}(X):= & \left\{c \in F_{\star}(X) \mid \sigma_{*}(c)=c\right\} \\
G(X):= & \{c \in N(X) \mid c \text { is the class of a simple family of } X\}
\end{aligned}
$$

Table 2: Classes in $R(X)$ with respect to a type 1 basis and up to permutation of $\left(e_{i}\right)_{i>0}$. See Proposition 1.

$$
\begin{array}{cl} 
\pm\left(e_{1}-e_{2}\right), \quad & \pm\left(e_{0}-e_{1}-e_{2}-e_{3}\right), \quad \pm\left(2 e_{0}-e_{1}-e_{2}-e_{3}-e_{4}-e_{5}-e_{6}\right), \\
& \pm\left(3 e_{0}-2 e_{1}-e_{2}-e_{3}-e_{4}-e_{5}-e_{6}-e_{7}-e_{8}\right) .
\end{array}
$$

Table 3: Classes in $E(X)$ with respect to a type 1 basis and up to permutation of $\left(e_{i}\right)_{i>0}$. See Proposition 1.

$$
\begin{aligned}
& e_{1} \\
& e_{0}-e_{1}-e_{2} \\
& 2 e_{0}-e_{1}-e_{2}-e_{3}-e_{4}-e_{5} \\
& 3 e_{0}-2 e_{1}-e_{2}-e_{3}-e_{4}-e_{5}-e_{6}-e_{7}, \\
& 4 e_{0}-2 e_{1}-2 e_{2}-2 e_{3}-e_{4}-e_{5}-e_{6}-e_{7}-e_{8}, \\
& 5 e_{0}-2 e_{1}-2 e_{2}-2 e_{3}-2 e_{4}-2 e_{5}-2 e_{6}-e_{7}-e_{8}, \\
& 6 e_{0}-3 e_{1}-2 e_{2}-2 e_{3}-2 e_{4}-2 e_{5}-2 e_{6}-2 e_{7}-2 e_{8} .
\end{aligned}
$$

Table 4: Classes in $F(X)$ with respect to a type 1 basis and up to permutation of $\left(e_{i}\right)_{i>0}$. See Proposition 1.

$$
\begin{aligned}
& e_{0}-e_{1}, \\
& 2 e_{0}-e_{1}-e_{2}-e_{3}-e_{4}, \\
& 3 e_{0}-2 e_{1}-e_{2}-e_{3}-e_{4}-e_{5}-e_{6}, \\
& 4 e_{0}-2 e_{1}-2 e_{2}-2 e_{3}-e_{4}-e_{5}-e_{6}-e_{7}, \\
& 5 e_{0}-2 e_{1}-2 e_{2}-2 e_{3}-2 e_{4}-2 e_{5}-2 e_{6}-e_{7}, \\
& 4 e_{0}-3 e_{1}-e_{2}-e_{3}-e_{4}-e_{5}-e_{6}-e_{7}-e_{8}, \\
& 5 e_{0}-3 e_{1}-2 e_{2}-2 e_{3}-2 e_{4}-e_{5}-e_{6}-e_{7}-e_{8}, \\
& 6 e_{0}-3 e_{1}-3 e_{2}-2 e_{3}-2 e_{4}-2 e_{5}-2 e_{6}-e_{7}-e_{8}, \\
& 7 e_{0}-3 e_{1}-3 e_{2}-3 e_{3}-3 e_{4}-2 e_{5}-2 e_{6}-2 e_{7}-e_{8}, \\
& 7 e_{0}-4 e_{1}-3 e_{2}-2 e_{3}-2 e_{4}-2 e_{5}-2 e_{6}-2 e_{7}-2 e_{8}, \\
& 8 e_{0}-3 e_{1}-3 e_{2}-3 e_{3}-3 e_{4}-3 e_{5}-3 e_{6}-3 e_{7}-e_{8}, \\
& 8 e_{0}-4 e_{1}-3 e_{2}-3 e_{3}-3 e_{4}-3 e_{5}-2 e_{6}-2 e_{7}-2 e_{8}, \\
& 9 e_{0}-4 e_{1}-4 e_{2}-3 e_{3}-3 e_{4}-3 e_{5}-3 e_{6}-3 e_{7}-2 e_{8}, \\
& 10 e_{0}-4 e_{1}-4 e_{2}-4 e_{3}-4 e_{4}-3 e_{5}-3 e_{6}-3 e_{7}-3 e_{8}, \\
& 11 e_{0}-4 e_{1}-4 e_{2}-4 e_{3}-4 e_{4}-4 e_{5}-4 e_{6}-4 e_{7}-3 e_{8} .
\end{aligned}
$$


Proof. Assertion a) follows from [6, Theorem 8.1.15].

b) Recall Notation 1. If $q=\left(c \cdot e_{0}\right) k+3 c$, then it follows from a) that $q \cdot e_{0}=0$ and thus $q^{2}<0$ by $(\mathrm{HI})$ so that $\left(c \cdot e_{0}\right)(-k \cdot c)>\frac{9}{6} c^{2}$. If $c \cdot e_{0}>-3$, then $(k-c) \cdot e_{0}<0$ and thus $h^{2}(c)=0$ by (SD) and $e_{0}$ being nef. For each of the choices for $\left(-k \cdot c, c^{2}\right)$ we have that $c \cdot e_{0}>-3$ and $c^{2}-k \cdot c \geq 0$. It follows that $h^{0}(c)>0$ by $(\mathrm{RR})$.

c) For a given class $d=d_{0} e_{0}+\ldots+d_{r} e_{r}$ in $N(X)$ and $\alpha, \beta \in \mathbb{Z}$ we describe a procedure that computes $\left\{c \in N(X) \mid d \cdot c=\alpha, c^{2}=\beta, c \cdot e_{i}>0\right.$ for $\left.0 \leq i \leq r\right\}$. We use the notation $c=c_{0} e_{0}+\ldots+c_{r} e_{r}$ for $c \in N(X)$. It follows from the Cauchy-Schwarz inequality that

$$
\left(d_{0} c_{0}-\alpha\right)^{2}=\left(\sum_{i>0} d_{i} c_{i}\right)^{2} \leq\left(\sum_{i>0} d_{i}^{2}\right)\left(\sum_{i>0} c_{i}^{2}\right)=\left(d_{0}^{2}-d^{2}\right)\left(c_{0}^{2}-\beta\right) .
$$

Thus we require that $f\left(c_{0}\right) \leq 0$ where $f(t):=\left(d_{0} t-\alpha\right)^{2}-\left(d_{0}^{2}-d^{2}\right)\left(t^{2}-\beta\right)$.

We start the procedure with $c_{0}:=1$. We compute all $\left(c_{i}\right)_{i>0} \in \mathbb{Z}_{\geq 0}^{r}$ such that $d_{0} c_{0}-\alpha=\sum_{i>0} d_{i} c_{i}$ by going through integer partitions of $d_{0} c_{0}-\alpha$. If $\left(d \cdot c, c^{2}\right)=$ $(\alpha, \beta)$, then we add $c$ to our list. We increase $c_{0}$ with one and repeat the above steps. Notice that if $f\left(c_{0}\right)>f\left(c_{0}-1\right)$ and $f\left(c_{0}\right)>0$, then $f(t)>0$ for all $t \geq c_{0}$. Thus in this case we stop as we obtained all possible elements of the required set.

It follows from a) and Definition 2 that if $h^{0}(c)>0$ for $c \in N(X)$, then either $c \cdot e_{i}>0$ for $0 \leq i \leq r$ or $c \in\left\{e_{i}, e_{i}-e_{j} \mid 1 \leq i, j \leq r\right\}$. Assertion c) now follows from applying b) and computing the sets $R(X), E(X), F(X)$ and $\{c \in$ $\left.N(X) \mid-k \cdot c=c^{2}=1\right\}$ using the above procedure. We verify that the latter set is equal to $\{-k\}$ as asserted. For $R(X)$ and $E(X)$ see alternatively [6, Proposition 8.2.7 and Proposition 8.2.19].

Lemma 1. Suppose that $X$ is a weak del Pezzo surface with canonical class $k$ such that $1 \leq k^{2} \leq 7$. If $c \in E(X) \cup F(X)$, then $c=u+b$ such that $b:=\sum_{i \in I} b_{i}$ with $b_{i} \in B(X)$ for all $i \in I$ and either

i) $u \in E_{\star}(X), u$ is indecomposable and $h^{0}(u)=1$,

ii) $u \in F_{\star}(X), u$ is the movable part of $c$ and $h^{0}(u)=2$, or

iii) $k^{2} \leq 2$ and $u, c \notin E_{\star}(X) \cup F_{\star}(X)$.

Moreover, if $c \in E_{\star}(X) \cup F_{\star}(X)$, then $I=\emptyset$. 
Proof. We use Notation 1. Suppose that $v \in N(X)$ such that $h^{0}(v)>0$ and let $v=v_{1}+\ldots+v_{t}$ a decomposition into a sum of $t \geq 1$ indecomposable classes such that $-k \cdot v_{1} \geq-k \cdot v_{i}$ for all $i$.

Claim 1: If $h^{0}(v)=1$ and $0 \leq-k \cdot v \leq 1$, then $v_{i} \in E_{\star}(X) \cup B(X)$ for all $1 \leq i \leq t$. Since $-k$ is nef, we find that $0 \leq-k \cdot v_{1} \leq 1$ and $-k \cdot v_{i}=0$ for all $i>1$. It follows from (HI) and $k^{2}>0$ that $v_{i}^{2} \leq 0$ and thus $v_{i} \in B(X)$ for $i>1$ by (AF). Suppose that $-k \cdot v_{1}=1$ so that $h^{2}\left(v_{1}\right)=0$ by (SD) and thus $v_{1}^{2}<0$ by (RR) so that $v_{1}^{2}=-1$ by $(\mathrm{AF})$. Since $v_{1} \cdot v_{i} \geq 0$ for all $i>1$ it follows that $v_{1} \in E_{\star}(X)$. If $-k \cdot v_{1}=0$, then $v_{1} \in B(X)$ and thus claim 1 holds.

Claim 2: If $-k \cdot v=1$, then either $v^{2}=-1$, or $v=-k$ with $k^{2}=1$.

By Remark 2 we may assume without loss of generality that $h=-3 k$ so that $v$ is the class of a complex irreducible cubic in $X$. Thus $0 \leq p_{a}(v) \leq 1$ by $[12$, Example IV.6.4.2] so that $v^{2}= \pm 1$ by (AF). Claim 2 now follows from Proposition 1c.

Since $c \in E(X) \cup F(X)$, it follows from Proposition $1 \mathrm{~b}$ that $h^{0}(c)>0$. Let $c=m+f$, where $m$ and $f$ are the movable and fixed part of $c$, respectively.

Suppose that $c \in E(X)$. If $m=0$, then (i) holds by claim 1 . If $m \neq 0$, then we know from (HI), $k^{2}>0$ and $m^{2} \geq 0$ that $k \cdot m \neq 0$. Therefore, $-k \cdot m=1$ as $-k$ is nef. It follows from claim 2 that $m^{2}=1$ and $m=-k$ so that (iii) holds.

Suppose that $c \in F(X)$. Notice that $m \neq 0$, since otherwise $h^{0}(f) \geq 2$ by (SD) and (RR). Since $-k \cdot m-k \cdot f=2$ and $-k$ is nef, we find that $1 \leq-k \cdot m \leq 2$.

Suppose that $-k \cdot m=1$. It follows from claim 1 with $v=f$ and claim 2 with $v=m$ that $c=-k+e+b$ such that $e \in E_{*}(X)$ and $k^{2}=1$. Thus $u=-k+e$ so that $c \cdot u=2+b \cdot e>0$. Since $c^{2}=c \cdot u+c \cdot b=0$ it follows that $c \cdot b<0$ and thus (iii) holds as $c \cdot b_{i}<0$ for some $i \in I$.

Suppose that $-k \cdot m=2$ so that $-k \cdot f=0$. It follows from claim 1 that $u=m$ and $b=f$. Recall from Remark 2 that if $k^{2} \geq 3$, then without loss of generality $h=-k$ so that $m$ is the class of conic and thus $u^{2}=0$ by (AF). By (RR) and (KV) we find that $h^{0}(u)=\frac{1}{2} u^{2}+2$. Thus if $k^{2} \geq 3$ or $u^{2}=0$, then (ii) holds. Suppose that $k^{2} \leq 2$ and $u^{2}>0$. It follows from (HI) and $c^{2}=0$ that $u \cdot c>0$. Since $c^{2}=u \cdot c+b \cdot c=0$ we find that $c \cdot b<0$ so that (iii) holds as $c \cdot b_{i}<0$ for some $i \in I$. 
Suppose by contradiction that $c \in E_{\star}(X) \cup F_{\star}(X)$ and $I \neq \emptyset$. We are in (i) or (ii) so that $c=u+b$ and $u \in E_{\star}(X) \cup F_{\star}(X)$. It follows from $k^{2}>0$ and (HI) that $b^{2}<0$. Since $c^{2}=u^{2}+b \cdot u+c \cdot b=u^{2}$ we find that $c \cdot b=-u \cdot b$. We have $c \cdot b=u \cdot b+b^{2}$ and it follows from $k^{2}>0$ and (HI) that $b^{2}<0$. We arrived at a contradiction, since $c \cdot b<0$ and thus $c \cdot b_{i}<0$ for some $i \in I$.

Lemma 2. Suppose that $Y \rightarrow X$ is the smooth model of a weak del Pezzo surface $X$ with canonical class $k$. If $e \in E_{\star}(Y)$, then $e$ is the class of a complex $(-1)$-curve that can be contracted such that the resulting smooth complex surface $Y^{\prime}$ is a complex weak del Pezzo surface of canonical degree $2 \leq k^{2}+1 \leq 9$ and $N\left(Y^{\prime}\right)$ is isomorphic to the sublattice $\{c \in N(Y) \mid c \cdot e=0\}$. If $k^{2}=9$, then $Y \cong \mathbb{P}^{2}$ and $N(Y)=\left\langle e_{0}\right\rangle_{\mathbb{Z}}$. If $k^{2}<8$, then $Y$ is complex isomorphic to the blowup of $\mathbb{P}^{2}$ in $9-k^{2}$ points.

Proof. See [6, Proposition 8.1.23 and Theorem 8.1.15].

The following lemma will be used only later in Lemma 13, but now is the right time to prove it.

Lemma 3. Suppose that $X$ is a weak del Pezzo surface with canonical class $k$.

a) If $k^{2} \leq 7$ and $f \in F_{\star}(X)$, then there exists $e \in E_{\star}(X)$ such that $f \cdot e=0$.

b) If $k^{2} \leq 7$ and $f_{1}, f_{2} \in F_{\star}(X)$ such that $f_{1} \cdot f_{2}=1$, then there exists $e \in E_{\star}(X)$ such that $f_{1} \cdot e=f_{2} \cdot e=0$.

c) If $k^{2} \leq 5$ and $f_{1}, f_{2}, f_{3} \in F_{\star}(X)$ such that $f_{1} \cdot f_{2}=f_{1} \cdot f_{3}=f_{2} \cdot f_{3}=1$, then there exists $e \in E_{\star}(X)$ such that $f_{1} \cdot e=f_{2} \cdot e=f_{3} \cdot e=0$.

Proof. Suppose that $g \in F_{\star}(X)$ and that there exists $e \in E(X)$ such that $g \cdot e=0$. By Lemma 1 we find that $e=u+b$ where $u \in E_{\star}(X)$ and $b$ is a sum of classes in $B(X)$. We have $g \cdot b \geq 0$ by assumption and $g \cdot u \geq 0$ by Lemma 1 . It follows that there exists $u \in E_{\star}(X)$ such that $g \cdot u=0$. Therefore, we may assume without loss of generality that $B(X)=\emptyset$ in the proofs of the assertions a), b) and c). In other words, we assume without loss of generality that $E_{\star}(X)=E(X)$.

Suppose that $c \in E(X) \cup F(X)$. By Proposition 1, $c$ is, up to permutation of the $\left(e_{j}\right)_{j}$, defined by the $\lambda$-th row in Table 3 or Table 4 . We use in this case the notation $c \rightarrow \lambda$. For example, $e_{1} \rightarrow 1, e_{8} \rightarrow 1, e_{0}-e_{2} \rightarrow 1$ and $2 e_{0}-e_{4}-e_{5}-e_{6}-e_{7} \rightarrow 2$. 
a) First we suppose that $k^{2}=1$. Suppose by contradiction that there does not exist $e \in E(X)$ such that $e \cdot f=0$. Notice that $f \rightarrow \lambda$ for some row $1 \leq \lambda \leq 15$. We find that $\lambda \geq 4$, otherwise $e \cdot f=0$ for some $e \in E(X)$ such that $e \rightarrow 1$. Moreover, $\lambda \in\{9,11,12,13,14,15\}$, otherwise $e \cdot f=0$ for some $e \in E(X)$ such that $e \rightarrow 2$. Next, we observe that $\lambda \in\{11,13,14,15\}$, otherwise $e \cdot f=0$ for some $e \in E(X)$ such that $e \rightarrow 3$. By the same argument we have $r \in\{15\}$, otherwise $e \cdot f=0$ for some $e \in E(X)$ such that $e \rightarrow 4$ or $e \rightarrow 5$. We arrived at a contradiction as $e \cdot f=0$ for some $e \in E(X)$ such that $e \rightarrow 6$. The cases for $2 \leq k^{2} \leq 7$ can be shown analogously.

b) It follows from a) that $f_{1} \cdot e=0$ for some $e \in E(X)$. If $f_{2} \cdot e \neq 0$, then $f_{2} \cdot e>0$ by Lemma 1. We find that $f_{1}-e$ is in $E(X)$ and orthogonal to $f_{1}$. Again by Lemma 1 we have that $f_{2} \cdot\left(f_{1}-e\right) \geq 0$ and thus $f_{2} \cdot\left(f_{1}-e\right)=0$ so that we concluded the proof.

c) We know from b) that there exists $e \in E_{\star}(X)$ such that $f_{1} \cdot e=f_{2} \cdot e=0$. We subsequently apply Lemma 2 and b) so that we obtain a complex birational morphism $\varphi: X \rightarrow X^{\prime}$ where $X^{\prime}$ is a weak del Pezzo surface of canonical degree 7 and $\varphi_{*} f_{1}, \varphi_{*} f_{2} \in F(X)$. It follows from Proposition 1c that we may assume without loss of generality that $\varphi_{*} f_{1}=e_{0}-e_{1}$ and $\varphi_{*} f_{2}=e_{0}-e_{2}$. Thus it follows from Lemma 2 that $N(X) \cong\left\langle e_{0}, \ldots, e_{r}\right\rangle_{\mathbb{Z}}$ with $r=9-k^{2} \geq 4$ such that $f_{1}=e_{0}-e_{1}$, $f_{2}=e_{0}-e_{2}$ and $e_{3}, \ldots, e_{r} \in E_{\star}(X)$ are the classes of the complex $(-1)$-curves that are contracted by $\varphi$. It is now a straightforward consequence of Proposition $1 \mathrm{c}$ and $f_{1} \cdot f_{3}=f_{2} \cdot f_{3}=1$ that either $f_{3} \rightarrow 1$ or $f_{3} \rightarrow 2$. In both cases there exists $u \in E(X)$ with either $u \rightarrow 1$ or $u \rightarrow 2$ such that $f_{1} \cdot u=f_{2} \cdot u=f_{3} \cdot u=0$ and thus we concluded the proof.

Proposition 2. Suppose that $X \subset \mathbb{P}^{n}$ is a weak del Pezzo surface with canonical class $k$ such that $1 \leq k^{2} \leq 6$. Let $h$ denote the class of hyperplane sections of $X$, let $Y \rightarrow X$ be the smooth model and let $X_{N}$ be a linear normalization of $X$.

a) We have that $W \subset N(X)$ is the set of classes of all complex irreducible curves in $Y$ that contract to some complex isolated singularity of $X_{N}$ if and only if $W$ is a component of $B(X)$. If $\sigma_{*}(W)=W$, then the corresponding isolated singularity is real. 
b) We have that $c \in E_{\star}(X)$ if and only if $c=[C]$ and $C \subset X$ is an unmovable complex minimal degree rational curve. If $c \in E_{\mathbb{R}}(X)$, then the corresponding curve is real. If $h=-k$, then the unmovable rational curves on $X$ are lines.

c) We have that $c \in F_{\star}(X)$ if and only if $c$ is the class of a 1-dimensional complex minimal family of $X$. If $c \in F_{\mathbb{R}}(X)$, then the corresponding family is simple. If $h=-k$, then simple curves on $X$ are conics.

Proof. The assertion a) is a direct consequence of [6, Proposition 8.1.9, Proposition 8.1.10 and Theorem 8.2.27]. Assertions b) and c) are straightforward consequences of Lemma 1.

Notation 2. Let $\mathbf{F}_{0}$ and $\mathbf{F}_{0}^{\prime}$ be both isomorphic to $\mathbb{P}^{1} \times \mathbb{P}^{1}$, but with $\sigma_{*}$ acting trivial and non-trivial, respectively. Let $\mathbf{F}_{1}$ denote the blowup of $\mathbb{P}^{2}$ in one point. Let $\mathbf{F}_{2}$ denote the blowup of $\mathbb{P}^{2}$ in two infinitely near points followed by the contraction of the pullback of the line through these points. Here $\sigma_{*}: N\left(\mathbf{F}_{i}\right) \rightarrow N\left(\mathbf{F}_{i}\right)$ acts trivially for $1 \leq i \leq 2$. Let $\mathbf{G}_{2}$ denote the blowup of $\mathbb{P}^{2}$ in two complex conjugate points so that $N\left(\mathbf{G}_{2}\right)=\left\langle e_{0}, e_{1}, e_{2}\right\rangle_{\mathbb{Z}}$ is a type 1 basis.

Lemma 4. Suppose that $Y \rightarrow X$ is the smooth model of a weak del Pezzo surface $X \subset \mathbb{P}^{n}$ with canonical class $k$. Either one of the following 8 cases holds (we use Notation 2 and Table 1):

a. $k^{2}=9, Y \cong \mathbb{P}^{2}$ and $G(X)=\left\{-\frac{1}{3} k\right\}$.

b. $k^{2}=8, Y \cong \mathbf{F}_{0}$ and $G(X)=F_{\mathbb{R}}(X)$.

c. $k^{2}=8, Y \cong \mathbf{F}_{0}^{\prime}$ and $G(X)=\left\{-\frac{1}{2} k\right\}$.

d. $k^{2}=8, Y \cong \mathbf{F}_{1}$ and $G(X)=F_{\mathbb{R}}(X)$.

e. $k^{2}=8, Y \cong \mathbf{F}_{2}$ and $G(X)=F_{\mathbb{R}}(X)$.

f. $k^{2}=7, Y \cong \mathbf{G}_{2}$ and $G(X)=\left\{e_{0}\right\}$.

g. $1 \leq k^{2} \leq 7$ and $G(X)=F_{\mathbb{R}}(X) \neq \emptyset$.

h. $1 \leq k^{2} \leq 2, G(X)=\emptyset$ and $X$ is not $\mathbb{R}$-rational.

Proof. For $9 \geq k^{2} \geq 7$, the assertion is a straightforward consequence of $[6$, Section 8.4.1].

Now, suppose that $X$ is $\mathbb{R}$-rational and $1 \leq k^{2} \leq 6$. Thus $Y$ is the blowup of either $\mathbb{P}^{2}$ in at least three complex points, or the blowup of $\mathbb{P}^{1} \times \mathbb{P}^{1}$ in at least two complex 
points. It follows from Proposition 1c and Lemma 1 that if $Y$ is the blowup of $\mathbb{P}^{2}$ in at least one real point, then without loss of generality $e_{0}-e_{1} \in F_{\mathbb{R}}(X)$. Similarly, if $Y$ is the blowup of $\mathbb{P}^{2}$ in four complex conjugate points, then without loss of generality $2 e_{0}-e_{1}-e_{2}-e_{3}-e_{4} \in F_{\mathbb{R}}(X)$. If $Y$ is the blowup of $\mathbb{P}^{1} \times \mathbb{P}^{1}$ in two complex conjugate points, then the pullback of a curve of bidegree $(1,1)$, that passes through these points, has class $c \in F(X)$ such that $\sigma_{*}(c)=c$ so that $F_{\mathbb{R}}(X) \neq \emptyset$ by Lemma 1. Thus if $X$ is $\mathbb{R}$-rational, then $G(X)=F_{\mathbb{R}}(X)$ by Proposition 2c.

We established that if $F_{\mathbb{R}}(X)=\emptyset$ and $k^{2} \leq 6$, then $X$ is not $\mathbb{R}$-rational and thus $X$ is not covered by a complete rational family (see Definition 3 ) so that $G(X)=\emptyset$. It follows from [30, Theorem 4.6] that $1 \leq k^{2} \leq 2$.

Remark 3. Suppose that $X \subset \mathbb{P}^{n}$ is an algebraic surface. We recover the simple family graph $\mathcal{G}(X)$ uniquely from $G(X)$, by removing from the incidence diagram $\mathfrak{D}(G(X))$ the self-loops and the edges labeled 1 , and by labeling each vertex $c \in$ $G(X)$ with $h^{0}(c)-1$.

\section{Cremona invariant for weak del Pezzo surfaces}

We will introduce an invariant for Neron-Severi lattices of weak del Pezzo surfaces.

We start by recalling some concepts of root systems and we mainly follow [11, Chapter 8]. Let $(V, \cdot)$ be a real inner product space. A root system is a finite subset $R \subset V$ with the following properties:

1. The set $R$ spans $V$.

2. If $r \in R$ and $\alpha r \in R$ for $\alpha \in \mathbb{R}$, then $\alpha \in\{1,-1\}$.

3. The set $R$ is closed under reflection through the hyperplane perpendicular to any $r \in R: s-2(s \cdot r / r \cdot r) r \in R$ for all $r, s \in R$.

4. The projection of $s \in R$ onto the line through $r \in R$ is a half-integral multiple of $r: 2(s \cdot r / r \cdot r) \in \mathbb{Z}$.

We call $B \subset R$ a root base of a root system $R$ if $B$ is a basis for $V$ and if every element in $R$ can be expressed as an integral linear combination of elements in $B$ with all coefficients either all positive or all negative. 
The Dynkin diagram of a root system $R$ is defined as the incidence diagram of a root base $B \subset R$ (see Definition 5) and is independent of the choice of root base. In this article, the label of an edge in a Dynkin diagram is 1 if the edge is not a self-loop and all vertices in our Dynkin diagrams admit a self-loop labeled -2 . Thus we can ignore the labelings and self-loops so that the Dynkin diagrams in this text will be isomorphic to either the empty graph $A_{0}$ or one of the Dynkin graphs $A_{\ell}, D_{\ell}$ or $E_{\ell}$ with $\ell$ vertices.

Root systems $R$ and $R^{\prime}$ are isomorphic if and only if there exists an isomorphism $\varphi:(V, \cdot) \rightarrow(V, \cdot)$ such that $\varphi(R)=R^{\prime}$. In [11, Definition 8.5] the notion of isomorphism is slightly weaker, but equivalent in the setting of this paper. The Dynkin diagram of a root system determines uniquely its isomorphism class.

A root subsystem is a subset $S \subset R$ that forms root system in the vector space spanned by $S$. Two root subsystems $(S, R)$ and $\left(S^{\prime}, R^{\prime}\right)$ are isomorphic if and only if there exists an isomorphism of root systems $\varphi: R \rightarrow R^{\prime}$ such that $\varphi(S)=S^{\prime}$. The Dynkin diagram of a root subsystem does in general not uniquely determine its isomorphism class.

Let us consider the distinguished subsets of $N(X)$ as stated in Table 1 . We consider the following inner product space

$$
V_{k}(X):=\left(\{c \in N(X) \mid k \cdot c=0\} \otimes_{\mathbb{Z}} \mathbb{R}, \cdot\right) .
$$

The following proposition summarizes some known facts from the literature.

Proposition 3. Let $X$ and $X^{\prime}$ be weak del Pezzo surfaces such that $1 \leq k^{2}=k^{\prime 2} \leq 8$, $h=-\alpha k$ and $h^{\prime}=-\alpha k^{\prime}$ for some fixed $\alpha \in \mathbb{Q}_{>0}$.

a) $R(X)$ forms a root system in the inner product space $V_{k}(X)$ such that

\begin{tabular}{r|cccccccc}
$k^{2}$ & 8 & 7 & 6 & 5 & 4 & 3 & 2 & 1 \\
Dynkin diagram of $R(X)$ & $A_{1}$ & $A_{1}$ & $A_{1}+A_{2}$ & $A_{4}$ & $D_{5}$ & $E_{6}$ & $E_{7}$ & $E_{8}$
\end{tabular}

b) Subsets $S(X)$ and $T(X)$ form root subsystems of $R(X)$ with root bases $A(X)$ and $B(X)$, respectively.

c) Suppose that $\sigma_{*}$ is the identity. One has $\mathbf{N}(X) \cong \mathbf{N}\left(X^{\prime}\right)$ if and only if $T(X) \cong$ $T\left(X^{\prime}\right)$ as root subsystems.

d) Suppose that $B(X)$ is the emptyset. One has $\mathbf{N}(X) \cong \mathbf{N}\left(X^{\prime}\right)$ if and only if $S(X) \cong S\left(X^{\prime}\right)$ as root subsystems. 
Proof. Assertions a) and b) follow from [6, Proposition 8.2.10 and Proposition 8.2.25] and [33, Section 2] (see also Remark 4). Notice that if $\sigma_{*}$ is the identity or if $B(X)=\emptyset$, then we can consider $X$ as a complex weak del Pezzo surface and a (nonweak) del Pezzo surface, respectively. Assertion c) is now a direct consequence of [6, Corollary 8.2.33]. Assertion d) is a direct consequence of [33, Theorem 2.1].

Remark 4. The matrix defining $\sigma_{*}: N(X) \rightarrow N(X)$ has eigenvalues \pm 1 , since it is an involution. Let $V^{ \pm}$denote the eigenspace of $\sigma_{*}$ for eigenvalues \pm 1 , respectively. Notice that $V^{-} \subset V_{k}(X)$, since $\sigma_{*}(k)=k$ and $\sigma_{*}(v) \cdot \sigma_{*}(k)=-v \cdot k$ for all $v \in V^{-}$. The intersection of a root system with a subspace is a root subsystem and $V^{-} \cap R(X)$ generates $V^{-}$. Thus $S(X)$ is a root subsystem of $R(X)$ and $A(X)$ is its root base by definition.

Lemma 5. Let $X$ be a weak del Pezzo surface. The function $h^{0}: N(X) \rightarrow \mathbb{Z}_{\geq 0}$ is uniquely determined by $B(X)$. Thus the Neron-Severi lattice $\mathbf{N}(X)$ is uniquely determined by $B(X)$ together with data 1, 2 and 3 in Definition 1.

Proof. Recall Notation 1, see Table 1 for $E(X), B(X) \subset N(X)$ and let $k$ denote the canonical class of $X$ such that $-k$ is nef and big by Definition 4. By Proposition 1 we may assume that $\left\langle e_{0}, e_{1}, \ldots, e_{r}\right\rangle_{\mathbb{Z}}$ is a type 1 basis for $N(X)$ such that $r=9-k^{2}$, $k=-3 e_{0}+e_{1}+\ldots+e_{r}$ and the elements of $E(X)$ are characterized by Table 3 . We assume that we have explicit coordinates for the elements in $B(X)$ in terms of this type 1 basis and we recover $E_{\star}(X)$ from $E(X)$ and $B(X)$ by applying Lemma 1 .

Claim 1: If $c \in N(X)$ such that $h^{0}(c)>0$ and $c \cdot q<0$ for some indecomposable class $q$, then $q \in E_{\star}(X) \cup B(X)$ and $h^{0}(c)=h^{0}(c-q)$.

We know from $(\mathrm{RR})$ and $(\mathrm{SD})$ that $h^{0}(q)=1 \geq \frac{1}{2}\left(q^{2}-k \cdot q\right)+1$ so that $-q^{2}+k \cdot q \geq 0$. Therefore, by (AF) and $-k$ being nef, we find that $0 \leq-k \cdot q \leq 1$ and $q^{2} \leq 0$. If $-k \cdot q=0$, then $q^{2}<0$ by (HI) and thus $q^{2}=-2$ by $(\mathrm{AF})$. If $-k \cdot q=1$, then $q^{2}=-1$ by $(\mathrm{AF})$. It follows from Lemma 1 that $q \in E_{\star}(X) \cup B(X)$. Since $q$ is the class of a fixed component of the linear series of $c$ we find that $h^{0}(c)=h^{0}(c-q)$. Hence, we concluded the proof of claim 1.

For given $c \in\left\langle e_{0}, e_{1}, \ldots, e_{r}\right\rangle_{\mathbb{Z}}$ we would like to recover $h^{0}(c)$ from $E_{\star}(X) \cup B(X)$. If $c \cdot q<0$ for some $q \in E_{\star}(X) \cup B(X)$, then we apply claim 1 and set $c:=c-q$. We repeat this step until either $c \cdot q \geq 0$ for all $q \in E_{\star}(X) \cup B(X)$ or $c \cdot e_{0}<0$. If $c \cdot e_{0}<0$, then $h^{0}(c)=0$. Otherwise $h^{0}(c)=\frac{1}{2}\left(c^{2}-k \cdot c\right)+1$ by $(\mathrm{RR})$ and $(\mathrm{KV})$. 
The pair of Dynkin diagrams $\mathfrak{D}(A(X))$ and $\mathfrak{D}(B(X))$ is not fine enough as an invariant for the Neron-Severi lattice $\mathbf{N}(X)$ of a weak del Pezzo surface $X$, since nonisomorphic Neron-Severi lattices might have the same such pair. For this purpose we introduce a more fine-grained and computable version of the Dynkin diagram. The Cremona invariant is defined as

$$
\mathfrak{C}(X):=\mathfrak{D}(E(X) \cup B(X)) \bigcup\left\{\left(c, \sigma_{*}(c), \infty\right) \mid c \in E(X) \cup B(X)\right\}
$$

Thus we enhance the incidence diagram of $E(X) \cup B(X)$ with edges (labeled $\infty$ ) between conjugate classes. Notice that $\sigma_{*}(B(X))=B(X)$. We consider Cremona invariants isomorphic if they are isomorphic as labeled graphs.

Proposition 4. Suppose that $X$ and $X^{\prime}$ are weak del Pezzo surfaces such that $1 \leq k^{2}, k^{\prime 2} \leq 7, h=-\alpha k$ and $h^{\prime}=-\alpha k^{\prime}$ for some fixed $\alpha \in \mathbb{Q}_{>0}$. One has $\mathbf{N}(X) \cong \mathbf{N}\left(X^{\prime}\right)$ if and only if $\mathfrak{C}(X) \cong \mathfrak{C}\left(X^{\prime}\right)$.

Proof. The $\Rightarrow$ direction is immediate. In the remainder of this proof we consider the $\Leftarrow$ direction. It is a straightforward consequence of Proposition 1[a,c] that $N(X) \cong\left\langle e_{0}-e_{1}-e_{2}, e_{1}, \ldots, e_{r}\right\rangle_{\mathbb{Z}}$, where the generators are a subset of $E(X)$. The graph isomorphism restricted to these generators, uniquely induces an isomorphism $\lambda: N(X) \rightarrow N\left(X^{\prime}\right)$ of $\mathbb{Z}$-modules. It is left to verify that this isomorphism is compatible with the remaining data of $\mathbf{N}(X)$ as it is stated in Definition 1 . The edges labeled $\infty$ uniquely determines the involution $\sigma_{*}$ on the generators such that $\lambda \circ \sigma_{*}=\sigma_{*} \circ \lambda$. The canonical class $k$ of $X$ is the unique element in $N(X)$ such that $k^{2}=10-\operatorname{rank}(N(X)), \sigma_{*}(k)=k$ and $k \cdot e=-1$ for all $e \in E(X)$. It follows that $\lambda(k)=k^{\prime}$. It follows from Lemma 5 and $\lambda(B(X))=B\left(X^{\prime}\right)$ that $h^{0}=h^{0} \circ \lambda$. Thus we constructed an isomorphism of Neron-Severi lattices and concluded the proof.

\section{A classification of weak del Pezzo surfaces}

In this section we prove Theorem 2 via an algorithm that outputs Table 7 . The algorithm is designed so that it is easy to implement in a computer algebra system, while still terminating in less than two days on a single pentium processor. The computation of Table 7 for $d \geq 3$ takes only a couple of minutes. See [16, ns_lattice] for an implementation. 
The set of positive roots $R^{+}$is defined as the set of classes $c \in\left\langle e_{0}, \ldots, e_{8}\right\rangle_{\mathbb{Z}}$ that are listed up to permutation of $\left(e_{i}\right)_{i}$ in Table 2 such that either $c=e_{i}-e_{j}$ for some $1 \leq i<j \leq 8$ or $c \cdot e_{0}>0$.

Lemma 6. Suppose that $X \subset \mathbb{P}^{n}$ is a weak del Pezzo surface such that $1 \leq k^{2} \leq 7$. There exists a type 1 basis for $N(X)$ such that $A(X) \subset R^{+}$. Moreover, for all unimodular involutions $\sigma_{*}: N(X) \rightarrow N(X)$, such that $A(X) \subset R^{+}$is a root base for $S(X)$, we may assume without loss of generality that $B(X) \subset R^{+}$.

Proof. Let $Y \rightarrow X$ be the smooth model and let $X_{N}$ be a linear normalization. By Proposition 1a, there exists a sequential blowup $\pi: Y \rightarrow \mathbb{P}^{2}$ with center $\left(p_{1}, \ldots, p_{r}\right)$ so that $N(X)$ admits a type 1 basis $\left\langle e_{0}, \ldots, e_{r}\right\rangle_{\mathbb{Z}}$. We follow the notation of Definition 2 and let $C_{1} \subset Y_{1}=\mathbb{P}^{2}$ be a curve. The Zariski closure $C_{2} \subset Y_{2}$ of $\pi_{1}^{-1}\left(C_{1} \backslash\left\{p_{1}\right\}\right)$ is called the strict transform of $C_{1}$. The strict transform of $C_{1}$ via $\pi_{1} \circ \pi_{2}$ is defined as the strict transform of $C_{2}$ and so on. Recall from Remark 4 that the root base $A(X)$ forms a basis of the eigenspace of -1 for the involution $\sigma_{*}$. Since positive roots in $A(X)$ are send by $\sigma_{*}$ to negative roots, we may assume without loss of generality that $A(X) \subset R^{+}$. Any other choice for $\sigma_{*}$, such that $A(X) \subset R^{+}$is a root base for $S(X)$, is equivalent to a choice for a type 1 basis for $N(X)$. If $X_{N} \cong Y$, then $B(X)=\emptyset$ by Proposition 2a and the complex blowup centers $p_{1}, \ldots, p_{r}$ are general. Now suppose that $X_{N} ¥ Y$. In this case $p_{1}, \ldots, p_{r}$ lie in "almost" general position in the following sense. If $e_{0}-e_{1}-e_{2}-e_{3} \in B(X)$, then $p_{1}, p_{2}$ and $p_{3}$ lie on strict transforms of a complex line. If $2 e_{0}-e_{1}-e_{2}-e_{3}-e_{4}-e_{5}-e_{6} \in B(X)$, then $p_{1}, \ldots p_{6}$ lie on strict transforms of a complex conic. If $3 e_{0}-2 e_{1}-e_{2}-e_{3}-e_{4}-e_{5}-e_{6}-e_{7}-e_{8} \in B(X)$, then $p_{1}, \ldots p_{8}$ lie on a strict transforms of a complex cubic that has a double point at $p_{1}$. If $e_{1}-e_{2} \in B(X)$, then $p_{2}$ is infinitely near to $p_{1}$. It follows from Proposition $1 \mathrm{c}$ that these are, up to permutation of $p_{1}, \ldots, p_{r}$ all possible geometric conditions. It is now straightforward to see that we can always choose an index on the points such that all the conditions are expressed by elements in $R^{+}$and such that $\sigma_{*}(B(X))=B(X)$. Therefore, $B(X) \subset R^{+}$without loss of generality as was left to be shown.

Notation 3. Suppose that $X$ is a weak del Pezzo surface with canonical class $k$. In the following five algorithms we will represent $N(X)$ as a type 1 basis $\left\langle e_{0}, \ldots, e_{r}\right\rangle_{\mathbb{Z}}$ with $r=9-k^{2}$ and $R^{+}$is short notation for $R^{+} \cap N(X)$. Suppose that $B \subset R^{+}$represents a root base $B(X)$ and that matrix $M \in \mathbb{Z}^{(r+1) \times(r+1)}$ represents $\sigma_{*}: N(X) \rightarrow$ 
$N(X)$. We denote by $\mathfrak{C}(B, M)$ the Cremona invariant $\mathfrak{C}(X)$. Notice that we know $E(X) \subset\left\langle e_{0}, \ldots, e_{r}\right\rangle_{\mathbb{Z}}$ from Table 3 and thus we can uniquely recover $\mathfrak{C}(X)$ from $B$ and $M$. If $M$ is the identity matrix, then we denote $\mathfrak{C}(X)$ by $\mathfrak{C}(B)$.

Algorithm 1. (is_root_base)

- Input. A subset $B \subset R^{+}$with respect to a type 1 basis $\left\langle e_{0}, \ldots, e_{r}\right\rangle_{\mathbb{Z}}$.

- Output. True if $B$ is a root base of a root subsystem and False otherwise.

- Method. Return True if $B$ is a linear independent set in $\left\langle e_{0}, \ldots, e_{r}\right\rangle_{\mathbb{R}}$, if the edges of $\mathfrak{D}(B)$ that are not self-loops are labeled 1 and if all roots in $\langle B\rangle_{\mathbb{R}} \cap R^{+}$are a positive linear combination of elements in $B$.

\section{Algorithm 2. (seek_bases)}

- Input. An integer $1 \leq r \leq 8$ and a subset $S \subset R^{+}$.

- Output. A set $\Psi \subset\{B \subset S \mid B$ is a root base and $|B| \leq r\}$ such that $\emptyset \in \Psi$ and for all root bases $B^{\prime} \subset S$ there exists $B \in \Psi$ such that $\mathfrak{C}(B) \cong \mathfrak{C}\left(B^{\prime}\right)$. Moreover, $\mathfrak{C}(B) \nsucceq \mathfrak{C}\left(B^{\prime \prime}\right)$ for all distinct $B, B^{\prime \prime} \in \Psi$. The elements of the sets in $\Psi$ are represented with respect to a type 1 basis $\left\langle e_{0}, \ldots e_{r}\right\rangle_{\mathbb{Z}}$.

- Method. Let $\mathcal{T}$ be the set of Dynkin diagrams with at most $r$ vertices and with components of types $A_{\ell}, D_{\ell}$ and/or $E_{\ell}$ (including the empty graph $A_{0}$ ). Set $\Psi:=\{\}$. We do the following procedure for all $D \in \mathcal{T}$ where $D$ has vertices $\left\{v_{1}, \ldots, v_{n}\right\}$. We loop through all $b_{1} \in S$ for vertex $v_{1}$. For each $b_{1} \in S$ we loop through all $b_{2} \in S$ such that $b_{1} \cdot b_{2}=1$ if $\left(v_{1}, v_{2}\right)$ is an edge of $D$ and $b_{1} \cdot b_{2}=0$ otherwise. We recursively continue this procedure until finally $B=\left\{b_{1}, b_{2}, \ldots, b_{n}\right\}$ such that $\mathfrak{D}(B) \cong D$ as graphs (if such $B$ exists). We add $B$ to $\Psi$ if $\mathfrak{C}(B) \neq \mathfrak{C}\left(B^{\prime}\right)$ for all $B^{\prime} \in \Psi$. We return $\Psi$ after all possible $D \in \mathcal{T}$ and $B \subset S$ are exhausted with this method.

Algorithm 3. (get_bas)

- Input. An integer $1 \leq r \leq 8$.

- Output. A set $\Psi \subset\left\{B \subset R^{+} \mid B\right.$ is a root base and $\left.|B| \leq r\right\}$ such that $\emptyset \in \Psi$ and for all root bases $B^{\prime} \subset R^{+}$with $\left|B^{\prime}\right| \leq r$ there exists $B \in \Psi$ such that $\mathfrak{C}(B) \cong \mathfrak{C}\left(B^{\prime}\right)$. Moreover, $\mathfrak{C}(B) \nsucceq \mathfrak{C}\left(B^{\prime \prime}\right)$ for all distinct $B, B^{\prime \prime} \in \Psi$. We represent all elements of sets in $\Psi$ with respect to a type 1 basis $\left\langle e_{0}, \ldots e_{r}\right\rangle_{\mathbb{Z}}$. 
- Method.

if $r=1$ then return $\{\emptyset\}$

$\Psi:=\{\}$

$\Psi_{1}:=\operatorname{get} \_\operatorname{bas}(r-1)$

$\Psi_{2}:=$ seek_bases $\left(r,\left\{c \in R^{+} \mid c \cdot e_{r} \neq 0\right\}\right)$

for $\left(B_{1}, B_{2}\right) \in \Psi_{1} \times \Psi_{2}$ do

if is_root_base $\left(B_{1} \cup B_{2}\right)$ and $\mathfrak{C}\left(B_{1} \cup B_{2}\right) \not \mathfrak{C}\left(B^{\prime}\right)$ for all $B^{\prime} \in \Psi$ then

$$
\Psi:=\Psi \cup\left\{B_{1} \cup B_{2}\right\}
$$

return $\Psi$

Algorithm 4. (get_inv)

- Input. An integer $1 \leq r \leq 8$.

- Output. A set $\Psi$ of pairs $(A, M)$, where $A \subset R^{+}$is a root base and $M \in \mathbb{Z}^{(r+1) \times(r+1)}$ is an involutory matrix such that the elements in $A$ form a basis for the eigenspace of $M$ for eigenvalue -1 . For each such pair $\left(A^{\prime}, M^{\prime}\right)$ there exists $(A, M) \in \Psi$ such that $\mathfrak{C}(\emptyset, M) \cong \mathfrak{C}\left(\emptyset, M^{\prime}\right)$. Moreover, for each distinct $(A, M),\left(A^{\prime \prime}, M^{\prime \prime}\right) \in \Psi$ we ensured that $\mathfrak{C}(\emptyset, M) \nsucceq \mathfrak{C}\left(\emptyset, M^{\prime \prime}\right)$. Both $A$ and $M$ are represented with respect to a type 1 basis $\left\langle e_{0}, \ldots, e_{r}\right\rangle_{\mathbb{Z}}$.

- Method. We set $\Psi:=\{\}, I$ is the identity matrix, $k:=-3 e_{0}+e_{1}+\ldots+e_{r}$ and $J$ is the matrix corresponding to the inner product for the type 1 basis.

for $A \in \operatorname{get} \_$bas $(r)$ do

1. We represent the elements in $A$ as rows of a matrix. The columns of matrix $K$ form a basis for the kernel of $A \cdot J$. Let $Q:=\left(A^{\top} \mid K\right)$ be the matrix whose columns are the union of the columns of $A^{\top}$ and $K$.

2. Set $M:=Q \cdot D \cdot Q^{-1}$, where $D$ is the diagonal matrix with the first $|A|$ entries -1 and the remaining entries 1 .

3. if $M \in \mathbb{Z}^{(r+1) \times(r+1)}$ and $M^{\top} \cdot J \cdot M=J$ and $M \cdot M=I$ and $M(k)=k$ and $\mathfrak{C}(\emptyset, M) \nsucceq \mathfrak{C}\left(\emptyset, M^{\prime}\right)$ for all $\left(A^{\prime}, M^{\prime}\right) \in \Psi$ then $\Psi:=\Psi \cup\{(A, M)\}$

return $\Psi$

$\triangleleft$ 
Algorithm 5. (get_cls)

- Input. An integer $1 \leq r \leq 8$.

- Output. The set $\Psi:=\{[\mathbf{N}(X)] \mid X$ is a weak del Pezzo surface, $\operatorname{rank}(N(X))=r+1, h=-k\}$, where the equivalence class $[\mathbf{N}(X)]$ is represented by a triple $(B, A, M)$ where $B, A \subset$ $R^{+}$represent $B(X), A(X) \subset R(X)$ with respect to a type 1 basis $\left\langle e_{0}, \ldots, e_{r}\right\rangle_{\mathbb{Z}}$ and $M \in \mathbb{Z}^{(r+1) \times(r+1)}$ represents $\sigma_{*}: N(X) \rightarrow N(X)$.

- Method. Let $I$ denote the $(r+1) \times(r+1)$ identity matrix.

if $r=1$ then return $\{(\emptyset, \emptyset, I)\}$

$\Psi:=\{\}$

for $(A, M) \in$ get_inv $(r)$ do

$\Psi_{1}:=\left\{B \mid\left(B, A, M^{\prime}\right) \in\right.$ get_cls $(r-1)$ for some $B$ and $\left.M^{\prime}\right\}$

$\xi:=\left\{c \in R^{+} \mid M(c)=c\right\}$

Construct $\Phi$ such that $\Phi \dot{\cup}\{M(c) \mid c \in \Phi\}=R^{+} \backslash \xi$.

if $\Psi_{1}=\{\}$ then

$$
\Psi_{1}:=\{\emptyset\}
$$

else

$$
\begin{aligned}
& \xi:=\left\{c \in \xi \mid c \cdot e_{r} \neq 0\right\} \\
& \Phi:=\left\{c \in \Phi \mid c \cdot e_{r} \neq 0\right\} \\
& \Psi_{2}:=\operatorname{seek} \_ \text {bases }(r, \xi) \\
& \Psi_{3}:=\text { seek_bases }(r, \Phi) \\
& \text { for }\left(B_{1}, B_{2}, B_{3}\right) \in \Psi_{1} \times \Psi_{2} \times \Psi_{3} \text { do } \\
& B:=B_{1} \cup B_{2} \cup B_{3} \cup\left\{M(c) \mid c \in B_{3}\right\} \\
& \text { if is_root_base }(B) \text { and } \mathfrak{C}(B, M) \nsubseteq \mathfrak{C}\left(B^{\prime}, M\right) \text { for all }\left(B^{\prime}, A, M\right) \in \Psi \text { then } \\
& \Psi:=\Psi \cup\{(B, A, M)\}
\end{aligned}
$$

return $\Psi$

Remark 5. The Dynkin diagrams of the root bases in the outputs of Algorithm 3 and Algorithm 4 for input $1 \leq r \leq 8$, are stated in [33, Corollary 2.1] and [6, Chapter 8]. The Dynkin diagrams of the root bases in the output of Algorithm 3 can alternatively be obtained using the procedure of Borel-Dynkin-Siebenthal (see [6, Section 8.2.3 and Section 8.7.2] for further references). 
Example 1. We consider the output of Algorithm 5 with input $r=3$. The corresponding row numbers are 3 until 14 in Table 7 . In Table 5 we depict the Cremona invariant $\mathfrak{C}(X)$ for each $[\mathbf{N}(X)]$ in the output of Algorithm 5 .

Recall from Proposition 1 and Notation 3 that

- $N(X) \cong\left\langle e_{0}, e_{1}, e_{2}, e_{3}\right\rangle_{\mathbb{Z}}$ with $-k=h=3 e_{0}-e_{1}-e_{2}-e_{3}$,

- $E(X)=\left\{e_{1}, e_{2}, e_{3}, e_{0}-e_{1}-e_{2}, e_{0}-e_{1}-e_{3}, e_{0}-e_{2}-e_{3}\right\}$,

- $F(X)=\left\{e_{0}-e_{1}, e_{0}-e_{2}, e_{0}-e_{3}\right\}$, and

- $R^{+}=\left\{e_{1}-e_{2}, e_{2}-e_{3}, e_{1}-e_{3}, e_{0}-e_{1}-e_{2}-e_{3}\right\}$.

We know from Lemma 4 and Lemma 6 that $G(X)=F_{\mathbb{R}}(X)$ and $A(X), B(X) \subset R^{+}$. Notice that $B(X)$ can be recovered from the square vertices in Table 5.

Table 5: The twelve Cremona invariants of Neron-Severi lattices of weak del Pezzo surfaces of degree 6 (self-loops of vertices are omitted).

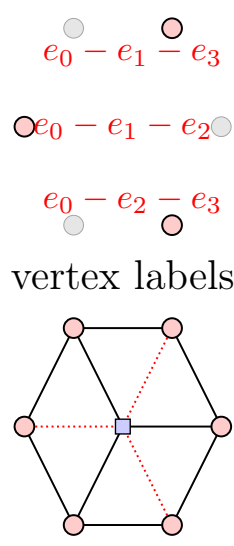

$\left(A_{0}, \underline{A_{1}}\right)$

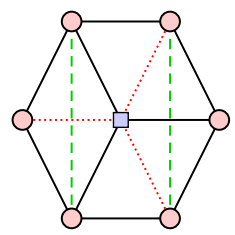

$\left(A_{1}, \underline{A_{1}}\right)$

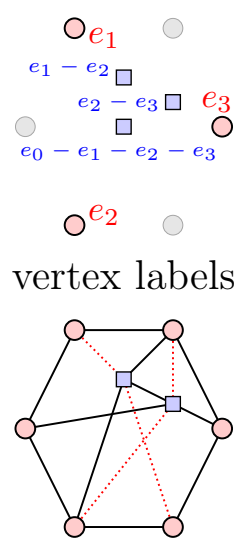

$\left(A_{0}, \underline{A_{2}}\right)$

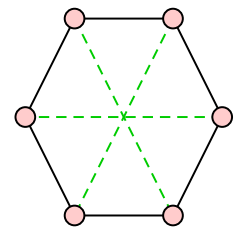

$\left(A_{1}^{\prime}, A_{0}\right)$

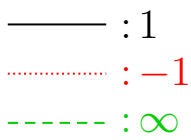

edge labels

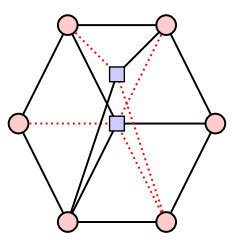

$\left(A_{0}, 2 \underline{A_{1}}\right)$

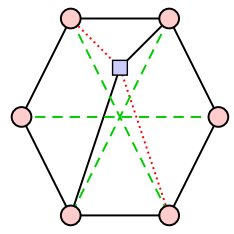

$\left(A_{1}^{\prime}, \underline{A_{1}}\right)$

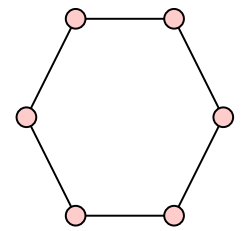

$\left(A_{0}, A_{0}\right)$

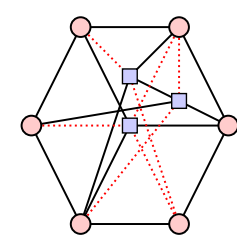

$\left(A_{0}, \underline{A_{1}}+\underline{A_{2}}\right)$

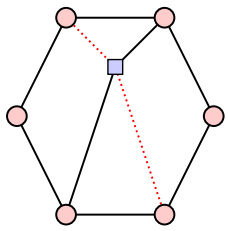

$\left(A_{0}, \underline{A_{1}}\right)$

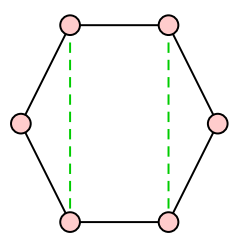

$\left(A_{1}, A_{0}\right)$

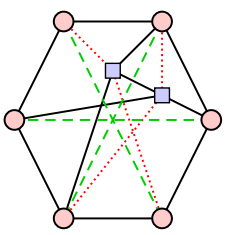

$\left(A_{1}^{\prime}, \underline{A_{2}}\right)$

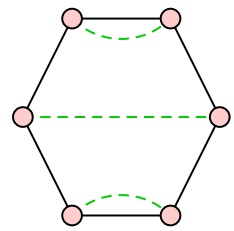

$\left(2 A_{1}, A_{0}\right)$

The output of Algorithm 3 with $r=3$ consists of $\emptyset,\left\{e_{1}-e_{2}\right\},\left\{e_{0}-e_{1}-e_{2}-e_{3}\right\}$, $\left\{e_{1}-e_{2}, e_{2}-e_{3}\right\},\left\{e_{1}-e_{2}, e_{0}-e_{1}-e_{2}-e_{3}\right\}$ and $\left\{e_{1}-e_{2}, e_{2}-e_{3}, e_{0}-e_{1}-e_{2}-e_{3}\right\}$.

The output of Algorithm 4 with $r=3$ is a set consisting of the following pairs (here the matrix $M$ is represented as $\left.\left[\sigma_{*}\left(e_{0}\right), \sigma_{*}\left(e_{1}\right), \sigma_{*}\left(e_{2}\right), \sigma_{*}\left(e_{3}\right)\right]\right)$ :

- $\left(\emptyset,\left[e_{0}, e_{1}, e_{2}, e_{3}\right]\right)$,

- $\left(\left\{e_{1}-e_{2}\right\},\left[e_{0}, e_{2}, e_{1}, e_{3}\right]\right)$, 
- $\left(\left\{e_{0}-e_{1}-e_{2}-e_{3}\right\},\left[2 e_{0}-e_{1}-e_{2}-e_{3}, e_{0}-e_{2}-e_{3}, e_{0}-e_{1}-e_{3}, e_{0}-e_{1}-e_{2}\right]\right)$,

- $\left(\left\{e_{1}-e_{2}, e_{0}-e_{1}-e_{2}-e_{3}\right\},\left[2 e_{0}-e_{1}-e_{2}-e_{3}, e_{0}-e_{1}-e_{3}, e_{0}-e_{2}-e_{3}, e_{0}-e_{1}-e_{2}\right]\right)$,

where $\mathfrak{D}(A(X))$ is $A_{0}, A_{1}, A_{1}^{\prime}$ and $2 A_{1}$, respectively. Notice that $\mathfrak{D}(A(X))$ does not uniquely determine $\sigma_{*}$ up to conjugacy, so we added a '-symbol in case $\sigma_{*}\left(e_{0}\right) \neq e_{0}$. If $A=\left\{e_{1}-e_{2}, e_{2}-e_{3}, e_{0}-e_{1}-e_{2}-e_{3}\right\}$ at step (1) in Algorithm 4, then the kernel of $A \cdot J$ has basis $\left\{e_{0}-e_{1}-e_{2}-e_{3}, k\right\}$ so that at step (2):

$$
Q=\left[\begin{array}{cccc}
0 & 0 & 1 & -3 \\
1 & 0 & -1 & 1 \\
-1 & 1 & -1 & 1 \\
0 & -1 & -1 & 1
\end{array}\right], \quad D=\left[\begin{array}{cccc}
-1 & 0 & 0 & 0 \\
0 & -1 & 0 & 0 \\
0 & 0 & -1 & 0 \\
0 & 0 & 0 & 1
\end{array}\right], \quad M=Q \cdot D \cdot Q^{-1}=\left[\begin{array}{cccc}
2 & 1 & 1 & 1 \\
-1 & -\frac{4}{3} & -\frac{1}{3} & -\frac{1}{3} \\
-1 & -\frac{1}{3} & -\frac{4}{3} & -\frac{1}{3} \\
-1 & -\frac{1}{3} & -\frac{1}{3} & -\frac{4}{3}
\end{array}\right]
$$

Thus $M$ does in this case not define a unimodular involution and is not added to the output $\Psi$ at step (3). When we follow Algorithm 5 with the output of Algorithm 4 we recover Table 5 and rows 3 until 14 in Table 7. For example, suppose that $(A, M)=\left(\left\{e_{1}-e_{2}\right\},\left[e_{0}, e_{2}, e_{1}, e_{3}\right]\right)$ in the outer for-loop of Algorithm 5. We find that get_cls $(2)=\left\{(\emptyset, \emptyset, I),\left(e_{1}-e_{2}, \emptyset, I\right),\left(\emptyset, e_{1}-e_{2},\left[e_{0}, e_{2}, e_{1}\right]\right)\right\}$ so that $\Psi_{1}=\{\emptyset\} \neq \emptyset$. We have $\xi=\left\{e_{0}-e_{1}-e_{2}-e_{3}\right\}$ and $\Phi=\left\{e_{1}-e_{3}\right\}$ where $M\left(e_{1}-e_{3}\right)=e_{2}-e_{3}$. Thus $\Psi_{2}=\left\{\emptyset, e_{0}-e_{1}-e_{2}-e_{3}\right\}$ and $\Psi_{3}=\left\{\emptyset, e_{1}-e_{3}\right\}$. If $\left(B_{1}, B_{2}, B_{3}\right)=\left(\emptyset, \emptyset, e_{1}-e_{3}\right)$, then $B$ is not a root base, since $\left(e_{1}-e_{3}\right) \cdot\left(e_{2}-e_{3}\right) \notin\{0,1\}$.

Lemma 7. The output specification of Algorithm 5 is correct.

Proof. The correctness of both Algorithm 1 and Algorithm 2 is a direct consequence of the definitions. It follows from Lemma 6 that we only have to consider roots in $R^{+}$. Notice that the columns of $Q$ at step 1 of Algorithm 4 are eigenvectors of $M$ so that $M \cdot Q=D \cdot Q$. The correctness of Algorithm 4 is thus a straightforward consequence of Proposition 3d and Remark 4.

For Algorithm 3 we recall from Lemma 2 that if $Y \rightarrow X$ is the smooth model of a weak del Pezzo surface $X$, then $Y$ is the complex blowup of a weak del Pezzo surface $X^{\prime}$. Thus by Proposition $1 \mathrm{a}, N(X) \cong N\left(X^{\prime}\right) \oplus\left\langle e_{r}\right\rangle_{\mathbb{Z}}, N\left(X^{\prime}\right) \cong\left\langle e_{0}, \ldots, e_{r-1}\right\rangle_{\mathbb{Z}}$ and $B(X)$ is the union of $B\left(X^{\prime}\right)$ (represented by $B_{1}$ ) with $B_{2} \subset\left\{c \in R(X) \mid c \cdot e_{r} \neq\right.$ $0\}$. We know from Proposition $3 \mathrm{~b}$ that $B(X)$ and $B\left(X^{\prime}\right)$ are root bases. Thus $B_{2}$ is a root base as well and the correctness of Algorithm 3 follows.

For Algorithm 5 we apply the same argument as we used for Algorithm 3. However, in this case $B\left(X^{\prime}\right) \subset B(X)$ only if $\sigma_{*}\left(e_{r}\right)=e_{r}$, which means that $\Psi_{1} \neq \emptyset$. Notice 
that $A(X)$ determines $\sigma_{*}$ only up to a choice of a basis for the sublattice $\{c \in$ $N(X) \mid c \cdot a=0$ for all $a \in A(X)\}$. It follows from Lemma 6 that $B(X) \subset R^{+}$ for any such choice of $\sigma_{*}$. However, we need to exhaust all possible root bases $B(X) \subset R^{+}$such that $\sigma_{*}(B(X))=B(X)$. Any such candidate $B$ for $B(X)$ can be constructed from some $\left(B_{1}, B_{2}, B_{3}\right) \in \Psi_{1} \times \Psi_{2} \times \Psi_{3}$. We know from Lemma 5 that $B(X)$ uniquely determines $h^{0}: N(X) \rightarrow \mathbb{Z}_{\geq 0}$. We conclude from Proposition 4 that the output specification of Algorithm 5 is correct.

Proof of Theorem 2. Notice that Lemma 4[a,b,c,d,e,f] correspond to the rows (i), (ii), (iii), (iv), (v) and (2) in Table 7, respectively. By Proposition 1c, we can compute $E_{\mathbb{R}}(X)$ and $F_{\mathbb{R}}(X)$ from $B(X)$. Thus the proof is a direct consequence of Lemma 4 and Lemma 7. It follows from [6, Section 8.7.1 and Theorem 8.8.1] that if $X$ has canonical degree $\leq 2$, then $\mathbf{N}(X)$ is not characterized by row $176,434,453$, 455 or 505 .

We explain in the following example how to read Table 7.

Example 2. Suppose that $X \subset \mathbb{P}^{3}$ is a ring cyclide as in Figure 1 so that $X$ is a quartic weak del Pezzo surface that is covered by four simple families of conics and $X$ contains neither real lines nor real isolated singularities. It follows from Theorem 2 that $\mathbf{N}(X)$ is characterized by a row in Table 7 . The table headers $d$, $D(A), D(B), \# E$ and $\# G$ correspond to $\operatorname{deg} X, \mathfrak{D}(A(X)), \mathfrak{D}(B(X)),\left|E_{\mathbb{R}}(X)\right|$ and $|G(X)|$, respectively. The components of $\mathfrak{D}(B(X))$ that are preserved by $\sigma_{*}$ are underlined in table. It follows from Proposition 2 that $d=4$, the $D(B)$ entry has no underlined components, $\# E=0$ and $\# G=4$. Hence, $\mathbf{N}(X)$ must be defined by row number 67 such that $\mathfrak{D}(A(X))=2 A_{1}^{\prime}$ and $\mathfrak{D}(B(X))=4 A_{1}$. It follows from Proposition 1a that $N(X) \cong\left\langle e_{0}, \ldots, e_{5}\right\rangle_{\mathbb{Z}}$ and $-k=h=3 e_{0}-e_{1}-\ldots-e_{5}$. We recover an explicit description for $\sigma_{*}: N(X) \rightarrow N(X)$ and $B(X)$ from the entry $\frac{9}{5} \frac{3}{6} \frac{3}{5} \frac{3}{4} \frac{0}{5} \frac{0}{4} \| \frac{1}{1} \frac{1}{6} \frac{3}{9} \frac{4}{2}$, which can be decoded using the dictionary at Table 7 . We find that $\sigma_{*}\left(e_{0}\right)=2 e_{0}-e_{1}-e_{2}-e_{3}, \sigma_{*}\left(e_{1}\right)=e_{0}-e_{2}-e_{3}, \sigma_{*}\left(e_{2}\right)=e_{0}-e_{1}-e_{3}, \sigma_{*}\left(e_{3}\right)=$ $e_{0}-e_{1}-e_{2}, \sigma_{*}\left(e_{4}\right)=e_{5}$ and $B(X)=\left\{e_{3}-e_{4}, e_{2}-e_{5}, e_{0}-e_{1}-e_{3}-e_{4}, e_{0}-e_{1}-e_{2}-e_{5}\right\}$. Recall from Lemma 5 that $B(X)$ uniquely recovers $h^{0}: N(X) \rightarrow \mathbb{Z}_{\geq 0}$ and thus we determined $\mathbf{N}(X)$ up to isomorphism of Neron-Severi lattices. We have $E_{\star}(X)=$ $\left\{e_{1}, e_{0}-e_{2}-e_{3}, e_{4}, e_{5}\right\}$ and $F_{\mathbb{R}}(X)=\left\{e_{0}-e_{1}, e_{0}-e_{2}, e_{0}-e_{3}, 2 e_{0}-e_{2}-e_{3}-e_{4}-e_{5}\right\}$, by Proposition 1 , and we know that $G(X)=F_{\mathbb{R}}(X)$ by Lemma 4 . Recall from 
Remark 3 that the simple family graph $\mathcal{G}(X)$ in Figure 1 is uniquely determined by the incidence diagram $\mathfrak{D}(G(X))$. Notice that $e_{0}-e_{1}$ and $2 e_{0}-e_{2}-e_{3}-e_{4}-e_{5}$ are the classes of Villarceau circles.

Remark 6. Suppose that $X$ and $X^{\prime}$ are anticanonical models of smooth weak del Pezzo surfaces. It is observed in [33, Section 2] that $\mathbf{N}(X) \cong \mathbf{N}\left(X^{\prime}\right)$ does not imply that $\mathfrak{D}(A(X)) \cong \mathfrak{D}\left(A\left(X^{\prime}\right)\right)$. Indeed, using the Cremona invariant we find that if $\operatorname{deg} X=\operatorname{deg} X^{\prime}$ and both $\mathfrak{D}(A(X))$ and $\mathfrak{D}\left(A\left(X^{\prime}\right)\right)$ are listed below at the same bullet, then $\mathbf{N}(X) \cong \mathbf{N}\left(X^{\prime}\right)$ :

- $4 A_{1}, D_{4}$.

- $5 A_{1}, A_{1}+D_{4}$.

- $6 A_{1}, 2 A_{3}, 2 A_{1}+D_{4}, D_{6}$.

- $7 A_{1}, A_{7}, A_{2}+A_{5}, A_{1}+D_{6}, 3 A_{1}+D_{4}, A_{1}+2 A_{3}, E_{7}$.

- $8 A_{1}, A_{8}, 4 A_{2}, 2 A_{4}, 2 D_{4}, A_{3}+D_{5}, A_{1}+A_{7}, A_{2}+E_{6}, A_{1}+E_{7}, 4 A_{1}+D_{4}, 2 A_{1}+D_{6}$, $2 A_{1}+2 A_{3}, A_{1}+A_{2}+A_{5}, D_{8}, E_{8}$.

\section{Simple family graphs via adjunction}

In this section we prove Theorem 1 and Theorem 4 . We start by recalling a version of adjunction as defined in [18, Section 2.4].

A ruled pair is defined as a pair $(Y, h)$ where $Y$ is a smooth birationally ruled surface, $h$ is nef and there are no classes of complex $(-1)$-curves that are orthogonal to $h$.

Suppose that $Y_{0} \rightarrow X$ is the smooth model of a surface $X \subset \mathbb{P}^{n}$ that is covered by a simple family. Let $h_{0} \in N(X)$ be the class of hyperplane sections. In this case, $\left(Y_{0}, h_{0}\right)$ is a ruled pair. If $h^{0}\left(h_{0}+k_{0}\right)>1$, then we consider the birational morphism $\mu: Y_{0} \rightarrow Y_{1}$ that contracts all complex $(-1)$-curves $E \subset Y_{0}$ such that $\left(h_{0}+k_{0}\right) \cdot[E]=0$. An adjoint relation is defined as

$$
\left(Y_{0}, h_{0}\right) \stackrel{\mu}{\longrightarrow}\left(Y_{1}, h_{1}\right):=\left(\mu\left(Y_{0}\right), \mu_{*}\left(h_{0}+k_{0}\right)\right) .
$$

This relation $\mu$ is unique up to biregular isomorphism and $\left(Y_{1}, h_{1}\right)$ is again a ruled pair [18, Proposition 1]. In particular, the adjoint relation satisfies the following property: 
Lemma 8. If $(Y, h) \stackrel{\mu}{\longrightarrow}\left(Y^{\prime}, h^{\prime}\right)$ is an adjoint relation such that $h^{\prime 2}>0$ and $E, E^{\prime} \subset$ $Y$ are complex $(-1)$-curves that are contracted by $\mu: Y \rightarrow Y^{\prime}$, then $[E] \cdot\left[E^{\prime}\right]=0$.

Proof. We have that $\mu^{*} h^{\prime}=h+k$, since the classes of complex $(-1)$-curves that are contracted by $\mu$ are by definition orthogonal to $h+k$. Thus $(h+k)^{2}=\left(\mu^{*} h^{\prime}\right)^{2}=$ $h^{\prime 2}>0$. Since $(h+k) \cdot\left([E]+\left[E^{\prime}\right]\right)=0$ it follows from $(\mathrm{HI})$ that $\left([E]+\left[E^{\prime}\right]\right)^{2}<0$ and thus $[E] \cdot\left[E^{\prime}\right]=0$.

The adjoint chain for $X$ is a chain of adjoint relations

$$
\left(Y_{0}, h_{0}\right) \stackrel{\mu_{0}}{\longrightarrow}\left(Y_{1}, h_{1}\right) \stackrel{\mu_{1}}{\longrightarrow} \ldots \stackrel{\mu_{\ell-1}}{\longrightarrow}\left(Y_{\ell}, h_{\ell}\right)
$$

such that $h^{0}\left(h_{i}+k_{i}\right)>1$ for $0 \leq i<\ell$ and $h^{0}\left(h_{\ell}+k_{\ell}\right) \leq 1$. Although $Y_{i}$ for $0 \leq i \leq \ell$ is considered as an abstract surface it comes equiped with the class of hyperplane sections $h_{i}$ and the canonical class $k_{i}$. This motivates us to consider the following definition for the classes of simple families of $Y_{i}$ :

$$
G\left(Y_{i}\right):=\left\{c \in N\left(Y_{i}\right) \mid p_{a}(c)=0, h^{0}(c) \geq 2 \text { and } h_{i} \cdot c=\nu_{i}\right\}
$$

where $\nu_{i}:=\min \left\{h_{i} \cdot c \mid c \in N\left(Y_{i}\right), p_{a}(c)=0\right.$ and $\left.h^{0}(c) \geq 2\right\}$. Notice that $G(X)=$ $G\left(Y_{0}\right)$ by definition. Recall from Remark 3 that we recover the simple family graph $\mathcal{G}(X)$ uniquely from $G(X)$.

The following Lemma 9 and Lemma 10 collect results from [18]. We use the following notation for $t \in \mathbb{Z}:[t]:=1$ and $[t]:=0$ if $t \geq 0$ and $t<0$, respectively.

Lemma 9. If $\left(Y_{0}, h_{0}\right) \stackrel{\mu_{0}}{\longrightarrow}\left(Y_{1}, h_{1}\right) \stackrel{\mu_{1}}{\longrightarrow} \ldots \stackrel{\mu_{\ell-1}}{\longrightarrow}\left(Y_{\ell}, h_{\ell}\right)$ is an adjoint chain, then either $G\left(Y_{0}\right)=\{f\}$ such that $k_{0} \cdot f=-2$, or $Y_{\ell}$ is a weak del Pezzo surface and one of the following four cases holds:

a. $G\left(Y_{0}\right)=\left\{\left(\mu_{\ell-1} \circ \ldots \circ \mu_{0}\right)^{*}(g) \mid g \in G\left(Y_{\ell}\right), k_{\ell} \cdot g=-2\right\} \neq \emptyset$.

b. $N\left(Y_{i}\right)$ admits a real type 1 basis for all $0 \leq i \leq \ell$ such that

$$
\begin{aligned}
h_{i} & =\left(\alpha_{0}-3 i\right) e_{0}-\left[\alpha_{1}-i\right]\left(\alpha_{1}-i\right) e_{1}-\ldots-\left[\alpha_{r}-i\right]\left(\alpha_{r}-i\right) e_{r}, \\
k_{i} & =-3 e_{0}+\left[\alpha_{1}-i\right] e_{1}+\ldots+\left[\alpha_{r}-i\right] e_{r}
\end{aligned}
$$

where $\alpha_{0}-3 i>0$ and $\alpha_{1}, \ldots, \alpha_{r} \in \mathbb{Z}_{>0}$. Moreover, $c \in G\left(Y_{i}\right)$ is up to permutation of $\left(e_{j}\right)_{j>0}$ an element of $\left\{e_{0}, e_{0}-e_{1}, 2 e_{0}-e_{1}-e_{2}-e_{3}-e_{4}\right\}$. 
c. $N\left(Y_{i}\right)$ admits a real type 2 basis for all $0 \leq i \leq \ell$ such that

$$
\begin{aligned}
h_{i} & =\left(\alpha_{0}-2 i\right)\left(\ell_{0}+\ell_{1}\right)-\left[\alpha_{1}-i\right]\left(\alpha_{1}-i\right) \varepsilon_{1}-\ldots-\left[\alpha_{r}-i\right]\left(\alpha_{r}-i\right) \varepsilon_{r}, \\
k_{i} & =-2\left(\ell_{0}+\ell_{1}\right)+\left[\alpha_{1}-i\right] \varepsilon_{1}+\ldots+\left[\alpha_{r}-i\right] \varepsilon_{r},
\end{aligned}
$$

where $\alpha_{0}-2 i>0$ and $\alpha_{1}, \ldots, \alpha_{r} \in \mathbb{Z}_{>0}$. Moreover, $c \in G\left(Y_{i}\right)$ is up to permutation of $\left(\varepsilon_{j}\right)_{j>0}$ an element of $\left\{\ell_{0}+\ell_{1}, \ell_{0}+\ell_{1}-\varepsilon_{1}, \ell_{0}+\ell_{1}-\varepsilon_{1}-\varepsilon_{2}\right\}$.

d. $G\left(Y_{0}\right)=G\left(Y_{\ell}\right)=\emptyset$ and $Y_{\ell}$ is not $\mathbb{R}$-rational.

Proof. See [18, Lemma 1 and Lemma 2] for the case distinction (this result is an adaptation of the classification result by Comessatti [30, Theorem 4.6]). For case (a) see also [18, Proposition 3 or Theorem 1i]. For the candidate classes at (b) and (c) see [18, Theorem 1ii and Theorem 1iii].

Lemma 10. Let $\left(Y_{0}, h_{0}\right) \stackrel{\mu_{0}}{\longrightarrow}\left(Y_{1}, h_{1}\right) \stackrel{\mu_{1}}{\longrightarrow} \ldots \stackrel{\mu_{\ell-1}}{\longrightarrow}\left(Y_{\ell}, h_{\ell}\right)$ be an adjoint chain.

a) If $Y_{i} \cong \mathbb{P}^{2}$ for some $0<i \leq \ell$ and $\mu_{i-1}$ contracts at least one real $(-1)$-curve, then $G\left(Y_{i-1}\right)=\left\{e_{0}-e_{j} \mid \mu_{*}\left(e_{j}\right)=e_{j}\right.$ for $\left.1 \leq j \leq r\right\}$.

b) If $G\left(Y_{i}\right)=\left\{g \in G\left(Y_{i}\right) \mid k_{i} \cdot g=-2\right\}$ for some $0<i \leq \ell$, then $G\left(Y_{i-1}\right)=$ $\left\{\mu_{i-1}^{*}(g) \mid g \in G\left(Y_{i}\right)\right.$ and $\left.k_{i} \cdot g=-2\right\}$.

c) $h^{0}(g)=-k_{0} \cdot g-1$ for all $g \in G\left(Y_{0}\right)$.

Proof. For a), b) and c), see [18, Lemma 12], [18, Proposition 3] and [18, Corollary $1 \mathrm{~b}]$, respectively.

Lemma 11. Let $(Y, h)$ be a ruled pair.

a) Suppose that $N(Y)$ admits a real type 1 basis such that in addition $\sigma_{*}\left(e_{1}\right)=e_{2}$ and $\sigma_{*}\left(e_{3}\right)=e_{4}$. If $h \cdot g<h \cdot e_{0}$ where $g:=2 e_{0}-e_{1}-e_{2}-e_{3}-e_{4}$, then $g$ is the class of a complete rational family.

b) Suppose that $N(Y)$ admits a real type 2 basis such that in addition $\sigma_{*}\left(\ell_{0}\right)=\ell_{1}$ and $\sigma_{*}\left\{\varepsilon_{1}, \varepsilon_{2}\right\}=\left\{\varepsilon_{1}, \varepsilon_{2}\right\}$. In this case $f:=\ell_{0}+\ell_{1}-\varepsilon_{1}-\varepsilon_{2}$ is the class of $a$ complete rational family. 
Proof. a) We consider $g$ as the class of the linear series $|g|$ of conics in $\mathbb{P}^{2}$ that pass through four base points. Suppose by contradiction that $|g|$ has a fixed component. If $h^{0}\left(e_{0}-e_{1}-e_{2}-e_{3}\right)=1$, then $h^{0}\left(e_{0}-e_{1}-e_{2}-e_{3}-e_{4}\right)=1$ as well, since $\sigma_{*}\left(e_{3}\right)=e_{4}$ by assumption. It follows that $e_{0}$ is the class of the moving component and $e_{0}-e_{1}-$ $e_{2}-e_{3}-e_{4}$ is the class of the fixed component so that $h^{0}\left(e_{0}-e_{1}-e_{2}-e_{3}-e_{4}\right)=1$. Since $h \cdot g<h \cdot e_{0}$ we find that $h \cdot\left(e_{0}-e_{1}-e_{2}-e_{3}-e_{4}\right)<0$. We arrived at a contradication as $h$ is nef. Therefore $|g|$ has no fixed components and thus $g$ is the class of a complete rational family.

b) We consider $f$ as the class of the linear series $|f|$ of bidegree $(1,1)$ forms on $\mathbb{P}^{1} \times \mathbb{P}^{1}$ that pass through two base points. If $|f|$ would have a fixed component, then without loss of generality $\ell_{0}$ is the class of the moving component and $\ell_{1}-\varepsilon_{1}-\varepsilon_{2}$ is the class of the fixed component. However, this implies that $\sigma_{*}\left(\ell_{0}\right)=\ell_{0}$ and thus the linear series of $f$ has no fixed components. We conclude from (AF) that $f$ is as asserted.

Notation 4. We consider the following types of real abstract surfaces:

$\mathbf{P}_{0}: \mathbb{P}^{2}$ together with its unique real structure.

$\mathbf{P}_{1}: \mathbb{P}^{2}$ blownup in at least 1 real point.

$\mathbf{P}_{2}: \mathbb{P}^{2}$ blownup in exactly 2 complex conjugate points.

$\mathbf{P}_{4}: \mathbb{P}^{2}$ blownup in at least 4 complex conjugate points and no real points.

$\mathbf{S}_{0}: \mathbb{P}^{1} \times \mathbb{P}^{1}$ together with the real structure $\sigma$ that flips the two factors.

$\mathbf{S}_{1}: \mathbf{S}_{0}$ blown up in exactly 1 real point.

$\mathbf{S}_{2}: \mathbf{S}_{0}$ blown up in at least 2 complex points.

For example, we write $Y_{0} \in \mathbf{P}_{1}$ if $Y_{0}$ is the blowup of $\mathbb{P}^{2}$ in at least 1 real point; we use the same notation for the other cases.

Lemma 12. Let $\left(Y_{0}, h_{0}\right) \stackrel{\mu_{0}}{\longrightarrow}\left(Y_{1}, h_{1}\right) \stackrel{\mu_{1}}{\longrightarrow} \ldots \stackrel{\mu_{\ell-1}}{\longrightarrow}\left(Y_{\ell}, h_{\ell}\right)$ be an adjoint chain. If $Y_{\ell}$ is a weak del Pezzo surface, $\left\{g \in G\left(Y_{\ell}\right) \mid k_{\ell} \cdot g=-2\right\}=\emptyset$ and $G\left(Y_{\ell}\right) \neq \emptyset$, then $N\left(Y_{0}\right)$ is generated by either a real type 1 basis or a real type 2 basis and one of the 8 cases in Table 6[a-h] holds.

Before we prove Lemma 12 and Theorem 1 let us first consider some explicit examples. 
Table 6: See Lemma 12 and Notation 4.

Notation:

$$
\begin{aligned}
& \alpha:=\max \left(\left\{0 \leq i<\ell \mid Y_{i} ¥ Y_{\ell}\right\} \cup\{-1\}\right) ; \text { if } \alpha=-1, \text { then } \alpha:=\ell . \\
& \beta:=\max \left(\left\{0 \leq i \leq \ell \mid G\left(Y_{i}\right)=\left\{g \in G\left(Y_{i}\right) \mid k_{i} \cdot g=-2\right\}\right\} \cup\{0\}\right) . \\
& \tau:=\operatorname{rank} N\left(Y_{\beta}\right)-1 . \\
& \xi_{1}:=\left\{e_{0}-e_{a} \mid \sigma_{*} e_{a}=e_{a}, 0<a \leq \tau\right\} . \\
& \xi_{2}:=\left\{2 e_{0}-e_{1}-e_{2}-e_{a}-e_{b} \mid \sigma_{*} e_{a}=e_{b}, 3 \leq a<b \leq \tau\right\} . \\
& \xi_{3}:=\left\{2 e_{0}-e_{a}-e_{b}-e_{c}-e_{d} \mid \sigma_{*} e_{a}=e_{b}, \sigma_{*} e_{c}=e_{d}, 0<a<b<c<d \leq \tau\right\} . \\
& \xi_{4}:=\left\{\ell_{0}+\ell_{1}-\varepsilon_{1}-\varepsilon_{a} \mid \sigma_{*} \varepsilon_{a}=\varepsilon_{a}, 0<a \leq \tau\right\} . \\
& \xi_{5}:=\left\{\ell_{0}+\ell_{1}-\varepsilon_{a}-\varepsilon_{b} \mid \sigma_{*}\left\{\varepsilon_{a}, \varepsilon_{b}\right\}=\left\{\varepsilon_{a}, \varepsilon_{b}\right\}, 0<a<b \leq \tau\right\} . \\
& \xi_{2}^{\prime} \subseteq \xi_{2} \text { and } \xi_{3}^{\prime} \subseteq \xi_{3} .
\end{aligned}
$$

Cases:

a. $Y_{\alpha} \in \mathbf{P}_{1}, Y_{\ell} \in \mathbf{P}_{0}$ and $G\left(Y_{0}\right)=\xi_{1}$.

b. $Y_{0} \in \mathbf{P}_{2}$ or $Y_{0} \in \mathbf{P}_{0}$, and $G\left(Y_{0}\right)=\left\{e_{0}\right\}$.

c. $Y_{0} \notin \mathbf{P}_{2}, Y_{\alpha} \in \mathbf{P}_{2}$ and $G\left(Y_{0}\right) \in\left\{\xi_{1}, \xi_{2}, \xi_{2}^{\prime} \cup\left\{e_{0}\right\}, \xi_{2} \cup \xi_{1}\right\}$.

d. $Y_{\alpha} \in \mathbf{P}_{4}, Y_{\ell} \in \mathbf{P}_{0}$ and $G\left(Y_{0}\right)=\left\{\xi_{3}, \xi_{3}^{\prime} \cup\left\{e_{0}\right\}, \xi_{3} \cup \xi_{1}\right\}$.

e. $Y_{0} \in \mathbf{S}_{0}$ and $G\left(Y_{0}\right)=\left\{\ell_{0}+\ell_{1}\right\}$.

f. $Y_{0} \in \mathbf{S}_{1}$ and $G\left(Y_{0}\right)=\left\{\ell_{0}+\ell_{1}-\varepsilon_{1}\right\}$.

g. $Y_{0} \in \mathbf{S}_{2}, Y_{\alpha} \in \mathbf{S}_{1}$ and $G\left(Y_{0}\right) \in\left\{\xi_{4}, \xi_{5} \cup\left\{\ell_{0}+\ell_{1}-\varepsilon_{1}\right\},\left\{\ell_{0}+\ell_{1}-\varepsilon_{1}\right\}\right\}$.

h. $Y_{\alpha} \in \mathbf{S}_{2}, Y_{\ell} \in \mathbf{S}_{0}$ and $G\left(Y_{0}\right)=\xi_{5}$.

Example 3 (Theorem 1f). Suppose that $\left(Y_{0}, h_{0}\right) \stackrel{\mu_{0}}{\longrightarrow}\left(Y_{1}, h_{1}\right)$ is the adjoint chain of a surface $X \subset \mathbb{P}^{n}$. The involution $\sigma_{*}: N\left(Y_{0}\right) \rightarrow N\left(Y_{0}\right)$ is defined as $\sigma_{*}\left(e_{0}\right)=e_{0}$ and $\sigma_{*}\left(e_{i}\right)=e_{i+1}$ for $i \in\{1,3,5,7\}$; and

- $Y_{1} \in \mathbf{P}_{0}, h_{1}=e_{0}, k_{1}=-3 e_{0}$ and $G\left(Y_{1}\right)=\left\{e_{0}\right\}$.

- $Y_{0} \in \mathbf{P}_{4}, h_{0}=4 e_{0}-e_{1}-\ldots-e_{8}, k_{0}=-3 e_{0}+e_{1}+\ldots+e_{8}$ and $G\left(Y_{0}\right)=\left\{e_{0}\right.$,

$$
\begin{aligned}
& 2 e_{0}-e_{1}-e_{2}-e_{3}-e_{4}, 2 e_{0}-e_{1}-e_{2}-e_{5}-e_{6}, 2 e_{0}-e_{1}-e_{2}-e_{7}-e_{8}, 2 e_{0}-e_{3}-e_{4}-e_{5}-e_{6}, \\
& \left.2 e_{0}-e_{3}-e_{4}-e_{7}-e_{8}, 2 e_{0}-e_{5}-e_{6}-e_{7}-e_{8}\right\} .
\end{aligned}
$$

The adjoint chain is characterized by Table $6 \mathrm{~d}$ and the simple family graph $\mathcal{G}(X)$ is characterized by Theorem 1f. 
Example 4 (Theorem 1h). Suppose that $\left(Y_{0}, h_{0}\right) \stackrel{\mu_{0}}{\longrightarrow}\left(Y_{1}, h_{1}\right) \stackrel{\mu_{1}}{\longrightarrow}\left(Y_{2}, h_{2}\right)$ is the adjoint chain of a surface $X \subset \mathbb{P}^{n}$. The involution $\sigma_{*}: N\left(Y_{0}\right) \rightarrow N\left(Y_{0}\right)$ is defined as $\sigma_{*}\left(e_{0}\right)=e_{0}, \sigma_{*}\left(e_{i}\right)=e_{i+1}$ for $i \in\{1,3,5\}, \sigma_{*}\left(e_{7}\right)=e_{7}, \sigma_{*}\left(e_{8}\right)=e_{8}$ and $\sigma_{*}\left(e_{9}\right)=e_{9}$; and

- $Y_{2} \in \mathbf{P}_{2}, h_{2}=3 e_{0}-e_{1}-e_{2}, k_{2}=-3 e_{0}+e_{1}+e_{2}$ and $G\left(Y_{2}\right)=\left\{e_{0}\right\}$,

- $Y_{1} \in \mathbf{P}_{4}, h_{1}=6 e_{0}-2 e_{1}-2 e_{2}-e_{3}-e_{4}-e_{5}-e_{6}, k_{1}=-3 e_{0}+e_{1}+\ldots+e_{6}$ and $G\left(Y_{1}\right)=\left\{e_{0}, 2 e_{0}-e_{1}-e_{2}-e_{3}-e_{4}, 2 e_{0}-e_{1}-e_{2}-e_{5}-e_{6}\right\}$,

- $Y_{0} \notin \mathbf{P}_{2}, h_{0}=9 e_{0}-3 e_{1}-3 e_{2}-2 e_{3}-2 e_{4}-2 e_{5}-2 e_{6}-e_{7}-e_{8}-e_{9}, k_{0}=$ $-3 e_{0}+e_{1}+\ldots+e_{9}$, and $G\left(Y_{0}\right)=\left\{2 e_{0}-e_{1}-e_{2}-e_{3}-e_{4}, 2 e_{0}-e_{1}-e_{2}-e_{5}-e_{6}\right.$, $\left.e_{0}-e_{7}, e_{0}-e_{8}, e_{0}-e_{9}\right\}$.

The adjoint chain is characterized by Table $6 \mathrm{c}$ and $\mathcal{G}(X)$ is characterized by Theorem 1 h.

Example 5 (Theorem $1 \mathrm{j}$ ). Suppose that $\left(Y_{0}, h_{0}\right) \stackrel{\mu_{0}}{\longrightarrow}\left(Y_{1}, h_{1}\right)$ is the adjoint chain of a surface $X \subset \mathbb{P}^{n}$. The involution $\sigma_{*}: N\left(Y_{0}\right) \rightarrow N\left(Y_{0}\right)$ is defined as $\sigma_{*}\left(\ell_{0}\right)=\ell_{1}$, $\sigma_{*}\left(\varepsilon_{i}\right)=\varepsilon_{i}$ for $i \in\{1,2,3\}$ and $\sigma_{*}\left(\varepsilon_{i}\right)=\varepsilon_{i+1}$ for $i \in\{4,6,8\}$; and

- $Y_{1} \in \mathbf{S}_{0}, h_{1}=\ell_{0}+\ell_{1}, k_{1}=-2\left(\ell_{0}+\ell_{1}\right)$ and $G\left(X_{1}\right)=\left\{\ell_{0}+\ell_{1}\right\}$,

- $Y_{0} \in \mathbf{S}_{2}, h_{0}=3\left(\ell_{0}+\ell_{1}\right)-\varepsilon_{1}-\ldots-\varepsilon_{5}, k_{0}=-2\left(\ell_{0}+\ell_{1}\right)+\varepsilon_{1}+\ldots+\varepsilon_{5}$ and $G\left(X_{0}\right)=\left\{\ell_{0}+\ell_{1}-\varepsilon_{1}-\varepsilon_{2}, \ell_{0}+\ell_{1}-\varepsilon_{1}-\varepsilon_{3}, \ell_{0}+\ell_{1}-\varepsilon_{2}-\varepsilon_{3}, \ell_{0}+\ell_{1}-\varepsilon_{4}-\varepsilon_{5}\right.$, $\left.\ell_{0}+\ell_{1}-\varepsilon_{6}-\varepsilon_{7}, \ell_{0}+\ell_{1}-\varepsilon_{8}-\varepsilon_{9}\right\}$.

The adjoint chain is characterized by Table $6 \mathrm{~h}$ and $\mathcal{G}(X)$ is characterized by Theorem $1 \mathrm{j}$.

Proof of Lemma 12. Our strategy is to backtrace through the adjoint chain and determine all possible scenarios for $G\left(Y_{i}\right)$ for $i=\ell-1, \ell-2, \ldots, 0$ subsequently. Recall from Lemma 9 that $N\left(Y_{i}\right)$ admits a real type 1 or 2 basis and that the possible classes in $G\left(Y_{i}\right)$ are up to permutation of the $\left(e_{j}\right)_{j>0}$ and $\left(\varepsilon_{j}\right)_{j>0}$ in the set

$$
\left\{e_{0}, e_{0}-e_{1}, 2 e_{0}-e_{1}-e_{2}-e_{3}-e_{4}\right\} \cup\left\{\ell_{0}+\ell_{1}, \ell_{0}+\ell_{1}-\varepsilon_{1}, \ell_{0}+\ell_{1}-\varepsilon_{1}-\varepsilon_{2}\right\} .
$$

Moreover, we know $k_{i}$ and $h_{i}$ for $i>0$ if we know $h_{\ell}$ and the number of complex $(-1)$ curves that are contracted during each adjoint relation. Let $\Upsilon_{i}:=\left\{g \in G\left(Y_{i}\right) \mid k_{i}\right.$. $g=-2\}$ and recall that $\Upsilon_{\ell}=\emptyset$ by assumption 
We use the following observations:

- If $Y_{0} \cong Y_{\ell}$, then it follows from Lemma $4[\mathrm{a}, \mathrm{f}, \mathrm{c}]$ that $G\left(Y_{\ell}\right) \in\left\{\left\{e_{0}\right\},\left\{\ell_{0}+\ell_{1}\right\}\right\}$ and one of the cases in Table $6[\mathrm{~b}, \mathrm{e}]$ holds.

- If $Y_{i} ¥ \mathbb{P}^{2}$ and $Y_{i+1} \cong \mathbb{P}^{2}$, then either Table 6 a holds or $Y_{i} \in \mathbf{P}_{2} \cup \mathbf{P}_{4}$, by Lemma 10a. Moreover, $h_{\ell}=m e_{0}$ for some $m \in \mathbb{Z}_{>0}$.

- If $\Upsilon_{i} \neq \emptyset$, then $G\left(Y_{i}\right) \in\left\{\Upsilon_{i}, \Upsilon_{i} \cup\left\{e_{0}\right\}, \Upsilon_{i} \cup\left\{\ell_{0}+\ell_{1}-\varepsilon_{1}\right\}\right\}$ and if $G\left(Y_{i}\right)=\Upsilon_{i}$, then we may assume without loss of generality that $i=0$, by Lemma 10b.

- If $G\left(Y_{i}\right) \cap \xi_{1} \neq \emptyset$, then $e_{0} \notin G\left(Y_{i}\right)$ and thus $G\left(Y_{i}\right) \in\left\{\xi_{1}, \xi_{2} \cup \xi_{1}, \xi_{3} \cup \xi_{1}\right\}$ by Lemma 11a and one of Table $6[\mathrm{a}, \mathrm{c}, \mathrm{d}]$ holds.

- If $G\left(Y_{i}\right) \cap \xi_{3} \neq \emptyset$ and $e_{0} \notin G\left(Y_{i}\right)$, then $G\left(Y_{i}\right) \in\left\{\xi_{2}, \xi_{3}\right\}$ by Lemma 11a and one of Table $6[\mathrm{c}, \mathrm{d}]$ holds.

- If $G\left(Y_{i}\right) \cap \xi_{3} \neq \emptyset, e_{0} \in G\left(Y_{i}\right)$ and $Y_{0} \cong Y_{i}$, then $G\left(Y_{0}\right) \in\left\{\xi_{2}^{\prime} \cup\left\{e_{0}\right\}, \xi_{3}^{\prime} \cup\left\{e_{0}\right\}\right\}$ and one of Table $6[\mathrm{c}, \mathrm{d}]$ holds. We cannot apply Lemma 11a in this case.

- If $G\left(Y_{i}\right) \cap \xi_{3} \neq \emptyset, e_{0} \in G\left(Y_{i}\right)$ and $Y_{0} \not Y_{i}$, then $G\left(Y_{0}\right) \in\left\{\xi_{2}, \xi_{3}, \xi_{2} \cup \xi_{1}, \xi_{3} \cup \xi_{1}\right\}$ by Lemma 11a and one of Table $6[\mathrm{c}, \mathrm{d}]$ holds.

- If $G\left(Y_{i}\right) \cap \xi_{5} \neq \emptyset$, then $G\left(Y_{i}\right) \in\left\{\xi_{4}, \xi_{5}, \xi_{5} \cup\left\{\ell_{0}+\ell_{1}-\varepsilon_{1}\right\}\right\}$ by Lemma 11b and one of Table $6[\mathrm{~g}, \mathrm{~h}]$ holds.

See Example 3, Example 4 and Example 5 for possible scenarios. We describe two additional scenarios to clarify how the above observations are applied.

For example, if $G\left(Y_{\ell}\right)=\left\{e_{0}\right\}$ and $\mu_{\ell-1}$ contracts at least one real (-1)-curve, then $G\left(Y_{\ell-1}\right)=\xi_{1}$ and thus $G\left(Y_{0}\right)=\xi_{1}$ so that we are in case Table $6 \mathrm{~b}$ where $\alpha=\ell-1$. For another example, suppose that $G\left(Y_{\ell}\right)=\left\{\ell_{0}+\ell_{1}\right\}$ and $h_{\ell}=\ell_{0}+\ell_{1}$. If $\mu_{\ell-1}$ contracts exactly one real $(-1)$-curve, then $G\left(Y_{\ell-1}\right)=\left\{\ell_{0}+\ell_{1}-\varepsilon_{1}\right\}$. If $\mu_{\ell-2}$ contracts at least one real $(-1)$-curve, then $G\left(Y_{\ell-2}\right)=\xi_{4}$ so that we are in case Table $6 \mathrm{~g}$. If $\mu_{\ell-2}$ contracts only non-real complex $(-1)$-curves, then $h_{\ell}=5\left(\ell_{0}+\ell_{1}\right)-2 \varepsilon_{1}-\varepsilon_{2}-$ $\ldots-\varepsilon_{r}$ with $\sigma_{*}\left(\varepsilon_{i}\right) \neq \varepsilon_{i}$ for all $2 \leq i \leq r$ so that $G\left(Y_{\ell-2}\right)=\xi_{5} \cup\left\{\ell_{0}+\ell_{1}-\varepsilon_{1}\right\}$ and thus we are again in case Table $6 \mathrm{~g}$.

It is straightforward to verify that each scenario is characterized by one of the cases in Table 6 and that for each case in Table 6 there exists a scenario that realizes that case. 
Proof of Theorem 1. Suppose that $\left(Y_{0}, h_{0}\right) \stackrel{\mu_{0}}{\longrightarrow}\left(Y_{1}, h_{1}\right) \stackrel{\mu_{1}}{\longrightarrow} \ldots \stackrel{\mu_{\ell-1}}{\longrightarrow}\left(Y_{\ell}, h_{\ell}\right)$ is the unique adjoint chain of $X$. Recall that $G(X)=G\left(Y_{0}\right)$ and that we can recover $\mathcal{G}(X)$ from $G(X)$ (see Remark 3 and Lemma 10c). If $Y_{\ell}$ is not a weak del Pezzo surface, then we know from Lemma 9 that $|G(X)|=1$ so that $\mathcal{G}(X)$ is characterized by Theorem 1d. We suppose in the remainder of the proof that $Y_{\ell}$ is a weak del Pezzo surface and that $\mathcal{G}(X) \neq \emptyset$. Hence, one of the cases in Lemma 4 [a-g] holds. If $\left\{g \in G\left(Y_{\ell}\right) \mid k_{\ell} \cdot g=-2\right\} \neq \emptyset$, then $G\left(Y_{\ell}\right)=F_{\mathbb{R}}\left(Y_{\ell}\right)$ and thus, by Lemma 10b, $\mathcal{G}(X)$ is characterized by Theorem 1a. Now suppose that $\left\{g \in G\left(Y_{\ell}\right) \mid k_{\ell} \cdot g=-2\right\}=\emptyset$. It follows from Lemma 12 that $G\left(Y_{0}\right)$ is either $\left\{e_{0}\right\}, \xi_{1}, \xi_{2}, \xi_{2}^{\prime} \cup\left\{e_{0}\right\}, \xi_{2} \cup \xi_{1}, \xi_{3}$ $\xi_{3}^{\prime} \cup\left\{e_{0}\right\}, \xi_{3} \cup \xi_{1},\left\{\ell_{0}+\ell_{1}\right\},\left\{\ell_{0}+\ell_{1}-\varepsilon_{1}\right\}, \xi_{4}, \xi_{5} \cup\left\{\ell_{0}+\ell_{1}-\varepsilon_{1}\right\}$ or $\xi_{5}$ so that $\mathcal{G}(X)$ is characterized by (c), (d), (e), (k), (h), (f), (l), (i), (b), (c), (d), (k) and (g/j) in Theorem 1, respectively.

Proof of Corollary 1 and Corollary 2. Direct consequence of Theorem 2 (see also Table 4 and Remark 3). For the tedious calculations and checking each row of Table 7 for Corollary 2, we used [16, ns_lattice].

Proof of Corollary 3. Notice that Theorem 1b must hold and that $G\left(Y_{0}\right)=\left\{\ell_{0}+\ell_{1}\right\}$ so that $Y_{0} \in \mathbf{S}_{0}$ by Lemma 12. If $Y_{0} \in \mathbf{S}_{0}$, then $h_{0}=t \ell_{0}+t \ell_{1}$ for some $t \in \mathbb{Z}_{>0}$ and thus $Y_{0}$ is biregular isomorphic to a smooth non-ruled quadric. We concluded the proof as all such quadrics are projectively equivalent. Alternatively, see [18, Corollary 1a].

Proof of Theorem 4. Let $\varphi: Y \rightarrow X$ be the smooth model of $X \subset \mathbb{P}^{n}$ and let $h, k \in N(X)$ be the class of hyperplane sections and the canonical class, respectively. Recall from Remark 3 that two disjoint vertices correspond to simple families with classes $f, g \in G(X)$ such that $f \cdot g=1$. It follows from Theorem 1 that the vertices must have label 1 so that $h^{0}(f)=h^{0}(g)=2$. We know from Lemma 10c that $-k \cdot g=-2$ and since $p_{a}(f)=p_{a}(g)=0$ it follows from (AF) that $f^{2}=g^{2}=0$. Thus the fibers of the associated maps $\varphi_{f}: Y \rightarrow \mathbb{P}^{1}$ and $\varphi_{g}: Y \rightarrow \mathbb{P}^{1}$ are $\varphi$-preimages of simple curves on $X$ in their respective families. Let $\nu: Y \rightarrow \mathbb{P}^{1} \times \mathbb{P}^{1}$ be the morphism defined by $x \mapsto\left(\varphi_{f}(x), \varphi_{g}(x)\right)$. The map $\nu$ is birational, since the general fiber of $\varphi_{f}$ and $\varphi_{g}$ intersect in one point. Thus the inverse of $\nu$ composed with $\varphi$ defines a birational map $\mathbb{P}^{1} \times \mathbb{P}^{1} \rightarrow X$ of bidegree $(d, d)$, where $d$ is the degree of a simple curve on $X$. A birational map $\tau: \mathbb{P}^{1} \times \mathbb{P}^{1} \rightarrow X$ of bidegree $(a, b)$ defines two 
covering families of rational curves with members $\tau\left(\mathbb{P}^{1} \times \mathbb{P}^{1} \cap\{t\} \times \mathbb{P}^{1}\right)$ of degree $b$ and $\tau\left(\mathbb{P}^{1} \times \mathbb{P}^{1} \cap \mathbb{P}^{1} \times\{t\}\right)$ of degree $a$, respectively. This concludes the proof as $d$ is by definition the lowest possible degree for a rational curve in a covering family.

\section{A characterization of hexagonal webs}

In this section we prove Theorem 3.

Definition 6. Suppose that $X$ is a surface with real structure $\sigma$. Let $\mathcal{W}$ be a set of curves in $X$ and let $\mathcal{W}_{p}:=\{C \in \mathcal{W} \mid p \in C\}$ for all $p \in X$. Let $\mathcal{H}(\mathcal{W})$ be defined as the graph with vertex set $\left\{p \in X|| \mathcal{W}_{p} \mid=3\right.$ and $\left.\sigma(p)=p\right\}$ and labeled edge set

$$
\{(\{v, w\}, C) \mid C \in \mathcal{W} \text { and } v, w \in C \text { are pairwise distinct }\}
$$

We call $\mathcal{W}$ a hexagonal web if the vertex set of $\mathcal{H}(\mathcal{W})$ is not contained in a reducible curve and if a general edge $\{p, q\}$ of $\mathcal{H}(\mathcal{W})$ is contained in a subgraph as defined in Figure 4, where the edge-labels $A, B, C, D, E, F, G, H, I \in \mathcal{W}$ are pairwise distinct. $\triangleleft$

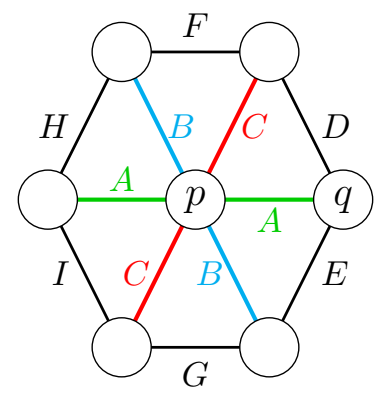

Figure 4: See Definition 6.

Remark 7. Suppose that $\mathcal{W}$ is a hexagonal web and that $\{p, q\}$ in Figure 5a corresponds to a general edge of $\mathcal{H}(\mathcal{W})$. In Figure $5 \mathrm{~b}$ we draw all the curves in $\mathcal{W}_{p} \cup \mathcal{W}_{q}$ and we obtain at least two new intersection points $r$ and $s$. In Figure $5 \mathrm{c}$ we draw all the curves in $\mathcal{W}_{r} \cup \mathcal{W}_{s}$ and we obtain again at least two new intersection points. We repeat the last step one more time so that we obtain a closed hexagon as in Figure 5d. Figure 5e is an example of a non-hexagonal web. We refer to [21] for more information. 


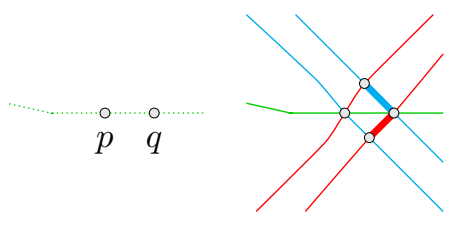

a

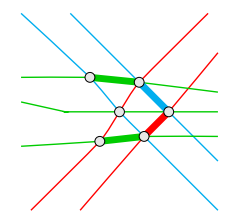

c

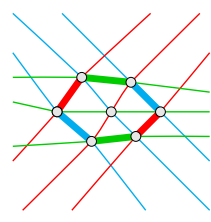

d

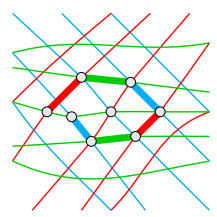

e

Figure 5: See Remark \%.

Definition 7. For the complex analogue of Definition 6 we suppose that $X$ is a complex surface. Let $\mathcal{W}$ be a set of complex curves in $X$ and as before let $\mathcal{W}_{p}:=$ $\{C \in \mathcal{W} \mid p \in C\}$ for all $p \in X$. Let $\mathcal{H}(\mathcal{W})$ be defined as the graph with vertex set $\left\{p \in X|| \mathcal{W}_{p} \mid=3\right\}$ and labeled edge set $\{(\{v, w\}, C) \mid C \in \mathcal{W}$ and $v, w \in$ $C$ are pairwise distinct $\}$. We call $\mathcal{W}$ a complex hexagonal web if the vertex set of $\mathcal{H}(\mathcal{W})$ is not contained in a complex reducible curve and if a general edge $\{p, q\}$ of $\mathcal{H}(\mathcal{W})$ is contained in a subgraph as defined in Figure 4, where the edge-labels $A, B, C, D, E, F, G, H, I \in \mathcal{W}$ are pairwise distinct.

Lemma 13. Suppose that $Y \rightarrow X$ is the smooth model of a weak del Pezzo surface $X$ such that $G(X)=F_{\mathbb{R}}(X)$. If there exists $u, v, w \in G(X)$ such that $u \cdot v=v \cdot w=$ $u \cdot w=1$, then there exists a complex birational morphism $\chi: Y \rightarrow \mathbb{P}^{2}$ such that images of simple curves with class $u, v$ or $w$ define three pencils of lines in $\mathbb{P}^{2}$.

Proof. Since $|G(X)| \geq 3$, we find that $1 \leq k^{2} \leq 6$ by Lemma 4 .

If $k^{2}=6$, then $Y$ is the complex blowup of $\mathbb{P}^{2}$ in three points and admits exactly three simple families. The pullback of a pencil of lines through any center of blowup defines a simple family and thus we concluded the proof for this case.

If $1 \leq k^{2} \leq 5$, then by Lemma 3c there exists a complex $(-1)$-curve that is orthogonal to $u, v$ and $w$. We contract it and by Lemma 2 we obtain a complex weak del Pezzo surface $Y^{\prime}$ of degree one less. Moreover, by Proposition 2c, the pushforward of the simple families with classes $u, v$ and $w$ are complex simple families of $Y^{\prime}$ with respect to an anticanonical embedding. We repeat the same argument until we obtain a complex weak del Pezzo surface $Y^{\prime \prime}$ of canonical degree 6 . We can now conclude the proof of this lemma, since we define $\xi_{\mathbb{C}}$ as the composition of the birational morphism $Y \rightarrow Y^{\prime \prime}$ with the complex birational morphism $Y^{\prime \prime} \rightarrow \mathbb{P}^{2}$ which is the complex blowup of three complex points in the plane. 
Lemma 14. Suppose we are given simple families on a surface $X$. If there exists a complex birational map $\rho: X \rightarrow \mathbb{P}^{2}$ such that the images of curves in the given simple families form three complex pencils of lines in $\mathbb{P}^{2}$, then these simple families form a hexagonal web.

Proof. Suppose that $\mathcal{L}:=\left\{L \subset \mathbb{P}^{2} \mid L\right.$ is a complex line such that $\left.L \cap\{u, v, w\} \neq \emptyset\right\}$ for some complex points $u, v, w \in \mathbb{P}^{2}$. It follows from Theorem $\mathrm{A}$ that $\mathcal{L}$ is a complex hexagonal web, since three complex pencils of lines in $\mathbb{P}^{2}$ correspond to three complex lines in $\mathbb{P}^{2 *}$ (see Figure 3). The union of the members of the simple families form a set $\mathcal{V}$ such that $\left\{\rho(C)^{-} \mid C \in \mathcal{V}\right\} \subseteq \mathcal{L}$, where $\cdot{ }^{-}$denotes the Zariski closure. Since $\rho$ is birational there exists a Zariski open subset $U \subset X$ such that $\left.\rho\right|_{U}$ is a complex isomorphism. We follow the procedure in Remark 7 and when we draw distinct simple curves $C, C^{\prime} \in \mathcal{V}$ we also draw complex lines $\rho(C)^{-}, \rho\left(C^{\prime}\right)^{-} \in \mathcal{L}$. We have $\left|\rho\left(C \cap C^{\prime} \cap U\right)\right| \leq 1$ and thus if $\left|C \cap C^{\prime} \cap U\right| \neq 0$, then $C \cap C^{\prime} \cap U=\{p\}$ and $\sigma(p)=p$. Hence there exists in this case a simple curve $C^{\prime \prime} \in \mathcal{V}$ such that $p \in C^{\prime \prime}$ and $C^{\prime \prime} \notin\left\{C, C^{\prime}\right\}$. Therefore the procedure results in a closed hexagon so that $\mathcal{V}$ must be a hexagonal web.

Proof of Theorem 3. Suppose that $\left(Y_{0}, h_{0}\right) \stackrel{\mu_{0}}{\longrightarrow}\left(Y_{1}, h_{1}\right) \stackrel{\mu_{1}}{\longrightarrow} \ldots \stackrel{\mu_{\ell-1}}{\longrightarrow}\left(Y_{\ell}, h_{\ell}\right)$ is the adjoint chain of $X$. Recall that $G\left(Y_{0}\right)$ uniquely determines $\mathcal{G}(X)$ (see Remark 3 and Lemma 10c). It follows from Lemma 9 that $Y_{\ell}$ must be a weak del Pezzo surface. We call a curve $C \subset Y_{i}$ on the abstract surface $Y_{i}$ simple if its class is in $G\left(Y_{i}\right)$. Since $Y_{0} \rightarrow X$ is the smooth model, there exists a birational map $\varphi: X \rightarrow Y_{0}$ which sends simple curves on $X$ to simple curves on $Y_{0}$.

We will make a case distinction. For each of the cases we construct a complex birational map $X \rightarrow \mathbb{P}^{2}$ that sends the curves in the three simple families of $X$, that define three mutually disjoint vertices in $\mathcal{G}(X)$, to three pencils of lines in $\mathbb{P}^{2}$. Such a map satisfies the hypothesis of Lemma 14 so that the simple families form a hexagonal web and therefore its existence concludes the proof.

Suppose that $\left\{f \in G\left(Y_{\ell}\right) \mid k_{\ell} \cdot f=-2\right\} \neq \emptyset$ so that we are in case Lemma 9a. It follows from Lemma 4 that $G\left(Y_{\ell}\right)=F_{\mathbb{R}}\left(Y_{\ell}\right)$ and thus $\mathcal{G}(X)$ is characterized by Theorem 1a. Notice that there exists classes $u, v, w \in F_{\mathbb{R}}\left(Y_{\ell}\right)$ such that $u \cdot v=$ $u \cdot w=v \cdot w=1$. By Lemma 13 there exists a complex birational morphism $\chi: Y_{\ell} \rightarrow \mathbb{P}^{2}$ that sends a simple curve with class $u, v$ or $w$ to one of three pencils 
of lines in $\mathbb{P}^{2}$. As a straightforward consequence of the definitions, the birational morphism $\mu_{\ell-1} \circ \ldots \circ \mu_{0}$ maps simple curves of $Y_{0}$ to simple curves of $Y_{\ell}$. Hence, the composition $\chi \circ \mu_{\ell-1} \circ \ldots \circ \mu_{0} \circ \varphi: X \rightarrow \mathbb{P}^{2}$ satisfies the hypothesis of Lemma 14 . Now suppose that $\left\{f \in G\left(Y_{\ell}\right) \mid k_{\ell} \cdot f=-2\right\}=\emptyset$. Notice that there exists $u, v, w \in$ $G\left(Y_{0}\right)$ such that $u \cdot v=u \cdot w=v \cdot w=1$. It follows from Lemma 12 that one of the cases at Table $6[\mathrm{a}, \mathrm{c}, \mathrm{d}, \mathrm{g}, \mathrm{h}]$ holds and $\{u, v, w\}$ is a subset of either $\xi_{1}, \xi_{4}$ or $\xi_{5}$. We use the notation of Table 6 .

If Table 6a holds, then the birational map $\mu_{\ell-1} \circ \ldots \circ \mu_{0} \circ \varphi: X \rightarrow \mathbb{P}^{2}$ satisfies the hypothesis of Lemma 14.

Suppose that Table $6 \mathrm{c}$ holds. Notice that in this case $\beta>\alpha$. We may assume up to permutation of $\left(e_{j}\right)_{j>0}$ that $(u, v, w)=\left(e_{0}-e_{7}, e_{0}-e_{8}, e_{0}-e_{9}\right)$ (we chose indices 7,8 and 9 in accordance with Example 4). It follows from Lemma $9 \mathrm{~b}$ that there exists a birational map $\eta: Y_{\beta} \rightarrow \mathbb{P}^{2}$ that sends the curves $\left\{C \subset Y_{\beta} \mid[C] \in\left\{e_{0}-e_{7}, e_{0}-\right.\right.$ $\left.\left.e_{8}, e_{0}-e_{9}\right\}\right\}$ to three pencils of lines in $\mathbb{P}^{2}$. It follows that $\eta \circ \mu_{\beta-1} \circ \ldots \circ \mu_{0} \circ \varphi: X \rightarrow \mathbb{P}^{2}$ satisfies the hypothesis of Lemma 14.

Suppose that Table $6 \mathrm{~g}$ holds such that $Y_{\alpha} \in \mathbf{S}_{1}$. We may assume up to permutation of $\left(\varepsilon_{j}\right)_{j>0}$ that $(u, v, w)=\left(\ell_{0}+\ell_{1}-\varepsilon_{1}-\varepsilon_{2}, \ell_{0}+\ell_{1}-\varepsilon_{1}-\varepsilon_{3}, \ell_{0}+\ell_{1}-\varepsilon_{1}-\varepsilon_{4}\right)$. Notice that $\mathbb{P}^{1} \times \mathbb{P}^{1}$ blown up in one point is complex isomorphic to $\mathbb{P}^{2}$ blownup in two points. Thus there exists a complex isomorphism $\psi_{1}: Y_{\alpha} \rightarrow Z$ such that $Z \in \mathbf{P}_{1}$ and such that $\psi_{1 *}\left(\ell_{0}+\ell_{1}-\varepsilon_{1}\right)=e_{0}, \psi_{1 *}\left(\ell_{0}-\varepsilon_{1}\right)=e_{1}, \psi_{1 *}\left(\ell_{1}-\varepsilon_{1}\right)=e_{2}$ and $\psi_{1 *}\left(\varepsilon_{j}\right)=e_{j+1}$ for $j \geq 2$. Thus $\psi_{1 *}(\{u, v, w\})=\left\{e_{0}-e_{3}, e_{0}-e_{4}, e_{0}-e_{5}\right\}$ where $e_{0}$ is the class of a preimage of a line along a birational morphism $\psi_{2}: Z \rightarrow \mathbb{P}^{2}$. It follows that the complex birational map $\psi_{2} \circ \psi_{1} \circ \mu_{\alpha-1} \circ \ldots \circ \mu_{0} \circ \varphi: X \rightarrow \mathbb{P}^{2}$ satisfies the hypothesis of Lemma 14 .

Cases Table $6 \mathrm{~d}$ and Table $6 \mathrm{~h}$ are analogous to Table $6 \mathrm{c}$ and Table $6 \mathrm{~g}$, respectively. Since we considered all cases we concluded the proof of Theorem 3. 
Proof of Corollary 4. By assumption, $X \subset \mathbb{P}^{n}$ contains at least two conics through each point, and thus we know from [27] (alternatively see [18, Corollary 2]), that $X$ is either a ruled surface of degree at most 3 or a weak del Pezzo surface of canonical degree $3 \leq k^{2} \leq 9$ with $h \in\left\{-\frac{1}{2} k,-\frac{1}{3} k,-\frac{2}{3} k,-k\right\}$ the class of hyperplane sections. The linear projection of a hexagonal web is again hexagonal and thus we only have to classify linear normalizations $X_{N} \subset \mathbb{P}^{m}$.

If $X$ is a cubic ruled surface, then $X_{N} \subset \mathbb{P}^{4}$ is parametrized by the map $\left(1: t: t^{2}\right.$ : $s: s t)$ with $t, s \in \mathbb{R}[27$, Section 3]. This birational map gives, for all $\beta \in \mathbb{R}$, locally an isomorphism between a pencil of conics on $X_{N}$ and a pencil of lines in $\mathbb{R}^{2}$ defined by $s=\alpha t+\beta$ with parameter $\alpha \in \mathbb{R}$. Therefore, by Theorem A, $X$ can be covered by an hexagonal web of conics.

Suppose that $X$ is a weak del Pezzo surface. If $8 \leq k^{2} \leq 9$, then $X_{N}$ must be either a quadric surface, the plane or a Veronese surface, since $X$ contains at least three conics through a general point. Each of these surfaces can be realized as a hexagonal web (see Figure 3). If $3 \leq k^{2} \leq 6$, then $h=-k, X_{N} \subset \mathbb{P}^{k^{2}}$ and by Theorem 2 we can construct a weak del Pezzo surface of degree $k^{2}$ such that $|G(X)| \geq 3$ and $\mathcal{G}(X)$ contains three vertices that do not share an edge. Thus Corollary $4 \mathrm{e}$ is a consequence of Theorem 3. 
Table 7: Classification of Neron-Severi lattices of weak del Pezzo surfaces (see Theorem 2).

- Each row in the table below after the dictionary (except for the 5 rows with the $\times$-symbol) encodes the Neron-Severi lattice $\mathbf{N}(X)$ of a weak del Pezzo surface (see Definition 1).

- The columns $d, D(A), D(B), \# E$, \#G stand for $k^{2}, \mathfrak{D}(A(X)), \mathfrak{D}(B(X))$, $\left|E_{\mathbb{R}}(X)\right|$ and $|G(X)|$, respectively. The entries of the columns $\mathfrak{D}(A(X))$ and $\mathfrak{D}(B(X))$ denote Dynkin types of the corresponding Dynkin diagrams.

- See Table 1, Proposition 2 and Proposition 3 for the interpretation of the columns. It follows from Lemma 4 that $G(X)=F_{\mathbb{R}}(X)$ except for the rows i, iii and 2. By Remark 3, $G(X)$ uniquely determines the simple family graph $\mathcal{G}(X)$.

- There is not always a unique choice for the Dynkin type at column $D(A)$ (see Remark 6). We decorate a Dynkin type with ' if $\sigma_{*}\left(e_{0}\right) \neq e_{0}$.

- For each component $W \subset B(X)$ (see Definition 5) the Dynkin type of $\mathfrak{D}(W)$ at column $D(B)$ is solid underlined if $\sigma_{*}(w)=w$ for all $w \in W$ and dashed underlined if $\sigma_{*}(W)=W$ but there exists $w \in W$ such that $\sigma_{*}(w) \neq w$. If $W, W^{\prime} \subset B(X)$ are different components such that $\sigma_{*}(W)=W^{\prime}$, then the corresponding Dynkin types are not decorated.

- From the entry at column $\sigma_{A} \| B$ we can recover $\sigma_{*}: N(X) \rightarrow N(X)$ and $B(X)$ using the dictionary below. For example, at row number 10, the entry $\frac{0}{0} \frac{0}{2} \frac{0}{1} \frac{0}{3} \| \frac{3}{7}$ encodes that $\sigma_{*}\left(e_{0}\right)=e_{0}, \sigma_{*}\left(e_{1}\right)=e_{2}, \sigma_{*}\left(e_{2}\right)=e_{1}, \sigma_{*}\left(e_{3}\right)=e_{3}$ and $B(X)=$ $\left\{e_{0}-e_{1}-e_{2}-e_{3}\right\}$ with respect to a type 1 basis $\left\langle e_{0}, \ldots, e_{r}\right\rangle_{\mathbb{Z}}$ for $N(X)$ (see Definition 2). It follows from Remark 4 that $A(X)=\left\{e_{1}-e_{2}\right\}$. Indeed we verify that $\mathfrak{D}(A(X))=A_{1}$ and $B(X)=\underline{A_{1}}$.

- We can uniquely recover $h^{0}: N(X) \rightarrow \mathbb{Z}_{\geq 0}$ from $B(X)$ (see Lemma 5) and thus the entries of the columns in a row accounts for all data of $\mathbf{N}(X)$.

- See Example 2 for a scenario which explains how the table can be used. 
A dictionary for symbols in the column $\sigma_{A} \| B$ :

\begin{tabular}{|c|c|}
\hline$\frac{0}{0}: e_{0}$ & $\frac{5}{0}: e_{0}-e_{1}-e_{3}-e_{6}$ \\
\hline$\frac{0}{1}: e_{1}$ & $\frac{5}{1}: e_{0}-e_{2}-e_{3}-e_{6}$ \\
\hline$\frac{0}{2}: e_{2}$ & $\frac{5}{2}: e_{0}-e_{1}-e_{4}-e_{6}$ \\
\hline$\frac{0}{3}: e_{3}$ & $\frac{5}{3}: e_{0}-e_{2}-e_{4}-e_{6}$ \\
\hline$\frac{0}{4}: e_{4}$ & $\frac{5}{4}: e_{0}-e_{3}-e_{4}-e_{6}$ \\
\hline$\frac{0}{5}: e_{5}$ & $\frac{5}{5}: e_{0}-e_{1}-e_{5}-e_{6}$ \\
\hline$\frac{0}{6}: e_{6}$ & $\frac{5}{6}: e_{0}-e_{3}-e_{5}-e_{6}$ \\
\hline$\frac{0}{7}: e_{7}$ & $\frac{5}{7}: e_{0}-e_{4}-e_{5}-e_{6}$ \\
\hline$\frac{0}{8}: e_{8}$ & $\frac{5}{8}: e_{0}-e_{1}-e_{7}$ \\
\hline$\frac{0}{9}: e_{1}-e_{2}$ & $\frac{5}{9}: e_{0}-e_{1}-e_{2}-e_{7}$ \\
\hline$\frac{1}{0}: e_{2}-e_{3}$ & $\frac{6}{0}: e_{0}-e_{1}-e_{3}-e_{7}$ \\
\hline$\frac{1}{1}: e_{3}-e_{4}$ & $\frac{6}{1}: e_{0}-e_{2}-e_{3}-e_{7}$ \\
\hline$\frac{1}{2}: e_{2}-e_{4}$ & $\frac{6}{2}: e_{0}-e_{1}-e_{4}-e_{7}$ \\
\hline$\frac{1}{3}: e_{1}-e_{4}$ & $\frac{6}{3}: e_{0}-e_{2}-e_{4}-e_{7}$ \\
\hline$\frac{1}{4}: e_{4}-e_{5}$ & $\frac{6}{4}: e_{0}-e_{3}-e_{4}-e_{7}$ \\
\hline$\frac{1}{5}: e_{3}-e_{5}$ & $\frac{6}{5}: e_{0}-e_{1}-e_{5}-e_{7}$ \\
\hline$\frac{1}{6}: e_{2}-e_{5}$ & $\frac{6}{6}: e_{0}-e_{2}-e_{5}-e_{7}$ \\
\hline$\frac{1}{7}: e_{5}-e_{6}$ & $\frac{6}{7}: e_{0}-e_{3}-e_{5}-e_{7}$ \\
\hline$\frac{1}{8}: e_{4}-e_{6}$ & $\frac{6}{8}: e_{0}-e_{4}-e_{5}-e_{7}$ \\
\hline$\frac{1}{9}: e_{3}-e_{6}$ & $\frac{6}{9}: e_{0}-e_{1}-e_{6}-e_{7}$ \\
\hline$\frac{2}{0}: e_{2}-e_{6}$ & $\frac{7}{0}: e_{0}-e_{2}-e_{6}-e_{7}$ \\
\hline$\frac{2}{1}: e_{1}-e_{6}$ & $\frac{7}{1}: e_{0}-e_{3}-e_{6}-e_{7}$ \\
\hline$\frac{2}{2}: e_{6}-e_{7}$ & $\frac{7}{2}: e_{0}-e_{4}-e_{6}-e_{7}$ \\
\hline$\frac{2}{3}: e_{5}-e_{7}$ & $\frac{7}{3}: e_{0}-e_{5}-e_{6}-e_{7}$ \\
\hline$\frac{2}{4}: e_{4}-e_{7}$ & $\frac{7}{4}: e_{0}-e_{1}-e_{2}-e_{8}$ \\
\hline$\frac{2}{5}: e_{3}-e_{7}$ & $\frac{7}{5}: e_{0}-e_{1}-e_{3}-e_{8}$ \\
\hline$\frac{2}{6}: e_{2}-e_{7}$ & $\frac{7}{6}: e_{0}-e_{2}-e_{3}-e_{8}$ \\
\hline$\frac{2}{7}: e_{1}-e_{7}$ & $\frac{7}{7}: e_{0}-e_{1}-e_{4}-e_{8}$ \\
\hline$\frac{2}{8}: e_{7}-e_{8}$ & $\frac{7}{8}: e_{0}-e_{2}-e_{4}-e_{8}$ \\
\hline$\frac{2}{9}: e_{6}-e_{8}$ & $\frac{7}{9}: e_{0}-e_{3}-e_{4}-e_{8}$ \\
\hline$\frac{3}{0}: e_{5}-e_{8}$ & $\frac{8}{0}: e_{0}-e_{1}-e_{5}-e_{8}$ \\
\hline$\frac{3}{1}: e_{4}-e_{8}$ & $\frac{8}{1}: e_{0}-e_{2}-e_{5}-e_{8}$ \\
\hline$\frac{3}{2}: e_{3}-e_{8}$ & $\frac{8}{2}: e_{0}-e_{3}-e_{5}-e_{8}$ \\
\hline$\frac{3}{3}: e_{1}-e_{8}$ & $\frac{8}{3}: e_{0}-e_{4}-e_{5}-e_{8}$ \\
\hline$\frac{3}{4}: e_{0}-e_{1}-e_{2}$ & $\frac{8}{4}: e_{0}-e_{1}-e_{6}-e_{8}$ \\
\hline$\frac{3}{5}: e_{0}-e_{1}-e_{3}$ & $\frac{8}{5}: e_{0}-e_{2}-e_{6}-e_{8}$ \\
\hline$\frac{3}{6}: e_{0}-e_{2}-e_{3}$ & $\frac{8}{6}: e_{0}-e_{3}-e_{6}-e_{8}$ \\
\hline$\frac{3}{7}: e_{0}-e_{1}-e_{2}-e_{3}$ & $\frac{8}{7}: e_{0}-e_{4}-e_{6}-e_{8}$ \\
\hline$\frac{3}{8}: e_{0}-e_{1}-e_{4}$ & $\frac{8}{8}: e_{0}-e_{5}-e_{6}-e_{8}$ \\
\hline$\frac{3}{9}: e_{0}-e_{1}-e_{3}-e_{4}$ & $\frac{8}{9}: e_{0}-e_{1}-e_{7}-e_{8}$ \\
\hline$\frac{4}{0}: e_{0}-e_{2}-e_{3}-e_{4}$ & $\frac{9}{0}: e_{0}-e_{2}-e_{7}-e_{8}$ \\
\hline$\frac{4}{1}: e_{0}-e_{1}-e_{5}$ & $\frac{9}{1}: e_{0}-e_{3}-e_{7}-e_{8}$ \\
\hline$\frac{4}{2}: e_{0}-e_{1}-e_{2}-e_{5}$ & $\frac{9}{2}: e_{0}-e_{4}-e_{7}-e_{8}$ \\
\hline$\frac{4}{3}: e_{0}-e_{1}-e_{3}-e_{5}$ & $\frac{9}{3}: e_{0}-e_{5}-e_{7}-e_{8}$ \\
\hline$\frac{4}{4}: e_{0}-e_{2}-e_{3}-e_{5}$ & $\frac{9}{4}: e_{0}-e_{6}-e_{7}-e_{8}$ \\
\hline$\frac{4}{5}: e_{0}-e_{1}-e_{4}-e_{5}$ & $\frac{9}{5}: 2 e_{0}-e_{1}-e_{2}-e_{3}$ \\
\hline$\frac{4}{6}: e_{0}-e_{2}-e_{4}-e_{5}$ & $\frac{9}{6}: 2 e_{0}-e_{1}-e_{2}-e_{3}-e_{4}-e_{5}$ \\
\hline$\frac{4}{7}: e_{0}-e_{3}-e_{4}-e_{5}$ & $\frac{9}{7}: 2 e_{0}-e_{1}-e_{2}-e_{3}-e_{4}-e_{5}-e_{6}$ \\
\hline$\frac{4}{8}: e_{0}-e_{1}-e_{6}$ & $\frac{9}{8}: 2 e_{0}-e_{1}-e_{2}-e_{3}-e_{4}-e_{5}-e_{7}$ \\
\hline$\frac{4}{9}: e_{0}-e_{1}-e_{2}-e_{6}$ & $\frac{9}{9}: 2 e_{0}-e_{1}-e_{2}-e_{3}-e_{4}-e_{6}-e_{7}$ \\
\hline
\end{tabular}

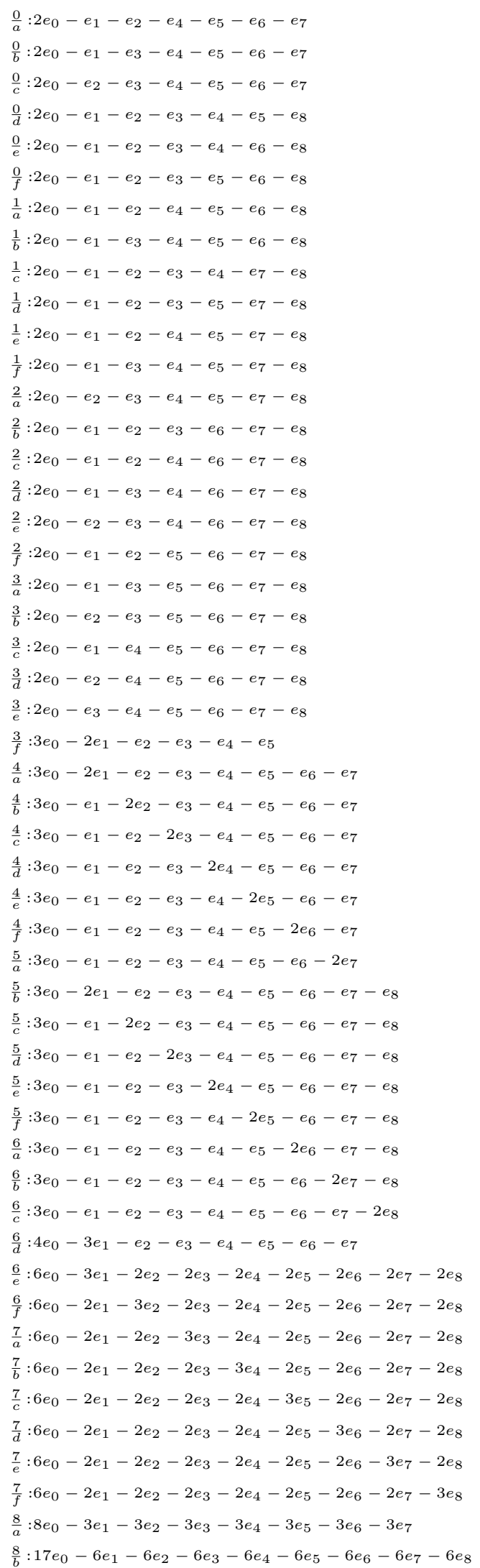




\begin{tabular}{|c|c|c|c|c|c|c|}
\hline & $\mathrm{d}$ & $D(A)$ & $D(B)$ & $\# E$ & $\# G$ & $\sigma_{A} \| B$ \\
\hline i & 9 & $A_{0}$ & $A_{0}$ & 0 & 1 & \\
\hline ii & 8 & $A_{0}$ & $A_{0}$ & 0 & 2 & \\
\hline iii & 8 & $A_{0}$ & $A_{0}$ & 0 & 1 & \\
\hline iv & 8 & $A_{0}$ & $A_{0}$ & 1 & 1 & \\
\hline $\mathrm{v}$ & 8 & $A_{0}$ & $A_{1}$ & 0 & 1 & \\
\hline 0 & 7 & $A_{0}$ & $A_{0}$ & 3 & 2 & $\frac{0}{0} \frac{0}{1} \frac{0}{2} \|$ \\
\hline 1 & 7 & $A_{0}$ & $\underline{A_{1}}$ & 2 & 1 & $\frac{0}{0} \frac{0}{1} \frac{0}{2} \| \frac{0}{9}$ \\
\hline 2 & 7 & $A_{1}$ & $A_{0}$ & 1 & 1 & $\frac{0}{0} \frac{0}{2} \frac{0}{1} \|$ \\
\hline 3 & 6 & $A_{0}$ & $A_{0}$ & 6 & 3 & $\frac{0}{0} \frac{0}{1} \frac{0}{2} \frac{0}{3} \|$ \\
\hline 4 & 6 & $A_{0}$ & $\underline{A_{1}}$ & 4 & 2 & $\frac{0}{0} \frac{0}{1} \frac{0}{2} \frac{0}{3} \| \frac{0}{9}$ \\
\hline 5 & 6 & $A_{0}$ & $\underline{A_{1}}$ & 3 & 3 & $\frac{0}{0} \frac{0}{1} \frac{0}{2} \frac{0}{3} \| \frac{3}{7}$ \\
\hline 6 & 6 & $A_{0}$ & $2 \underline{A_{1}}$ & 2 & 2 & $\frac{0}{0} \frac{0}{1} \frac{0}{2} \frac{0}{3} \| \frac{0}{9} \frac{3}{7}$ \\
\hline 7 & 6 & $A_{0}$ & $\underline{A_{2}}$ & 2 & 1 & $\frac{0}{0} \frac{0}{1} \frac{0}{2} \frac{0}{3} \| \frac{0}{9} \frac{1}{0}$ \\
\hline 8 & 6 & $A_{0}$ & $\underline{A_{1}}+\underline{A_{2}}$ & 1 & 1 & $\frac{0}{0} \frac{0}{1} \frac{0}{2} \frac{0}{3} \| \frac{0}{9} \frac{1}{0} \frac{3}{7}$ \\
\hline 9 & 6 & $A_{1}$ & $A_{0}$ & 2 & 1 & $\frac{0}{0} \frac{0}{2} \frac{0}{1} \frac{0}{3} \|$ \\
\hline 10 & 6 & $A_{1}$ & $\underline{A_{1}}$ & 1 & 1 & $\frac{0}{0} \frac{0}{2} \frac{0}{1} \frac{0}{3} \| \frac{3}{7}$ \\
\hline 11 & 6 & $A_{1}^{\prime}$ & $A_{0}$ & 0 & 3 & $\frac{9}{5} \frac{3}{6} \frac{3}{5} \frac{3}{4} \|$ \\
\hline 12 & 6 & $A_{1}^{\prime}$ & $\underline{A_{1}}$ & 0 & 2 & $\frac{9}{5} \frac{3}{6} \frac{3}{5} \frac{3}{4} \| \frac{0}{9}$ \\
\hline 13 & 6 & $A_{1}^{\prime}$ & $\underline{A_{2}}$ & 0 & 1 & $\frac{9}{5} \frac{3}{6} \frac{3}{5} \frac{3}{4} \| \frac{0}{9} \frac{1}{0}$ \\
\hline 14 & 6 & $2 A_{1}$ & $A_{0}$ & 0 & 1 & $\frac{9}{5} \frac{3}{5} \frac{3}{6} \frac{3}{4} \|$ \\
\hline 15 & 5 & $A_{0}$ & $A_{0}$ & 10 & 5 & $\frac{0}{0} \frac{0}{1} \frac{0}{2} \frac{0}{3} \frac{0}{4} \|$ \\
\hline 16 & 5 & $A_{0}$ & $\underline{A_{1}}$ & 7 & 4 & $\frac{0}{0} \frac{0}{1} \frac{0}{2} \frac{0}{3} \frac{0}{4} \| \frac{0}{9}$ \\
\hline 17 & 5 & $A_{0}$ & $2 \underline{A_{1}}$ & 5 & 3 & $\frac{0}{0} \frac{0}{1} \frac{0}{2} \frac{0}{3} \frac{0}{4} \| \frac{0}{9} \frac{1}{1}$ \\
\hline 18 & 5 & $A_{0}$ & $\underline{A_{2}}$ & 4 & 3 & $\frac{0}{0} \frac{0}{1} \frac{0}{2} \frac{0}{3} \frac{0}{4} \| \frac{0}{9} \frac{1}{0}$ \\
\hline 19 & 5 & $A_{0}$ & $\underline{A_{1}}+\underline{A_{2}}$ & 3 & 2 & $\frac{0}{0} \frac{0}{1} \frac{0}{2} \frac{0}{3} \frac{0}{4} \| \frac{0}{9} \frac{1}{0} \frac{3}{7}$ \\
\hline 20 & 5 & $A_{0}$ & $\underline{A_{3}}$ & 2 & 2 & $\frac{0}{0} \frac{0}{1} \frac{0}{2} \frac{0}{3} \frac{0}{4} \| \frac{0}{9} \frac{1}{0} \frac{1}{1}$ \\
\hline 21 & 5 & $A_{0}$ & $\underline{A_{4}}$ & 1 & 1 & $\frac{0}{0} \frac{0}{1} \frac{0}{2} \frac{0}{3} \frac{0}{4} \| \frac{0}{9} \frac{1}{0} \frac{1}{1} \frac{3}{7}$ \\
\hline 22 & 5 & $A_{1}$ & $A_{0}$ & 4 & 3 & $\frac{0}{0} \frac{0}{2} \frac{0}{1} \frac{0}{3} \frac{0}{4} \|$ \\
\hline 23 & 5 & $A_{1}$ & $\underline{A_{1}}$ & 3 & 2 & $\frac{0}{0} \frac{0}{2} \frac{0}{1} \frac{0}{3} \frac{0}{4} \| \frac{1}{1}$ \\
\hline 24 & 5 & $A_{1}$ & $\underline{A_{2}}$ & 2 & 1 & $\frac{0}{0} \frac{0}{2} \frac{0}{1} \frac{0}{3} \frac{0}{4} \| \frac{3}{7} \frac{1}{1}$ \\
\hline 25 & 5 & $2 A_{1}$ & $A_{0}$ & 2 & 1 & $\frac{0}{0} \frac{0}{2} \frac{0}{1} \frac{0}{4} \frac{0}{3} \|$ \\
\hline 26 & 5 & $2 A_{1}$ & $2 A_{1}$ & 1 & 1 & $\frac{0}{0} \frac{0}{2} \frac{0}{1} \frac{0}{4} \frac{0}{3} \| \frac{1}{0} \frac{1}{3}$ \\
\hline
\end{tabular}

\begin{tabular}{|c|c|c|c|c|c|c|}
\hline & $\mathrm{d}$ & $D(A)$ & $D(B)$ & $\# E$ & $\# G$ & $\sigma_{A} \| B$ \\
\hline 27 & 4 & $A_{0}$ & $A_{0}$ & 16 & 10 & $\frac{0}{0} \frac{0}{1} \frac{0}{2} \frac{0}{3} \frac{0}{4} \frac{0}{5} \|$ \\
\hline 28 & 4 & $A_{0}$ & $\underline{A_{1}}$ & 12 & 8 & $\frac{0}{0} \frac{0}{1} \frac{0}{2} \frac{0}{3} \frac{0}{4} \frac{0}{5} \| \frac{0}{9}$ \\
\hline 29 & 4 & $A_{0}$ & $2 \underline{A_{1}}$ & 9 & 6 & $\frac{0}{0} \frac{0}{1} \frac{0}{2} \frac{0}{3} \frac{0}{4} \frac{0}{5} \| \frac{0}{9} \frac{1}{1}$ \\
\hline 30 & 4 & $A_{0}$ & $2 \underline{A_{1}}$ & 8 & 7 & $\frac{0}{0} \frac{0}{1} \frac{0}{2} \frac{0}{3} \frac{0}{4} \frac{0}{5} \| \frac{1}{4} \frac{3}{7}$ \\
\hline 31 & 4 & $A_{0}$ & $\underline{A_{2}}$ & 8 & 6 & $\frac{0}{0} \frac{0}{1} \frac{0}{2} \frac{0}{3} \frac{0}{4} \frac{0}{5} \| \frac{0}{9} \frac{1}{0}$ \\
\hline 32 & 4 & $A_{0}$ & $3 \underline{A_{1}}$ & 6 & 5 & $\frac{0}{0} \frac{0}{1} \frac{0}{2} \frac{0}{3} \frac{0}{4} \frac{0}{5} \| \frac{0}{9} \frac{1}{4} \frac{3}{7}$ \\
\hline 33 & 4 & $A_{0}$ & $\underline{A_{1}}+\underline{A_{2}}$ & 6 & 4 & $\frac{0}{0} \frac{0}{1} \frac{0}{2} \frac{0}{3} \frac{0}{4} \frac{0}{5} \| \frac{0}{9} \frac{1}{0} \frac{1}{4}$ \\
\hline 34 & 4 & $A_{0}$ & $\underline{A_{3}}$ & 5 & 4 & $\frac{0}{0} \frac{0}{1} \frac{0}{2} \frac{0}{3} \frac{0}{4} \frac{0}{5} \| \frac{0}{9} \frac{1}{0} \frac{1}{1}$ \\
\hline 35 & 4 & $A_{0}$ & $\underline{A_{3}}$ & 4 & 5 & $\frac{0}{0} \frac{0}{1} \frac{0}{2} \frac{0}{3} \frac{0}{4} \frac{0}{5} \| \frac{1}{1} \frac{1}{4} \frac{3}{7}$ \\
\hline 36 & 4 & $A_{0}$ & $2 \underline{A_{1}}+\underline{A_{2}}$ & 4 & 3 & $\frac{0}{0} \frac{0}{1} \frac{0}{2} \frac{0}{3} \frac{0}{4} \frac{0}{5} \| \frac{0}{9} \frac{1}{0} \frac{1}{4} \frac{3}{7}$ \\
\hline 37 & 4 & $A_{0}$ & $4 \underline{A_{1}}$ & 4 & 4 & $\frac{0}{0} \frac{0}{1} \frac{0}{2} \frac{0}{3} \frac{0}{4} \frac{0}{5} \| \frac{1}{0} \frac{1}{4} \frac{3}{7} \frac{4}{5}$ \\
\hline 38 & 4 & $A_{0}$ & $\underline{A_{1}}+\underline{A_{3}}$ & 3 & 3 & $\frac{0}{0} \frac{0}{1} \frac{0}{2} \frac{0}{3} \frac{0}{4} \frac{0}{5} \| \frac{0}{9} \frac{1}{1} \frac{1}{4} \frac{3}{7}$ \\
\hline 39 & 4 & $A_{0}$ & $\underline{A_{4}}$ & 3 & 2 & $\frac{0}{0} \frac{0}{1} \frac{0}{2} \frac{0}{3} \frac{0}{4} \frac{0}{5} \| \frac{0}{9} \frac{1}{0} \frac{1}{1} \frac{1}{4}$ \\
\hline 40 & 4 & $A_{0}$ & $\underline{D_{4}}$ & 2 & 3 & $\frac{0}{0} \frac{0}{1} \frac{0}{2} \frac{0}{3} \frac{0}{4} \frac{0}{5} \| \frac{1}{0} \frac{1}{1} \frac{1}{4} \frac{3}{7}$ \\
\hline 41 & 4 & $A_{0}$ & $2 \underline{A_{1}}+\underline{A_{3}}$ & 2 & 2 & $\frac{0}{0} \frac{0}{1} \frac{0}{2} \frac{0}{3} \frac{0}{4} \frac{0}{5} \| \frac{0}{9} \frac{1}{0} \frac{1}{4} \frac{3}{7} \frac{4}{5}$ \\
\hline 42 & 4 & $A_{0}$ & $\underline{D_{5}}$ & 1 & 1 & $\frac{0}{0} \frac{0}{1} \frac{0}{2} \frac{0}{3} \frac{0}{4} \frac{0}{5} \| \frac{0}{9} \frac{1}{0} \frac{1}{1} \frac{1}{4} \frac{3}{7}$ \\
\hline 43 & 4 & $A_{1}$ & $A_{0}$ & 8 & 6 & $\frac{0}{0} \frac{0}{2} \frac{0}{1} \frac{0}{3} \frac{0}{4} \frac{0}{5} \|$ \\
\hline 44 & 4 & $A_{1}$ & $\underline{A_{1}}$ & 6 & 4 & $\frac{0}{0} \frac{0}{2} \frac{0}{1} \frac{0}{3} \frac{0}{4} \frac{0}{5} \| \frac{1}{4}$ \\
\hline 45 & 4 & $A_{1}$ & $\underline{A_{1}}$ & 4 & 6 & $\frac{0}{0} \frac{0}{2} \frac{0}{1} \frac{0}{3} \frac{0}{4} \frac{0}{5} \| \frac{4}{7}$ \\
\hline 46 & 4 & $A_{1}$ & $2 \underline{A_{1}}$ & 4 & 3 & $\frac{0}{0} \frac{0}{2} \frac{0}{1} \frac{0}{3} \frac{0}{4} \frac{0}{5} \| \frac{1}{1} \frac{4}{2}$ \\
\hline 47 & 4 & $A_{1}$ & $2 \underline{A_{1}}$ & 3 & 4 & $\frac{0}{0} \frac{0}{2} \frac{0}{1} \frac{0}{3} \frac{0}{4} \frac{0}{5} \| \frac{1}{4} \frac{4}{7}$ \\
\hline 48 & 4 & $A_{1}$ & $\underline{A_{2}}$ & 4 & 2 & $\frac{0}{0} \frac{0}{2} \frac{0}{1} \frac{0}{3} \frac{0}{4} \frac{0}{5} \| \frac{1}{1} \frac{1}{4}$ \\
\hline 49 & 4 & $A_{1}$ & $3 \underline{A_{1}}$ & 2 & 3 & $\frac{0}{0} \frac{0}{2} \frac{0}{1} \frac{0}{3} \frac{0}{4} \frac{0}{5} \| \frac{1}{1} \frac{4}{2} \frac{4}{7}$ \\
\hline 50 & 4 & $A_{1}$ & $\underline{A_{1}}+\underline{A_{2}}$ & 2 & 2 & $\frac{0}{0} \frac{0}{2} \frac{0}{1} \frac{0}{3} \frac{0}{4} \frac{0}{5} \| \frac{1}{1} \frac{1}{4} \frac{4}{7}$ \\
\hline 51 & 4 & $A_{1}$ & $\underline{A_{3}}$ & 2 & 1 & $\frac{0}{0} \frac{0}{2} \frac{0}{1} \frac{0}{3} \frac{0}{4} \frac{0}{5} \| \frac{3}{7} \frac{1}{1} \frac{1}{4}$ \\
\hline 52 & 4 & $A_{1}$ & $\underline{A_{1}}+\underline{A_{3}}$ & 1 & 1 & $\frac{0}{0} \frac{0}{2} \frac{0}{1} \frac{0}{3} \frac{0}{4} \frac{0}{5} \| \frac{3}{7} \frac{1}{1} \frac{1}{4} \frac{4}{7}$ \\
\hline 53 & 4 & $2 A_{1}$ & $A_{0}$ & 4 & 2 & $\frac{0}{0} \frac{0}{2} \frac{0}{1} \frac{0}{4} \frac{0}{3} \frac{0}{5} \|$ \\
\hline 54 & 4 & $2 A_{1}$ & $\underline{A_{1}}$ & 2 & 2 & $\frac{0}{0} \frac{0}{2} \frac{0}{1} \frac{0}{4} \frac{0}{3} \frac{0}{5} \| \frac{4}{2}$ \\
\hline 55 & 4 & $2 A_{1}$ & $2 A_{1}$ & 3 & 2 & $\frac{0}{0} \frac{0}{2} \frac{0}{1} \frac{0}{4} \frac{0}{3} \frac{0}{5} \| \frac{4}{3} \frac{4}{6}$ \\
\hline 56 & 4 & $2 A_{1}$ & $2 \underline{A_{1}}$ & 1 & 2 & $\frac{0}{0} \frac{0}{2} \frac{0}{1} \frac{0}{4} \frac{0}{3} \frac{0}{5} \| \frac{4}{2} \frac{4}{7}$ \\
\hline 57 & 4 & $2 A_{1}$ & $A_{3}$ & 1 & 2 & $\frac{0}{0} \frac{0}{2} \frac{0}{1} \frac{0}{4} \frac{0}{3} \frac{0}{5} \| \frac{1}{0} \frac{1}{3} \frac{4}{2}$ \\
\hline 58 & 4 & $2 A_{1}$ & $4 A_{1}$ & 2 & 2 & $\frac{0}{0} \frac{0}{2} \frac{0}{1} \frac{0}{4} \frac{0}{3} \frac{0}{5} \| \frac{1}{0} \frac{1}{3} \frac{4}{4} \frac{4}{5}$ \\
\hline 59 & 4 & $2 A_{1}^{\prime}$ & $A_{0}$ & 0 & 6 & $\frac{9}{5} \frac{3}{6} \frac{3}{5} \frac{3}{4} \frac{0}{5} \frac{0}{4} \|$ \\
\hline 60 & 4 & $2 A_{1}^{\prime}$ & $\underline{A_{1}}$ & 0 & 4 & $\frac{9}{5} \frac{3}{6} \frac{3}{5} \frac{3}{4} \frac{0}{5} \frac{0}{4} \| \frac{0}{9}$ \\
\hline 61 & 4 & $2 A_{1}^{\prime}$ & $2 A_{1}$ & 0 & 5 & $\frac{9}{5} \frac{3}{6} \frac{3}{5} \frac{3}{4} \frac{0}{5} \frac{0}{4} \| \frac{1}{1} \frac{4}{2}$ \\
\hline 62 & 4 & $2 A_{1}^{\prime}$ & $2 \underline{A_{1}}$ & 0 & 3 & $\frac{9}{5} \frac{3}{6} \frac{3}{5} \frac{3}{4} \frac{0}{5} \frac{0}{4} \| \frac{0}{9} \frac{4}{7}$ \\
\hline 63 & 4 & $2 A_{1}^{\prime}$ & $\underline{A_{2}}$ & 0 & 2 & $\frac{9}{5} \frac{3}{6} \frac{3}{5} \frac{3}{4} \frac{0}{5} \frac{0}{4} \| \frac{0}{9} \frac{1}{0}$ \\
\hline 64 & 4 & $2 A_{1}^{\prime}$ & $2 A_{1}+\underline{A_{1}}$ & 0 & 3 & $\frac{9}{5} \frac{3}{6} \frac{3}{5} \frac{3}{4} \frac{0}{5} \frac{0}{4} \| \frac{0}{9} \frac{1}{1} \frac{4}{2}$ \\
\hline 65 & 4 & $2 A_{1}^{\prime}$ & $A_{3}$ & 0 & 3 & $\frac{9}{5} \frac{3}{6} \frac{3}{5} \frac{3}{4} \frac{0}{5} \frac{0}{4} \| \frac{0}{9} \frac{1}{2} \frac{4}{3}$ \\
\hline 66 & 4 & $2 A_{1}^{\prime}$ & $\underline{A_{3}}$ & 0 & 1 & $\frac{9}{5} \frac{3}{6} \frac{3}{5} \frac{3}{4} \frac{0}{5} \frac{0}{4} \| \frac{1}{0} \frac{0}{9} \frac{4}{5}$ \\
\hline 67 & 4 & $2 A_{1}^{\prime}$ & $4 A_{1}$ & 0 & 4 & $\frac{9}{5} \frac{3}{6} \frac{3}{5} \frac{3}{4} \frac{0}{5} \frac{0}{4} \| \frac{1}{1} \frac{1}{6} \frac{3}{9} \frac{4}{2}$ \\
\hline 68 & 4 & $2 A_{1}^{\prime}$ & $2 A_{1}+2 \underline{A_{1}}$ & 0 & 2 & $\frac{9}{5} \frac{3}{6} \frac{3}{5} \frac{3}{4} \frac{0}{5} \frac{0}{4} \| \frac{0}{9} \frac{4}{7} \frac{1}{1} \frac{4}{2}$ \\
\hline 69 & 4 & $2 A_{1}^{\prime}$ & $\mathrm{D}_{4}$ & 0 & 1 & $\frac{9}{5} \frac{3}{6} \frac{3}{5} \frac{3}{4} \frac{0}{5} \frac{0}{4} \| \frac{0}{9} \frac{1}{0} \frac{1}{1} \frac{4}{2}$ \\
\hline 70 & 4 & $2 A_{1}^{\prime}$ & $2 A_{1}+\ldots A_{3}$ & 0 & 2 & $\frac{9}{5} \frac{3}{6} \frac{3}{5} \frac{3}{4} \frac{0}{5} \frac{0}{4} \| \frac{0}{9} \frac{1}{1} \frac{1}{6} \frac{3}{9} \frac{4}{2}$ \\
\hline 71 & 4 & $3 A_{1}$ & $A_{0}$ & 0 & 2 & $\frac{9}{5} \frac{3}{5} \frac{3}{6} \frac{3}{4} \frac{0}{5} \frac{0}{4} \|$ \\
\hline 72 & 4 & $3 A_{1}$ & $\underline{A_{1}}$ & 0 & 2 & $\frac{9}{5} \frac{3}{5} \frac{3}{6} \frac{3}{4} \frac{0}{5} \frac{0}{4} \| \frac{4}{7}$ \\
\hline 73 & 4 & $3 A_{1}$ & $2 A_{1}$ & 0 & 1 & $\frac{9}{5} \frac{3}{5} \frac{3}{6} \frac{3}{4} \frac{0}{5} \frac{0}{4} \| \mid \frac{1}{1} \frac{4}{2}$ \\
\hline 74 & 4 & $3 A_{1}$ & $A_{2}$ & 0 & 2 & $\frac{9}{5} \frac{3}{5} \frac{3}{6} \frac{3}{4} \frac{0}{5} \frac{0}{4}|| \frac{1}{2} \frac{4}{4}$ \\
\hline 75 & 4 & $3 A_{1}$ & $2 A_{1}+\underline{A_{1}}$ & 0 & 1 & $\frac{9}{5} \frac{3}{5} \frac{3}{6} \frac{3}{4} \frac{0}{5} \frac{0}{4} \| \frac{4}{7} \frac{1}{1} \frac{4}{2}$ \\
\hline 76 & 4 & $3 A_{1}$ & $2 A_{1}+A_{2}$ & 0 & 1 & $\frac{9}{5} \frac{3}{5} \frac{3}{6} \frac{3}{4} \frac{0}{5} \frac{0}{4} \| \frac{1}{1} \frac{1}{6} \frac{4}{0} \frac{4}{2}$ \\
\hline 77 & 4 & $D_{4}$ & $A_{0}$ & 0 & 2 & $\frac{3}{f} \frac{9}{6} \frac{3}{4} \frac{3}{5} \frac{3}{8} \frac{4}{1} \|$ \\
\hline 78 & 4 & $D_{4}$ & $2 A_{1}$ & 0 & 1 & $\frac{3}{f} \frac{9}{6} \frac{3}{4} \frac{3}{5} \frac{3}{8} \frac{4}{1} \| \frac{0}{9} \frac{4}{7}$ \\
\hline
\end{tabular}




\begin{tabular}{|c|c|c|c|c|c|c|}
\hline & $\mathrm{d}$ & $D(A)$ & $D(B)$ & $\# E$ & $\# G$ & $\sigma_{A} \| B$ \\
\hline 79 & 3 & $A_{0}$ & $A_{0}$ & 27 & 27 & $\frac{0}{0} \frac{0}{1} \frac{0}{2} \frac{0}{3} \frac{0}{4} \frac{0}{5} \frac{0}{6} \|$ \\
\hline 80 & 3 & $A_{0}$ & $\underline{A_{1}}$ & 21 & 21 & $\frac{0}{0} \frac{0}{1} \frac{0}{2} \frac{0}{3} \frac{0}{4} \frac{0}{5} \frac{0}{6} \| \frac{0}{9}$ \\
\hline 81 & 3 & $A_{0}$ & $2 \underline{A_{1}}$ & 16 & 16 & $\frac{0}{0} \frac{0}{1} \frac{0}{2} \frac{0}{3} \frac{0}{4} \frac{0}{5} \frac{0}{6} \| \frac{0}{9} \frac{1}{1}$ \\
\hline 82 & 3 & $A_{0}$ & $\underline{A_{2}}$ & 15 & 15 & $\frac{0}{0} \frac{0}{1} \frac{0}{2} \frac{0}{3} \frac{0}{4} \frac{0}{5} \frac{0}{6} \| \frac{0}{9} \frac{1}{0}$ \\
\hline 83 & 3 & $A_{0}$ & $3 \underline{A_{1}}$ & 12 & 12 & $\frac{0}{0} \frac{0}{1} \frac{0}{2} \frac{0}{3} \frac{0}{4} \frac{0}{5} \frac{0}{6} \| \frac{0}{9} \frac{1}{1} \frac{1}{7}$ \\
\hline 84 & 3 & $A_{0}$ & $\underline{A_{1}}+\underline{A_{2}}$ & 11 & 11 & $\frac{0}{0} \frac{0}{1} \frac{0}{2} \frac{0}{3} \frac{0}{4} \frac{0}{5} \frac{0}{6} \| \frac{0}{9} \frac{1}{0} \frac{1}{4}$ \\
\hline 85 & 3 & $A_{0}$ & $\underline{A_{3}}$ & 10 & 10 & $\frac{0}{0} \frac{0}{1} \frac{0}{2} \frac{0}{3} \frac{0}{4} \frac{0}{5} \frac{0}{6} \| \frac{0}{9} \frac{1}{0} \frac{1}{1}$ \\
\hline 86 & 3 & $A_{0}$ & $2 \underline{A_{1}}+\underline{A_{2}}$ & 8 & 8 & $\frac{0}{0} \frac{0}{1} \frac{0}{2} \frac{0}{3} \frac{0}{4} \frac{0}{5} \frac{0}{6} \| \frac{0}{9} \frac{1}{0} \frac{1}{4} \frac{3}{7}$ \\
\hline 87 & 3 & $A_{0}$ & $2 \underline{A_{2}}$ & 7 & 7 & $\frac{0}{0} \frac{0}{1} \frac{0}{2} \frac{0}{3} \frac{0}{4} \frac{0}{5} \frac{0}{6} \| \frac{0}{9} \frac{1}{0} \frac{1}{4} \frac{1}{7}$ \\
\hline 88 & 3 & $A_{0}$ & $4 \underline{A_{1}}$ & 9 & 9 & $\frac{0}{0} \frac{0}{1} \frac{0}{2} \frac{0}{3} \frac{0}{4} \frac{0}{5} \frac{0}{6} \| \frac{0}{9} \frac{1}{1} \frac{1}{7} \frac{9}{7}$ \\
\hline 89 & 3 & $A_{0}$ & $\underline{A_{1}}+\underline{A_{3}}$ & 7 & 7 & $\frac{0}{0} \frac{0}{1} \frac{0}{2} \frac{0}{3} \frac{0}{4} \frac{0}{5} \frac{0}{6} \| \frac{0}{9} \frac{1}{0} \frac{1}{1} \frac{1}{7}$ \\
\hline 90 & 3 & $A_{0}$ & $\underline{A_{4}}$ & 6 & 6 & $\frac{0}{0} \frac{0}{1} \frac{0}{2} \frac{0}{3} \frac{0}{4} \frac{0}{5} \frac{0}{6} \| \frac{0}{9} \frac{1}{0} \frac{1}{1} \frac{1}{4}$ \\
\hline 91 & 3 & $A_{0}$ & $\underline{D_{4}}$ & 6 & 6 & $\frac{0}{0} \frac{0}{1} \frac{0}{2} \frac{0}{3} \frac{0}{4} \frac{0}{5} \frac{0}{6} \| \frac{1}{0} \frac{1}{1} \frac{1}{4} \frac{3}{7}$ \\
\hline 92 & 3 & $A_{0}$ & $2 \underline{A_{1}}+\underline{A_{3}}$ & 5 & 5 & $\frac{0}{0} \frac{0}{1} \frac{0}{2} \frac{0}{3} \frac{0}{4} \frac{0}{5} \frac{0}{6} \| \frac{0}{9} \frac{1}{0} \frac{1}{1} \frac{1}{7} \frac{9}{7}$ \\
\hline 93 & 3 & $A_{0}$ & $\underline{A_{1}}+2 \underline{A_{2}}$ & 5 & 5 & $\frac{0}{0} \frac{0}{1} \frac{0}{2} \frac{0}{3} \frac{0}{4} \frac{0}{5} \frac{0}{6} \| \frac{0}{9} \frac{1}{0} \frac{1}{4} \frac{1}{7} \frac{3}{7}$ \\
\hline 94 & 3 & $A_{0}$ & $\underline{A_{1}}+\underline{A_{4}}$ & 4 & 4 & $\frac{0}{0} \frac{0}{1} \frac{0}{2} \frac{0}{3} \frac{0}{4} \frac{0}{5} \frac{0}{6} \| \frac{0}{9} \frac{1}{0} \frac{1}{1} \frac{1}{7} \frac{3}{7}$ \\
\hline 95 & 3 & $A_{0}$ & $\underline{A_{5}}$ & 3 & 3 & $\frac{0}{0} \frac{0}{1} \frac{0}{2} \frac{0}{3} \frac{0}{4} \frac{0}{5} \frac{0}{6} \| \frac{0}{9} \frac{1}{0} \frac{1}{1} \frac{1}{4} \frac{1}{7}$ \\
\hline 96 & 3 & $A_{0}$ & $\underline{D_{5}}$ & 3 & 3 & $\frac{0}{0} \frac{0}{1} \frac{0}{2} \frac{0}{3} \frac{0}{4} \frac{0}{5} \frac{0}{6} \| \frac{0}{9} \frac{1}{0} \frac{1}{1} \frac{1}{4} \frac{3}{7}$ \\
\hline 97 & 3 & $A_{0}$ & $3 \underline{A_{2}}$ & 3 & 3 & $\frac{0}{0} \frac{0}{1} \frac{0}{2} \frac{0}{3} \frac{0}{4} \frac{0}{5} \frac{0}{6} \| \frac{0}{9} \frac{1}{0} \frac{1}{4} \frac{1}{7} \frac{3}{7} \frac{5}{7}$ \\
\hline 98 & 3 & $A_{0}$ & $\underline{A_{1}}+\underline{A_{5}}$ & 2 & 2 & $\frac{0}{0} \frac{0}{1} \frac{0}{2} \frac{0}{3} \frac{0}{4} \frac{0}{5} \frac{0}{6} \| \frac{0}{9} \frac{1}{0} \frac{1}{1} \frac{1}{4} \frac{1}{7} \frac{9}{7}$ \\
\hline 99 & 3 & $A_{0}$ & $\underline{E_{6}}$ & 1 & 1 & $\frac{0}{0} \frac{0}{1} \frac{0}{2} \frac{0}{3} \frac{0}{4} \frac{0}{5} \frac{0}{6} \| \frac{0}{9} \frac{1}{0} \frac{1}{1} \frac{1}{4} \frac{1}{7} \frac{3}{7}$ \\
\hline 100 & 3 & $A_{1}$ & $A_{0}$ & 15 & 15 & $\frac{0}{0} \frac{0}{2} \frac{0}{1} \frac{0}{3} \frac{0}{4} \frac{0}{5} \frac{0}{6} \|$ \\
\hline 101 & 3 & $A_{1}$ & $\underline{A_{1}}$ & 11 & 11 & $\frac{0}{0} \frac{0}{2} \frac{0}{1} \frac{0}{3} \frac{0}{4} \frac{0}{5} \frac{0}{6} \| \frac{1}{7}$ \\
\hline 102 & 3 & $A_{1}$ & $2 \underline{A_{1}}$ & 8 & 8 & $\frac{0}{0} \frac{0}{2} \frac{0}{1} \frac{0}{3} \frac{0}{4} \frac{0}{5} \frac{0}{6} \| \frac{1}{7} \frac{5}{6}$ \\
\hline 103 & 3 & $A_{1}$ & $\underline{A_{2}}$ & 7 & 7 & $\frac{0}{0} \frac{0}{2} \frac{0}{1} \frac{0}{3} \frac{0}{4} \frac{0}{5} \frac{0}{6} \| \frac{1}{4} \frac{1}{7}$ \\
\hline 104 & 3 & $A_{1}$ & $3 \underline{A_{1}}$ & 6 & 6 & $\frac{0}{0} \frac{0}{2} \frac{0}{1} \frac{0}{3} \frac{0}{4} \frac{0}{5} \frac{0}{6} \| \frac{1}{4} \frac{1}{9} \frac{9}{7}$ \\
\hline 105 & 3 & $A_{1}$ & $\underline{A_{1}}+\underline{A_{2}}$ & 5 & 5 & $\frac{0}{0} \frac{0}{2} \frac{0}{1} \frac{0}{3} \frac{0}{4} \frac{0}{5} \frac{0}{6} \| \frac{1}{4} \frac{1}{7} \frac{5}{7}$ \\
\hline 106 & 3 & $A_{1}$ & $\underline{A_{3}}$ & 4 & 4 & $\frac{0}{0} \frac{0}{2} \frac{0}{1} \frac{0}{3} \frac{0}{4} \frac{0}{5} \frac{0}{6} \| \frac{1}{4} \frac{4}{7} \frac{1}{7}$ \\
\hline 107 & 3 & $A_{1}$ & $2 \underline{A_{2}}$ & 3 & 3 & $\frac{0}{0} \frac{0}{2} \frac{0}{1} \frac{0}{3} \frac{0}{4} \frac{0}{5} \frac{0}{6} \| \frac{1}{1} \frac{4}{2} \frac{1}{7} \frac{5}{6}$ \\
\hline 108 & 3 & $A_{1}$ & $\underline{A_{1}}+\underline{A_{3}}$ & 3 & 3 & $\frac{0}{0} \frac{0}{2} \frac{0}{1} \frac{0}{3} \frac{0}{4} \frac{0}{5} \frac{0}{6} \| \frac{1}{1} \frac{1}{4} \frac{1}{7} \frac{9}{7}$ \\
\hline 109 & 3 & $A_{1}$ & $\underline{A_{4}}$ & 2 & 2 & $\frac{0}{0} \frac{0}{2} \frac{0}{1} \frac{0}{3} \frac{0}{4} \frac{0}{5} \frac{0}{6} \| \frac{1}{1} \frac{1}{4} \frac{4}{7} \frac{1}{7}$ \\
\hline 110 & 3 & $A_{1}$ & $\underline{A_{5}}$ & 1 & 1 & $\frac{0}{0} \frac{0}{2} \frac{0}{1} \frac{0}{3} \frac{0}{4} \frac{0}{5} \frac{0}{6} \| \frac{3}{7} \frac{1}{1} \frac{1}{4} \frac{4}{7} \frac{1}{7}$ \\
\hline 111 & 3 & $2 A_{1}$ & $A_{0}$ & 7 & 7 & $\frac{0}{0} \frac{0}{2} \frac{0}{1} \frac{0}{4} \frac{0}{3} \frac{0}{5} \frac{0}{6} \|$ \\
\hline 112 & 3 & $2 A_{1}$ & $\underline{A_{1}}$ & 5 & 5 & $\frac{0}{0} \frac{0}{2} \frac{0}{1} \frac{0}{4} \frac{0}{3} \frac{0}{5} \frac{0}{6} \| \frac{1}{7}$ \\
\hline 113 & 3 & $2 A_{1}$ & $2 A_{1}$ & 6 & 6 & $\frac{0}{0} \frac{0}{2} \frac{0}{1} \frac{0}{4} \frac{0}{3} \frac{0}{5} \frac{0}{6} \| \frac{5}{0} \frac{5}{3}$ \\
\hline 114 & 3 & $2 A_{1}$ & $2 \underline{A_{1}}$ & 4 & 4 & $\frac{0}{0} \frac{0}{2} \frac{0}{1} \frac{0}{4} \frac{0}{3} \frac{0}{5} \frac{0}{6} \| \frac{1}{7} \frac{9}{7}$ \\
\hline 115 & 3 & $2 A_{1}$ & $\underline{A_{2}}$ & 3 & 3 & $\frac{0}{0} \frac{0}{2} \frac{0}{1} \frac{0}{4} \frac{0}{3} \frac{0}{5} \frac{0}{6} \| \frac{4}{2} \frac{1}{7}$ \\
\hline 116 & 3 & $2 A_{1}$ & $2 A_{1}+\underline{A_{1}}$ & 4 & 4 & $\frac{0}{0} \frac{0}{2} \frac{0}{1} \frac{0}{4} \frac{0}{3} \frac{0}{5} \frac{0}{6} \| \frac{4}{2} \frac{5}{0} \frac{5}{3}$ \\
\hline 117 & 3 & $2 A_{1}$ & $A_{3}$ & 4 & 4 & \\
\hline 118 & 3 & $2 A_{1}$ & $\underline{A_{3}}$ & 2 & 2 & $\frac{0}{0} \frac{0}{2} \frac{0}{1} \frac{0}{4} \frac{0}{3} \frac{0}{5} \frac{0}{6} \| \frac{4}{2} \frac{4}{7} \frac{1}{7}$ \\
\hline 119 & 3 & $2 A_{1}$ & $4 A_{1}$ & 5 & 5 & $\frac{0}{0} \frac{0}{2} \frac{0}{1} \frac{0}{4} \frac{0}{3} \frac{0}{5} \frac{0}{6} \| \frac{4}{3} \frac{4}{6} \frac{5}{1} \frac{5}{2}$ \\
\hline 120 & 3 & $2 A_{1}$ & $2 \underline{A_{1}}+2 A_{1}$ & 3 & 3 & $\frac{0}{0} \frac{0}{2} \frac{0}{1} \frac{0}{4} \frac{0}{3} \frac{0}{5} \frac{0}{6} \| \frac{4}{3} \frac{4}{6} \frac{4}{9} \frac{5}{4}$ \\
\hline 121 & 3 & $2 A_{1}$ & $D_{4}$ & 2 & 2 & $\frac{0}{0} \frac{0}{2} \frac{0}{1} \frac{0}{4} \frac{0}{3} \frac{0}{5} \frac{0}{6} \| \frac{1}{0} \frac{1}{3} \frac{4}{2} \frac{1}{7}$ \\
\hline 122 & 3 & $2 A_{1}$ & $2 A_{1}+A_{3}$ & 3 & 3 & $\frac{0}{0} \frac{0}{2} \frac{0}{1} \frac{0}{4} \frac{0}{3} \frac{0}{5} \frac{0}{6} \| \frac{1}{0} \frac{1}{3} \frac{4}{2} \frac{5}{1} \frac{5}{2}$ \\
\hline 123 & 3 & $3 A_{1}$ & $A_{0}$ & 3 & 3 & $\frac{0}{0} \frac{0}{2} \frac{0}{1} \frac{0}{4} \frac{0}{3} \frac{0}{6} \frac{0}{5} \|$ \\
\hline 124 & 3 & $3 A_{1}$ & $\underline{A_{1}}$ & 3 & 3 & $\frac{0}{0} \frac{0}{2} \frac{0}{1} \frac{0}{4} \frac{0}{3} \frac{0}{6} \frac{0}{5} \| \frac{9}{7}$ \\
\hline 125 & 3 & $3 A_{1}$ & $2 A_{1}$ & 2 & 2 & $\frac{0}{0} \frac{0}{2} \frac{0}{1} \frac{0}{4} \frac{0}{3} \frac{0}{6} \frac{0}{5} \| \frac{1}{0} \frac{1}{3}$ \\
\hline 126 & 3 & $3 A_{1}$ & $A_{2}$ & 3 & 3 & $\frac{0}{0} \frac{0}{2} \frac{0}{1} \frac{0}{4} \frac{0}{3} \frac{0}{6} \frac{0}{5} \| \frac{4}{3} \frac{5}{3}$ \\
\hline 127 & 3 & $3 A_{1}$ & $2 A_{1}+\underline{A_{1}}$ & 2 & 2 & $\frac{0}{0} \frac{0}{2} \frac{0}{1} \frac{0}{4} \frac{0}{3} \frac{0}{6} \frac{0}{5} \| \frac{9}{7} \frac{1}{0} \frac{1}{3}$ \\
\hline 128 & 3 & $3 A_{1}$ & $2 A_{1}+A_{2}$ & 2 & 2 & $\frac{0}{0} \frac{0}{2} \frac{0}{1} \frac{0}{4} \frac{0}{3} \frac{0}{6} \frac{0}{5} \| \frac{1}{0} \frac{1}{3} \frac{4}{4} \frac{5}{2}$ \\
\hline 129 & 3 & $3 A_{1}$ & $2 A_{2}$ & 1 & 1 & $\frac{0}{0} \frac{0}{2} \frac{0}{1} \frac{0}{4} \frac{0}{3} \frac{0}{6} \frac{0}{5} \| \frac{1}{0} \frac{1}{3} \frac{1}{4} \frac{1}{9}$ \\
\hline 130 & 3 & $3 A_{1}$ & $\underline{A_{1}}+2 A_{2}$ & 1 & 1 & $\frac{0}{0} \frac{0}{2} \frac{0}{1} \frac{0}{4} \frac{0}{3} \frac{0}{6} \frac{0}{5} \| \frac{9}{7} \frac{1}{0} \frac{1}{3} \frac{1}{4} \frac{1}{9}$ \\
\hline 131 & 3 & $3 A_{1}$ & $A_{2}+2 A_{2}$ & 1 & 1 & $\frac{0}{0} \frac{0}{2} \frac{0}{1} \frac{0}{4} \frac{0}{3} \frac{0}{6} \frac{0}{5} \| \frac{1}{0} \frac{1}{3} \frac{1}{4} \frac{1}{9} \frac{4}{5} \frac{5}{1}$ \\
\hline 132 & 3 & $D_{4}$ & $A_{0}$ & 3 & 3 & $\frac{3}{f} \frac{9}{6} \frac{3}{4} \frac{3}{5} \frac{3}{8} \frac{4}{1} \frac{0}{6} \|$ \\
\hline 133 & 3 & $D_{4}$ & $2 A_{1}$ & 2 & 2 & $\frac{3}{f} \frac{9}{6} \frac{3}{4} \frac{3}{5} \frac{3}{8} \frac{4}{1} \frac{0}{6} \| \frac{1}{7} \frac{5}{5}$ \\
\hline 134 & 3 & $D_{4}$ & $2 A_{2}$ & 1 & 1 & $\frac{3}{f} \frac{9}{6} \frac{3}{4} \frac{3}{5} \frac{3}{8} \frac{4}{1} \frac{0}{6} \| \frac{0}{9} \frac{4}{7} \frac{1}{7} \frac{5}{5}$ \\
\hline
\end{tabular}

\begin{tabular}{|c|c|c|c|c|c|c|}
\hline & $\mathrm{d}$ & $D(A)$ & $D(B)$ & $\# E$ & $\# G$ & $\sigma_{A} \| B$ \\
\hline 135 & 2 & $A_{0}$ & $A_{0}$ & 56 & 126 & $\frac{0}{0} \frac{0}{1} \frac{0}{2} \frac{0}{3} \frac{0}{4} \frac{0}{5} \frac{0}{6} \frac{0}{7} \|$ \\
\hline 136 & 2 & $A_{0}$ & $\underline{A_{1}}$ & 44 & 93 & $\frac{0}{0} \frac{0}{1} \frac{0}{2} \frac{0}{3} \frac{0}{4} \frac{0}{5} \frac{0}{6} \frac{0}{7} \| \frac{0}{9}$ \\
\hline 137 & 2 & $A_{0}$ & $2 \underline{A_{1}}$ & 34 & 68 & $\frac{0}{0} \frac{0}{1} \frac{0}{2} \frac{0}{3} \frac{0}{4} \frac{0}{5} \frac{0}{6} \frac{0}{7} \| \frac{0}{9} \frac{1}{1}$ \\
\hline 138 & 2 & $A_{0}$ & $\underline{A_{2}}$ & 32 & 61 & $\frac{0}{0} \frac{0}{1} \frac{0}{2} \frac{0}{3} \frac{0}{4} \frac{0}{5} \frac{0}{6} \frac{0}{7} \| \frac{0}{9} \frac{1}{0}$ \\
\hline 139 & 2 & $A_{0}$ & $3 \underline{A_{1}}$ & 26 & 49 & $\frac{0}{0} \frac{0}{1} \frac{0}{2} \frac{0}{3} \frac{0}{4} \frac{0}{5} \frac{0}{6} \frac{0}{7} \| \frac{0}{9} \frac{1}{1} \frac{1}{7}$ \\
\hline 140 & 2 & $A_{0}$ & $3 \underline{A_{1}}$ & 25 & 51 & $\frac{0}{0} \frac{0}{1} \frac{0}{2} \frac{0}{3} \frac{0}{4} \frac{0}{5} \frac{0}{6} \frac{0}{7} \| \frac{1}{4} \frac{2}{2} \frac{3}{7}$ \\
\hline 141 & 2 & $A_{0}$ & $\underline{A_{1}}+\underline{A_{2}}$ & 24 & 44 & $\frac{0}{0} \frac{0}{1} \frac{0}{2} \frac{0}{3} \frac{0}{4} \frac{0}{5} \frac{0}{6} \frac{0}{7} \| \frac{0}{9} \frac{1}{0} \frac{1}{4}$ \\
\hline 142 & 2 & $A_{0}$ & $\underline{A_{3}}$ & 22 & 37 & $\frac{0}{0} \frac{0}{1} \frac{0}{2} \frac{0}{3} \frac{0}{4} \frac{0}{5} \frac{0}{6} \frac{0}{7} \| \frac{0}{9} \frac{1}{0} \frac{1}{1}$ \\
\hline 143 & 2 & $A_{0}$ & $2 \underline{A_{1}}+\underline{A_{2}}$ & 18 & 31 & $\frac{0}{0} \frac{0}{1} \frac{0}{2} \frac{0}{3} \frac{0}{4} \frac{0}{5} \frac{0}{6} \frac{0}{7} \| \frac{0}{9} \frac{1}{0} \frac{2}{4} \frac{2}{2}$ \\
\hline 144 & 2 & $A_{0}$ & $2 \underline{A_{2}}$ & 16 & 28 & $\frac{0}{0} \frac{0}{1} \frac{0}{2} \frac{0}{3} \frac{0}{4} \frac{0}{5} \frac{0}{6} \frac{0}{7} \| \frac{0}{9} \frac{1}{0} \frac{1}{4} \frac{1}{7}$ \\
\hline 145 & 2 & $A_{0}$ & $4 \underline{A_{1}}$ & 20 & 35 & $\frac{0}{0} \frac{0}{1} \frac{0}{2} \frac{0}{3} \frac{0}{4} \frac{0}{5} \frac{0}{6} \frac{0}{7} \| \frac{0}{9} \frac{1}{1} \frac{1}{7} \frac{9}{7}$ \\
\hline 146 & 2 & $A_{0}$ & $4 \underline{A_{1}}$ & 19 & 36 & $\frac{0}{0} \frac{0}{1} \frac{0}{2} \frac{0}{3} \frac{0}{4} \frac{0}{5} \frac{0}{6} \frac{0}{7} \| \frac{0}{9} \frac{1}{4} \frac{2}{2} \frac{3}{7}$ \\
\hline 147 & 2 & $A_{0}$ & $\underline{A_{1}}+\underline{A_{3}}$ & 16 & 26 & $\frac{0}{0} \frac{0}{1} \frac{0}{2} \frac{0}{3} \frac{0}{4} \frac{0}{5} \frac{0}{6} \frac{0}{7} \| \frac{0}{9} \frac{1}{0} \frac{1}{1} \frac{1}{7}$ \\
\hline 148 & 2 & $A_{0}$ & $\underline{A_{1}}+\underline{A_{3}}$ & 15 & 28 & $\frac{0}{0} \frac{0}{1} \frac{0}{2} \frac{0}{3} \frac{0}{4} \frac{0}{5} \frac{0}{6} \frac{0}{7} \| \frac{1}{1} \frac{1}{4} \frac{2}{2} \frac{3}{7}$ \\
\hline 149 & 2 & $A_{0}$ & $\underline{A_{4}}$ & 14 & 21 & $\frac{0}{0} \frac{0}{1} \frac{0}{2} \frac{0}{3} \frac{0}{4} \frac{0}{5} \frac{0}{6} \frac{0}{7} \| \frac{0}{9} \frac{1}{0} \frac{1}{1} \frac{1}{4}$ \\
\hline 150 & 2 & $A_{0}$ & $\underline{D_{4}}$ & 14 & 19 & $\frac{0}{0} \frac{0}{1} \frac{0}{2} \frac{0}{3} \frac{0}{4} \frac{0}{5} \frac{0}{6} \frac{0}{7} \| \frac{1}{0} \frac{1}{1} \frac{1}{4} \frac{3}{7}$ \\
\hline 151 & 2 & $A_{0}$ & $2 \underline{A_{1}}+\underline{A_{3}}$ & 12 & 18 & $\frac{0}{0} \frac{0}{1} \frac{0}{2} \frac{0}{3} \frac{0}{4} \frac{0}{5} \frac{0}{6} \frac{0}{7} \| \frac{0}{9} \frac{1}{0} \frac{1}{1} \frac{1}{7} \frac{9}{7}$ \\
\hline 152 & 2 & $A_{0}$ & $2 \underline{A_{1}}+\underline{A_{3}}$ & 11 & 19 & $\frac{0}{0} \frac{0}{1} \frac{0}{2} \frac{0}{3} \frac{0}{4} \frac{0}{5} \frac{0}{6} \frac{0}{7} \| \frac{0}{9} \frac{1}{1} \frac{1}{4} \frac{2}{2} \frac{3}{7}$ \\
\hline 153 & 2 & $A_{0}$ & $3 \underline{A_{1}}+\underline{A_{2}}$ & 13 & 22 & $\frac{0}{0} \frac{0}{1} \frac{0}{2} \frac{0}{3} \frac{0}{4} \frac{0}{5} \frac{0}{6} \frac{0}{7} \| \frac{0}{9} \frac{1}{0} \frac{1}{4} \frac{2}{2} \frac{3}{7}$ \\
\hline 154 & 2 & $A_{0}$ & $5 \underline{A_{1}}$ & 14 & 26 & $\frac{0}{0} \frac{0}{1} \frac{0}{2} \frac{0}{3} \frac{0}{4} \frac{0}{5} \frac{0}{6} \frac{0}{7} \| \frac{1}{0} \frac{1}{4} \frac{2}{2} \frac{3}{7} \frac{4}{5}$ \\
\hline 155 & 2 & $A_{0}$ & $\underline{A_{1}}+2 \underline{A_{2}}$ & 12 & 19 & $\frac{0}{0} \frac{0}{1} \frac{0}{2} \frac{0}{3} \frac{0}{4} \frac{0}{5} \frac{0}{6} \frac{0}{7} \| \frac{0}{9} \frac{1}{0} \frac{1}{4} \frac{1}{7} \frac{3}{7}$ \\
\hline 156 & 2 & $A_{0}$ & $\underline{A_{1}}+\underline{A_{4}}$ & 10 & 14 & $\frac{0}{0} \frac{0}{1} \frac{0}{2} \frac{0}{3} \frac{0}{4} \frac{0}{5} \frac{0}{6} \frac{0}{7} \| \frac{0}{9} \frac{1}{0} \frac{1}{1} \frac{1}{4} \frac{2}{2}$ \\
\hline 157 & 2 & $A_{0}$ & $\underline{A_{1}}+\underline{D_{4}}$ & 9 & 14 & $\frac{0}{0} \frac{0}{1} \frac{0}{2} \frac{0}{3} \frac{0}{4} \frac{0}{5} \frac{0}{6} \frac{0}{7} \| \frac{1}{0} \frac{1}{1} \frac{1}{4} \frac{2}{2} \frac{3}{7}$ \\
\hline 158 & 2 & $A_{0}$ & $\underline{A_{2}}+\underline{A_{3}}$ & 10 & 16 & $\frac{0}{0} \frac{0}{1} \frac{0}{2} \frac{0}{3} \frac{0}{4} \frac{0}{5} \frac{0}{6} \frac{0}{7} \| \frac{0}{9} \frac{1}{0} \frac{1}{1} \frac{1}{7} \frac{2}{2}$ \\
\hline 159 & 2 & $A_{0}$ & $\underline{A_{5}}$ & 8 & 11 & $\frac{0}{0} \frac{0}{1} \frac{0}{2} \frac{0}{3} \frac{0}{4} \frac{0}{5} \frac{0}{6} \frac{0}{7} \| \frac{0}{9} \frac{1}{0} \frac{1}{1} \frac{1}{4} \frac{1}{7}$ \\
\hline 160 & 2 & $A_{0}$ & $\underline{A_{5}}$ & 7 & 13 & $\frac{0}{0} \frac{0}{1} \frac{0}{2} \frac{0}{3} \frac{0}{4} \frac{0}{5} \frac{0}{6} \frac{0}{7} \| \frac{1}{1} \frac{1}{4} \frac{1}{7} \frac{2}{2} \frac{3}{7}$ \\
\hline 161 & 2 & $A_{0}$ & $\underline{D_{5}}$ & 8 & 9 & $\frac{0}{0} \frac{0}{1} \frac{0}{2} \frac{0}{3} \frac{0}{4} \frac{0}{5} \frac{0}{6} \frac{0}{7} \| \frac{0}{9} \frac{1}{0} \frac{1}{1} \frac{1}{4} \frac{3}{7}$ \\
\hline 162 & 2 & $A_{0}$ & $2 \underline{A_{1}}+\underline{D_{4}}$ & 6 & 10 & $\frac{0}{0} \frac{0}{1} \frac{0}{2} \frac{0}{3} \frac{0}{4} \frac{0}{5} \frac{0}{6} \frac{0}{7} \| \frac{1}{0} \frac{1}{4} \frac{1}{7} \frac{2}{2} \frac{3}{7} \frac{4}{5}$ \\
\hline 163 & 2 & $A_{0}$ & $2 \underline{A_{3}}$ & 6 & 9 & $\frac{0}{0} \frac{0}{1} \frac{0}{2} \frac{0}{3} \frac{0}{4} \frac{0}{5} \frac{0}{6} \frac{0}{7} \| \frac{0}{9} \frac{1}{0} \frac{1}{1} \frac{1}{7} \frac{2}{2} \frac{9}{7}$ \\
\hline 164 & 2 & $A_{0}$ & $3 \underline{A_{1}}+\underline{A_{3}}$ & 8 & 13 & $\frac{0}{0} \frac{0}{1} \frac{0}{2} \frac{0}{3} \frac{0}{4} \frac{0}{5} \frac{0}{6} \frac{0}{7} \| \frac{0}{9} \frac{1}{0} \frac{1}{4} \frac{2}{2} \frac{3}{7} \frac{4}{5}$ \\
\hline 165 & 2 & $A_{0}$ & $3 \underline{A_{2}}$ & 8 & 11 & $\frac{0}{0} \frac{0}{1} \frac{0}{2} \frac{0}{3} \frac{0}{4} \frac{0}{5} \frac{0}{6} \frac{0}{7} \| \frac{0}{9} \frac{1}{0} \frac{1}{4} \frac{1}{7} \frac{3}{7} \frac{5}{7}$ \\
\hline 166 & 2 & $A_{0}$ & $6 \underline{A_{1}}$ & 10 & 19 & $\frac{0}{0} \frac{0}{1} \frac{0}{2} \frac{0}{3} \frac{0}{4} \frac{0}{5} \frac{0}{6} \frac{0}{7} \| \frac{0}{9} \frac{1}{1} \frac{1}{7} \frac{5}{9} \frac{6}{4} \frac{7}{3}$ \\
\hline 167 & 2 & $A_{0}$ & $\underline{A_{1}}+\underline{A_{2}}+\underline{A_{3}}$ & 7 & 11 & $\frac{0}{0} \frac{0}{1} \frac{0}{2} \frac{0}{3} \frac{0}{4} \frac{0}{5} \frac{0}{6} \frac{0}{7} \| \frac{0}{9} \frac{1}{0} \frac{1}{4} \frac{1}{7} \frac{2}{2} \frac{3}{7}$ \\
\hline 168 & 2 & $A_{0}$ & $\underline{A_{1}}+\underline{A_{5}}$ & 6 & 7 & $\frac{0}{0} \frac{0}{1} \frac{0}{2} \frac{0}{3} \frac{0}{4} \frac{0}{5} \frac{0}{6} \frac{0}{7} \| \frac{0}{9} \frac{1}{0} \frac{1}{1} \frac{1}{4} \frac{1}{7} \frac{9}{7}$ \\
\hline 169 & 2 & $A_{0}$ & $\underline{A_{1}}+\underline{A_{5}}$ & 5 & 8 & $\frac{0}{0} \frac{0}{1} \frac{0}{2} \frac{0}{3} \frac{0}{4} \frac{0}{5} \frac{0}{6} \frac{0}{7} \| \frac{0}{9} \frac{1}{1} \frac{1}{4} \frac{1}{7} \frac{2}{2} \frac{3}{7}$ \\
\hline 170 & 2 & $A_{0}$ & $\underline{A_{1}}+\underline{D_{5}}$ & 5 & 6 & $\frac{0}{0} \frac{0}{1} \frac{0}{2} \frac{0}{3} \frac{0}{4} \frac{0}{5} \frac{0}{6} \frac{0}{7} \| \frac{0}{9} \frac{1}{0} \frac{1}{1} \frac{1}{4} \frac{2}{2} \frac{3}{7}$ \\
\hline 171 & 2 & $A_{0}$ & $\underline{A_{2}}+\underline{A_{4}}$ & 6 & 8 & $\frac{0}{0} \frac{0}{1} \frac{0}{2} \frac{0}{3} \frac{0}{4} \frac{0}{5} \frac{0}{6} \frac{0}{7} \| \frac{0}{9} \frac{1}{0} \frac{1}{1} \frac{1}{7} \frac{2}{2} \frac{3}{7}$ \\
\hline 172 & 2 & $A_{0}$ & $\underline{A_{6}}$ & 4 & 5 & $\frac{0}{0} \frac{0}{1} \frac{0}{2} \frac{0}{3} \frac{0}{4} \frac{0}{5} \frac{0}{6} \frac{0}{7} \| \frac{0}{9} \frac{1}{0} \frac{1}{1} \frac{1}{4} \frac{1}{7} \frac{2}{2}$ \\
\hline 173 & 2 & $A_{0}$ & $\underline{D_{6}}$ & 3 & 5 & $\frac{0}{0} \frac{0}{1} \frac{0}{2} \frac{0}{3} \frac{0}{4} \frac{0}{5} \frac{0}{6} \frac{0}{7} \| \frac{1}{0} \frac{1}{1} \frac{1}{4} \frac{1}{7} \frac{2}{2} \frac{3}{7}$ \\
\hline 174 & 2 & $A_{0}$ & $\underline{E_{6}}$ & 4 & 3 & $\frac{0}{0} \frac{0}{1} \frac{0}{2} \frac{0}{3} \frac{0}{4} \frac{0}{5} \frac{0}{6} \frac{0}{7} \| \frac{0}{9} \frac{1}{0} \frac{1}{1} \frac{1}{4} \frac{1}{7} \frac{3}{7}$ \\
\hline 175 & 2 & $A_{0}$ & $3 \underline{A_{1}}+\underline{D_{4}}$ & 4 & 7 & $\frac{0}{0} \frac{0}{1} \frac{0}{2} \frac{0}{3} \frac{0}{4} \frac{0}{5} \frac{0}{6} \frac{0}{7} \| \frac{0}{9} \frac{1}{1} \frac{1}{4} \frac{1}{7} \frac{5}{9} \frac{6}{4} \frac{9}{7}$ \\
\hline$\times$ & 2 & $A_{0}$ & $7 \underline{A_{1}}$ & 7 & 14 & $\frac{0}{0} \frac{0}{1} \frac{0}{2} \frac{0}{3} \frac{0}{4} \frac{0}{5} \frac{0}{6} \frac{0}{7} \| \frac{0}{9} \frac{1}{1} \frac{1}{7} \frac{5}{9} \frac{6}{4} \frac{7}{3} \frac{9}{7}$ \\
\hline 177 & 2 & $A_{0}$ & $\underline{A_{1}}+2 \underline{A_{3}}$ & 4 & 6 & $\frac{0}{0} \frac{0}{1} \frac{0}{2} \frac{0}{3} \frac{0}{4} \frac{0}{5} \frac{0}{6} \frac{0}{7} \| \frac{0}{9} \frac{1}{0} \frac{1}{1} \frac{1}{7} \frac{2}{2} \frac{7}{3} \frac{9}{7}$ \\
\hline 178 & 2 & $A_{0}$ & $\underline{A_{1}}+\underline{D_{6}}$ & 2 & 3 & $\frac{0}{0} \frac{0}{1} \frac{0}{2} \frac{0}{3} \frac{0}{4} \frac{0}{5} \frac{0}{6} \frac{0}{7} \| \frac{0}{9} \frac{1}{0} \frac{1}{4} \frac{1}{7} \frac{2}{2} \frac{3}{7} \frac{4}{5}$ \\
\hline 179 & 2 & $A_{0}$ & $\underline{A_{2}}+\underline{A_{5}}$ & 3 & 4 & $\frac{0}{0} \frac{0}{1} \frac{0}{2} \frac{0}{3} \frac{0}{4} \frac{0}{5} \frac{0}{6} \frac{0}{7} \| \frac{0}{9} \frac{1}{0} \frac{1}{4} \frac{1}{7} \frac{2}{2} \frac{3}{7} \frac{5}{7}$ \\
\hline 180 & 2 & $A_{0}$ & $\underline{A_{7}}$ & 2 & 2 & $\frac{0}{0} \frac{0}{1} \frac{0}{2} \frac{0}{3} \frac{0}{4} \frac{0}{5} \frac{0}{6} \frac{0}{7} \| \frac{0}{9} \frac{1}{0} \frac{1}{1} \frac{1}{4} \frac{1}{7} \frac{2}{2} \frac{9}{7}$ \\
\hline 181 & 2 & $A_{0}$ & $\underline{E_{7}}$ & 1 & 1 & $\frac{0}{0} \frac{0}{1} \frac{0}{2} \frac{0}{3} \frac{0}{4} \frac{0}{5} \frac{0}{6} \frac{0}{7} \| \frac{0}{9} \frac{1}{0} \frac{1}{1} \frac{1}{4} \frac{1}{7} \frac{2}{2} \frac{3}{7}$ \\
\hline
\end{tabular}




\begin{tabular}{|c|c|c|c|c|c|c|}
\hline & d & $D(A)$ & $D(B)$ & $\# E$ & $\# G$ & $\sigma_{A} \| B$ \\
\hline 182 & 2 & $A_{1}$ & $A_{0}$ & 32 & 60 & $\left.\frac{0}{0} \frac{0}{2} \frac{0}{1} \frac{0}{3} \frac{0}{4} \frac{0}{5} \frac{0}{6}\right) \mid$ \\
\hline 183 & 2 & $A_{1}$ & $\underline{A_{1}}$ & 24 & 43 & $\frac{00}{0} \frac{0}{2} \frac{00000}{1} \frac{0}{4} \frac{0}{6} \| \frac{2}{7}$ \\
\hline 184 & 2 & $A_{1}$ & $2 \underline{A_{1}}$ & 18 & 30 & $\frac{0}{0} \frac{0}{2} \frac{0}{1} \frac{0}{3} \frac{0}{4} \frac{0}{6} \frac{0}{7} \| \frac{2}{2} \frac{7}{1}$ \\
\hline 185 & 2 & $A_{1}$ & $2 \underline{A_{1}}$ & 16 & 34 & $\frac{0}{0} \frac{0}{2} \frac{0}{1} \frac{0}{3} \frac{0}{4} \frac{0}{5} \frac{0}{6}\left\|_{7}\right\| \frac{1}{7} \frac{6}{4}$ \\
\hline 186 & 2 & $A_{1}$ & $\underline{A_{2}}$ & 16 & 27 & $\frac{0}{0} \frac{0}{2} \frac{0}{1} \frac{0}{3} \frac{0}{4} \frac{0}{5} \frac{0}{6} \frac{0}{7} \| \frac{1}{7} \frac{2}{2}$ \\
\hline 187 & 2 & $A_{1}$ & $3 \underline{A_{1}}$ & 14 & 21 & $\frac{0}{0} \frac{0}{2} \frac{0}{1} \frac{0}{3} \frac{0}{4} \frac{0}{5} \frac{0}{6}\|\| \frac{1}{7} \frac{2}{4} \frac{0}{a}$ \\
\hline 188 & 2 & $A_{1}$ & $3 \underline{A_{1}}$ & 13 & 21 & $\frac{0}{0} \frac{0}{2} \frac{0}{1} \frac{0}{3} \frac{0}{4} \frac{0}{5} \frac{0}{6} \frac{0}{7} \| \frac{2}{2} \frac{7}{1} \frac{0}{a}$ \\
\hline 189 & 2 & $A_{1}$ & $3 \underline{A_{1}}$ & 12 & 23 & $\frac{0}{0} \frac{0}{2} \frac{0}{1} \frac{0}{3} \frac{0}{4} \frac{0}{5} \frac{0}{6}\|\| \frac{1}{7} \frac{26}{4}$ \\
\hline 190 & 2 & $A_{1}$ & $\underline{A_{1}}+\underline{A_{2}}$ & 12 & 18 & $\frac{0}{0} \frac{0}{2} \frac{0}{1} \frac{0}{3} \frac{0}{4} \frac{0}{5} \frac{0}{6}\|\| \frac{1}{7} \frac{2}{2} \frac{7}{3}$ \\
\hline 191 & 2 & $A_{1}$ & $\underline{A_{3}}$ & 10 & 15 & $\frac{0}{0} \frac{0}{2} \frac{0}{1} \frac{0}{3} \frac{0}{4} \frac{0}{5} \frac{0}{7}\|\|_{7} \frac{15}{6} \frac{2}{2}$ \\
\hline 192 & 2 & $A_{1}$ & $\underline{A_{3}}$ & 8 & 19 & $\frac{0}{0} \frac{0}{2} \frac{0}{1} \frac{0}{3} \frac{0}{4} \frac{0}{5} \frac{0}{6}\|\|_{4} \frac{1}{7} \frac{1}{7} \frac{1}{4}$ \\
\hline 193 & 2 & $A_{1}$ & $2 \underline{A_{1}}+\underline{A_{2}}$ & 8 & 13 & $\frac{0}{0} \frac{0}{2} \frac{0}{1} \frac{0}{3} \frac{0}{4} \frac{0}{5} \frac{0}{6}\left\|_{7}\right\| \frac{1}{7} \frac{5}{6} \frac{6}{4}$ \\
\hline 194 & 2 & $A_{1}$ & $2 \underline{A_{2}}$ & 8 & 10 & $\frac{000000000}{0} \frac{0}{1} \frac{0}{4} \|^{\frac{1}{7}} \mid \frac{15}{7} \frac{29}{5}$ \\
\hline 195 & 2 & $A_{1}$ & $4 \underline{A_{1}}$ & 9 & 16 & $\left.\frac{0}{0} \frac{0}{2} \frac{0}{1} \frac{0}{3} \frac{0}{4} \frac{0}{5} \frac{0}{6}\right)^{7} \| \frac{1}{7} \frac{26}{4} \frac{0}{4}$ \\
\hline 196 & 2 & $A_{1}$ & $4 \underline{A_{1}}$ & 8 & 17 & $\frac{0}{0} \frac{0}{2} \frac{0}{1} \frac{0}{3} \frac{0}{4} \frac{0}{5} \frac{0}{7}\|\|_{7} \frac{15}{6} \frac{26}{4}$ \\
\hline 197 & 2 & $A_{1}$ & $\underline{A_{1}}+\underline{A_{3}}$ & 8 & 10 & 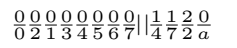 \\
\hline 198 & 2 & $A_{1}$ & $\underline{A_{1}}+\underline{A_{3}}$ & 7 & 10 & $\frac{0}{0} \frac{0}{2} \frac{0}{1} \frac{0}{3} \frac{0}{4} \frac{0}{5} \frac{0}{6}\|\| \frac{1}{7} \frac{5}{6} \frac{0}{2} \frac{0}{a}$ \\
\hline 199 & 2 & $A_{1}$ & $\underline{A_{1}}+\underline{A_{3}}$ & 6 & 12 & $\frac{0}{0} \frac{0}{2} \frac{0}{1} \frac{0}{3} \frac{0}{4} \frac{0}{5} \frac{0}{6}\|\| \frac{1}{7} \frac{12}{7} \frac{6}{4}$ \\
\hline 200 & 2 & $A_{1}$ & $\underline{A_{4}}$ & 6 & 7 & $\frac{0}{7} \| \frac{1}{4} \frac{1}{7} \frac{5}{7} \frac{2}{2}$ \\
\hline 201 & 2 & $A_{1}$ & $\underline{D_{4}}$ & 4 & 9 & $\frac{00}{0} \frac{0}{2} \frac{0}{1} \frac{0}{4} \frac{0}{5} \frac{0}{6} \frac{0}{7} \| \frac{1}{4} \frac{4}{7} \frac{1}{7} \frac{2}{2}$ \\
\hline 202 & 2 & $A_{1}$ & $2 \underline{A_{1}}+\underline{A_{3}}$ & 5 & 7 & $\frac{0}{0} \frac{0}{2} \frac{0}{1} \frac{0}{3} \frac{0}{4} \frac{0}{5} \frac{0}{6} \frac{0}{7} \| \frac{1}{4} \frac{1}{9} \frac{9}{7} \frac{2}{2} \frac{7}{1}$ \\
\hline 203 & 2 & $A_{1}$ & $2 \underline{A_{1}}+\underline{A_{3}}$ & 4 & 8 & $\frac{0}{0} \frac{0}{2} \frac{0}{1} \frac{0}{3} \frac{0}{4} \frac{0}{5} \frac{0}{6} \frac{0}{7} \| \frac{1}{4} \frac{1}{7} \frac{5}{7} \frac{6}{5} \frac{6}{4}$ \\
\hline 204 & 2 & $A_{1}$ & $5 \underline{A_{1}}$ & 6 & 12 & $\frac{0}{0} \frac{0}{2} \frac{0}{1} \frac{0}{3} \frac{0}{4} \frac{0}{5} \frac{0}{6} \frac{0}{7} \| \frac{1}{7} \frac{5}{6} \frac{2}{4} \frac{6}{4} \frac{0}{a}$ \\
\hline 205 & 2 & $A_{1}$ & $\underline{A_{1}}+\underline{D_{4}}$ & 3 & 6 & $\frac{0}{0} \frac{0}{2} \frac{0}{1} \frac{0}{3} \frac{0}{4} \frac{0}{5} \frac{0}{6} \frac{0}{7} \| \frac{1}{4} \frac{4}{7} \frac{1}{7} \frac{2}{2} \frac{0}{a}$ \\
\hline 206 & 2 & $A_{1}$ & $\underline{A_{2}}+\underline{A_{3}}$ & 4 & 6 & $\frac{0}{0} \frac{0}{2} \frac{0}{1} \frac{0}{3} \frac{0}{4} \frac{0}{5} \frac{0}{7} \| 1 \frac{1}{4} \frac{15}{7} \frac{5}{9} \frac{6}{4}$ \\
\hline 207 & 2 & $A_{1}$ & $\underline{A_{5}}$ & 4 & 3 & $\frac{0}{0} \frac{0}{2} \frac{0}{1} \frac{0}{3} \frac{0}{4} \frac{0}{5} \frac{0}{6} \frac{0}{7} \| \frac{1}{1} \frac{1}{4} \frac{1}{7} \frac{9}{7} \frac{2}{2}$ \\
\hline 208 & 2 & $A_{1}$ & $\underline{A_{5}}$ & 3 & 3 & $\frac{0}{7} \| \frac{1}{1} \frac{4}{2} \frac{15}{7} \frac{2}{6}$ \\
\hline 209 & 2 & $A_{1}$ & $\underline{D_{5}}$ & 2 & 3 & $\frac{0}{0} \frac{0}{2} \frac{0}{1} \frac{0}{3} \frac{0}{4} \frac{0}{5} \frac{0}{6} \frac{0}{7} \| \frac{1}{1} \frac{1}{4} \frac{4}{7} \frac{1}{7} \frac{2}{2}$ \\
\hline 210 & 2 & $A_{1}$ & $2 \underline{A_{1}}+\underline{D_{4}}$ & 2 & 4 & $\frac{0}{0} \frac{0}{2} \frac{0}{1} \frac{0}{3} \frac{0}{4} \frac{0}{5} \frac{0}{6} \frac{0}{7} \| \frac{1}{1} \frac{1}{4} \frac{1}{7} \frac{9}{7} \frac{5}{9} \frac{6}{4}$ \\
\hline 211 & 2 & $A_{1}$ & $2 \underline{A_{3}}$ & 2 & 3 & $\frac{0}{0} \frac{0}{2} \frac{0}{1} \frac{0}{3} \frac{0}{4} \frac{0}{5} \frac{0}{6}\left\|_{7}\right\| \frac{1}{1} \frac{4}{2} \frac{1}{7} \frac{5}{6} \frac{2}{4} \frac{6}{4}$ \\
\hline 212 & 2 & $A_{1}$ & $6 \underline{A_{1}}$ & 4 & 9 & $\frac{0}{0} \frac{0}{2} \frac{0}{1} \frac{0}{3} \frac{0}{4} \frac{0}{5} \frac{0}{6}\left\|_{7}\right\| \frac{1}{4} \frac{1}{9} \frac{9}{7} \frac{5}{9} \frac{7}{8} \frac{7}{1}$ \\
\hline 213 & 2 & $A_{1}$ & $\underline{D_{6}}$ & 1 & 1 & $\frac{0}{0} \frac{0}{2} \frac{0}{1} \frac{0}{3} \frac{0}{4} \frac{0}{5} \frac{0}{6} \frac{0}{7} \| \frac{3}{7} \frac{1}{1} \frac{1}{4} \frac{4}{7} \frac{1}{7} \frac{2}{2}$ \\
\hline 214 & 2 & $2 A_{1}$ & $A_{0}$ & 16 & 26 & $\frac{0}{0} \frac{0}{2} \frac{0}{1} \frac{0}{4} \frac{0}{3} \frac{0}{5} \frac{0}{7} \|$ \\
\hline 215 & 2 & $2 A_{1}$ & $\underline{A_{1}}$ & 12 & 17 & $\frac{00}{0} \frac{0}{2} \frac{0}{1} \frac{0}{4} \frac{0}{5} \frac{0}{6} \frac{0}{7} \| \frac{2}{2}$ \\
\hline 216 & 2 & $2 A_{1}$ & $\underline{A_{1}}$ & 8 & 25 & $\frac{0}{0} \frac{0}{2} \frac{0}{1} \frac{0}{4} \frac{0}{3} \frac{0}{5} \frac{0}{7} \| \frac{7}{3}$ \\
\hline 217 & 2 & $2 A_{1}$ & $2 A_{1}$ & 14 & 20 & $\frac{0}{5} \frac{0}{6} \frac{0}{7} \| \frac{6}{0} \frac{6}{3}$ \\
\hline 218 & 2 & $2 A_{1}$ & $2 \underline{A_{1}}$ & 10 & 12 & $\frac{0}{0} \frac{0}{2} \frac{0}{1} \frac{0}{4} \frac{0}{3} \frac{0}{5} \frac{0}{6}\|\|_{2} \frac{2}{9}$ \\
\hline 219 & 2 & $2 A_{1}$ & $2 \underline{A_{1}}$ & 8 & 12 & $\frac{0}{0} \frac{0}{2} \frac{0}{1} \frac{0}{4} \frac{0}{3} \frac{0}{5} \frac{0}{6} \| \mid \frac{1}{7} \frac{5}{9}$ \\
\hline 220 & 2 & $2 A_{1}$ & $2 \underline{A_{1}}$ & 6 & 16 & $\frac{0}{0} \frac{0}{2} \frac{0}{1} \frac{0}{4} \frac{0}{3} \frac{0}{5} \frac{0}{6} \|^{7} \mid \frac{2}{2} \frac{7}{3}$ \\
\hline 221 & 2 & $2 A_{1}$ & $\underline{A_{2}}$ & 8 & 9 & $\frac{0}{0} \frac{0}{2} \frac{0}{1} \frac{0}{4} \frac{0}{3} \frac{0}{5} \frac{0}{6} \frac{0}{7} \| \frac{1}{7} \frac{2}{2}$ \\
\hline 222 & 2 & $2 A_{1}$ & $2 A_{1}+\underline{A_{1}}$ & 10 & 13 & $\frac{0}{0} \frac{0}{2} \frac{0}{1} \frac{0}{4} \frac{0}{3} \frac{0}{5} \frac{0}{6}\|\|_{7} \frac{1}{0} \frac{6}{3}$ \\
\hline 223 & 2 & $2 A_{1}$ & $2 A_{1}+\underline{A_{1}}$ & 7 & 19 & $\frac{0}{0} \frac{0}{2} \frac{000}{1} \frac{0}{3} \frac{0}{5} \frac{0}{6}|| \frac{7}{3} \frac{6}{3} \frac{6}{3}$ \\
\hline 224 & 2 & $2 A_{1}$ & $3 \underline{A_{1}}$ & 6 & 9 & $\frac{0}{0} \frac{0}{2} \frac{0}{1} \frac{0}{4} \frac{0}{3} \frac{0}{5} \frac{0}{7}\|\|_{7} \frac{15}{9} \frac{6}{4}$ \\
\hline 225 & 2 & $2 A_{1}$ & $3 \underline{A_{1}}$ & 5 & 11 & $\frac{0}{0} \frac{0}{2} \frac{0}{1} \frac{0}{4} \frac{0}{3} \frac{0}{5} \frac{0}{7}\|\|_{2} \frac{2}{3} \frac{9}{9}$ \\
\hline 226 & 2 & $2 A_{1}$ & $3 \underline{A_{1}}$ & 4 & 11 & $\frac{0}{0} \frac{0}{2} \frac{000000}{1} \frac{0}{3} \frac{0}{6} \| \frac{15}{7} \frac{7}{3}$ \\
\hline 227 & 2 & $2 A_{1}$ & $\underline{A_{1}}+\underline{A_{2}}$ & 4 & 8 & $\frac{0}{0} \frac{0}{2} \frac{0}{1} \frac{0}{4} \frac{0}{3} \frac{0}{5} \frac{0}{6}\|\| \frac{1}{7} \frac{2}{2} \frac{7}{3}$ \\
\hline 228 & 2 & $2 A_{1}$ & $A_{3}$ & 10 & 11 & $\frac{0}{0} \frac{0}{2} \frac{0}{1} \frac{0}{4} \frac{0}{3} \frac{0}{5} \frac{0}{6} \frac{0}{7} \| \frac{5}{3} \frac{5}{2}$ \\
\hline 229 & 2 & $2 A_{1}$ & $\underline{A_{3}}$ & 6 & 5 & $\frac{0}{0} \frac{0}{2} \frac{000}{1} \frac{0}{3} \frac{0}{5} \frac{0}{6}\left\|_{7}\right\| \frac{19}{7} \frac{2}{2}$ \\
\hline 230 & 2 & $2 A_{1}$ & $\underline{A_{3}}$ & 4 & 5 & $\frac{0}{0} \frac{0}{2} \frac{0}{1} \frac{0}{4} \frac{0}{3} \frac{0}{5} \frac{0}{6}\|\| \frac{4}{7} \frac{1}{7} \frac{2}{2}$ \\
\hline 231 & 2 & $2 A_{1}$ & $2 A_{1}+\underline{A_{2}}$ & 6 & 7 & $\frac{0}{0} \frac{0}{2} \frac{0}{1} \frac{0}{4} \frac{0}{3} \frac{0}{5} \frac{0}{6}\left\|^{7}\right\| \frac{4}{2} \frac{16}{7} \frac{6}{3}$ \\
\hline 232 & 2 & $2 A_{1}$ & $4 A_{1}$ & 12 & 15 & $\frac{00}{0} \frac{0}{2} \frac{0}{1} \frac{0}{3} \frac{0}{5} \frac{0}{6} \frac{0}{7}|| \frac{5}{0} \frac{6}{3} \frac{6}{1}$ \\
\hline 233 & 2 & $2 A_{1}$ & $2 \underline{A_{1}}+2 A_{1}$ & 8 & 9 & $\frac{0}{0} \frac{0}{2} \frac{0}{1} \frac{0}{4} \frac{0}{3} \frac{0}{6} \frac{0}{7} \| \mid \frac{5}{0} \frac{2}{3} \frac{2}{3}$ \\
\hline 234 & 2 & $2 A_{1}$ & $2 A_{1}+2 \underline{A_{1}}$ & 5 & 12 & $\frac{0}{0} \frac{0}{2} \frac{0}{1} \frac{0}{4} \frac{0}{3} \frac{0}{5} \frac{0}{6}\|\| \frac{1}{7} \frac{7}{3} \frac{6}{0}$ \\
\hline 235 & 2 & $2 A_{1}$ & $4 \underline{A_{1}}$ & 4 & 7 & $\frac{00}{0} \frac{0}{2} \frac{0}{1} \frac{0}{3} \frac{00}{5} \frac{0}{7} \| \mid \frac{19}{7} \frac{5}{7} \frac{6}{4}$ \\
\hline 236 & 2 & $2 A_{1}$ & $4 \underline{A_{1}}$ & 3 & 8 & $\frac{00}{0} \frac{0}{2} \frac{0}{1} \frac{0}{4} \frac{0}{5} \frac{0}{6} \frac{0}{7} \| \frac{1}{7} \frac{5}{9} \frac{7}{4}$ \\
\hline 237 & 2 & $2 A_{1}$ & $\underline{A_{1}}+\ldots$ & 6 & 8 & $\frac{0}{5} \frac{0}{7} \| \frac{4}{2} \frac{5}{0} \frac{5}{3} \frac{2}{2}$ \\
\hline
\end{tabular}

\begin{tabular}{|c|c|c|c|c|c|c|}
\hline & $\mathrm{d}$ & $D(A)$ & $D(B)$ & $\# E$ & $\# G$ & $\sigma_{A} \| B$ \\
\hline 238 & 2 & $2 A_{1}$ & $\underline{A_{1}}+A_{3}$ & 5 & 10 & $\frac{0}{0} \frac{0}{2} \frac{0}{1} \frac{0}{4} \frac{0}{3} \frac{0}{6} \frac{0}{7}\|\|_{0} \frac{5}{3} \frac{2}{2} \frac{7}{3}$ \\
\hline 239 & 2 & $2 A_{1}$ & $\underline{A_{1}}+\underline{A_{3}}$ & 3 & 4 & $\frac{0}{0} \frac{0}{2} \frac{0}{1} \frac{0}{4} \frac{000}{5} \frac{0}{6}\left\|_{7}\right\| \frac{1}{7} \frac{2}{7} \frac{7}{3}$ \\
\hline 240 & 2 & $2 A_{1}$ & $\underline{A_{1}}+\underline{A_{3}}$ & 2 & 4 & $\frac{0}{0} \frac{0}{2} \frac{0}{1} \frac{0}{4} \frac{000}{3} \frac{0}{6} \frac{0}{7} \| \frac{4}{2} \frac{12}{7} \frac{7}{3}$ \\
\hline 241 & 2 & $2 A_{1}$ & $D_{4}$ & 6 & 5 & $\frac{0000000000}{0} \frac{0}{1} \frac{0}{4} \frac{0}{5} \frac{4}{7} \mid \frac{4}{6} \frac{12}{7} \frac{2}{2}$ \\
\hline 242 & 2 & $2 A_{1}$ & $\underline{D_{4}}$ & 2 & 3 & $\frac{0}{0} \frac{0}{2} \frac{0}{1} \frac{0}{4} \frac{0}{3} \frac{00}{6} \frac{0}{7} \| \mid \frac{4}{2} \frac{4}{7} \frac{2}{7}$ \\
\hline 243 & 2 & $2 A_{1}$ & $2 A_{1}+A_{3}$ & 8 & 8 & $\frac{0}{0} \frac{0}{2} \frac{0}{1} \frac{0}{4} \frac{0}{3} \frac{0}{5} \frac{0}{6} \frac{0}{7} \| \frac{4}{3} \frac{4}{6} \frac{16}{7} \frac{6}{2}$ \\
\hline 244 & 2 & $2 A_{1}$ & $2 \underline{A_{1}}+A_{3}$ & 4 & 6 & $\frac{0}{0} \frac{0}{2} \frac{0}{1} \frac{0}{4} \frac{0}{3} \frac{0}{5} \frac{0}{6} \frac{0}{7} \| \frac{4}{3} \frac{4}{6} \frac{15}{9} \frac{6}{4}$ \\
\hline 245 & 2 & $2 A_{1}$ & $2 A_{1}+\underline{A_{3}}$ & 4 & 4 & $\frac{0}{0} \frac{0}{2} \frac{0}{1} \frac{0}{4} \frac{0}{3} \frac{00}{5} \frac{0}{7} \|^{7} \mid \frac{4}{2} \frac{4}{7} \frac{16}{7} \frac{6}{3}$ \\
\hline 246 & 2 & $2 A_{1}$ & $2 \underline{A_{1}}+A_{3}$ & 3 & 7 & $\frac{0}{0} \frac{0}{2} \frac{0}{1} \frac{0}{4} \frac{0}{3} \frac{0}{5} \frac{0}{6} \frac{0}{7} \| \frac{4}{2} \frac{5}{3} \frac{5}{2} \frac{7}{3}$ \\
\hline 247 & 2 & $2 A_{1}$ & $2 A_{1}+\underline{A_{1}}+\underline{A_{2}}$ & 3 & 6 & $\frac{0}{0} \frac{0}{2} \frac{0}{1} \frac{0}{4} \frac{0}{3} \frac{0}{5} \frac{0}{6} \frac{0}{7} \| \frac{4}{2} \frac{1}{7} \frac{7}{3} \frac{6}{3} \frac{6}{3}$ \\
\hline 248 & 2 & $2 A_{1}$ & $4 A_{1}+\underline{A_{1}}$ & 8 & 10 & $\frac{0}{0} \frac{0}{2} \frac{0}{1} \frac{0}{4} \frac{0}{3} \frac{0}{5} \frac{0}{6} \frac{0}{7} \| \frac{4}{2} \frac{5}{3} \frac{5}{3} \frac{6}{2}$ \\
\hline 249 & 2 & $2 A_{1}$ & $4 A_{1}+\underline{A_{1}}$ & 6 & 14 & $\left.\frac{0}{0} \frac{0}{2} \frac{0}{1} \frac{0}{4} \frac{0}{3} \frac{0}{5} \frac{0}{6}\right) \mid 5 \frac{5}{7} \frac{7}{3} \frac{66}{1} \frac{6}{2}$ \\
\hline 250 & 2 & $2 A_{1}$ & $3 \underline{A_{1}}+2 A_{1}$ & 4 & 8 & $\frac{0}{0} \frac{0}{2} \frac{0}{1} \frac{0}{4} \frac{0}{3} \frac{0}{5} \frac{0}{6} \frac{0}{7} \| \frac{5}{0} \frac{5}{3} \frac{7}{3} \frac{9}{8}$ \\
\hline 251 & 2 & $2 A_{1}$ & $5 \underline{A_{1}}$ & 2 & 6 & $\frac{0}{0} \frac{0}{2} \frac{0}{1} \frac{0}{4} \frac{0}{3} \frac{0}{5} \frac{0}{6} \frac{0}{7} \| \frac{19}{7} \frac{9}{7} \frac{6}{4} \frac{7}{3}$ \\
\hline 252 & 2 & $2 A_{1}$ & $\underline{A_{1}}+D_{4}$ & 3 & 4 & $\frac{0}{0} \frac{0}{2} \frac{0}{1} \frac{0}{4} \frac{00}{3} \frac{0}{5} \frac{0}{7}\|\|_{3} \frac{4}{6} \frac{1}{7} \frac{2}{2} \frac{7}{3}$ \\
\hline 253 & 2 & $2 A_{1}$ & $\underline{A_{1}}+\underline{D_{4}}$ & 1 & 2 & $\frac{0}{0} \frac{0}{2} \frac{0}{1} \frac{0}{4} \frac{0}{3} \frac{0}{5} \frac{0}{6} \frac{0}{7} \| \frac{4}{2} \frac{4}{7} \frac{1}{7} \frac{2}{2} \frac{7}{3}$ \\
\hline 254 & 2 & $2 A_{1}$ & $D_{5}$ & 2 & 3 & $\frac{0}{0} \frac{0}{2} \frac{0}{1} \frac{0}{4} \frac{0}{3} \frac{0}{5} \frac{0}{6} \frac{0}{7} \| 1 \frac{1}{3} \frac{14}{2} \frac{1}{7} \frac{2}{2}$ \\
\hline 255 & 2 & $2 A_{1}$ & $2 A_{1}+D_{4}$ & 4 & 4 & $\frac{0}{0} \frac{0}{2} \frac{0}{1} \frac{0}{4} \frac{0}{3} \frac{0}{5} \frac{0}{6} \frac{0}{7} \| \frac{1}{0} \frac{1}{3} \frac{1}{2} \frac{6}{7} \frac{6}{1}$ \\
\hline 256 & 2 & $2 A_{1}$ & $2 A_{3}$ & 4 & 5 & $\frac{0}{0} \frac{0}{2} \frac{0000000}{1} \frac{0}{3} \frac{0}{6}\|\|_{0} \frac{1}{3} \frac{4}{2} \frac{5}{1} \frac{2}{2}$ \\
\hline 257 & 2 & $2 A_{1}$ & $2 A_{1}+\underline{A_{1}}+A_{3}$ & 4 & 7 & $\frac{0}{0} \frac{0}{2} \frac{0000000}{1} \frac{0}{3} \frac{0}{5}\left\|_{7}\right\| \frac{4}{3} \frac{4}{7} \frac{7}{3} \frac{6}{1} \frac{6}{2}$ \\
\hline 258 & 2 & $2 A_{1}$ & $3 \underline{A_{1}}+A_{3}$ & 2 & 5 & $\frac{0}{0} \frac{0}{2} \frac{00}{1} \frac{0}{4} \frac{0}{3} \frac{0}{5} \frac{0}{7}\|\|_{3} \frac{4}{6} \frac{4}{7} \frac{5}{9} \frac{6}{4}$ \\
\hline 259 & 2 & $2 A_{1}$ & $2 A_{1}+\underline{A_{1}}+\underline{A_{3}}$ & 2 & 3 & $\frac{0}{0} \frac{0}{2} \frac{0000000}{1} \frac{0}{3} \frac{0}{5} \frac{4}{7} \| \frac{4}{2} \frac{4}{7} \frac{7}{7} \frac{6}{0} \frac{6}{3}$ \\
\hline 260 & 2 & $2 A_{1}$ & $2 \underline{A_{1}}+4 A_{1}$ & 6 & 7 & $\frac{0}{0} \frac{0}{2} \frac{0}{1} \frac{0}{4} \frac{0}{3} \frac{0}{5} \frac{0}{6} \frac{0}{7} \| \frac{4}{3} \frac{45}{1} \frac{5}{2} \frac{5}{9} \frac{6}{4}$ \\
\hline 261 & 2 & $2 A_{1}$ & $4 A_{1}+2 \underline{A_{1}}$ & 4 & 9 & $\frac{0}{0} \frac{0}{2} \frac{000}{1} \frac{0}{3} \frac{0}{5} \frac{0}{6}\left\|_{7}\right\| \frac{4}{2} \frac{5}{3} \frac{7}{3} \frac{66}{1} \frac{6}{2}$ \\
\hline 262 & 2 & $2 A_{1}$ & $\underline{A_{1}}+D_{5}$ & 1 & 2 & $\frac{0}{0} \frac{0}{2} \frac{00}{1} \frac{0}{4} \frac{0}{3} \frac{0}{6} \frac{0}{7}\|\|_{0} \frac{1}{3} \frac{4}{2} \frac{12}{7} \frac{7}{3}$ \\
\hline 263 & 2 & $2 A_{1}$ & $2 A_{1}+\underline{A_{1}}+D_{4}$ & 2 & 3 & $\frac{0}{0} \frac{0}{2} \frac{0000000}{1} \frac{0}{3} \frac{0}{5} \frac{1}{7} \| \frac{1}{3} \frac{1}{2} \frac{1}{7} \frac{7}{3} \frac{6}{1} \frac{6}{2}$ \\
\hline 264 & 2 & $2 A_{1}$ & $3 \underline{A_{1}}+4 A_{1}$ & 3 & 6 & $\frac{0}{6} \frac{0}{7} \| \frac{44}{3} \frac{45}{1} \frac{5}{2} \frac{6}{9} \frac{7}{3}$ \\
\hline 265 & 2 & $2 A_{1}$ & $\underline{A_{1}}+2 A_{3}$ & 2 & 4 & $\frac{0}{0} \frac{0}{2} \frac{0}{1} \frac{0}{4} \frac{0}{3} \frac{0}{5} \frac{0}{6}\left\|_{7}\right\| \frac{1}{0} \frac{1}{3} \frac{4}{2} \frac{5}{2} \frac{2}{2} \frac{7}{3}$ \\
\hline 266 & 2 & $3 A_{1}$ & $A_{0}$ & 8 & 8 & $\frac{0}{0} \frac{0}{2} \frac{0}{1} \frac{0}{4} \frac{000}{6} \frac{0}{5} \|$ \\
\hline 267 & 2 & $3 A_{1}$ & $\underline{A_{1}}$ & 8 & 7 & $\frac{0}{0} \frac{0}{2} \frac{0}{1}$ \\
\hline 268 & 2 & $3 A_{1}$ & $\underline{A_{1}}$ & 4 & 7 & $\frac{0000000000}{0} \frac{0}{1} \frac{0}{4} \frac{5}{6} \|^{\frac{5}{7}}$ \\
\hline 269 & 2 & $3 A_{1}$ & $2 A_{1}$ & 6 & 6 & $\frac{0}{0} \frac{0}{2} \frac{0}{1} \frac{0}{4} \frac{0}{3} \frac{0}{6} \frac{0}{5} \| \frac{6}{7} \frac{6}{3}$ \\
\hline 270 & 2 & $3 A_{1}$ & $2 \underline{A_{1}}$ & 4 & 6 & $\frac{0}{0} \frac{0}{2} \frac{0}{1} \frac{0}{4} \frac{0}{3} \frac{0}{6} \frac{0}{5} \frac{1}{7} \| \frac{9}{7} \frac{5}{9}$ \\
\hline 271 & 2 & $3 A_{1}$ & $2 \underline{A_{1}}$ & 2 & 6 & $\frac{0}{6} \frac{0}{5} \frac{0}{7} \| \frac{5}{9} \frac{6}{4}$ \\
\hline 272 & 2 & $3 A_{1}$ & $A_{2}$ & 8 & 7 & $\frac{0}{0} \frac{0}{2} \frac{0}{1} \frac{0}{4} \frac{0}{3} \frac{0}{6} \frac{0}{5} \frac{1}{7} \| \frac{4}{3} \frac{5}{3}$ \\
\hline 273 & 2 & $3 A_{1}$ & $2 A_{1}+\underline{A_{1}}$ & 6 & 5 & $\frac{0}{0} \frac{0}{2} \frac{0}{1} \frac{0}{4} \frac{0}{3} \frac{0}{6} \frac{0}{7} \| \frac{9}{7} \frac{6}{0} \frac{6}{3}$ \\
\hline 274 & 2 & $3 A_{1}$ & $2 A_{1}+\underline{A_{1}}$ & 3 & 5 & $\frac{0}{0} \frac{0}{2} \frac{0}{1} \frac{0}{4} \frac{0}{3} \frac{0}{6} \frac{0}{7} \| \frac{56}{7} \frac{7}{2}$ \\
\hline 275 & 2 & $3 A_{1}$ & $3 \underline{A_{1}}$ & 2 & 5 & $\frac{0}{0} \frac{0}{2} \frac{0}{1} \frac{0}{4} \frac{0}{3} \frac{0}{6} \frac{0}{7} \| \frac{9}{7} \frac{5}{9} \frac{6}{4}$ \\
\hline 276 & 2 & $3 A_{1}$ & $3 \underline{A_{1}}$ & 1 & 5 & $\frac{0}{0} \frac{0}{2} \frac{0}{1} \frac{0}{4} \frac{0}{3} \frac{0}{6} \frac{0}{7}\|\| \frac{5}{9} \frac{6}{4}$ \\
\hline 277 & 2 & $3 A_{1}$ & $\underline{A_{1}}+A_{2}$ & 4 & 6 & $\frac{0}{0} \frac{0}{2} \frac{0}{1} \frac{0}{4} \frac{0}{3} \frac{0}{6} \frac{0}{5} \| \frac{4}{7} \frac{5}{3} \frac{5}{9}$ \\
\hline 278 & 2 & $3 A_{1}$ & $A_{3}$ & 2 & 5 & $\frac{0}{0} \frac{0}{2} \frac{0}{1} \frac{0}{4} \frac{0}{3} \frac{0}{6} \frac{0}{5}\|\| \frac{1}{7} \frac{1}{3} \frac{5}{9}$ \\
\hline 279 & 2 & $3 A_{1}$ & $2 A_{1}+A_{2}$ & 6 & 5 & $\frac{0}{0} \frac{0}{2} \frac{0}{1} \frac{0}{4} \frac{0000}{3} \frac{0}{5} \|_{7} \mid \frac{4}{3} \frac{6}{3} \frac{6}{1} \frac{6}{2}$ \\
\hline 280 & 2 & $3 A_{1}$ & $2 \underline{A_{1}}+A_{2}$ & 2 & 5 & $\frac{0}{0} \frac{0}{2} \frac{0}{1} \frac{0}{4} \frac{0}{3} \frac{00}{6} \frac{0}{7}\|\|_{3} \frac{45}{3} \frac{5}{9}$ \\
\hline 281 & 2 & $3 A_{1}$ & $2 A_{2}$ & 4 & 4 & $\frac{0}{0} \frac{0}{2} \frac{0}{1} \frac{0}{4} \frac{0}{3} \frac{0}{5} \frac{0}{5}\|\|_{0} \frac{1}{3} \frac{6}{5} \frac{7}{0}$ \\
\hline 282 & 2 & $3 A_{1}$ & $4 A_{1}$ & 4 & 5 & $\frac{0}{0} \frac{0}{2} \frac{0}{1} \frac{0}{4} \frac{00}{3} \frac{0}{6} \frac{0}{7} \| \frac{1}{0} \frac{16}{3} \frac{6}{1}$ \\
\hline 283 & 2 & $3 A_{1}$ & $2 A_{1}+2 \underline{A_{1}}$ & 3 & 4 & $\frac{0000000000}{0} \frac{0}{1} 1 \frac{9}{3} \frac{9}{6} \frac{5}{5} \frac{6}{7} \frac{7}{2}$ \\
\hline 284 & 2 & $3 A_{1}$ & $4 \underline{A_{1}}$ & 1 & 4 & $\left.\frac{0}{0} \frac{0}{2} \frac{0}{1} \frac{0}{4} \frac{0}{3} \frac{0}{6} \frac{0}{5}\right) \| \frac{9}{7} \frac{5}{9} \frac{7}{4}$ \\
\hline 285 & 2 & $3 A_{1}$ & $\underline{A_{1}}+A_{3}$ & 2 & 4 & $\frac{0}{0} \frac{0}{2} \frac{0}{1} \frac{0000}{4} \frac{0}{6} \frac{0}{7} \| \frac{9}{7} \frac{1}{0} \frac{15}{9}$ \\
\hline 286 & 2 & $3 A_{1}$ & $\underline{A_{1}}+A_{3}$ & 1 & 4 & $\frac{0000000000}{0} \frac{0}{1} \frac{1}{4} \frac{1}{6} \frac{1}{5} \frac{15}{3} \frac{7}{3}$ \\
\hline 287 & 2 & $3 A_{1}$ & $2 \underline{A_{1}}+A_{3}$ & 1 & 3 & $\frac{0}{0} \frac{0}{2} \frac{0}{1} \frac{0}{4} \frac{0}{3} \frac{0}{6} \frac{0}{5} \frac{0}{7} \| \frac{9}{7} \frac{1}{3} \frac{1}{3} \frac{7}{3}$ \\
\hline 288 & 2 & $3 A_{1}$ & $2 A_{1}+\underline{A_{1}}+\underline{A_{2}}$ & 3 & 4 & $\frac{0}{0} \frac{0}{2} \frac{0}{1} \frac{0}{4} \frac{0}{3} \frac{0}{6} \frac{0}{5} \frac{0}{7} \| \frac{4}{3} \frac{5}{3} \frac{5}{9} \frac{7}{1}$ \\
\hline 289 & 2 & $3 A_{1}$ & $3 \underline{A_{1}}+A_{2}$ & 1 & 4 & $\frac{0}{0} \frac{0}{2} \frac{0}{1} \frac{0}{4} \frac{0}{3} \frac{0}{6} \frac{0}{5} \frac{0}{7} \| \frac{4}{3} \frac{5}{3} \frac{5}{4} \frac{7}{3}$ \\
\hline 290 & 2 & $3 A_{1}$ & $4 A_{1}+\underline{A_{1}}$ & 4 & 4 & $\frac{0}{0} \frac{0}{2} \frac{0}{1} \frac{0}{4} \frac{0}{3} \frac{0}{6} \frac{0}{5} \frac{0}{7} \| \frac{9}{7} \frac{1}{3} \frac{16}{1} \frac{6}{2}$ \\
\hline 291 & 2 & $3 A_{1}$ & $4 A_{1}+\underline{A_{1}}$ & 2 & 4 & $\frac{0}{0} \frac{0}{2} \frac{0}{1} \frac{0}{4} \frac{0}{3} \frac{0}{6} \frac{0}{5} \frac{0}{7} \| \frac{1}{0} \frac{1}{3} \frac{76}{3} \frac{6}{2}$ \\
\hline 292 & 2 & $3 A_{1}$ & $\underline{A_{1}}+2 A_{2}$ & 4 & 3 & $\frac{0}{0} \frac{0}{2} \frac{0}{1} \frac{0}{4} \frac{0}{3} \frac{0}{6} \frac{0}{7}\|\|_{7} \frac{1}{0} \frac{1}{3} \frac{6}{5} \frac{7}{0}$ \\
\hline 293 & 2 & $3 A_{1}$ & $A_{2}+A_{3}$ & 2 & 4 & $\frac{0}{0} \frac{0}{2} \frac{0}{1} \frac{0}{4} \frac{0}{3} \frac{0}{6} \frac{0}{5} \frac{0}{7} \| 1 \frac{1}{0} \frac{1}{3} \frac{45}{2} \frac{5}{9}$ \\
\hline
\end{tabular}




\begin{tabular}{|c|c|c|c|c|c|c|}
\hline & $\mathrm{d}$ & $D(A)$ & $D(B)$ & $\# E$ & $\# G$ & $\sigma_{A} \| B$ \\
\hline 294 & 2 & $3 A_{1}$ & $A_{5}$ & 1 & 3 & $\frac{0}{0} \frac{0}{2} \frac{0}{1} \frac{0}{4} \frac{0}{3} \frac{0}{6} \frac{0}{5} \frac{0}{7} \| 1 \frac{1}{0} \frac{1}{3} \frac{1}{4} \frac{1}{9}$ \\
\hline 295 & 2 & $3 A_{1}$ & $2 A_{3}$ & 2 & 3 & $\frac{0}{0} \frac{0}{2} \frac{0}{1} \frac{0}{4} \frac{0}{3} \frac{0}{6} \frac{0}{5} \frac{0}{7} \| \frac{1}{0} \frac{1}{3} \frac{1}{4} \frac{1}{9} \frac{6}{1} \frac{6}{2}$ \\
\hline 296 & 2 & $3 A_{1}$ & $A_{2}+2 A_{2}$ & 4 & 3 & $\frac{0}{0} \frac{0}{2} \frac{0}{1} \frac{0}{4} \frac{0}{3} \frac{0}{6} \frac{0}{5} \frac{0}{7} \| \frac{1}{0} \frac{1}{3} \frac{4}{4} \frac{5}{2} \frac{6}{5} \frac{7}{0}$ \\
\hline 297 & 2 & $3 A_{1}$ & $4 A_{1}+2 \underline{A_{1}}$ & 2 & 3 & $\frac{0}{0} \frac{0}{2} \frac{0}{1} \frac{0}{4} \frac{0}{3} \frac{0}{6} \frac{0}{5} \frac{0}{7} \| \frac{9}{7} \frac{1}{0} \frac{1}{3} \frac{7}{3} \frac{6}{1} \frac{6}{2}$ \\
\hline 298 & 2 & $3 A_{1}$ & $\underline{A_{1}}+\ldots A_{2}+\ldots A_{3}$ & 1 & 3 & $\frac{0}{0} \frac{0}{2} \frac{0}{1} \frac{0}{4} \frac{0}{3} \frac{0}{6} \frac{0}{5} \frac{0}{7} \| \frac{1}{0} \frac{1}{3} \frac{4}{4} \frac{5}{2} \frac{5}{9} \frac{7}{3}$ \\
\hline 299 & 2 & $3 A_{1}$ & $\underline{A_{1}}+A_{5}$ & 1 & 2 & $\frac{0}{0} \frac{0}{2} \frac{0}{1} \frac{0}{4} \frac{0}{3} \frac{0}{6} \frac{0}{5} \frac{0}{7} \| \frac{9}{7} \frac{1}{0} \frac{1}{3} \frac{1}{4} \frac{1}{9} \frac{5}{9}$ \\
\hline 300 & 2 & $3 A_{1}$ & $\underline{A_{1}}+2 A_{3}$ & 2 & 2 & $\frac{0}{0} \frac{0}{2} \frac{0}{1} \frac{0}{4} \frac{0}{3} \frac{0}{6} \frac{0}{5} \frac{0}{7} \| \frac{9}{7} \frac{1}{0} \frac{1}{3} \frac{1}{4} \frac{1}{9} \frac{6}{1} \frac{6}{2}$ \\
\hline 301 & 2 & $3 A_{1}$ & $A_{2}+A_{5}$ & 1 & 2 & $\frac{0}{0} \frac{0}{2} \frac{0}{1} \frac{0}{4} \frac{0}{3} \frac{0}{6} \frac{0}{5} \frac{0}{7} \| \frac{1}{0} \frac{1}{3} \frac{1}{4} \frac{1}{9} \frac{4}{5} \frac{5}{1} \frac{5}{9}$ \\
\hline 302 & 2 & $3 A_{1}^{\prime}$ & $A_{0}$ & 0 & 24 & $\frac{9}{5} \frac{3}{6} \frac{3}{5} \frac{3}{4} \frac{0}{5} \frac{0}{4} \frac{0}{7} \frac{0}{6} \|$ \\
\hline 303 & 2 & $3 A_{1}^{\prime}$ & $\underline{A_{1}}$ & 0 & 15 & $\frac{9}{5} \frac{3}{6} \frac{3}{5} \frac{3}{4} \frac{0}{5} \frac{0}{4} \frac{0}{7} \frac{0}{6} \| \frac{0}{9}$ \\
\hline 304 & 2 & $3 A_{1}^{\prime}$ & $2 A_{1}$ & 0 & 18 & $\frac{9}{5} \frac{3}{6} \frac{3}{5} \frac{3}{4} \frac{0}{5} \frac{0}{4} \frac{0}{7} \frac{0}{6} \| \frac{1}{1} \frac{4}{2}$ \\
\hline 305 & 2 & $3 A_{1}^{\prime}$ & $2 \underline{A_{1}}$ & 0 & 10 & $\frac{9}{5} \frac{3}{6} \frac{3}{5} \frac{3}{4} \frac{0}{5} \frac{0}{4} \frac{0}{7} \frac{0}{6} \| \frac{0}{9} \frac{4}{7}$ \\
\hline 306 & 2 & $3 A_{1}^{\prime}$ & $\underline{A_{2}}$ & 0 & 7 & $\frac{9}{5} \frac{3}{6} \frac{3}{5} \frac{3}{4} \frac{0}{5} \frac{0}{4} \frac{0}{7} \frac{0}{6} \| \frac{0}{9} \frac{1}{0}$ \\
\hline 307 & 2 & $3 A_{1}^{\prime}$ & $2 A_{1}+\underline{A_{1}}$ & 0 & 11 & $\frac{9}{5} \frac{3}{6} \frac{3}{5} \frac{3}{4} \frac{0}{5} \frac{0}{4} \frac{0}{7} \frac{0}{6} \| \frac{0}{9} \frac{1}{1} \frac{4}{2}$ \\
\hline 308 & 2 & $3 A_{1}^{\prime}$ & $3 \underline{A_{1}}$ & 0 & 7 & $\frac{9}{5} \frac{3}{6} \frac{3}{5} \frac{3}{4} \frac{0}{5} \frac{0}{4} \frac{0}{7} \frac{0}{6} \| \frac{0}{9} \frac{4}{7} \frac{7}{1}$ \\
\hline 309 & 2 & $3 A_{1}^{\prime}$ & $A_{3}$ & 0 & 9 & $\frac{9}{5} \frac{3}{6} \frac{3}{5} \frac{3}{4} \frac{0}{5} \frac{0}{4} \frac{0}{7} \frac{0}{6} \| \frac{0}{9} \frac{1}{2} \frac{4}{3}$ \\
\hline 310 & 2 & $3 A_{1}^{\prime}$ & $\underline{A_{3}}$ & 0 & 3 & $\frac{9}{5} \frac{3}{6} \frac{3}{5} \frac{3}{4} \frac{0}{5} \frac{0}{4} \frac{0}{7} \frac{0}{6} \| \frac{0}{9} \frac{1}{0} \frac{0}{a}$ \\
\hline 311 & 2 & $3 A_{1}^{\prime}$ & $2 A_{1}+\underline{A_{2}}$ & 0 & 5 & $\frac{9}{5} \frac{3}{6} \frac{3}{5} \frac{3}{4} \frac{0}{5} \frac{0}{4} \frac{0}{7} \frac{0}{6} \| \frac{0}{9} \frac{1}{0} \frac{1}{7} \frac{2}{4}$ \\
\hline 312 & 2 & $3 A_{1}^{\prime}$ & $2 A_{2}$ & 0 & 12 & $\frac{9}{5} \frac{3}{6} \frac{3}{5} \frac{3}{4} \frac{0}{5} \frac{0}{4} \frac{0}{7} \frac{0}{6} \| 1 \frac{1}{1} \frac{1}{7} \frac{2}{4} \frac{4}{2}$ \\
\hline 313 & 2 & $3 A_{1}^{\prime}$ & $4 A_{1}$ & 0 & 13 & $\frac{9}{5} \frac{3}{6} \frac{3}{5} \frac{3}{4} \frac{0}{5} \frac{0}{4} \frac{0}{7} \frac{0}{6} \| \frac{1}{1} \frac{1}{6} \frac{3}{9} \frac{4}{2}$ \\
\hline 314 & 2 & $3 A_{1}^{\prime}$ & $2 A_{1}+2 \underline{A_{1}}$ & 0 & 7 & $\frac{9}{5} \frac{3}{6} \frac{3}{5} \frac{3}{4} \frac{0}{5} \frac{0}{4} \frac{0}{7} \frac{0}{6} \| \frac{0}{9} \frac{4}{7} \frac{1}{1} \frac{4}{2}$ \\
\hline 315 & 2 & $3 A_{1}^{\prime}$ & $4 \underline{A_{1}}$ & 0 & 5 & $\frac{9}{5} \frac{3}{6} \frac{3}{5} \frac{3}{4} \frac{0}{5} \frac{0}{4} \frac{0}{7} \frac{0}{6} \| \mid \frac{0}{9} \frac{4}{7} \frac{7}{1} \frac{0}{a}$ \\
\hline 316 & 2 & $3 A_{1}^{\prime}$ & $\underline{A_{1}}+A_{3}$ & 0 & 6 & $\frac{9}{5} \frac{3}{6} \frac{3}{5} \frac{3}{4} \frac{0}{5} \frac{0}{4} \frac{0}{7} \frac{0}{6} \| \frac{0}{9} \frac{4}{7} \frac{1}{7} \frac{2}{4}$ \\
\hline 317 & 2 & $3 A_{1}^{\prime}$ & $D_{4}$ & 0 & 3 & $\frac{9}{5} \frac{3}{6} \frac{3}{5} \frac{3}{4} \frac{0}{5} \frac{0}{4} \frac{0}{7} \frac{0}{6} \| \frac{0}{9} \frac{1}{0} \frac{1}{1} \frac{4}{2}$ \\
\hline 318 & 2 & $3 A_{1}^{\prime}$ & $\underline{D_{4}}$ & 0 & 1 & $\frac{9}{5} \frac{3}{6} \frac{3}{5} \frac{3}{4} \frac{0}{5} \frac{0}{4} \frac{0}{7} \frac{0}{6} \| \frac{1}{0} \frac{0}{9} \frac{4}{5} \frac{6}{9}$ \\
\hline 319 & 2 & $3 A_{1}^{\prime}$ & $2 A_{1}+A_{3}$ & 0 & 6 & $\frac{9}{5} \frac{3}{6} \frac{3}{5} \frac{3}{4} \frac{0}{5} \frac{0}{4} \frac{0}{7} \frac{0}{6} \| \frac{0}{9} \frac{1}{1} \frac{1}{6} \frac{3}{9} \frac{4}{2}$ \\
\hline 320 & 2 & $3 A_{1}^{\prime}$ & $2 \underline{A_{1}}+A_{3}$ & 0 & 4 & $\frac{9}{5} \frac{3}{6} \frac{3}{5} \frac{3}{4} \frac{0}{5} \frac{0}{4} \frac{0}{7} \frac{0}{6} \| \frac{0}{9} \frac{4}{7} \frac{7}{1} \frac{1}{1} \frac{4}{2}$ \\
\hline 321 & 2 & $3 A_{1}^{\prime}$ & $2 A_{1}+\underline{A_{3}}$ & 0 & 2 & $\frac{9}{5} \frac{3}{6} \frac{3}{5} \frac{3}{4} \frac{0}{5} \frac{0}{4} \frac{0}{7} \frac{0}{6} \| \frac{0}{9} \frac{1}{0} \frac{0}{a} \frac{1}{7} \frac{2}{4}$ \\
\hline 322 & 2 & $3 A_{1}^{\prime}$ & $4 A_{1}+\underline{A_{1}}$ & 0 & 8 & $\frac{9}{5} \frac{3}{6} \frac{3}{5} \frac{3}{4} \frac{0}{5} \frac{0}{4} \frac{0}{7} \frac{0}{6} \| \frac{0}{9} \frac{1}{1} \frac{4}{2} \frac{7}{3} \frac{9}{9}$ \\
\hline 323 & 2 & $3 A_{1}^{\prime}$ & $\underline{A_{1}}+2 A_{2}$ & 0 & 7 & $\frac{9}{5} \frac{3}{6} \frac{3}{5} \frac{3}{4} \frac{0}{5} \frac{0}{4} \frac{0}{7} \frac{0}{6} \| \frac{0}{9} \frac{1}{1} \frac{1}{7} \frac{2}{4} \frac{4}{2}$ \\
\hline 324 & 2 & $3 A_{1}^{\prime}$ & $A_{5}$ & 0 & 5 & $\frac{9}{5} \frac{3}{6} \frac{3}{5} \frac{3}{4} \frac{0}{5} \frac{0}{4} \frac{0}{7} \frac{0}{6} \| \frac{0}{9} \frac{1}{1} \frac{2}{0} \frac{4}{2} \frac{6}{0}$ \\
\hline 325 & 2 & $3 A_{1}^{\prime}$ & $D_{5}$ & 0 & 1 & $\frac{9}{5} \frac{3}{6} \frac{3}{5} \frac{3}{4} \frac{0}{5} \frac{0}{4} \frac{0}{7} \frac{0}{6} \| \frac{1}{0} \frac{0}{9} \frac{4}{5} \frac{1}{7} \frac{2}{4}$ \\
\hline 326 & 2 & $3 A_{1}^{\prime}$ & $2 A_{1}+D_{4}$ & 0 & 2 & $\frac{9}{5} \frac{3}{6} \frac{3}{5} \frac{3}{4} \frac{0}{5} \frac{0}{4} \frac{0}{7} \frac{0}{6} \| \frac{0}{9} \frac{1}{0} \frac{1}{1} \frac{4}{2} \frac{7}{3} \frac{9}{9}$ \\
\hline 327 & 2 & $3 A_{1}^{\prime}$ & $2 A_{3}$ & 0 & 7 & $\frac{9}{5} \frac{3}{6} \frac{3}{5} \frac{3}{4} \frac{0}{5} \frac{0}{4} \frac{0}{7} \frac{0}{6} \| \frac{1}{1} \frac{1}{6} \frac{2}{1} \frac{3}{9} \frac{4}{2} \frac{6}{1}$ \\
\hline 328 & 2 & $3 A_{1}^{\prime}$ & $2 A_{3}$ & 0 & 3 & $\frac{9}{5} \frac{3}{6} \frac{3}{5} \frac{3}{4} \frac{0}{5} \frac{0}{4} \frac{0}{7} \frac{0}{6} \| \frac{0}{9} \frac{4}{7} \frac{1}{7} \frac{2}{4} \frac{5}{5} \frac{6}{2}$ \\
\hline 329 & 2 & $3 A_{1}^{\prime}$ & $2 A_{2}+\underline{A_{2}}$ & 0 & 3 & $\frac{9}{5} \frac{3}{6} \frac{3}{5} \frac{3}{4} \frac{0}{5} \frac{0}{4} \frac{0}{7} \frac{0}{6} \| \frac{0}{9} \frac{1}{0} \frac{1}{7} \frac{2}{4} \frac{5}{7} \frac{9}{8}$ \\
\hline 330 & 2 & $3 A_{1}^{\prime}$ & $4 A_{1}+2 \underline{A_{1}}$ & 0 & 5 & $\frac{9}{5} \frac{3}{6} \frac{3}{5} \frac{3}{4} \frac{0}{5} \frac{0}{4} \frac{0}{7} \frac{0}{6} \| \frac{0}{9} \frac{4}{7} \frac{1}{1} \frac{4}{2} \frac{7}{3} \frac{9}{9}$ \\
\hline 331 & 2 & $3 A_{1}^{\prime}$ & $\underline{A_{1}}+A_{5}$ & 0 & 3 & $\frac{9}{5} \frac{3}{6} \frac{3}{5} \frac{3}{4} \frac{0}{5} \frac{0}{4} \frac{0}{7} \frac{0}{6} \| \frac{0}{9} \frac{4}{7} \frac{1}{1} \frac{1}{7} \frac{2}{4} \frac{4}{2}$ \\
\hline 332 & 2 & $3 A_{1}^{\prime}$ & $E_{6}$ & 0 & 1 & $\frac{9}{5} \frac{3}{6} \frac{3}{5} \frac{3}{4} \frac{0}{5} \frac{0}{4} \frac{0}{7} \frac{0}{6} \| \frac{0}{9} \frac{1}{0} \frac{1}{1} \frac{1}{7} \frac{2}{4} \frac{4}{2}$ \\
\hline 333 & 2 & $3 A_{1}^{\prime}$ & $\underline{A_{1}}+2 A_{3}$ & 0 & 4 & $\frac{9}{5} \frac{3}{6} \frac{3}{5} \frac{3}{4} \frac{0}{5} \frac{0}{4} \frac{0}{7} \frac{0}{6} \| \frac{0}{9} \frac{1}{1} \frac{1}{8} \frac{2}{3} \frac{4}{2} \frac{5}{4} \frac{6}{7}$ \\
\hline 334 & 2 & $3 A_{1}^{\prime}$ & $A_{7}$ & 0 & 2 & $\frac{9}{5} \frac{3}{6} \frac{3}{5} \frac{3}{4} \frac{0}{5} \frac{0}{4} \frac{0}{7} \frac{0}{6} \| \frac{0}{9} \frac{1}{1} \frac{1}{6} \frac{1}{8} \frac{2}{3} \frac{3}{9} \frac{4}{2}$ \\
\hline
\end{tabular}

\begin{tabular}{|c|c|c|c|c|c|c|}
\hline & $\mathrm{d}$ & $D(A)$ & $D(B)$ & $\# E$ & $\# G$ & $\sigma_{A} \| B$ \\
\hline 335 & 2 & $4 A_{1}$ & $A_{0}$ & 0 & 6 & $\frac{9}{5} \frac{3}{5} \frac{3}{6} \frac{3}{4} \frac{0}{5} \frac{0}{4} \frac{0}{7} \frac{0}{6} \|$ \\
\hline 336 & 2 & $4 A_{1}$ & $\underline{A_{1}}$ & 0 & 5 & $\frac{9}{5} \frac{3}{5} \frac{3}{6} \frac{3}{4} \frac{0}{5} \frac{0}{4} \frac{0}{7} \frac{0}{6} \| \frac{4}{7}$ \\
\hline 337 & 2 & $4 A_{1}$ & $2 A_{1}$ & 0 & 4 & $\frac{9}{5} \frac{3}{5} \frac{3}{6} \frac{3}{4} \frac{0}{5} \frac{0}{4} \frac{0}{7} \frac{0}{6} \| \frac{1}{1} \frac{4}{2}$ \\
\hline 338 & 2 & $4 A_{1}$ & $2 \underline{A_{1}}$ & 0 & 4 & $\frac{9}{5} \frac{3}{5} \frac{3}{6} \frac{3}{4} \frac{0}{5} \frac{0}{4} \frac{0}{7} \frac{0}{6} \| \frac{4}{7} \frac{7}{1}$ \\
\hline 339 & 2 & $4 A_{1}$ & $A_{2}$ & 0 & 5 & $\frac{9}{5} \frac{3}{5} \frac{3}{6} \frac{3}{4} \frac{0}{5} \frac{0}{4} \frac{0}{7} \frac{0}{6} \| \frac{1}{2} \frac{4}{4}$ \\
\hline 340 & 2 & $4 A_{1}$ & $2 A_{1}+\underline{A_{1}}$ & 0 & 3 & $\frac{9}{5} \frac{3}{5} \frac{3}{6} \frac{3}{4} \frac{0}{5} \frac{0}{4} \frac{0}{7} \frac{0}{6} \| \frac{4}{7} \frac{1}{1} \frac{4}{2}$ \\
\hline 341 & 2 & $4 A_{1}$ & $3 \underline{A_{1}}$ & 0 & 3 & $\frac{9}{5} \frac{3}{5} \frac{3}{6} \frac{3}{4} \frac{0}{5} \frac{0}{4} \frac{0}{7} \frac{0}{6} \| \frac{4}{7} \frac{7}{1} \frac{0}{a}$ \\
\hline 342 & 2 & $4 A_{1}$ & $\underline{A_{1}}+A_{2}$ & 0 & 4 & $\frac{9}{5} \frac{3}{5} \frac{3}{6} \frac{3}{4} \frac{0}{5} \frac{0}{4} \frac{0}{7} \frac{0}{6} \| \frac{4}{7} \frac{2}{0} \frac{6}{1}$ \\
\hline 343 & 2 & $4 A_{1}$ & $A_{3}$ & 0 & 3 & $\frac{9}{5} \frac{3}{5} \frac{3}{6} \frac{3}{4} \frac{0}{5} \frac{0}{4} \frac{0}{7} \frac{0}{6} \| \frac{4}{7} \frac{1}{7} \frac{2}{4}$ \\
\hline 344 & 2 & $4 A_{1}$ & $2 A_{1}+A_{2}$ & 0 & 3 & $\frac{9}{5} \frac{3}{5} \frac{3}{6} \frac{3}{4} \frac{0}{5} \frac{0}{4} \frac{0}{7} \frac{0}{6} \| 1 \frac{1}{1} \frac{1}{6} \frac{4}{0} \frac{4}{2}$ \\
\hline 345 & 2 & $4 A_{1}$ & $2 \underline{A_{1}}+A_{2}$ & 0 & 3 & $\frac{9}{5} \frac{3}{5} \frac{3}{6} \frac{3}{4} \frac{0}{5} \frac{0}{4} \frac{0}{7} \frac{0}{6} \| \frac{4}{7} \frac{7}{1} \frac{5}{2} \frac{6}{6}$ \\
\hline 346 & 2 & $4 A_{1}$ & $2 A_{2}$ & 0 & 4 & $\frac{9}{5} \frac{3}{5} \frac{3}{6} \frac{3}{4} \frac{0}{5} \frac{0}{4} \frac{0}{7} \frac{0}{6} \| \frac{1}{2} \frac{2}{1} \frac{4}{4} \frac{6}{0}$ \\
\hline 347 & 2 & $4 A_{1}$ & $2 A_{2}$ & 0 & 2 & $\frac{9}{5} \frac{3}{5} \frac{3}{6} \frac{3}{4} \frac{0}{5} \frac{0}{4} \frac{0}{7} \frac{0}{6} \| \frac{1}{1} \frac{1}{7} \frac{2}{4} \frac{4}{2}$ \\
\hline 348 & 2 & $4 A_{1}$ & $4 A_{1}$ & 0 & 3 & $\frac{9}{5} \frac{3}{5} \frac{3}{6} \frac{3}{4} \frac{0}{5} \frac{0}{4} \frac{0}{7} \frac{0}{6} \| \frac{1}{1} \frac{4}{2} \frac{7}{3} \frac{9}{9}$ \\
\hline 349 & 2 & $4 A_{1}$ & $\underline{A_{1}}+A_{3}$ & 0 & 2 & $\frac{9}{5} \frac{3}{5} \frac{3}{6} \frac{3}{4} \frac{0}{5} \frac{0}{4} \frac{0}{7} \frac{0}{6} \| \frac{4}{7} \frac{7}{1} \frac{1}{1} \frac{4}{2}$ \\
\hline 350 & 2 & $4 A_{1}$ & $A_{4}$ & 0 & 3 & $\frac{9}{5} \frac{3}{5} \frac{3}{6} \frac{3}{4} \frac{0}{5} \frac{0}{4} \frac{0}{7} \frac{0}{6} \| \frac{1}{1} \frac{2}{0} \frac{4}{2} \frac{6}{1}$ \\
\hline 351 & 2 & $4 A_{1}$ & $4 A_{1}+\underline{A_{1}}$ & 0 & 2 & $\frac{9}{5} \frac{3}{5} \frac{3}{6} \frac{3}{4} \frac{0}{5} \frac{0}{4} \frac{0}{7} \frac{0}{6} \| \frac{4}{7} \frac{1}{1} \frac{4}{2} \frac{7}{3} \frac{9}{9}$ \\
\hline 352 & 2 & $4 A_{1}$ & $\underline{A_{1}}+2 A_{2}$ & 0 & 3 & $\frac{9}{5} \frac{3}{5} \frac{3}{6} \frac{3}{4} \frac{0}{5} \frac{0}{4} \frac{0}{7} \frac{0}{6} \| \frac{4}{7} \frac{2}{0} \frac{5}{3} \frac{6}{1} \frac{6}{5}$ \\
\hline 353 & 2 & $4 A_{1}$ & $\underline{A_{1}}+A_{4}$ & 0 & 2 & $\frac{9}{5} \frac{3}{5} \frac{3}{6} \frac{3}{4} \frac{0}{5} \frac{0}{4} \frac{0}{7} \frac{0}{6} \| \frac{4}{7} \frac{1}{1} \frac{2}{0} \frac{4}{2} \frac{6}{1}$ \\
\hline 354 & 2 & $4 A_{1}$ & $A_{2}+A_{3}$ & 0 & 2 & $\frac{9}{5} \frac{3}{5} \frac{3}{6} \frac{3}{4} \frac{0}{5} \frac{0}{4} \frac{0}{7} \frac{0}{6} \| \frac{4}{7} \frac{1}{7} \frac{2}{4} \frac{5}{5} \frac{6}{3}$ \\
\hline 355 & 2 & $4 A_{1}$ & $A_{5}$ & 0 & 1 & $\frac{9}{5} \frac{3}{5} \frac{3}{6} \frac{3}{4} \frac{0}{5} \frac{0}{4} \frac{0}{7} \frac{0}{6} \| \frac{4}{7} \frac{1}{1} \frac{1}{7} \frac{2}{4} \frac{4}{2}$ \\
\hline 356 & 2 & $4 A_{1}$ & $2 A_{3}$ & 0 & 1 & $\frac{9}{5} \frac{3}{5} \frac{3}{6} \frac{3}{4} \frac{0}{5} \frac{0}{4} \frac{0}{7} \frac{0}{6} \| \frac{1}{1} \frac{1}{8} \frac{2}{3} \frac{4}{2} \frac{5}{4} \frac{6}{7}$ \\
\hline 357 & 2 & $4 A_{1}$ & $3 \mathrm{~A}_{2}$ & 0 & 3 & $\frac{9}{5} \frac{3}{5} \frac{3}{6} \frac{3}{4} \frac{0}{5} \frac{0}{4} \frac{0}{7} \frac{0}{6} \| 1 \frac{1}{2} \frac{2}{1} \frac{4}{4} \frac{5}{5} \frac{6}{0} \frac{6}{3}$ \\
\hline 358 & 2 & $4 A_{1}$ & $A_{2}+A_{4}$ & 0 & 2 & $\frac{9}{5} \frac{3}{5} \frac{3}{6} \frac{3}{4} \frac{0}{5} \frac{0}{4} \frac{0}{7} \frac{0}{6} \| \frac{1}{1} \frac{1}{6} \frac{2}{1} \frac{4}{0} \frac{4}{2} \frac{6}{0}$ \\
\hline 359 & 2 & $4 A_{1}$ & $A_{6}$ & 0 & 1 & $\frac{9}{5} \frac{3}{5} \frac{3}{6} \frac{3}{4} \frac{0}{5} \frac{0}{4} \frac{0}{7} \frac{0}{6} \| \frac{1}{1} \frac{1}{6} \frac{1}{8} \frac{2}{3} \frac{4}{0} \frac{4}{2}$ \\
\hline 360 & 2 & $D_{4}$ & $A_{0}$ & 8 & 6 & $\frac{3}{f} \frac{9}{6} \frac{3}{4} \frac{3}{5} \frac{3}{8} \frac{4}{1} \frac{0}{6} \frac{0}{7} \|$ \\
\hline 361 & 2 & $D_{4}$ & $\underline{A_{1}}$ & 4 & 5 & $\frac{3}{f} \frac{9}{6} \frac{3}{4} \frac{3}{5} \frac{3}{8} \frac{4}{1} \frac{0}{6} \frac{0}{7} \| \frac{2}{2}$ \\
\hline 362 & 2 & $D_{4}$ & $2 A_{1}$ & 6 & 4 & $\frac{3}{f} \frac{9}{6} \frac{3}{4} \frac{3}{5} \frac{3}{8} \frac{4}{1} \frac{0}{6} \frac{0}{7} \| \frac{2}{3} \frac{6}{5}$ \\
\hline 363 & 2 & $D_{4}$ & $2 \underline{A_{1}}$ & 2 & 4 & $\frac{3}{f} \frac{9}{6} \frac{3}{4} \frac{3}{5} \frac{3}{8} \frac{4}{1} \frac{0}{6} \frac{0}{7} \| \frac{2}{2} \frac{6}{9}$ \\
\hline 364 & 2 & $D_{4}$ & $2 A_{1}+\underline{A_{1}}$ & 3 & 3 & $\frac{3}{f} \frac{9}{6} \frac{3}{4} \frac{3}{5} \frac{3}{8} \frac{4}{1} \frac{0}{6} \frac{0}{7} \| \frac{2}{2} \frac{7}{0} \frac{0}{b}$ \\
\hline 365 & 2 & $D_{4}$ & $3 \underline{A_{1}}$ & 1 & 3 & $\frac{3}{f} \frac{9}{6} \frac{3}{4} \frac{3}{5} \frac{3}{8} \frac{4}{1} \frac{0}{6} \frac{0}{7} \| \frac{2}{2} \frac{6}{9} \frac{0}{c}$ \\
\hline 366 & 2 & $D_{4}$ & $A_{3}$ & 2 & 3 & $\frac{3}{f} \frac{9}{6} \frac{3}{4} \frac{3}{5} \frac{3}{8} \frac{4}{1} \frac{0}{6} \frac{0}{7} \| \frac{1}{7} \frac{5}{5} \frac{2}{2}$ \\
\hline 367 & 2 & $D_{4}$ & $2 A_{2}$ & 4 & 2 & $\frac{3}{f} \frac{9}{6} \frac{3}{4} \frac{3}{5} \frac{3}{8} \frac{4}{1} \frac{0}{6} \frac{0}{7} \| \frac{1}{7} \frac{5}{5} \frac{2}{7} \frac{9}{8}$ \\
\hline 368 & 2 & $D_{4}$ & $4 A_{1}$ & 4 & 3 & $\frac{3}{f} \frac{9}{6} \frac{3}{4} \frac{3}{5} \frac{3}{8} \frac{4}{1} \frac{0}{6} \frac{0}{7} \| \frac{1}{7} \frac{5}{5} \frac{2}{4} \frac{6}{2}$ \\
\hline 369 & 2 & $D_{4}$ & $\underline{A_{1}}+A_{3}$ & 1 & 2 & $\frac{3}{f} \frac{9}{6} \frac{3}{4} \frac{3}{5} \frac{3}{8} \frac{4}{1} \frac{0}{6} \frac{0}{7} \| \frac{1}{7} \frac{5}{5} \frac{2}{2} \frac{0}{c}$ \\
\hline 370 & 2 & $D_{4}$ & $4 A_{1}+\underline{A_{1}}$ & 2 & 2 & $\frac{3}{f} \frac{9}{6} \frac{3}{4} \frac{3}{5} \frac{3}{8} \frac{4}{1} \frac{0}{6} \frac{0}{7} \| \frac{1}{7} \frac{5}{5} \frac{0}{c} \frac{2}{4} \frac{6}{2}$ \\
\hline 371 & 2 & $D_{4}$ & $A_{5}$ & 1 & 1 & $\frac{3}{f} \frac{9}{6} \frac{3}{4} \frac{3}{5} \frac{3}{8} \frac{4}{1} \frac{0}{6} \frac{0}{7} \| \frac{0}{9} \frac{4}{7} \frac{1}{7} \frac{5}{5} \frac{2}{2}$ \\
\hline 372 & 2 & $D_{4}$ & $2 A_{3}$ & 2 & 1 & $\frac{3}{f} \frac{9}{6} \frac{3}{4} \frac{3}{5} \frac{3}{8} \frac{4}{1} \frac{0}{6} \frac{0}{7} \| \frac{0}{9} \frac{4}{7} \frac{1}{5} \frac{5}{4} \frac{6}{2}$ \\
\hline 373 & 2 & $A_{1}+D_{4}$ & $A_{0}$ & 0 & 4 & $\frac{3}{f} \frac{9}{6} \frac{3}{4} \frac{3}{5} \frac{3}{8} \frac{4}{1} \frac{0}{7} \frac{0}{6} \|$ \\
\hline 374 & 2 & $A_{1}+D_{4}$ & $\underline{A_{1}}$ & 0 & 3 & $\frac{3}{f} \frac{9}{6} \frac{3}{4} \frac{3}{5} \frac{3}{8} \frac{4}{1} \frac{0}{7} \frac{0}{6} \| \frac{6}{9}$ \\
\hline 375 & 2 & $A_{1}+D_{4}$ & $2 A_{1}$ & 0 & 2 & $\frac{3}{f} \frac{9}{6} \frac{3}{4} \frac{3}{5} \frac{3}{8} \frac{4}{1} \frac{0}{7} \frac{0}{6} \| \frac{0}{9} \frac{4}{7}$ \\
\hline 376 & 2 & $A_{1}+D_{4}$ & $2 \underline{A_{1}}$ & 0 & 2 & $\frac{3}{f} \frac{9}{6} \frac{3}{4} \frac{3}{5} \frac{3}{8} \frac{4}{1} \frac{0}{7} \frac{0}{6} \| \frac{6}{9} \frac{0}{c}$ \\
\hline 377 & 2 & $A_{1}+D_{4}$ & $A_{2}$ & 0 & 3 & $\frac{3}{f} \frac{9}{6} \frac{3}{4} \frac{3}{5} \frac{3}{8} \frac{4}{1} \frac{0}{7} \frac{0}{6} \| \frac{1}{7} \frac{6}{5}$ \\
\hline 378 & 2 & $A_{1}+D_{4}$ & $\underline{A_{1}}+A_{2}$ & 0 & 2 & $\frac{3}{f} \frac{9}{6} \frac{3}{4} \frac{3}{5} \frac{3}{8} \frac{4}{1} \frac{0}{7} \frac{0}{6} \| \frac{6}{9} \frac{2}{1} \frac{9}{8}$ \\
\hline 379 & 2 & $A_{1}+D_{4}$ & $A_{3}$ & 0 & 1 & $\frac{3}{f} \frac{9}{6} \frac{3}{4} \frac{3}{5} \frac{3}{8} \frac{4}{1} \frac{0}{7} \frac{0}{6} \| \frac{6}{9} \frac{0}{9} \frac{4}{7}$ \\
\hline 380 & 2 & $A_{1}+D_{4}$ & $2 \mathrm{~A}_{2}$ & 0 & 2 & $\frac{3}{f} \frac{9}{6} \frac{3}{4} \frac{3}{5} \frac{3}{8} \frac{4}{1} \frac{0}{7} \frac{0}{6} \| \frac{1}{7} \frac{2}{7} \frac{6}{5} \frac{9}{7}$ \\
\hline 381 & 2 & $A_{1}+D_{4}$ & $4 A_{1}$ & 0 & 1 & $\frac{3}{f} \frac{9}{6} \frac{3}{4} \frac{3}{5} \frac{3}{8} \frac{4}{1} \frac{0}{7} \frac{0}{6} \| \frac{0}{9} \frac{4}{7} \frac{7}{1} \frac{0}{a}$ \\
\hline 382 & 2 & $A_{1}+D_{4}$ & $A_{4}$ & 0 & 1 & $\frac{3}{f} \frac{9}{6} \frac{3}{4} \frac{3}{5} \frac{3}{8} \frac{4}{1} \frac{0}{7} \frac{0}{6} \| \frac{0}{9} \frac{1}{7} \frac{4}{7} \frac{6}{5}$ \\
\hline 383 & 2 & $D_{6}$ & $A_{0}$ & 0 & 2 & $\frac{6}{d} \frac{4}{a} \frac{3}{4} \frac{3}{5} \frac{3}{8} \frac{4}{1} \frac{4}{8} \frac{5}{8}||$ \\
\hline 384 & 2 & $D_{6}$ & $\underline{A_{1}}$ & 0 & 1 & $\frac{6}{d} \frac{4}{a} \frac{3}{4} \frac{3}{5} \frac{3}{8} \frac{4}{1} \frac{4}{8} \frac{5}{8} \| \frac{0}{c}$ \\
\hline 385 & 2 & $D_{6}$ & $A_{2}$ & 0 & 1 & $\frac{6}{d} \frac{4}{a} \frac{3}{4} \frac{3}{5} \frac{3}{8} \frac{4}{1} \frac{4}{8} \frac{5}{8} \| \frac{0}{9} \frac{0}{b}$ \\
\hline 386 & 2 & $E_{7}$ & $A_{0}$ & 0 & 0 & $\frac{8}{a} \frac{4}{a} \frac{4}{b} \frac{4}{c} \frac{4}{d} \frac{4}{e} \frac{4}{f} \frac{5}{a} \|$ \\
\hline
\end{tabular}




\begin{tabular}{|c|c|c|c|c|c|c|c|c|c|c|c|c|c|}
\hline & $\mathrm{d}$ & $D(A)$ & $D(B)$ & $\# E$ & $\# G$ & $\sigma_{A} \| B$ & & $\mathrm{~d}$ & $D(A)$ & $D(B)$ & $\# E$ & $\# G$ & $\sigma_{A} \| B$ \\
\hline 387 & 1 & $A_{0}$ & $A_{0}$ & 240 & 2160 & $\frac{0}{0} \frac{0}{1} \frac{0}{2} \frac{0}{3} \frac{0}{4} \frac{0}{5} \frac{0}{6} \frac{0}{7} \frac{0}{8} \|$ & 443 & 1 & $A_{0}$ & $\underline{A_{3}}+\underline{A_{4}}$ & 12 & 35 & $\frac{0}{0} \frac{0}{1} \frac{0}{2} \frac{0}{3} \frac{0}{4} \frac{0}{5} \frac{0}{6} \frac{0}{7} \frac{0}{8} \| \frac{0}{9} \frac{1}{0} \frac{1}{1} \frac{1}{7} \frac{2}{2} \frac{2}{8} \frac{3}{7}$ \\
\hline 388 & 1 & $A_{0}$ & $\underline{A_{1}}$ & 183 & 1458 & $\frac{0}{0} \frac{0}{1} \frac{0}{2} \frac{0}{3} \frac{0}{4} \frac{0}{5} \frac{0}{6} \frac{0}{7} \frac{0}{8} \| \frac{0}{9}$ & 444 & 1 & $A_{0}$ & $\underline{A_{3}}+\underline{D_{4}}$ & 11 & 32 & $\frac{0}{0} \frac{0}{1} \frac{0}{2} \frac{0}{3} \frac{0}{4} \frac{0}{5} \frac{0}{6} \frac{0}{7} \frac{0}{8} \| \frac{0}{9} \frac{1}{0} \frac{1}{1} \frac{1}{7} \frac{2}{2} \frac{2}{8} \frac{9}{7}$ \\
\hline 389 & 1 & $A_{0}$ & $2 \underline{A_{1}}$ & 138 & 981 & $\frac{0}{0} \frac{0}{1} \frac{0}{2} \frac{0}{3} \frac{0}{4} \frac{0}{5} \frac{0}{6} \frac{0}{7} \frac{0}{8} \| \frac{0}{9} \frac{1}{1}$ & 445 & 1 & $A_{0}$ & $\underline{A_{7}}$ & 8 & 15 & $\frac{0}{0} \frac{0}{1} \frac{0}{2} \frac{0}{3} \frac{0}{4} \frac{0}{5} \frac{0}{6} \frac{0}{7} \frac{0}{8} \| \frac{0}{9} \frac{1}{0} \frac{1}{1} \frac{1}{4} \frac{1}{7} \frac{2}{2} \frac{9}{7}$ \\
\hline 390 & 1 & $A_{0}$ & $\underline{A_{2}}$ & 127 & 828 & $\frac{0}{0} \frac{0}{1} \frac{0}{2} \frac{0}{3} \frac{0}{4} \frac{0}{5} \frac{0}{6} \frac{0}{7} \frac{0}{8} \| \frac{0}{9} \frac{1}{0}$ & 446 & 1 & $A_{0}$ & $\underline{A_{7}}$ & 7 & 15 & $\frac{0}{0} \frac{0}{1} \frac{0}{2} \frac{0}{3} \frac{0}{4} \frac{0}{5} \frac{0}{6} \frac{0}{7} \frac{0}{8} \| \frac{0}{9} \frac{1}{0} \frac{1}{1} \frac{1}{4} \frac{1}{7} \frac{2}{2} \frac{2}{8}$ \\
\hline 391 & 1 & $A_{0}$ & $3 \underline{A_{1}}$ & 103 & 657 & $\frac{0}{0} \frac{0}{1} \frac{0}{2} \frac{0}{3} \frac{0}{4} \frac{0}{5} \frac{0}{7} \frac{0}{8} \| \frac{0}{9} \frac{1}{1} \frac{1}{7}$ & 447 & 1 & $A_{0}$ & $\underline{D_{7}}$ & 5 & 10 & $\frac{0}{0} \frac{0}{1} \frac{0}{2} \frac{0}{3} \frac{0}{4} \frac{0}{5} \frac{0}{6} \frac{0}{7} \frac{0}{8} \| \frac{1}{0} \frac{1}{1} \frac{1}{4} \frac{1}{7} \frac{2}{2} \frac{2}{8} \frac{3}{7}$ \\
\hline 392 & 1 & $A_{0}$ & $\underline{A_{1}}+\underline{A_{2}}$ & 94 & 555 & $\frac{0}{0} \frac{0}{1} \frac{0}{2} \frac{0}{3} \frac{0}{4} \frac{0}{5} \frac{0}{6} \frac{0}{7} \frac{0}{8} \| \frac{0}{9} \frac{1}{0} \frac{1}{4}$ & 448 & 1 & $A_{0}$ & $\underline{E_{7}}$ & 5 & 5 & $\frac{0}{0} \frac{0}{1} \frac{0}{2} \frac{0}{3} \frac{0}{4} \frac{0}{5} \frac{0}{6} \frac{0}{7} \frac{0}{8} \| \frac{0}{9} \frac{1}{0} \frac{1}{1} \frac{1}{4} \frac{1}{7} \frac{2}{2} \frac{3}{7}$ \\
\hline 393 & 1 & $A_{0}$ & $\underline{A_{3}}$ & 83 & 423 & $\frac{0}{0} \frac{0}{1} \frac{0}{2} \frac{0}{3} \frac{0}{4} \frac{0}{5} \frac{0}{6} \frac{0}{7} \frac{0}{8} \| \frac{0}{9} \frac{1}{0} \frac{1}{1}$ & 449 & 1 & $A_{0}$ & $2 \underline{A_{1}}+2 \underline{A_{3}}$ & 11 & 32 & $\frac{0}{0} \frac{0}{1} \frac{0}{2} \frac{0}{3} \frac{0}{4} \frac{0}{5} \frac{0}{6} \frac{0}{7} \frac{0}{8} \| \frac{0}{9} \frac{1}{0} \frac{1}{1} \frac{1}{7} \frac{2}{2} \frac{7}{3} \frac{9}{7} \frac{6}{c}$ \\
\hline 394 & 1 & $A_{0}$ & $2 \underline{A_{1}}+\underline{A_{2}}$ & 69 & 369 & $\frac{0}{0} \frac{0}{1} \frac{0}{2} \frac{0}{3} \frac{0}{4} \frac{0}{5} \frac{0}{6} \frac{0}{7} \frac{0}{8} \| \frac{0}{9} \frac{1}{0} \frac{1}{4} \frac{2}{2}$ & 450 & 1 & $A_{0}$ & $2 \underline{A_{1}}+\underline{D_{6}}$ & 6 & 10 & $\frac{0}{0} \frac{0}{1} \frac{0}{2} \frac{0}{3} \frac{0}{4} \frac{0}{5} \frac{0}{6} \frac{0}{7} \frac{0}{8} \| \frac{0}{9} \frac{1}{1} \frac{1}{4} \frac{1}{7} \frac{2}{8} \frac{5}{9} \frac{6}{4} \frac{9}{7}$ \\
\hline 395 & 1 & $A_{0}$ & $2 \underline{A_{2}}$ & 62 & 313 & $\frac{0}{0} \frac{0}{1} \frac{0}{2} \frac{0}{3} \frac{0}{4} \frac{0}{5} \frac{0}{6} \frac{0}{7} \frac{0}{8} \| \frac{0}{9} \frac{1}{0} \frac{1}{4} \frac{1}{7}$ & 451 & 1 & $A_{0}$ & $2 \underline{A_{4}}$ & 6 & 15 & $\frac{0}{0} \frac{0}{1} \frac{0}{2} \frac{0}{3} \frac{0}{4} \frac{0}{5} \frac{0}{6} \frac{0}{7} \frac{0}{8} \| \frac{0}{9} \frac{1}{0} \frac{1}{1} \frac{1}{4} \frac{2}{2} \frac{2}{8} \frac{9}{4} \frac{9}{7}$ \\
\hline 396 & 1 & $A_{0}$ & $4 \underline{A_{1}}$ & 77 & 438 & $\frac{0}{0} \frac{0}{1} \frac{0}{2} \frac{0}{3} \frac{0}{4} \frac{0}{5} \frac{0}{6} \frac{0}{7} \frac{0}{8} \| \frac{0}{9} \frac{1}{1} \frac{1}{7} \frac{9}{7}$ & 452 & 1 & $A_{0}$ & $2 \underline{D_{4}}$ & 5 & 13 & $\frac{0}{0} \frac{0}{1} \frac{0}{2} \frac{0}{3} \frac{0}{4} \frac{0}{5} \frac{0}{6} \frac{0}{7} \frac{0}{8} \| \frac{0}{9} \frac{1}{0} \frac{1}{1} \frac{1}{7} \frac{2}{2} \frac{2}{8} \frac{2}{f} \frac{9}{7}$ \\
\hline 397 & 1 & $A_{0}$ & $4 \underline{A_{1}}$ & 76 & 438 & $\frac{0}{0} \frac{0}{1} \frac{0}{2} \frac{0}{3} \frac{0}{4} \frac{0}{5} \frac{0}{6} \frac{0}{7} \frac{0}{8} \| \frac{0}{9} \frac{1}{1} \frac{1}{7} \frac{2}{8}$ & $\times$ & 1 & $A_{0}$ & $4 \underline{A_{1}}+\underline{D_{4}}$ & 12 & 32 & $\frac{0}{8} \| \frac{0}{9} \frac{1}{1} \frac{1}{7} \frac{2}{8} \frac{5}{9} \frac{6}{4} \frac{7}{3} \frac{9}{7}$ \\
\hline 398 & 1 & $A_{0}$ & $\underline{A_{1}}+\underline{A_{3}}$ & 60 & 282 & $\frac{0}{0} \frac{0}{1} \frac{0}{2} \frac{0}{3} \frac{0}{4} \frac{0}{5} \frac{0}{6} \frac{0}{7} \frac{0}{8} \| \frac{0}{9} \frac{1}{0} \frac{1}{1} \frac{1}{7}$ & 454 & 1 & $A_{0}$ & $4 \underline{A_{2}}$ & 12 & 38 & $\frac{0}{0} \frac{0}{1} \frac{0}{2} \frac{0}{3} \frac{0}{4} \frac{0}{5} \frac{0}{6} \frac{0}{7} \frac{0}{8} \| \frac{3}{7} \frac{4}{7} \frac{5}{5} \frac{8}{1} \frac{7}{1} \frac{6}{3} \frac{8}{7} \frac{8}{9}$ \\
\hline 399 & 1 & $A_{0}$ & $\underline{A_{4}}$ & 51 & 201 & $\frac{0}{0} \frac{0}{1} \frac{0}{2} \frac{0}{3} \frac{0}{4} \frac{0}{5}$ & $x$ & 1 & $A_{0}$ & $8 \underline{A_{1}}$ & 22 & 85 & $\frac{0}{8} \| \frac{0}{9} \frac{1}{1} \frac{1}{7} \frac{5}{9} \frac{6}{4} \frac{7}{3} \frac{9}{7} \frac{6}{c}$ \\
\hline 400 & 1 & $A_{0}$ & $\underline{D_{4}}$ & 49 & 171 & $\frac{0}{0} \frac{0}{1} \frac{0}{2} \frac{0}{3} \frac{0}{4} \frac{0}{5} \frac{0}{6} \frac{0}{7} \frac{0}{8} \| \frac{1}{0} \frac{1}{1} \frac{1}{4} \frac{3}{7}$ & 456 & 1 & $A_{0}$ & $\underline{A_{1}}+\underline{A_{2}}+\underline{A_{5}}$ & 8 & 19 & $\frac{0}{0} \frac{0}{1} \frac{0}{2} \frac{0}{3} \frac{0}{4} \frac{0}{5} \frac{0}{6} \frac{0}{7} \frac{0}{8} \| \frac{0}{9} \frac{1}{0} \frac{1}{4} \frac{2}{2} \frac{2}{8} \frac{3}{7} \frac{4}{5} \frac{9}{4}$ \\
\hline 401 & 1 & $A_{0}$ & $\overline{2 \underline{A_{1}}}+\underline{A_{3}}$ & 44 & 186 & $\frac{0}{0} \frac{0}{1} \frac{0}{2} \frac{0}{3} \frac{0}{4} \frac{0}{5} \frac{0}{6} \frac{0}{7} \frac{0}{8} \| \frac{0}{9} \frac{1}{0} \frac{1}{1} \frac{1}{7} \frac{9}{7}$ & 457 & 1 & $A_{0}$ & $\underline{A_{1}}+\underline{A_{7}}$ & 5 & 9 & $\frac{0}{0} \frac{0}{1} \frac{0}{2} \frac{0}{3} \frac{0}{4} \frac{0}{5} \frac{0}{6} \frac{0}{7} \frac{0}{8} \| \frac{0}{9} \frac{1}{0} \frac{1}{1} \frac{1}{4} \frac{1}{7} \frac{2}{2} \frac{9}{7} \frac{6}{c}$ \\
\hline 402 & 1 & $A_{0}$ & $2 \underline{A_{1}}+\underline{A_{3}}$ & 43 & 186 & $\frac{0}{0} \frac{0}{1} \frac{0}{2} \frac{0}{3} \frac{0}{4} \frac{0}{5} \frac{0}{6} \frac{0}{7} \frac{0}{8} \| \frac{0}{9} \frac{1}{0} \frac{1}{1} \frac{1}{7} \frac{2}{8}$ & 458 & 1 & $A_{0}$ & $\underline{A_{1}}+\underline{E_{7}}$ & 3 & 3 & $\frac{0}{0} \frac{0}{1} \frac{0}{2} \frac{0}{3} \frac{0}{4} \frac{0}{5} \frac{0}{6} \frac{0}{7} \frac{0}{8} \| \frac{0}{9} \frac{1}{0} \frac{1}{4} \frac{1}{7} \frac{2}{2} \frac{2}{8} \frac{3}{7} \frac{4}{5}$ \\
\hline 403 & 1 & $A_{0}$ & $\overline{3 \overline{A_{1}}}+\overline{\overline{A_{2}}}$ & 50 & 244 & $\frac{0}{8} \| \frac{0}{9} \frac{1}{0} \frac{1}{4} \frac{2}{2} \frac{3}{7}$ & 459 & 1 & $A_{0}$ & $\underline{A_{2}}+\underline{E_{6}}$ & 4 & 6 & $\frac{0}{0} \frac{0}{1} \frac{0}{2} \frac{0}{3} \frac{0}{4} \frac{0}{5} \frac{0}{6} \frac{0}{7} \frac{0}{8} \| \frac{0}{9} \frac{1}{0} \frac{1}{4} \frac{1}{7} \frac{2}{2} \frac{2}{8} \frac{3}{7} \frac{5}{7}$ \\
\hline 404 & 1 & $A_{0}$ & $5 \underline{A_{1}}$ & 56 & 291 & $\frac{0}{0} \frac{0}{1} \frac{0}{2} \frac{0}{3} \frac{0}{4} \frac{0}{5} \frac{0}{6} \frac{0}{7} \frac{0}{8} \| \frac{0}{9} \frac{1}{1} \frac{1}{7} \frac{2}{8} \frac{9}{7}$ & 460 & 1 & $A_{0}$ & $\underline{A_{3}}+\underline{D_{5}}$ & 5 & 11 & $\frac{0}{0} \frac{0}{1} \frac{0}{2} \frac{0}{3} \frac{0}{4} \frac{0}{5} \frac{0}{6} \frac{0}{7} \frac{0}{8} \| \frac{0}{9} \frac{1}{0} \frac{1}{1} \frac{1}{7} \frac{2}{2} \frac{2}{8} \frac{7}{3} \frac{9}{7}$ \\
\hline 405 & 1 & $A_{0}$ & $\underline{A_{1}}+2 \underline{A_{2}}$ & 45 & 205 & $\frac{0}{8} \| \frac{0}{9} \frac{1}{0} \frac{1}{4} \frac{1}{7} \frac{2}{8}$ & 461 & 1 & $A_{0}$ & $\underline{A_{8}}$ & 3 & 5 & $\frac{0}{0} \frac{0}{1} \frac{0}{2} \frac{0}{3} \frac{0}{4} \frac{0}{5} \frac{0}{6} \frac{0}{7} \frac{0}{8} \| \frac{0}{9} \frac{1}{0} \frac{1}{1} \frac{1}{7} \frac{2}{2} \frac{2}{8} \frac{3}{7} \frac{7}{3}$ \\
\hline 406 & 1 & $A_{0}$ & $\underline{A_{1}}+\underline{A_{4}}$ & 36 & 132 & $\frac{0}{0} \frac{0}{1} \frac{0}{2} \frac{0}{3} \frac{0}{4} \frac{0}{5} \frac{0}{6} \frac{0}{7} \frac{0}{8} \| \frac{0}{9} \frac{1}{0} \frac{1}{1} \frac{1}{4} \frac{2}{2}$ & 462 & 1 & $A_{0}$ & $\underline{D_{8}}$ & 2 & 3 & $\frac{0}{0} \frac{0}{1} \frac{0}{2} \frac{0}{3} \frac{0}{4} \frac{0}{5} \frac{0}{6} \frac{0}{7} \frac{0}{8} \| \frac{0}{9} \frac{1}{0} \frac{1}{1} \frac{1}{4} \frac{1}{7} \frac{2}{2} \frac{2}{8} \frac{9}{7}$ \\
\hline 407 & 1 & $A_{0}$ & $\overline{A_{1}}+\overline{D_{4}}$ & 34 & 114 & $\frac{0}{0} \frac{0}{1} \frac{0}{2} \frac{0}{3} \frac{0}{4} \frac{0}{5} \frac{0}{6} \frac{0}{7} \frac{0}{8} \| \frac{1}{0} \frac{1}{1} \frac{1}{4} \frac{2}{2} \frac{3}{7}$ & 463 & 1 & $A_{0}$ & $\underline{E_{8}}$ & 1 & 1 & $\frac{0}{0} \frac{0}{1} \frac{0}{2} \frac{0}{3} \frac{0}{4} \frac{0}{5} \frac{0}{6} \frac{0}{7} \frac{0}{8} \| \frac{0}{9} \frac{1}{0} \frac{1}{1} \frac{1}{4} \frac{1}{7} \frac{2}{2} \frac{2}{8} \frac{3}{7}$ \\
\hline 408 & 1 & $A_{0}$ & $\underline{A_{2}}+\underline{A_{3}}$ & 38 & 158 & $\frac{0}{0} \frac{0}{1} \frac{0}{2} \frac{0}{3} \frac{0}{4} \frac{0}{5} \frac{0}{6} \frac{0}{7} \frac{0}{8} \| \frac{0}{9} \frac{1}{0} \frac{1}{1} \frac{1}{7} \frac{2}{2}$ & 464 & 1 & $A_{1}$ & $A_{0}$ & 126 & 756 & $\frac{0}{0} \frac{0}{2} \frac{0}{1} \frac{0}{3} \frac{0}{4} \frac{0}{5} \frac{0}{6} \frac{0}{7} \frac{0}{8} \|$ \\
\hline 409 & 1 & $A_{0}$ & $\underline{A_{5}}$ & 29 & 91 & $\frac{0}{8} \| \frac{0}{9} \frac{1}{0} \frac{1}{1} \frac{1}{4} \frac{1}{7}$ & 465 & 1 & $A_{1}$ & $\underline{A_{1}}$ & 93 & 504 & $\frac{0}{0} \frac{0}{2} \frac{0}{1} \frac{0}{3} \frac{0}{4} \frac{0}{5}$ \\
\hline 410 & 1 & $A_{0}$ & $\underline{D_{5}}$ & 27 & 66 & $\frac{0}{0} \frac{0}{1} \frac{0}{2} \frac{0}{3} \frac{0}{4} \frac{0}{5} \frac{0}{6} \frac{0}{7} \frac{0}{8} \| \frac{0}{9} \frac{1}{0} \frac{1}{1} \frac{1}{4} \frac{3}{7}$ & 466 & 1 & $A_{1}$ & $2 \underline{A_{1}}$ & 68 & 333 & $\frac{0}{0} \frac{0}{2} \frac{0}{1} \frac{0}{3} \frac{0}{4} \frac{0}{5} \frac{0}{6} \frac{0}{7} \frac{0}{8} \| \frac{2}{8} \frac{9}{1}$ \\
\hline 411 & 1 & $A_{0}$ & $2 \underline{A_{1}}+2 \underline{A_{2}}$ & 32 & 134 & $\frac{0}{0} \frac{0}{1} \frac{0}{2} \frac{0}{3} \frac{0}{4} \frac{0}{5} \frac{0}{6} \frac{0}{7} \frac{0}{8} \| \frac{0}{9} \frac{1}{0} \frac{1}{4} \frac{1}{7} \frac{2}{8} \frac{3}{7}$ & 467 & 1 & $A_{1}$ & $\underline{A_{2}}$ & 61 & 282 & $\frac{0}{0} \frac{0}{2} \frac{0}{1} \frac{0}{3} \frac{0}{4} \frac{0}{5} \frac{0}{6} \frac{0}{7} \frac{0}{8} \| \frac{2}{8} \frac{6}{b}$ \\
\hline 412 & 1 & $A_{0}$ & $2 \underline{A_{1}}+\underline{A_{4}}$ & 25 & 86 & $\frac{0}{0} \frac{0}{1} \frac{0}{2} \frac{0}{3} \frac{0}{4} \frac{0}{5} \frac{0}{6} \frac{0}{7} \frac{0}{8} \| \frac{0}{9} \frac{1}{0} \frac{1}{1} \frac{1}{7} \frac{2}{8} \frac{3}{7}$ & 468 & 1 & $A_{1}$ & $3 \underline{A_{1}}$ & 51 & 219 & $\frac{0}{0} \frac{0}{2} \frac{0}{1} \frac{0}{3} \frac{0}{4} \frac{0}{5} \frac{0}{6} \frac{0}{7} \frac{0}{8} \| \frac{2}{2} \frac{3}{0} \frac{2}{f}$ \\
\hline 413 & 1 & $A_{0}$ & $2 \underline{A_{1}}+\underline{D_{4}}$ & 24 & 75 & $\frac{0}{8} \| \frac{0}{9} \frac{1}{1} \frac{1}{7} \frac{2}{2} \frac{2}{8} \frac{9}{7}$ & 469 & 1 & $A_{1}$ & $3 \underline{A_{1}}$ & 49 & 219 & $\frac{0}{0} \frac{0}{2} \frac{0}{1} \frac{0}{3} \frac{0}{4} \frac{0}{5}$ \\
\hline 414 & 1 & $A_{0}$ & $2 \underline{A_{3}}$ & 23 & 79 & $\frac{0}{0} \frac{0}{1} \frac{0}{2} \frac{0}{3} \frac{0}{4} \frac{0}{5} \frac{0}{6} \frac{0}{7} \frac{0}{8} \| \frac{0}{9} \frac{1}{0} \frac{1}{1} \frac{1}{7} \frac{2}{2} \frac{9}{7}$ & 470 & 1 & $A_{1}$ & $\underline{A_{1}}+\underline{A_{2}}$ & 44 & 183 & $\frac{0}{0} \frac{0}{2} \frac{0}{1} \frac{0}{3} \frac{0}{4} \frac{0}{5} \frac{0}{6} \frac{0}{8} \| \frac{2}{2} \frac{2}{8} \frac{9}{4}$ \\
\hline 415 & 1 & $A_{0}$ & $2 \underline{A_{3}}$ & 22 & 79 & $\frac{0}{8} \| \frac{0}{9} \frac{1}{0} \frac{1}{1} \frac{1}{7} \frac{2}{2} \frac{2}{8}$ & 471 & 1 & $A_{1}$ & $\underline{A_{3}}$ & 37 & 141 & $\frac{0}{0} \frac{0}{2} \frac{0}{1} \frac{0}{3} \frac{0}{4} \frac{0}{5} \frac{0}{7}$ \\
\hline 416 & 1 & $A_{0}$ & $3 \underline{A_{1}}+\underline{A_{3}}$ & 31 & 122 & $\frac{0}{0} \frac{0}{1} \frac{0}{2} \frac{0}{3} \frac{0}{4} \frac{0}{5} \frac{0}{6} \frac{0}{7} \frac{0}{8} \| \frac{0}{9} \frac{1}{0} \frac{1}{1} \frac{1}{7} \frac{2}{8} \frac{9}{7}$ & 472 & 1 & $A_{1}$ & $2 \underline{A_{1}}+\underline{A_{2}}$ & 31 & 119 & $\frac{0}{0} \frac{0}{2} \frac{0}{1} \frac{0}{3} \frac{0}{4} \frac{0}{5} \frac{0}{6} \frac{0}{7} \frac{0}{8} \| \frac{2}{2} \frac{2}{8} \frac{9}{4} \frac{5}{d}$ \\
\hline 417 & 1 & $A_{0}$ & $3 \underline{A_{2}}$ & 29 & 111 & $\frac{0}{0} \frac{0}{1} \frac{0}{2} \frac{0}{3} \frac{0}{4} \frac{0}{5} \frac{0}{6} \frac{0}{7} \frac{0}{8} \| \frac{0}{9} \frac{1}{0} \frac{1}{4} \frac{1}{7} \frac{3}{7} \frac{5}{7}$ & 473 & 1 & $A_{1}$ & $2 \underline{A_{2}}$ & 28 & 97 & $\frac{0}{0} \frac{0}{2} \frac{0}{1} \frac{0}{3} \frac{0}{4} \frac{0}{5}$ \\
\hline 418 & 1 & $A_{0}$ & $4 \underline{A_{1}}+\underline{A_{2}}$ & 36 & 161 & $\frac{0}{0} \frac{0}{1} \frac{0}{2} \frac{0}{3} \frac{0}{4} \frac{0}{5} \frac{0}{6} \frac{0}{7} \frac{0}{8} \| \frac{1}{0} \frac{1}{4} \frac{2}{2} \frac{2}{8} \frac{3}{7} \frac{4}{5}$ & 474 & 1 & $A_{1}$ & $4 \underline{A_{1}}$ & 36 & 144 & $\frac{0}{0} \frac{0}{2} \frac{0}{1} \frac{0}{3} \frac{0}{4} \frac{0}{5} \frac{0}{6} \frac{0}{7} \frac{0}{8} \| \frac{2}{2} \frac{3}{0} \frac{8}{2} \frac{2}{f}$ \\
\hline 419 & 1 & $A_{0}$ & $6 \underline{A_{1}}$ & 41 & 193 & $\frac{0}{0} \frac{0}{1} \frac{0}{2} \frac{0}{3} \frac{0}{4} \frac{0}{5} \frac{0}{6} \frac{0}{7} \frac{0}{8} \| \frac{0}{9} \frac{1}{1} \frac{1}{7} \frac{2}{8} \frac{2}{f} \frac{9}{7}$ & 475 & 1 & $A_{1}$ & $4 \underline{A_{1}}$ & 35 & 144 & $\frac{0}{0} \frac{0}{2} \frac{0}{1} \frac{0}{3} \frac{0}{4} \frac{0}{5}$ \\
\hline 420 & 1 & $A_{0}$ & $\underline{A_{1}}+\underline{A_{2}}+\underline{A_{3}}$ & 27 & 102 & $\frac{0}{0} \frac{0}{1} \frac{0}{2} \frac{0}{3} \frac{0}{4} \frac{0}{5} \frac{0}{6} \frac{0}{7} \frac{0}{8} \| \frac{0}{9} \frac{1}{0} \frac{1}{4} \frac{1}{7} \frac{2}{2} \frac{3}{7}$ & 476 & 1 & $A_{1}$ & $\underline{A_{1}}+\underline{A_{3}}$ & 28 & 90 & $\frac{0}{0} \frac{0}{2} \frac{0}{1} \frac{0}{3} \frac{0}{4} \frac{0}{5} \frac{0}{6}$ \\
\hline 421 & 1 & $A_{0}$ & $\underline{A_{1}}+\underline{A_{5}}$ & 21 & 58 & $\frac{0}{8} \| \frac{0}{9} \frac{1}{0} \frac{1}{1} \frac{1}{4} \frac{1}{7} \frac{9}{7}$ & 477 & 1 & $A_{1}$ & $\underline{A_{1}}+\underline{A_{3}}$ & 26 & 90 & $11 \frac{2}{2} \frac{7}{1} \frac{2}{8} \frac{2}{c}$ \\
\hline 422 & 1 & $A_{0}$ & $\underline{A_{1}}+\underline{A_{5}}$ & 20 & 58 & $\frac{0}{0} \frac{0}{1} \frac{0}{2} \frac{0}{3} \frac{0}{4} \frac{0}{5} \frac{0}{6} \frac{0}{7} \frac{0}{8} \| \frac{0}{9} \frac{1}{0} \frac{1}{1} \frac{1}{4} \frac{1}{7} \frac{2}{8}$ & 478 & 1 & $A_{1}$ & $\underline{A_{4}}$ & 21 & 63 & $\frac{0}{0} \frac{0}{2} \frac{0}{1} \frac{0}{3} \frac{0}{4} \frac{0}{5}$ \\
\hline 423 & 1 & $A_{0}$ & $\underline{A_{1}}+\underline{D_{5}}$ & 18 & 43 & $\frac{0}{0} \frac{0}{1} \frac{0}{2} \frac{0}{3} \frac{0}{4} \frac{0}{5} \frac{0}{6} \frac{0}{7} \frac{0}{8} \| \frac{0}{9} \frac{1}{0} \frac{1}{1} \frac{1}{4} \frac{2}{2} \frac{3}{7}$ & 479 & 1 & $A_{1}$ & $\underline{D_{4}}$ & 19 & 57 & \\
\hline 424 & 1 & $A_{0}$ & $\underline{A_{2}}+\underline{A_{4}}$ & 22 & 72 & $\frac{0}{0} \frac{0}{1} \frac{0}{2} \frac{0}{3} \frac{0}{4} \frac{0}{5} \frac{0}{6} \frac{0}{7} \frac{0}{8} \| \frac{0}{9} \frac{1}{0} \frac{1}{1} \frac{1}{4} \frac{2}{2} \frac{2}{8}$ & 480 & 1 & $A_{1}$ & $2 \underline{A_{1}}+\underline{A_{3}}$ & 19 & 58 & $\frac{0}{0} \frac{0}{2} \frac{0}{1} \frac{0}{3} \frac{0}{4} \frac{0}{5}$ \\
\hline 425 & 1 & $A_{0}$ & $\underline{A_{2}}+\underline{D_{4}}$ & 20 & 64 & $\frac{0}{0} \frac{0}{1} \frac{0}{2} \frac{0}{3} \frac{0}{4} \frac{0}{5} \frac{0}{6} \frac{0}{7} \frac{0}{8} \| \frac{1}{0} \frac{1}{1} \frac{1}{4} \frac{2}{2} \frac{2}{8} \frac{3}{7}$ & 481 & 1 & $A_{1}$ & $\underline{2 A_{1}}+\underline{A_{3}}$ & 18 & 58 & $\| \frac{2}{2} \frac{7}{1} \frac{2}{8} \frac{2}{c} \frac{5}{f}$ \\
\hline 426 & 1 & $A_{0}$ & $\underline{A_{6}}$ & 15 & 39 & $\frac{0}{0} \frac{0}{1} \frac{0}{2} \frac{0}{3} \frac{0}{4} \frac{0}{5} \frac{0}{6} \frac{0}{7} \frac{0}{8} \| \frac{0}{9} \frac{1}{0} \frac{1}{1} \frac{1}{4} \frac{1}{7} \frac{2}{2}$ & 482 & 1 & $A_{1}$ & $3 \underline{A_{1}}+\underline{A_{2}}$ & 22 & 78 & $\frac{0}{0} \frac{0}{2} \frac{0}{1} \frac{0}{3} \frac{0}{4} \frac{0}{5} \frac{0}{6} \frac{0}{7} \frac{0}{8} \| \frac{2}{2} \frac{7}{1} \frac{3}{0} \frac{8}{3} \frac{2}{f}$ \\
\hline 427 & 1 & $A_{0}$ & $\underline{D_{6}}$ & 13 & 26 & $\frac{0}{0} \frac{0}{1} \frac{0}{2} \frac{0}{3} \frac{0}{4} \frac{0}{5} \frac{0}{6} \frac{0}{7} \frac{0}{8} \| \frac{1}{0} \frac{1}{1} \frac{1}{4} \frac{1}{7} \frac{2}{2} \frac{3}{7}$ & 483 & 1 & $A_{1}$ & $5 \underline{A_{1}}$ & 26 & 95 & $\frac{0}{0} \frac{0}{2} \frac{0}{1} \frac{0}{3} \frac{0}{4} \frac{0}{5} \frac{0}{6} \frac{0}{7} \frac{0}{8} \| \frac{2}{2} \frac{3}{0} \frac{8}{2} \frac{2}{f} \frac{5}{e}$ \\
\hline 428 & 1 & $A_{0}$ & $\underline{E_{6}}$ & 13 & 19 & $\frac{0}{0} \frac{0}{1} \frac{0}{2} \frac{0}{3} \frac{0}{4} \frac{0}{5} \frac{0}{6} \frac{0}{7} \frac{0}{8} \| \frac{0}{9} \frac{1}{0} \frac{1}{1} \frac{1}{4} \frac{1}{7} \frac{3}{7}$ & 484 & 1 & $A_{1}$ & $\underline{A_{1}}+2 \underline{A_{2}}$ & 19 & 63 & $\frac{0}{0} \frac{0}{2} \frac{0}{1} \frac{0}{3} \frac{0}{4} \frac{0}{5} \frac{0}{6} \frac{0}{7} \frac{0}{8} \| \frac{2}{2} \frac{7}{1} \frac{3}{0} \frac{8}{3} \frac{0}{f}$ \\
\hline 429 & 1 & $A_{0}$ & $2 \underline{A_{1}}+\underline{A_{2}}+\underline{A_{3}}$ & 19 & 66 & $\frac{0}{0} \frac{0}{1} \frac{0}{2} \frac{0}{3} \frac{0}{4} \frac{0}{5} \frac{0}{6} \frac{0}{7} \frac{0}{8} \| \frac{0}{9} \frac{1}{0} \frac{1}{4} \frac{2}{2} \frac{2}{8} \frac{3}{7} \frac{4}{5}$ & 485 & 1 & $A_{1}$ & $\underline{A_{1}}+\underline{A_{4}}$ & 14 & 40 & $\frac{0}{0} \frac{0}{2} \frac{0}{1} \frac{0}{3} \frac{0}{4} \frac{0}{5} \frac{0}{6} \frac{0}{7} \frac{0}{8} \| \frac{1}{7} \frac{2}{2} \frac{7}{3} \frac{2}{8} \frac{5}{d}$ \\
\hline 430 & 1 & $A_{0}$ & $2 \underline{A_{1}}+\underline{A_{5}}$ & 14 & 37 & $\frac{0}{0} \frac{0}{1} \frac{0}{2} \frac{0}{3} \frac{0}{4} \frac{0}{5} \frac{0}{6} \frac{0}{7} \frac{0}{8} \| \frac{0}{9} \frac{1}{0} \frac{1}{1} \frac{1}{4} \frac{1}{7} \frac{2}{8} \frac{9}{7}$ & 486 & 1 & $A_{1}$ & $\underline{A_{1}}+\underline{D_{4}}$ & 14 & 36 & $\frac{0}{0} \frac{0}{2} \frac{0}{1} \frac{0}{3} \frac{0}{4} \frac{0}{5} \frac{0}{6} \frac{0}{7} \frac{0}{8} \| \frac{1}{7} \frac{5}{6} \frac{2}{2} \frac{2}{8} \frac{2}{f}$ \\
\hline 431 & 1 & $A_{0}$ & $2 \underline{A_{1}}+\underline{D_{5}}$ & 12 & 28 & $\frac{0}{0} \frac{0}{1} \frac{0}{2} \frac{0}{3} \frac{0}{4} \frac{0}{5} \frac{0}{6} \frac{0}{7} \frac{0}{8} \| \frac{1}{0} \frac{1}{4} \frac{1}{7} \frac{2}{2} \frac{2}{8} \frac{3}{7} \frac{4}{5}$ & 487 & 1 & $A_{1}$ & $\underline{A_{2}}+\underline{A_{3}}$ & 16 & 46 & $\frac{0}{0} \frac{0}{2} \frac{0}{1} \frac{0}{3} \frac{0}{4} \frac{0}{5} \frac{0}{6} \frac{0}{7} \frac{0}{8} \| \frac{1}{7} \frac{2}{4} \frac{6}{4} \frac{2}{8} \frac{1}{e}$ \\
\hline 432 & 1 & $A_{0}$ & $\underline{3 A_{1}}+\underline{D_{4}}$ & 17 & 49 & $\frac{0}{0} \frac{0}{1} \frac{0}{2} \frac{0}{3} \frac{0}{4} \frac{0}{5} \frac{0}{6} \frac{0}{7} \frac{0}{8} \| \frac{0}{9} \frac{1}{0} \frac{1}{1} \frac{1}{7} \frac{2}{8} \frac{2}{f} \frac{9}{7}$ & 488 & 1 & $A_{1}$ & $\underline{A_{5}}$ & 13 & 25 & $\frac{0}{0} \frac{0}{2} \frac{0}{1} \frac{0}{3} \frac{0}{4} \frac{0}{5} \frac{0}{6} \frac{0}{7} \frac{0}{8} \| \frac{1}{4} \frac{1}{7} \frac{6}{4} \frac{2}{8} \frac{1}{e}$ \\
\hline 433 & 1 & $A_{0}$ & $4 \underline{A_{1}}+\underline{A_{3}}$ & 22 & 80 & $\frac{0}{0} \frac{0}{1} \frac{0}{2} \frac{0}{3} \frac{0}{4} \frac{0}{5} \frac{0}{6} \frac{0}{7} \frac{0}{8} \| \frac{0}{9} \frac{1}{1} \frac{1}{7} \frac{2}{8} \frac{5}{9} \frac{6}{7}$ & 489 & 1 & $A_{1}$ & $\underline{A_{5}}$ & 11 & 25 & $\frac{0}{0} \frac{0}{2} \frac{0}{1} \frac{0}{3} \frac{0}{4} \frac{0}{5} \frac{0}{6} \frac{0}{7} \frac{0}{8} \| \frac{1}{7} \frac{5}{6} \frac{2}{2} \frac{3}{2} \frac{0}{d}$ \\
\hline$\times$ & 1 & $A_{0}$ & $\underline{7 A_{1}}$ & 30 & 128 & $\frac{0}{0} \frac{0}{1} \frac{0}{2} \frac{0}{3} \frac{0}{4} \frac{0}{5} \frac{0}{6} \frac{0}{7} \frac{0}{8} \| \frac{0}{9} \frac{1}{1} \frac{1}{7} \frac{5}{9} \frac{6}{4} \frac{7}{3} \frac{9}{7}$ & 490 & 1 & $A_{1}$ & $\underline{D_{5}}$ & 9 & 20 & $\frac{0}{0} \frac{0}{2} \frac{0}{1} \frac{0}{3} \frac{0}{4} \frac{0}{5} \frac{0}{6} \frac{0}{7} \frac{0}{8} \| \frac{1}{7} \frac{5}{6} \frac{2}{2} \frac{0}{a} \frac{2}{8}$ \\
\hline 435 & 1 & $A_{0}$ & $\underline{A_{1}}+2 \underline{A_{3}}$ & 16 & 50 & $\frac{0}{0} \frac{0}{1} \frac{0}{2} \frac{0}{3} \frac{0}{4} \frac{0}{5} \frac{0}{6} \frac{0}{7} \frac{0}{8} \| \frac{0}{9} \frac{1}{0} \frac{1}{1} \frac{1}{7} \frac{2}{2} \frac{7}{3} \frac{9}{7}$ & 491 & 1 & $A_{1}$ & $2 \underline{A_{1}}+\underline{D_{4}}$ & 10 & 23 & $\frac{0}{0} \frac{0}{2} \frac{0}{1} \frac{0}{3} \frac{0}{4} \frac{0}{5} \frac{0}{6} \frac{0}{7} \frac{0}{8} \| \frac{1}{7} \frac{5}{6} \frac{2}{2} \frac{2}{8} \frac{2}{e}$ \\
\hline 436 & 1 & $A_{0}$ & $\underline{A_{1}}+3 \underline{A_{2}}$ & 20 & 72 & $\frac{0}{0} \frac{0}{1} \frac{0}{2} \frac{0}{3} \frac{0}{4} \frac{0}{5} \frac{0}{6} \frac{0}{7} \frac{0}{8} \| \frac{0}{9} \frac{1}{0} \frac{1}{4} \frac{1}{7} \frac{2}{8} \frac{3}{7} \frac{5}{7}$ & 492 & 1 & $A_{1}$ & $2 \underline{A_{3}}$ & 9 & 21 & $\frac{0}{0} \frac{0}{2} \frac{0}{1} \frac{0}{3} \frac{0}{4} \frac{0}{5} \frac{0}{6} \frac{0}{7} \frac{0}{8} \| \frac{1}{7} \frac{5}{6} \frac{2}{5} \frac{9}{8} \frac{7}{1}$ \\
\hline 437 & 1 & $A_{0}$ & $\underline{A_{1}}+\underline{A_{2}}+\underline{A_{4}}$ & 15 & 46 & $\frac{0}{0} \frac{0}{1} \frac{0}{2} \frac{0}{3} \frac{0}{4} \frac{0}{5} \frac{0}{6} \frac{0}{7} \frac{0}{8} \| \frac{0}{9} \frac{1}{0} \frac{1}{4} \frac{1}{7} \frac{2}{2} \frac{2}{8} \frac{3}{7}$ & 493 & 1 & $A_{1}$ & $3 \underline{A_{1}}+\underline{A_{3}}$ & 13 & 38 & $\frac{0}{0} \frac{0}{2} \frac{0}{1} \frac{0}{3} \frac{0}{4} \frac{0}{5} \frac{0}{6} \frac{0}{7} \frac{0}{8} \| \frac{1}{7} \frac{2}{4} \frac{0}{a} \frac{2}{8} \frac{9}{2} \frac{5}{d}$ \\
\hline 438 & 1 & $A_{0}$ & $\underline{A_{1}}+\underline{A_{6}}$ & 10 & 24 & $\frac{0}{0} \frac{0}{1} \frac{0}{2} \frac{0}{3} \frac{0}{4} \frac{0}{5} \frac{0}{6} \frac{0}{7} \frac{0}{8} \| \frac{0}{9} \frac{1}{1} \frac{1}{4} \frac{1}{7} \frac{2}{2} \frac{2}{8} \frac{3}{7}$ & 494 & 1 & $A_{1}$ & $3 \underline{A_{2}}$ & 11 & 33 & $\frac{0}{0} \frac{0}{2} \frac{0}{1} \frac{0}{3} \frac{0}{4} \frac{0}{5} \frac{0}{6} \frac{0}{7} \frac{0}{8} \| \frac{2}{2} \frac{7}{1} \frac{0}{a} \frac{3}{0} \frac{8}{3} \frac{0}{f}$ \\
\hline 439 & 1 & $A_{0}$ & $\underline{A_{1}}+\underline{D_{6}}$ & 9 & 16 & $\frac{0}{0} \frac{0}{1} \frac{0}{2} \frac{0}{3} \frac{0}{4} \frac{0}{5} \frac{0}{6} \frac{0}{7} \frac{0}{8} \| \frac{0}{9} \frac{1}{1} \frac{1}{4} \frac{1}{7} \frac{2}{2} \frac{2}{8} \frac{9}{7}$ & 495 & 1 & $A_{1}$ & $6 \underline{A_{1}}$ & 19 & 63 & $\frac{0}{0} \frac{0}{2} \frac{0}{1} \frac{0}{3} \frac{0}{4} \frac{0}{5} \frac{0}{6} \frac{0}{7} \frac{0}{8} \| \frac{2}{2} \frac{7}{1} \frac{3}{0} \frac{8}{2} \frac{2}{f} \frac{5}{e}$ \\
\hline 440 & 1 & $A_{0}$ & $\underline{A_{1}}+\underline{E_{6}}$ & 8 & 12 & $\frac{0}{0} \frac{0}{1} \frac{0}{2} \frac{0}{3} \frac{0}{4} \frac{0}{5} \frac{0}{6} \frac{0}{7} \frac{0}{8} \| \frac{0}{9} \frac{1}{0} \frac{1}{1} \frac{1}{4} \frac{1}{7} \frac{2}{8} \frac{3}{7}$ & 496 & 1 & $A_{1}$ & $\underline{A_{1}}+\underline{A_{2}}+\underline{A_{3}}$ & 11 & 30 & $\frac{0}{0} \frac{0}{2} \frac{0}{1} \frac{0}{3} \frac{0}{4} \frac{0}{5} \frac{0}{6} \frac{0}{7} \frac{0}{8} \| \frac{1}{7} \frac{2}{2} \frac{7}{3} \frac{3}{1} \frac{7}{9} \frac{1}{a}$ \\
\hline 441 & 1 & $A_{0}$ & $\underline{A_{2}}+\underline{A_{5}}$ & 12 & 30 & $\frac{0}{0} \frac{0}{1} \frac{0}{2} \frac{0}{3} \frac{0}{4} \frac{0}{5} \frac{0}{6} \frac{0}{7} \frac{0}{8} \| \frac{0}{9} \frac{1}{0} \frac{1}{4} \frac{1}{7} \frac{2}{2} \frac{3}{7} \frac{5}{7}$ & 497 & 1 & $A_{1}$ & $\underline{A_{1}}+\underline{A_{5}}$ & 8 & 16 & $\frac{0}{0} \frac{0}{2} \frac{0}{1} \frac{0}{3} \frac{0}{4} \frac{0}{5} \frac{0}{6} \frac{0}{7} \frac{0}{8} \| \frac{1}{4} \frac{1}{7} \frac{2}{2} \frac{0}{a} \frac{2}{8} \frac{3}{e}$ \\
\hline 442 & 1 & $A_{0}$ & $\underline{A_{2}}+\underline{D_{5}}$ & 10 & 23 & $\frac{0}{0} \frac{0}{1} \frac{0}{2} \frac{0}{3} \frac{0}{4} \frac{0}{5} \frac{0}{6} \frac{0}{7} \frac{0}{8} \| \frac{0}{9} \frac{1}{0} \frac{1}{1} \frac{1}{4} \frac{2}{2} \frac{2}{8} \frac{3}{7}$ & 498 & 1 & $A_{1}$ & $\underline{A_{1}}+\underline{A_{5}}$ & 7 & 16 & $\frac{0}{0} \frac{0}{2} \frac{0}{1} \frac{0}{3} \frac{0}{4} \frac{0}{5} \frac{0}{6} \frac{0}{7} \frac{0}{8} \| \frac{1}{7} \frac{5}{6} \frac{2}{5} \frac{9}{8} \frac{2}{8} \frac{9}{1}$ \\
\hline
\end{tabular}


d $D(A) \quad D(B)$

\#E \#G $\sigma_{A} \| B$

d $D(A) D(B)$

\#E $\quad \# G \quad \sigma_{A} \| B$

\begin{tabular}{|c|c|c|c|c|c|c|c|c|c|c|c|c|c|}
\hline 499 & 1 & $A_{1}$ & $\underline{A_{1}}+\underline{D_{5}}$ & 6 & 13 & $\frac{0}{0} \frac{0}{2} \frac{0}{1} \frac{0}{3} \frac{0}{4} \frac{0}{5} \frac{0}{6} \frac{0}{7} \frac{0}{8} \| \frac{1}{4} \frac{1}{7} \frac{5}{7} \frac{2}{2} \frac{2}{8} \frac{5}{d}$ & 555 & 1 & $2 A_{1}$ & $\underline{D_{5}}$ & 3 & 6 & $\frac{0}{0} \frac{0}{2} \frac{0}{1} \frac{0}{4} \frac{0}{3} \frac{0}{5} \frac{0}{6} \frac{0}{7} \frac{0}{8} \| \frac{1}{7} \frac{9}{7} \frac{2}{2} \frac{7}{3} \frac{2}{8}$ \\
\hline 500 & 1 & $A_{1}$ & $\underline{A_{2}}+\underline{A_{4}}$ & 8 & 20 & $\frac{0}{0} \frac{0}{2} \frac{0}{1} \frac{0}{3} \frac{0}{4} \frac{0}{5} \frac{0}{6} \frac{0}{7} \frac{0}{8} \| \frac{1}{7} \frac{5}{6} \frac{2}{4} \frac{6}{8} \frac{2}{8} \frac{1}{c}$ & 556 & 1 & $2 A_{1}$ & $2 A_{1}+\underline{A_{4}}$ & 5 & 12 & $\frac{0}{0} \frac{0}{2} \frac{0}{1} \frac{0}{4} \frac{0}{3} \frac{0}{5} \frac{0}{6} \frac{0}{7} \frac{0}{8} \| \frac{4}{2} \frac{1}{7} \frac{2}{2} \frac{2}{8} \frac{3}{a} \frac{3}{d}$ \\
\hline 501 & 1 & $A_{1}$ & $\overline{A_{6}}$ & 5 & 9 & $\frac{0}{0} \frac{0}{2} \frac{0}{1} \frac{0}{3} \frac{0}{4} \frac{0}{5} \frac{0}{6} \frac{0}{7} \frac{0}{8} \| \frac{1}{4} \frac{1}{7} \frac{5}{7} \frac{2}{2} \frac{7}{4} \frac{7}{9}$ & 557 & 1 & $2 A_{1}$ & $2 A_{1}+D_{4}$ & 14 & 23 & $\frac{0}{0} \frac{0}{2} \frac{0}{1} \frac{0}{4} \frac{0}{3} \frac{0}{5} \frac{0}{6} \frac{0}{7} \frac{0}{8} \| \frac{5}{0} \frac{5}{3} \frac{2}{2} \frac{3}{b} \frac{3}{c}$ \\
\hline 502 & 1 & $A_{1}$ & $\underline{D_{6}}$ & 5 & 6 & $\frac{0}{0} \frac{0}{2} \frac{0}{1} \frac{0}{3} \frac{0}{4} \frac{0}{5} \frac{0}{6} \frac{0}{7} \frac{0}{8} \| \frac{1}{4} \frac{4}{7} \frac{1}{7} \frac{2}{2} \frac{0}{a} \frac{2}{8}$ & 558 & 1 & $2 A_{1}$ & $2 \underline{A_{1}}+D_{4}$ & 8 & 15 & $\frac{0}{0} \frac{0}{2} \frac{0}{1} \frac{0}{4} \frac{0}{3} \frac{0}{5} \frac{0}{6} \frac{0}{7} \frac{0}{8} \| \frac{5}{0} \frac{5}{3} \frac{2}{2} \frac{2}{8} \frac{5}{f}$ \\
\hline 503 & 1 & $A_{1}$ & $\underline{E_{6}}$ & 3 & 5 & $\frac{0}{0} \frac{0}{2} \frac{0}{1} \frac{0}{3} \frac{0}{4} \frac{0}{5} \frac{0}{6} \frac{0}{7} \frac{0}{8} \| \frac{1}{1} \frac{4}{2} \frac{1}{7} \frac{5}{6} \frac{2}{2} \frac{2}{8}$ & 559 & 1 & $2 A_{1}$ & $2 A_{1}+\underline{D_{4}}$ & 6 & 11 & $\frac{0}{0} \frac{0}{2} \frac{0}{1} \frac{0}{4} \frac{0}{3} \frac{0}{5} \frac{0}{6} \frac{0}{7} \frac{0}{8} \| \frac{1}{7} \frac{9}{7} \frac{2}{2} \frac{2}{8} \frac{3}{a}$ \\
\hline 504 & 1 & $A_{1}$ & $3 \underline{A_{1}}+\underline{D_{4}}$ & 7 & 15 & $\frac{0}{0} \frac{0}{2} \frac{0}{1} \frac{0}{3} \frac{0}{4} \frac{0}{5} \frac{0}{6} \frac{0}{7} \frac{0}{8} \| \frac{1}{4} \frac{1}{7} \frac{2}{2} \frac{0}{a} \frac{3}{2} \frac{0}{d} \frac{3}{e}$ & 560 & 1 & $2 A_{1}$ & $2 \underline{A_{1}}+\underline{D_{4}}$ & 4 & 7 & $\frac{0}{0} \frac{0}{2} \frac{0}{1} \frac{0}{4} \frac{0}{3} \frac{0}{5} \frac{0}{6} \frac{0}{7} \frac{0}{8} \| \frac{1}{7} \frac{9}{7} \frac{2}{2} \frac{2}{8} \frac{2}{f} \frac{3}{e}$ \\
\hline$\times$ & 1 & $A_{1}$ & $7 \underline{A_{1}}$ & 14 & 42 & $\frac{0}{0} \frac{0}{2} \frac{0}{1} \frac{0}{3} \frac{0}{4} \frac{0}{5} \frac{0}{6} \frac{0}{7} \frac{0}{8} \| \frac{1}{7} \frac{2}{4} \frac{0}{a} \frac{3}{2} \frac{0}{f} \frac{1}{c} \frac{3}{e}$ & 561 & 1 & $2 A_{1}$ & $2 A_{3}$ & 15 & 27 & $\frac{0}{0} \frac{0}{2} \frac{0}{1} \frac{0}{4} \frac{0}{3} \frac{0}{5} \frac{0}{6} \frac{0}{7} \frac{0}{8} \| \frac{5}{0} \frac{5}{3} \frac{6}{1} \frac{6}{2} \frac{2}{8} \frac{9}{3}$ \\
\hline 506 & 1 & $A_{1}$ & $\underline{A_{1}}+2 \underline{A_{3}}$ & 6 & 14 & $\frac{0}{0} \frac{0}{2} \frac{0}{1} \frac{0}{3} \frac{0}{4} \frac{0}{5} \frac{0}{6} \frac{0}{7} \frac{0}{8} \| \frac{1}{7} \frac{5}{6} \frac{2}{5} \frac{9}{8} \frac{3}{1} \frac{7}{9} \frac{1}{a}$ & 562 & 1 & $2 A_{1}$ & $\underline{A_{3}}+A_{3}$ & 7 & 15 & $\frac{0}{0} \frac{0}{2} \frac{0}{1} \frac{0}{4} \frac{0}{3} \frac{0}{5} \frac{0}{6} \frac{0}{7} \frac{0}{8} \| \frac{5}{0} \frac{5}{3} \frac{2}{3} \frac{9}{8} \frac{2}{8} \frac{9}{3}$ \\
\hline 507 & 1 & $A_{1}$ & $\underline{A_{1}}+\underline{D_{6}}$ & 3 & 4 & $\frac{0}{0} \frac{0}{2} \frac{0}{1} \frac{0}{3} \frac{0}{4} \frac{0}{5} \frac{0}{6} \frac{0}{7} \frac{0}{8} \| \frac{1}{1} \frac{1}{4} \frac{1}{7} \frac{9}{7} \frac{2}{2} \frac{2}{8} \frac{3}{e}$ & 563 & 1 & $2 A_{1}$ & $A_{3}+\underline{A_{3}}$ & 6 & 15 & $\frac{0}{0} \frac{0}{2} \frac{0}{1} \frac{0}{4} \frac{0}{3} \frac{0}{5} \frac{0}{6} \frac{0}{7} \frac{0}{8} \| \frac{4}{2} \frac{1}{7} \frac{6}{0} \frac{6}{3} \frac{2}{8} \frac{9}{3}$ \\
\hline 508 & 1 & $A_{1}$ & $\underline{A_{2}}+\underline{A_{5}}$ & 4 & 8 & $\frac{0}{0} \frac{0}{2} \frac{0}{1} \frac{0}{3} \frac{0}{4} \frac{0}{5} \frac{0}{6} \frac{0}{7} \frac{0}{8} \| \frac{1}{4} \frac{1}{9} \frac{9}{7} \frac{2}{2} \frac{7}{1} \frac{3}{0} \frac{8}{3}$ & 564 & 1 & $2 A_{1}$ & $2 \underline{A_{3}}$ & 3 & 7 & $\frac{0}{0} \frac{0}{2} \frac{0}{1} \frac{0}{4} \frac{0}{3} \frac{0}{5} \frac{0}{6} \frac{0}{7} \frac{0}{8} \| \frac{1}{7} \frac{9}{7} \frac{5}{9} \frac{6}{4} \frac{2}{8} \frac{9}{3}$ \\
\hline 509 & 1 & $A_{1}$ & $\underline{A_{7}}$ & 2 & 3 & $\frac{0}{0} \frac{0}{2} \frac{0}{1} \frac{0}{3} \frac{0}{4} \frac{0}{5} \frac{0}{6} \frac{0}{7} \frac{0}{8} \| \frac{1}{1} \frac{4}{2} \frac{1}{7} \frac{5}{6} \frac{2}{2} \frac{3}{1} \frac{7}{9}$ & 565 & 1 & $2 A_{1}$ & $2 A_{1}+\underline{A_{1}}+A_{3}$ & 17 & 36 & $\frac{0}{0} \frac{0}{2} \frac{0}{1} \frac{0}{4} \frac{0}{3} \frac{0}{5} \frac{0}{6} \frac{0}{7} \frac{0}{8} \| \frac{1}{7} \frac{6}{0} \frac{6}{3} \frac{2}{8} \frac{3}{b} \frac{3}{c}$ \\
\hline 510 & 1 & $A_{1}$ & $\overline{E_{7}}$ & 1 & 1 & $\frac{0}{0} \frac{0}{2} \frac{0}{1} \frac{0}{3} \frac{0}{4} \frac{0}{5} \frac{0}{6} \frac{0}{7} \frac{0}{8} \| \frac{3}{7} \frac{1}{1} \frac{1}{4} \frac{4}{7} \frac{1}{7} \frac{2}{2} \frac{2}{8}$ & 566 & 1 & $2 A_{1}$ & $3 \underline{A_{1}}+\overline{A_{3}}$ & 11 & 24 & $\frac{0}{0} \frac{0}{2} \frac{0}{1} \frac{0}{4} \frac{0}{3} \frac{0}{5} \frac{0}{6} \frac{0}{7} \frac{0}{8} \| \frac{1}{7} \frac{6}{0} \frac{6}{3} \frac{2}{8} \frac{2}{f} \frac{3}{e}$ \\
\hline 511 & 1 & $2 A_{1}$ & $A_{0}$ & 60 & 252 & $\frac{0}{0} \frac{0}{2} \frac{0}{1} \frac{0}{4} \frac{0}{3} \frac{0}{5} \frac{0}{6} \frac{0}{7} \frac{0}{8} \|$ & 567 & 1 & $2 A_{1}$ & $2 A_{1}+\underline{A_{1}}+\underline{A_{3}}$ & 9 & 18 & $\frac{0}{0} \frac{0}{2} \frac{0}{1} \frac{0}{4} \frac{0}{3} \frac{0}{5} \frac{0}{6} \frac{0}{7} \frac{0}{8} \| \frac{1}{7} \frac{5}{9} \frac{6}{4} \frac{2}{8} \frac{3}{a} \frac{3}{d}$ \\
\hline 512 & 1 & $2 A_{1}$ & $\underline{A_{1}}$ & 43 & 162 & $\frac{0}{0} \frac{0}{2} \frac{0}{1} \frac{0}{4} \frac{0}{3} \frac{0}{5} \frac{0}{6} \frac{0}{7} \frac{0}{8} \| \frac{2}{8}$ & 568 & 1 & $2 A_{1}$ & $4 A_{1}+\underline{A_{2}}$ & 16 & 41 & $\frac{0}{0} \frac{0}{2} \frac{0}{1} \frac{0}{4} \frac{0}{3} \frac{0}{5} \frac{0}{6} \frac{0}{7} \frac{0}{8} \| \frac{1}{7} \frac{2}{2} \frac{7}{5} \frac{7}{8} \frac{3}{b} \frac{3}{c}$ \\
\hline 513 & 1 & $2 A_{1}$ & $2 A_{1}$ & 50 & 171 & $\frac{0}{0} \frac{0}{2} \frac{0}{1} \frac{0}{4} \frac{0}{3} \frac{0}{5} \frac{0}{6} \frac{0}{7} \frac{0}{8} \| \frac{7}{5} \frac{7}{8}$ & 569 & 1 & $2 A_{1}$ & $2 A_{1}+2 \underline{A_{1}}+\underline{A_{2}}$ & 10 & 25 & $\frac{0}{0} \frac{0}{2} \frac{0}{1} \frac{0}{4} \frac{0}{3} \frac{0}{5} \frac{0}{6} \frac{0}{7} \frac{0}{8} \| \frac{1}{7} \frac{2}{2} \frac{7}{4} \frac{7}{9} \frac{3}{a} \frac{3}{d}$ \\
\hline 514 & 1 & $2 A_{1}$ & $2 \underline{A_{1}}$ & 34 & 105 & $\frac{0}{0} \frac{0}{2} \frac{0}{1} \frac{0}{4} \frac{0}{3} \frac{0}{5} \frac{0}{6} \frac{0}{7} \frac{0}{8} \| \frac{2}{8} \frac{1}{c}$ & 570 & 1 & $2 A_{1}$ & $4 A_{1}+2 \underline{A_{1}}$ & 21 & 49 & $\frac{0}{0} \frac{0}{2} \frac{0}{1} \frac{0}{4} \frac{0}{3} \frac{0}{5} \frac{0}{6} \frac{0}{7} \frac{0}{8} \| \frac{6}{0} \frac{6}{3} \frac{2}{9} \frac{0}{e} \frac{3}{b} \frac{3}{c}$ \\
\hline 515 & 1 & $2 A_{1}$ & $2 \underline{A_{1}}$ & 30 & 105 & $\frac{0}{0} \frac{0}{2} \frac{0}{1} \frac{0}{4} \frac{0}{3} \frac{0}{5} \frac{0}{6} \frac{0}{7} \| \frac{2}{8} \frac{9}{3}$ & 571 & 1 & $2 A_{1}$ & $4 A_{1}+2 \underline{A_{1}}$ & 19 & 49 & $\frac{0}{0} \frac{0}{2} \frac{0}{1} \frac{0}{4} \frac{0}{3} \frac{0}{5} \frac{0}{6} \frac{0}{7} \frac{0}{8} \| \frac{1}{7} \frac{5}{9} \frac{7}{5} \frac{7}{8} \frac{3}{b} \frac{3}{c}$ \\
\hline 516 & 1 & $2 A_{1}$ & $\underline{A_{2}}$ & 27 & 84 & $\frac{0}{0} \frac{0}{2} \frac{0}{1} \frac{0}{4} \frac{0}{3} \frac{0}{5} \frac{0}{6} \frac{0}{7} \frac{0}{8} \| \frac{2}{8} \frac{6}{b}$ & 572 & 1 & $2 A_{1}$ & $4 \underline{A_{1}}+2 A_{1}$ & 13 & 31 & $\frac{0}{0} \frac{0}{2} \frac{0}{1} \frac{0}{4} \frac{0}{3} \frac{0}{5} \frac{0}{6} \frac{0}{7} \frac{0}{8} \| \frac{6}{0} \frac{6}{3} \frac{2}{9} \frac{9}{4} \frac{5}{f}$ \\
\hline 517 & 1 & $2 A_{1}$ & $2 A_{1}+\underline{A_{1}}$ & 35 & 111 & $\frac{0}{0} \frac{0}{2} \frac{0}{1} \frac{0}{4} \frac{0}{3} \frac{0}{5} \frac{0}{6} \frac{0}{7} \frac{0}{8} \| \frac{2}{8} \frac{3}{a} \frac{3}{d}$ & 573 & 1 & $2 A_{1}$ & $6 \underline{A_{1}}$ & 9 & 21 & $\frac{0}{0} \frac{0}{2} \frac{0}{1} \frac{0}{4} \frac{0}{3} \frac{0}{5} \frac{0}{6} \frac{0}{7} \frac{0}{8} \| \frac{2}{2} \frac{9}{9} \frac{3}{0} \frac{2}{f} \frac{3}{e}$ \\
\hline 518 & 1 & $2 A_{1}$ & $3 \underline{A_{1}}$ & 23 & 69 & $\frac{0}{0} \frac{0}{2} \frac{0}{1} \frac{0}{4} \frac{0}{3} \frac{0}{5} \frac{0}{6} \frac{0}{8} \| \frac{2}{8} \frac{9}{3} \frac{1}{c}$ & 574 & 1 & $2 A_{1}$ & $\underline{A_{1}}+D_{5}$ & 6 & 9 & $\frac{0}{0} \frac{0}{2} \frac{0}{1} \frac{0}{4} \frac{0}{3} \frac{0}{5} \frac{0}{6} \frac{0}{7} \frac{0}{8} \| \frac{4}{3} \frac{4}{6} \frac{1}{7} \frac{2}{8} \frac{2}{f}$ \\
\hline 519 & 1 & $2 A_{1}$ & $3 \underline{A_{1}}$ & 21 & 69 & $\frac{0}{0} \frac{0}{2} \frac{0}{1} \frac{0}{4} \frac{0}{3} \frac{0}{5} \frac{0}{6} \frac{0}{7} \frac{0}{8} \| \frac{2}{2} \frac{3}{0} \frac{2}{f}$ & 575 & 1 & $2 A_{1}$ & $\underline{A_{2}}+D_{4}$ & 6 & 12 & $\frac{0}{0} \frac{0}{2} \frac{0}{1} \frac{0}{4} \frac{0}{3} \frac{0}{5} \frac{0}{6} \frac{0}{7} \frac{0}{8} \| \frac{4}{2} \frac{5}{0} \frac{5}{3} \frac{2}{2} \frac{9}{4}$ \\
\hline 520 & 1 & $2 A_{1}$ & $\underline{A_{1}}+\underline{A_{2}}$ & 18 & 55 & $\frac{0}{0} \frac{0}{2} \frac{0}{1} \frac{0}{4} \frac{0}{3} \frac{0}{5} \frac{0}{6} \frac{0}{7} \frac{0}{8} \| \frac{2}{2} \frac{2}{8} \frac{9}{4}$ & 576 & 1 & $2 A_{1}$ & $D_{6}$ & 3 & 6 & $\frac{0}{0} \frac{0}{2} \frac{0}{1} \frac{0}{4} \frac{0}{3} \frac{0}{5} \frac{0}{6} \frac{0}{7} \frac{0}{8} \| \frac{4}{3} \frac{4}{6} \frac{1}{7} \frac{2}{2} \frac{7}{3} \frac{2}{8}$ \\
\hline 521 & 1 & $2 A_{1}$ & $A_{3}$ & 33 & 81 & $\frac{0}{0} \frac{0}{2} \frac{0}{1} \frac{0}{4} \frac{0}{3} \frac{0}{5} \frac{0}{6} \frac{0}{7} \frac{0}{8} \| \frac{7}{3} \frac{7}{5} \frac{7}{8}$ & 577 & 1 & $2 A_{1}$ & $\underline{D_{6}}$ & 1 & 2 & $\frac{0}{0} \frac{0}{2} \frac{0}{1} \frac{0}{4} \frac{0}{3} \frac{0}{5} \frac{0}{6} \frac{0}{7} \frac{0}{8} \| \frac{4}{2} \frac{4}{7} \frac{1}{7} \frac{2}{2} \frac{7}{3} \frac{2}{8}$ \\
\hline 522 & 1 & $2 A_{1}$ & $\underline{A_{3}}$ & 19 & 39 & $\frac{0}{0} \frac{0}{2} \frac{0}{1} \frac{0}{4} \frac{0}{3} \frac{0}{5} \frac{0}{6} \frac{0}{7} \frac{0}{8} \| \frac{7}{3} \frac{2}{8} \frac{1}{c}$ & 578 & 1 & $2 A_{1}$ & $2 A_{1}+\underline{A_{2}}+A_{3}$ & 9 & 20 & $\frac{0}{0} \frac{0}{2} \frac{0}{1} \frac{0}{4} \frac{0}{3} \frac{0}{5} \frac{0}{6} \frac{0}{7} \frac{0}{8} \| \frac{4}{2} \frac{1}{7} \frac{6}{0} \frac{6}{3} \frac{2}{8} \frac{3}{b} \frac{3}{c}$ \\
\hline 523 & 1 & $2 A_{1}$ & $\underline{A_{3}}$ & 15 & 39 & $\frac{0}{0} \frac{0}{2} \frac{0}{1} \frac{0}{4} \frac{0}{3} \frac{0}{5} \frac{0}{6} \frac{0}{7} \frac{0}{8} \| \frac{2}{2} \frac{2}{8} \frac{6}{a}$ & 579 & 1 & $2 A_{1}$ & $2 A_{1}+D_{5}$ & 6 & 10 & $\frac{0}{0} \frac{0}{2} \frac{0}{1} \frac{0}{4} \frac{0}{3} \frac{0}{5} \frac{0}{6} \frac{0}{7} \frac{0}{8} \| \frac{4}{3} \frac{4}{6} \frac{1}{7} \frac{2}{2} \frac{2}{8} \frac{3}{b} \frac{3}{c}$ \\
\hline 524 & 1 & $2 A_{1}$ & $2 A_{1}+\underline{A_{2}}$ & 21 & 59 & $\frac{0}{0} \frac{0}{2} \frac{0}{1} \frac{0}{4} \frac{0}{3} \frac{0}{5} \frac{0}{6} \frac{0}{7} \frac{0}{8} \| \frac{2}{2} \frac{2}{8} \frac{3}{a} \frac{3}{d}$ & 580 & 1 & $2 A_{1}$ & $2 \underline{A_{1}}+D_{5}$ & 4 & 6 & $\frac{0}{0} \frac{0}{2} \frac{0}{1} \frac{0}{4} \frac{0}{3} \frac{0}{5} \frac{0}{6} \frac{0}{7} \frac{0}{8} \| \frac{4}{3} \frac{4}{6} \frac{1}{7} \frac{2}{2} \frac{2}{8} \frac{2}{f} \frac{3}{e}$ \\
\hline 525 & 1 & $2 A_{1}$ & $2 \underline{A_{1}}+\underline{A_{2}}$ & 13 & 37 & $\frac{0}{0} \frac{0}{2} \frac{0}{1} \frac{0}{4} \frac{0}{3} \frac{0}{5} \frac{0}{6} \frac{0}{7} \frac{0}{8} \| \frac{2}{2} \frac{2}{8} \frac{9}{4} \frac{5}{f}$ & 581 & 1 & $2 A_{1}$ & $2 A_{1}+\underline{D_{5}}$ & 2 & 4 & $\frac{0}{0} \frac{0}{2} \frac{0}{1} \frac{0}{4} \frac{0}{3} \frac{0}{5} \frac{0}{6} \frac{0}{7} \frac{0}{8} \| \frac{4}{2} \frac{4}{7} \frac{1}{7} \frac{2}{2} \frac{2}{8} \frac{3}{a} \frac{3}{d}$ \\
\hline 526 & 1 & $2 A_{1}$ & $2 \underline{A_{2}}$ & 10 & 29 & $\frac{0}{0} \frac{0}{2} \frac{0}{1} \frac{0}{4} \frac{0}{3} \frac{0}{5} \frac{0}{6} \frac{0}{7} \frac{0}{8} \| \frac{1}{7} \frac{5}{9} \frac{2}{8} \frac{9}{3}$ & 582 & 1 & $2 A_{1}$ & $2 A_{1}+\underline{A_{1}}+D_{4}$ & 9 & 15 & $\frac{0}{0} \frac{0}{2} \frac{0}{1} \frac{0}{4} \frac{0}{3} \frac{0}{5} \frac{0}{6} \frac{0}{7} \frac{0}{8} \| \frac{4}{2} \frac{5}{0} \frac{5}{3} \frac{2}{2} \frac{2}{8} \frac{3}{b} \frac{3}{c}$ \\
\hline 527 & 1 & $2 A_{1}$ & $4 A_{1}$ & 41 & 114 & $\frac{0}{0} \frac{0}{2} \frac{0}{1} \frac{0}{4} \frac{0}{3} \frac{0}{5} \frac{0}{6} \frac{0}{7} \frac{0}{8} \| \frac{7}{5} \frac{7}{8} \frac{3}{b} \frac{3}{c}$ & 583 & 1 & $2 A_{1}$ & $2 A_{1}+2 \underline{A_{1}}+\ldots A_{3}$ & 12 & 24 & $\frac{0}{0} \frac{0}{2} \frac{0}{1} \frac{0}{4} \frac{0}{3} \frac{0}{5} \frac{0}{6} \frac{0}{7} \frac{0}{8} \| \frac{5}{0} \frac{5}{3} \frac{2}{2} \frac{3}{0} \frac{0}{d} \frac{3}{b} \frac{3}{c}$ \\
\hline 528 & 1 & $2 A_{1}$ & $2 A_{1}+2 \underline{A_{1}}$ & 27 & 72 & $\frac{0}{0} \frac{0}{2} \frac{0}{1} \frac{0}{4} \frac{0}{3} \frac{0}{5} \frac{0}{6} \frac{0}{7} \frac{0}{8} \| \frac{2}{8} \frac{1}{c} \frac{3}{a} \frac{3}{d}$ & 584 & 1 & $2 A_{1}$ & $4 A_{1}+\underline{A_{3}}$ & 10 & 20 & $\frac{0}{0} \frac{0}{2} \frac{0}{1} \frac{0}{4} \frac{0}{3} \frac{0}{5} \frac{0}{6} \frac{0}{7} \frac{0}{8} \| \frac{1}{7} \frac{9}{7} \frac{2}{2} \frac{7}{5} \frac{7}{8} \frac{3}{b} \frac{3}{c}$ \\
\hline 529 & 1 & $2 A_{1}$ & $2 A_{1}+2 \underline{A_{1}}$ & 24 & 72 & $\frac{0}{0} \frac{0}{2} \frac{0}{1} \frac{0}{4} \frac{0}{3} \frac{0}{5} \frac{0}{6} \frac{0}{7} \frac{0}{8} \| \frac{2}{2} \frac{3}{0} \frac{3}{a} \frac{3}{d}$ & 585 & 1 & $2 A_{1}$ & $4 A_{1}+\underline{A_{3}}$ & 8 & 20 & $\frac{0}{0} \frac{0}{2} \frac{0}{1} \frac{0}{4} \frac{0}{3} \frac{0}{5} \frac{0}{6} \frac{0}{7} \frac{0}{8} \| \frac{4}{2} \frac{1}{7} \frac{2}{2} \frac{7}{5} \frac{7}{8} \frac{3}{b} \frac{3}{c}$ \\
\hline 530 & 1 & $2 A_{1}$ & $4 \underline{A_{1}}$ & 17 & 46 & $\frac{0}{0} \frac{0}{2} \frac{0}{1} \frac{0}{4} \frac{0}{3} \frac{0}{5} \frac{0}{6} \frac{0}{7} \frac{0}{8} \| \frac{2}{8} \frac{9}{3} \frac{1}{c} \frac{6}{a}$ & 586 & 1 & $2 A_{1}$ & $4 \underline{A_{1}}+\underline{A_{3}}$ & 8 & 16 & $\frac{0}{0} \frac{0}{2} \frac{0}{1} \frac{0}{4} \frac{0}{3} \frac{0}{5} \frac{0}{6} \frac{0}{7} \frac{0}{8} \| \frac{5}{0} \frac{5}{3} \frac{2}{2} \frac{3}{0} \frac{0}{d} \frac{2}{f} \frac{3}{e}$ \\
\hline 531 & 1 & $2 A_{1}$ & $4 \underline{A_{1}}$ & 16 & 46 & $\frac{0}{0} \frac{0}{2} \frac{0}{1} \frac{0}{4} \frac{0}{3} \frac{0}{5} \frac{0}{6} \frac{0}{7} \frac{0}{8} \| \frac{2}{2} \frac{3}{0} \frac{0}{d} \frac{2}{f}$ & 587 & 1 & $2 A_{1}$ & $2 A_{1}+2 \underline{A_{1}}+\underline{A_{3}}$ & 6 & 12 & $\frac{0}{0} \frac{0}{2} \frac{0}{1} \frac{0}{4} \frac{0}{3} \frac{0}{5} \frac{0}{6} \frac{0}{7} \frac{0}{8} \| \frac{1}{7} \frac{9}{7} \frac{2}{2} \frac{7}{4} \frac{7}{9} \frac{3}{a} \frac{3}{d}$ \\
\hline 532 & 1 & $2 A_{1}$ & $\underline{A_{1}}+\underline{A_{3}}$ & 22 & 54 & $\frac{0}{0} \frac{0}{2} \frac{0}{1} \frac{0}{4} \frac{0}{3} \frac{0}{5} \frac{0}{6} \frac{0}{7} \frac{0}{8} \| \frac{6}{0} \frac{6}{3} \frac{2}{8} \frac{9}{3}$ & 588 & 1 & $2 A_{1}$ & $4 A_{1}+3 \underline{A_{1}}$ & 14 & 32 & $\frac{0}{0} \frac{0}{2} \frac{0}{1} \frac{0}{4} \frac{0}{3} \frac{0}{5} \frac{0}{6} \frac{0}{7} \frac{0}{8} \| \frac{1}{7} \frac{6}{0} \frac{6}{3} \frac{7}{4} \frac{7}{9} \frac{3}{b} \frac{3}{c}$ \\
\hline 533 & 1 & $2 A_{1}$ & $\underline{A_{1}}+\underline{A_{3}}$ & 12 & 26 & $\frac{0}{0} \frac{0}{2} \frac{0}{1} \frac{0}{4} \frac{0}{3} \frac{0}{5} \frac{0}{6} \frac{0}{7} \frac{0}{8} \| \frac{2}{2} \frac{9}{9} \frac{2}{8} \frac{9}{4}$ & 589 & 1 & $2 A_{1}$ & $\underline{A_{1}}+2 \underline{A_{3}}$ & 10 & 18 & $\frac{0}{0} \frac{0}{2} \frac{0}{1} \frac{0}{4} \frac{0}{3} \frac{0}{5} \frac{0}{6} \frac{0}{7} \frac{0}{8} \| \frac{4}{3} \frac{4}{6} \frac{1}{7} \frac{6}{1} \frac{6}{2} \frac{2}{8} \frac{2}{f}$ \\
\hline 534 & 1 & $2 A_{1}$ & $\underline{A_{1}}+\underline{A_{3}}$ & 10 & 26 & $\frac{0}{0} \frac{0}{2} \frac{0}{1} \frac{0}{4} \frac{0}{3} \frac{0}{5} \frac{0}{6} \frac{0}{7} \frac{0}{8} \| \frac{1}{7} \frac{5}{9} \frac{2}{8} \frac{3}{e}$ & 590 & 1 & $2 A_{1}$ & $A_{3}+D_{4}$ & 7 & 12 & $\frac{0}{0} \frac{0}{2} \frac{0}{1} \frac{0}{4} \frac{0}{3} \frac{0}{5} \frac{0}{6} \frac{0}{7} \frac{0}{8} \| \frac{4}{3} \frac{4}{6} \frac{1}{7} \frac{6}{1} \frac{6}{2} \frac{2}{8} \frac{9}{3}$ \\
\hline 535 & 1 & $2 A_{1}$ & $\underline{A_{4}}$ & 7 & 17 & $\frac{0}{0} \frac{0}{2} \frac{0}{1} \frac{0}{4} \frac{0}{3} \frac{0}{5} \frac{0}{6} \frac{0}{7} \frac{0}{8} \| \frac{1}{7} \frac{2}{2} \frac{2}{8} \frac{5}{f}$ & 591 & 1 & $2 A_{1}$ & $\underline{A_{3}}+D_{4}$ & 3 & 6 & $\frac{0}{0} \frac{0}{2} \frac{0}{1} \frac{0}{4} \frac{0}{3} \frac{0}{5} \frac{0}{6} \frac{0}{7} \frac{0}{8} \| \frac{4}{3} \frac{4}{6} \frac{1}{7} \frac{5}{9} \frac{6}{4} \frac{2}{8} \frac{9}{3}$ \\
\hline 536 & 1 & $2 A_{1}$ & $D_{4}$ & 19 & 33 & $\frac{0}{0} \frac{0}{2} \frac{0}{1} \frac{0}{4} \frac{0}{3} \frac{0}{5} \frac{0}{6} \frac{0}{7} \frac{0}{8} \| \frac{7}{3} \frac{6}{0} \frac{6}{3} \frac{2}{8}$ & 592 & 1 & $2 A_{1}$ & $A_{3}+\underline{D_{4}}$ & 3 & 6 & $\frac{0}{0} \frac{0}{2} \frac{0}{1} \frac{0}{4} \frac{0}{3} \frac{0}{5} \frac{0}{6} \frac{0}{7} \frac{0}{8} \| \frac{4}{2} \frac{4}{7} \frac{1}{7} \frac{6}{0} \frac{6}{3} \frac{2}{8} \frac{9}{3}$ \\
\hline 537 & 1 & $2 A_{1}$ & $\underline{D_{4}}$ & 9 & 15 & $\frac{0}{0} \frac{0}{2} \frac{0}{1} \frac{0}{4} \frac{0}{3} \frac{0}{5} \frac{0}{6} \frac{0}{7} \frac{0}{8} \| \frac{2}{2} \frac{7}{3} \frac{9}{9} \frac{2}{8}$ & 593 & 1 & $2 A_{1}$ & $D_{7}$ & 1 & 2 & $\frac{0}{0} \frac{0}{2} \frac{0}{1} \frac{0}{4} \frac{0}{3} \frac{0}{5} \frac{0}{6} \frac{0}{7} \frac{0}{8} \| \frac{1}{0} \frac{1}{3} \frac{4}{2} \frac{1}{7} \frac{2}{2} \frac{7}{3} \frac{2}{8}$ \\
\hline 538 & 1 & $2 A_{1}$ & $2 A_{1}+A_{3}$ & 26 & 54 & $\frac{0}{0} \frac{0}{2} \frac{0}{1} \frac{0}{4} \frac{0}{3} \frac{0}{5} \frac{0}{6} \frac{0}{7} \frac{0}{8} \| \frac{6}{0} \frac{6}{3} \frac{2}{8} \frac{3}{b} \frac{3}{c}$ & 594 & 1 & $2 A_{1}$ & $2 \underline{A_{1}}+2 A_{3}$ & 7 & 12 & $\frac{0}{0} \frac{0}{2} \frac{0}{1} \frac{0}{4} \frac{0}{3} \frac{0}{5} \frac{0}{6} \frac{0}{7} \frac{0}{8} \| \frac{4}{3} \frac{4}{6} \frac{1}{7} \frac{6}{1} \frac{6}{2} \frac{2}{8} \frac{2}{f} \frac{3}{e}$ \\
\hline 539 & 1 & $2 A_{1}$ & $2 \underline{A_{1}}+A_{3}$ & 16 & 36 & $\frac{0}{0} \frac{0}{2} \frac{0}{1} \frac{0}{4} \frac{0}{3} \frac{0}{5} \frac{0}{6} \frac{0}{7} \frac{0}{8} \| \frac{6}{0} \frac{6}{3} \frac{2}{8} \frac{9}{3} \frac{6}{a}$ & 595 & 1 & $2 A_{1}$ & $2 A_{1}+\underline{A_{3}}+A_{3}$ & 5 & 10 & $\frac{0}{0} \frac{0}{2} \frac{0}{1} \frac{0}{4} \frac{0}{3} \frac{0}{5} \frac{0}{6} \frac{0}{7} \frac{0}{8} \| \frac{4}{3} \frac{4}{6} \frac{1}{7} \frac{5}{9} \frac{6}{4} \frac{2}{8} \frac{3}{b} \frac{3}{c}$ \\
\hline 540 & 1 & $2 A_{1}$ & $2 \underline{A_{1}}+A_{3}$ & 15 & 36 & $\frac{0}{0} \frac{0}{2} \frac{0}{1} \frac{0}{4} \frac{0}{3} \frac{0}{5} \frac{0}{6} \frac{0}{7} \frac{0}{8} \| \frac{1}{7} \frac{6}{0} \frac{6}{3} \frac{2}{8} \frac{2}{f}$ & 596 & 1 & $2 A_{1}$ & $2 A_{1}+D_{6}$ & 2 & 4 & $\frac{0}{0} \frac{0}{2} \frac{0}{1} \frac{0}{4} \frac{0}{3} \frac{0}{5} \frac{0}{6} \frac{0}{7} \frac{0}{8} \| \frac{1}{0} \frac{1}{3} \frac{4}{2} \frac{1}{7} \frac{2}{2} \frac{2}{8} \frac{3}{b} \frac{3}{c}$ \\
\hline 541 & 1 & $2 A_{1}$ & $2 A_{1}+\underline{A_{3}}$ & 14 & 28 & $\frac{0}{0} \frac{0}{2} \frac{0}{1} \frac{0}{4} \frac{0}{3} \frac{0}{5} \frac{0}{6} \frac{0}{7} \frac{0}{8} \| \frac{2}{2} \frac{9}{9} \frac{2}{8} \frac{3}{a} \frac{3}{d}$ & 597 & 1 & $2 A_{1}$ & $2 \mathrm{D}_{4}$ & 3 & 5 & $\frac{0}{0} \frac{0}{2} \frac{0}{1} \frac{0}{4} \frac{0}{3} \frac{0}{5} \frac{0}{6} \frac{0}{7} \frac{0}{8} \| \frac{1}{0} \frac{1}{3} \frac{4}{2} \frac{5}{1} \frac{5}{2} \frac{2}{2} \frac{2}{8} \frac{9}{4}$ \\
\hline 542 & 1 & $2 A_{1}$ & $2 A_{1}+\underline{A_{3}}$ & 11 & 28 & $\frac{0}{0} \frac{0}{2} \frac{0}{1} \frac{0}{4} \frac{0}{3} \frac{0}{5} \frac{0}{6} \frac{0}{7} \frac{0}{8} \| \frac{1}{7} \frac{2}{2} \frac{2}{8} \frac{3}{a} \frac{3}{d}$ & 598 & 1 & $2 A_{1}$ & $2 A_{1}+2 \underline{A_{1}}+\ldots D_{4}$ & 6 & 10 & $\frac{0}{0} \frac{0}{2} \frac{0}{1} \frac{0}{4} \frac{0}{3} \frac{0}{5} \frac{0}{6} \frac{0}{7} \frac{0}{8} \| \frac{4}{3} \frac{4}{6} \frac{1}{7} \frac{2}{2} \frac{7}{4} \frac{7}{9} \frac{3}{b} \frac{3}{c}$ \\
\hline 543 & 1 & $2 A_{1}$ & $2 \underline{A_{1}}+\underline{A_{3}}$ & 8 & 18 & $\frac{0}{0} \frac{0}{2} \frac{0}{1} \frac{0}{4} \frac{0}{3} \frac{0}{5} \frac{0}{6} \frac{0}{7} \frac{0}{8} \| \frac{2}{2} \frac{9}{9} \frac{2}{8} \frac{5}{4} \frac{5}{f}$ & 599 & 1 & $2 A_{1}$ & $4 A_{1}+\underline{D_{4}}$ & 4 & 8 & $\frac{0}{0} \frac{0}{2} \frac{0}{1} \frac{0}{4} \frac{0}{3} \frac{0}{5} \frac{0}{6} \frac{0}{7} \frac{0}{8} \| \frac{4}{2} \frac{4}{7} \frac{1}{7} \frac{2}{2} \frac{7}{5} \frac{7}{8} \frac{3}{b} \frac{3}{c}$ \\
\hline 544 & 1 & $2 A_{1}$ & $2 \underline{A_{1}}+\underline{A_{3}}$ & 7 & 18 & $\frac{0}{0} \frac{0}{2} \frac{0}{1} \frac{0}{4} \frac{0}{3} \frac{0}{5} \frac{0}{6} \frac{0}{7} \frac{0}{8} \| \frac{1}{7} \frac{2}{2} \frac{2}{8} \frac{2}{f} \frac{3}{e}$ & 600 & 1 & $2 A_{1}$ & $4 \underline{A_{1}}+4 A_{1}$ & 10 & 21 & $\frac{0}{0} \frac{0}{2} \frac{0}{1} \frac{0}{4} \frac{0}{3} \frac{0}{5} \frac{0}{6} \frac{0}{7} \frac{0}{8} \| \frac{5}{0} \frac{5}{3} \frac{6}{1} \frac{6}{2} \frac{3}{0} \frac{0}{d} \frac{2}{f} \frac{3}{e}$ \\
\hline 545 & 1 & $2 A_{1}$ & $2 A_{1}+\underline{A_{1}}+\underline{A_{2}}$ & 14 & 38 & $\frac{0}{0} \frac{0}{2} \frac{0}{1} \frac{0}{4} \frac{0}{3} \frac{0}{5} \frac{0}{6} \frac{0}{7} \frac{0}{8} \| \frac{1}{7} \frac{5}{9} \frac{2}{8} \frac{3}{a} \frac{3}{d}$ & 601 & 1 & $2 A_{1}$ & $A_{3}+D_{5}$ & 3 & 5 & $\frac{0}{0} \frac{0}{2} \frac{0}{1} \frac{0}{4} \frac{0}{3} \frac{0}{5} \frac{0}{6} \frac{0}{7} \frac{0}{8} \| \frac{1}{0} \frac{1}{3} \frac{4}{2} \frac{1}{7} \frac{6}{1} \frac{6}{2} \frac{2}{8} \frac{9}{3}$ \\
\hline 546 & 1 & $2 A_{1}$ & $4 A_{1}+\underline{A_{1}}$ & 28 & 75 & $\frac{0}{0} \frac{0}{2} \frac{0}{1} \frac{0}{4} \frac{0}{3} \frac{0}{5} \frac{0}{6} \frac{0}{7} \frac{0}{8} \| \frac{2}{2} \frac{7}{5} \frac{7}{8} \frac{3}{b} \frac{3}{c}$ & 602 & 1 & $3 A_{1}$ & $A_{0}$ & 26 & 72 & $\frac{0}{0} \frac{0}{2} \frac{0}{1} \frac{0}{4} \frac{0}{3} \frac{0}{6} \frac{0}{5} \frac{0}{7} \frac{0}{8} \|$ \\
\hline 547 & 1 & $2 A_{1}$ & $2 A_{1}+3 \underline{A_{1}}$ & 18 & 47 & $\frac{0}{0} \frac{0}{2} \frac{0}{1} \frac{0}{4} \frac{0}{3} \frac{0}{5} \frac{0}{6} \frac{0}{7} \frac{0}{8} \| \frac{2}{2} \frac{3}{0} \frac{0}{d} \frac{3}{a} \frac{3}{d}$ & 603 & 1 & $3 A_{1}$ & $\underline{A_{1}}$ & 25 & 48 & $\frac{0}{0} \frac{0}{2} \frac{0}{1} \frac{0}{4} \frac{0}{3} \frac{0}{6} \frac{0}{5} \frac{0}{7} \frac{0}{8} \| \frac{9}{7}$ \\
\hline 548 & 1 & $2 A_{1}$ & $5 \underline{A_{1}}$ & 12 & 31 & $\frac{0}{0} \frac{0}{2} \frac{0}{1} \frac{0}{4} \frac{0}{3} \frac{0}{5} \frac{0}{6} \frac{0}{7} \frac{0}{8} \| \frac{2}{2} \frac{3}{0} \frac{0}{d} \frac{2}{f} \frac{3}{e}$ & 604 & 1 & $3 A_{1}$ & $\underline{A_{1}}$ & 17 & 48 & $\frac{0}{0} \frac{0}{2} \frac{0}{1} \frac{0}{4} \frac{0}{3} \frac{0}{6} \frac{0}{5} \frac{0}{7} \frac{0}{8} \| \frac{2}{8}$ \\
\hline 549 & 1 & $2 A_{1}$ & $\underline{A_{1}}+D_{4}$ & 12 & 22 & $\frac{0}{0} \frac{0}{2} \frac{0}{1} \frac{0}{4} \frac{0}{3} \frac{0}{5} \frac{0}{6} \frac{0}{7} \frac{0}{8} \| \frac{5}{0} \frac{5}{3} \frac{2}{2} \frac{2}{8} \frac{9}{4}$ & 605 & 1 & $3 A_{1}$ & $2 A_{1}$ & 20 & 51 & $\frac{0}{0} \frac{0}{2} \frac{0}{1} \frac{0}{4} \frac{0}{3} \frac{0}{6} \frac{0}{5} \frac{0}{7} \frac{0}{8} \| \frac{7}{5} \frac{7}{8}$ \\
\hline 550 & 1 & $2 A_{1}$ & $\underline{A_{1}}+\underline{D_{4}}$ & 6 & 10 & $\frac{0}{0} \frac{0}{2} \frac{0}{1} \frac{0}{4} \frac{0}{3} \frac{0}{5} \frac{0}{6} \frac{0}{7} \frac{0}{8} \| \frac{1}{7} \frac{9}{7} \frac{2}{2} \frac{2}{8} \frac{2}{f}$ & 606 & 1 & $3 A_{1}$ & $2 \underline{A_{1}}$ & 16 & 33 & $\frac{0}{0} \frac{0}{2} \frac{0}{1} \frac{0}{4} \frac{0}{3} \frac{0}{6} \frac{0}{5} \frac{0}{7} \frac{0}{8} \| \frac{9}{7} \frac{2}{8}$ \\
\hline 551 & 1 & $2 A_{1}$ & $\underline{A_{2}}+\ldots$ & 12 & 30 & $\frac{0}{0} \frac{0}{2} \frac{0}{1} \frac{0}{4} \frac{0}{3} \frac{0}{5} \frac{0}{6} \frac{0}{7} \frac{0}{8} \| \frac{1}{7} \frac{6}{0} \frac{6}{3} \frac{2}{8} \frac{9}{3}$ & 607 & 1 & $3 A_{1}$ & $2 \underline{A_{1}}$ & 12 & 33 & $\frac{0}{0} \frac{0}{2} \frac{0}{1} \frac{0}{4} \frac{0}{3} \frac{0}{6} \frac{0}{5} \frac{0}{7} \frac{0}{8} \| \frac{2}{8} \frac{1}{c}$ \\
\hline 552 & 1 & $2 A_{1}$ & $\underline{A_{2}}+\underline{A_{3}}$ & 6 & 14 & $\frac{0}{0} \frac{0}{2} \frac{0}{1} \frac{0}{4} \frac{0}{3} \frac{0}{5} \frac{0}{6} \frac{0}{7} \frac{0}{8} \| \frac{1}{7} \frac{5}{9} \frac{6}{4} \frac{2}{8} \frac{9}{3}$ & 608 & 1 & $3 A_{1}$ & $A_{2}$ & 25 & 48 & $\frac{0}{0} \frac{0}{2} \frac{0}{1} \frac{0}{4} \frac{0}{3} \frac{0}{6} \frac{0}{5} \frac{0}{7} \frac{0}{8} \| \frac{4}{3} \frac{5}{3}$ \\
\hline 553 & 1 & $2 A_{1}$ & $\underline{A_{5}}$ & 3 & 7 & $\frac{0}{0} \frac{0}{2} \frac{0}{1} \frac{0}{4} \frac{0}{3} \frac{0}{5} \frac{0}{6} \frac{0}{7} \frac{0}{8} \| \frac{4}{2} \frac{1}{7} \frac{2}{2} \frac{2}{8} \frac{3}{e}$ & 609 & 1 & $3 A_{1}$ & $\underline{A_{2}}$ & 9 & 26 & $\frac{0}{0} \frac{0}{2} \frac{0}{1} \frac{0}{4} \frac{0}{3} \frac{0}{6} \frac{0}{5} \frac{0}{7} \frac{0}{8} \| \frac{2}{8} \frac{6}{b}$ \\
\hline 554 & 1 & $2 A_{1}$ & $D_{5}$ & 9 & 14 & $\frac{0}{0} \frac{0}{2} \frac{0}{1} \frac{0}{4} \frac{0}{3} \frac{0}{5} \frac{0}{6} \frac{0}{7} \frac{0}{8} \| \frac{5}{0} \frac{5}{3} \frac{2}{2} \frac{7}{3} \frac{2}{8}$ & 610 & 1 & $3 A_{1}$ & $2 A_{1}+\underline{A_{1}}$ & 19 & 33 & $\frac{0}{0} \frac{0}{2} \frac{0}{1} \frac{0}{4} \frac{0}{3} \frac{0}{6} \frac{0}{5} \frac{0}{7} \frac{0}{8} \| \frac{9}{7} \frac{7}{5} \frac{7}{8}$ \\
\hline
\end{tabular}




\begin{tabular}{|c|c|c|c|c|c|c|c|c|c|c|c|c|c|}
\hline 611 & 1 & $3 A_{1}$ & $2 A_{1}+\underline{A_{1}}$ & 13 & 33 & $\frac{0}{0} \frac{0}{2} \frac{0}{1} \frac{0}{4} \frac{0}{3} \frac{0}{6} \frac{0}{5} \frac{0}{7} \frac{0}{8} \| \frac{2}{8} \frac{1}{d} \frac{2}{c}$ & 667 & 1 & $3 A_{1}$ & $\underline{A_{1}}+A_{5}$ & 6 & 8 & $\frac{0}{0} \frac{0}{2} \frac{0}{1} \frac{0}{4} \frac{0}{3} \frac{0}{6} \frac{0}{5} \frac{0}{7} \frac{0}{8} \| \frac{9}{7} \frac{1}{0} \frac{1}{3} \frac{5}{9} \frac{8}{0} \frac{8}{5}$ \\
\hline 612 & 1 & $3 A_{1}$ & $3 \underline{A_{1}}$ & 11 & 23 & $\frac{0}{0} \frac{0}{2} \frac{0}{1} \frac{0}{4} \frac{0}{3} \frac{0}{6} \frac{0}{5} \frac{0}{7} \frac{0}{8} \| \frac{9}{7} \frac{2}{8} \frac{1}{c}$ & 668 & 1 & $3 A_{1}$ & $\underline{A_{1}}+A_{5}$ & 5 & 8 & $\frac{0}{0} \frac{0}{2} \frac{0}{1} \frac{0}{4} \frac{0}{3} \frac{0}{6} \frac{0}{5} \frac{0}{7} \frac{0}{8} \| \frac{1}{0} \frac{1}{3} \frac{6}{5} \frac{7}{0} \frac{2}{8} \frac{1}{c}$ \\
\hline 613 & 1 & $3 A_{1}$ & $3 \underline{A_{1}}$ & 9 & 23 & $\frac{0}{0} \frac{0}{2} \frac{0}{1} \frac{0}{4} \frac{0}{3} \frac{0}{6} \frac{0}{5} \frac{0}{7} \frac{0}{8} \| \frac{2}{8} \frac{1}{c} \frac{2}{f}$ & 669 & 1 & $3 A_{1}$ & $\underline{A_{1}}+D_{5}$ & 2 & 3 & $\frac{0}{0} \frac{0}{2} \frac{0}{1} \frac{0}{4} \frac{0}{3} \frac{0}{6} \frac{0}{5} \frac{0}{7} \frac{0}{8} \| \frac{9}{7} \frac{1}{0} \frac{1}{3} \frac{5}{9} \frac{7}{3} \frac{2}{8}$ \\
\hline 614 & 1 & $3 A_{1}$ & $\underline{A_{1}}+A_{2}$ & 16 & 33 & $\frac{0}{0} \frac{0}{2} \frac{0}{1} \frac{0}{4} \frac{0}{3} \frac{0}{6} \frac{0}{5} \frac{0}{7} \frac{0}{8} \| \frac{4}{3} \frac{5}{3} \frac{2}{8}$ & 670 & 1 & $3 A_{1}$ & $A_{2}+D_{4}$ & 4 & 8 & $\frac{0}{0} \frac{0}{2} \frac{0}{1} \frac{0}{4} \frac{0}{3} \frac{0}{6} \frac{0}{5} \frac{0}{7} \frac{0}{8} \| \frac{4}{3} \frac{5}{3} \frac{5}{9} \frac{6}{8} \frac{7}{1} \frac{2}{8}$ \\
\hline 615 & 1 & $3 A_{1}$ & $\underline{A_{1}}+\underline{A_{2}}$ & 8 & 19 & $\frac{0}{0} \frac{0}{2} \frac{0}{1} \frac{0}{4} \frac{0}{3} \frac{0}{6} \frac{0}{5} \frac{0}{7} \frac{0}{8} \| \frac{9}{7} \frac{2}{8} \frac{6}{b}$ & 671 & 1 & $3 A_{1}$ & $A_{2}+\underline{D_{4}}$ & 2 & 4 & $\frac{0}{0} \frac{0}{2} \frac{0}{1} \frac{0}{4} \frac{0}{3} \frac{0}{6} \frac{0}{5} \frac{0}{7} \frac{0}{8} \| \frac{4}{3} \frac{5}{3} \frac{5}{9} \frac{6}{4} \frac{7}{3} \frac{2}{8}$ \\
\hline 616 & 1 & $3 A_{1}$ & $A_{3}$ & 11 & 27 & $\frac{0}{0} \frac{0}{2} \frac{0}{1} \frac{0}{4} \frac{0}{3} \frac{0}{6} \frac{0}{5} \frac{0}{7} \frac{0}{8} \| \frac{5}{9} \frac{8}{2} \frac{8}{7}$ & 672 & 1 & $3 A_{1}$ & $E_{6}$ & 3 & 3 & $\frac{0}{0} \frac{0}{2} \frac{0}{1} \frac{0}{4} \frac{0}{3} \frac{0}{6} \frac{0}{5} \frac{0}{7} \frac{0}{8} \| \frac{1}{0} \frac{1}{3} \frac{1}{4} \frac{1}{9} \frac{5}{9} \frac{2}{8}$ \\
\hline 617 & 1 & $3 A_{1}$ & $\underline{A_{3}}$ & 5 & 13 & $\frac{0}{0} \frac{0}{2} \frac{0}{1} \frac{0}{4} \frac{0}{3} \frac{0}{6} \frac{0}{5} \frac{0}{7} \frac{0}{8} \| \frac{5}{9} \frac{2}{8} \frac{3}{e}$ & 673 & 1 & $3 A_{1}$ & $2 \underline{A_{1}}+A_{2}+\ldots A_{3}$ & 5 & 8 & $\frac{0}{0} \frac{0}{2} \frac{0}{1} \frac{0}{4} \frac{0}{3} \frac{0}{6} \frac{0}{5} \frac{0}{7} \frac{0}{8} \| \frac{4}{3} \frac{5}{3} \frac{6}{1} \frac{6}{2} \frac{2}{8} \frac{2}{f} \frac{3}{e}$ \\
\hline 618 & 1 & $3 A_{1}$ & $2 A_{1}+A_{2}$ & 19 & 33 & $\frac{0}{0} \frac{0}{2} \frac{0}{1} \frac{0}{4} \frac{0}{3} \frac{0}{6} \frac{0}{5} \frac{0}{7} \frac{0}{8} \| \frac{4}{3} \frac{5}{3} \frac{7}{6} \frac{7}{7}$ & 674 & 1 & $3 A_{1}$ & $2 A_{1}+A_{2}+\underline{A_{3}}$ & 3 & 6 & $\frac{0}{0} \frac{0}{2} \frac{0}{1} \frac{0}{4} \frac{0}{3} \frac{0}{6} \frac{0}{5} \frac{0}{7} \frac{0}{8} \| \frac{4}{3} \frac{5}{3} \frac{5}{9} \frac{6}{4} \frac{2}{8} \frac{3}{b} \frac{3}{c}$ \\
\hline 619 & 1 & $3 A_{1}$ & $2 \underline{A_{1}}+A_{2}$ & 11 & 23 & $\frac{0}{0} \frac{0}{2} \frac{0}{1} \frac{0}{4} \frac{0}{3} \frac{0}{6} \frac{0}{5} \frac{0}{7} \frac{0}{8} \| \frac{4}{3} \frac{5}{3} \frac{2}{8} \frac{1}{c}$ & 675 & 1 & $3 A_{1}$ & $2 \underline{A_{1}}+A_{5}$ & 4 & 5 & $\frac{0}{0} \frac{0}{2} \frac{0}{1} \frac{0}{4} \frac{0}{3} \frac{0}{6} \frac{0}{5} \frac{0}{7} \frac{0}{8} \| \frac{9}{7} \frac{1}{0} \frac{1}{3} \frac{5}{9} \frac{7}{3} \frac{8}{0} \frac{8}{5}$ \\
\hline 620 & 1 & $3 A_{1}$ & $2 A_{1}+\underline{A_{2}}$ & 7 & 17 & $\frac{0}{0} \frac{0}{2} \frac{0}{1} \frac{0}{4} \frac{0}{3} \frac{0}{6} \frac{0}{5} \frac{0}{7} \frac{0}{8} \| \frac{5}{9} \frac{2}{8} \frac{1}{f} \frac{2}{e}$ & 676 & 1 & $3 A_{1}$ & $2 A_{1}+\underline{A_{1}}+D_{4}$ & 3 & 5 & $\frac{0}{0} \frac{0}{2} \frac{0}{1} \frac{0}{4} \frac{0}{3} \frac{0}{6} \frac{0}{5} \frac{0}{7} \frac{0}{8} \| \frac{9}{7} \frac{1}{0} \frac{1}{3} \frac{5}{9} \frac{2}{8} \frac{3}{b} \frac{3}{c}$ \\
\hline 621 & 1 & $3 A_{1}$ & $2 A_{2}$ & 14 & 31 & $\frac{0}{0} \frac{0}{2} \frac{0}{1} \frac{0}{4} \frac{0}{3} \frac{0}{6} \frac{0}{5} \frac{0}{7} \frac{0}{8} \| \frac{6}{0} \frac{6}{3} \frac{8}{0} \frac{8}{5}$ & 677 & 1 & $3 A_{1}$ & $4 A_{1}+3 \underline{A_{1}}$ & 6 & 10 & $\frac{0}{0} \frac{0}{2} \frac{0}{1} \frac{0}{4} \frac{0}{3} \frac{0}{6} \frac{0}{5} \frac{0}{7} \frac{0}{8} \| \frac{9}{7} \frac{6}{0} \frac{6}{3} \frac{7}{4} \frac{7}{9} \frac{3}{b} \frac{3}{c}$ \\
\hline 622 & 1 & $3 A_{1}$ & $\underline{A_{2}}+\ldots$ & 8 & 19 & $\frac{0}{0} \frac{0}{2} \frac{0}{1} \frac{0}{4} \frac{0}{3} \frac{0}{6} \frac{0}{5} \frac{0}{7} \frac{0}{8} \| \frac{4}{3} \frac{5}{3} \frac{2}{8} \frac{6}{b}$ & 678 & 1 & $3 A_{1}$ & $\underline{A_{1}}+2 A_{3}$ & 8 & 10 & $\frac{0}{0} \frac{0}{2} \frac{0}{1} \frac{0}{4} \frac{0}{3} \frac{0}{6} \frac{0}{5} \frac{0}{7} \frac{0}{8} \| \frac{9}{7} \frac{1}{0} \frac{1}{3} \frac{6}{1} \frac{6}{2} \frac{8}{0} \frac{8}{5}$ \\
\hline 623 & 1 & $3 A_{1}$ & $4 A_{1}$ & 15 & 36 & $\frac{0}{0} \frac{0}{2} \frac{0}{1} \frac{0}{4} \frac{0}{3} \frac{0}{6} \frac{0}{5} \frac{0}{7}\|\|_{5} \frac{7}{8} \frac{3}{b} \frac{3}{c}$ & 679 & 1 & $3 A_{1}$ & $\underline{A_{1}}+2 A_{3}$ & 6 & 10 & $\frac{0}{0} \frac{0}{2} \frac{0}{1} \frac{0}{4} \frac{0}{3} \frac{0}{6} \frac{0}{5} \frac{0}{7} \frac{0}{8} \| \frac{1}{0} \frac{1}{3} \frac{6}{5} \frac{7}{0} \frac{8}{8} \frac{7}{6}$ \\
\hline 624 & 1 & $3 A_{1}$ & $2 A_{1}+2 \underline{A_{1}}$ & 12 & 22 & $\frac{0}{0} \frac{0}{2} \frac{0}{1} \frac{0}{4} \frac{0}{3} \frac{0}{6} \frac{0}{5} \frac{0}{7} \frac{0}{8} \| \frac{9}{7} \frac{2}{8} \frac{1}{d} \frac{2}{c}$ & 680 & 1 & $3 A_{1}$ & $\underline{A_{1}}+2 A_{3}$ & 4 & 6 & $\frac{0}{0} \frac{0}{2} \frac{0}{1} \frac{0}{4} \frac{0}{3} \frac{0}{6} \frac{0}{5} \frac{0}{7} \frac{0}{8} \| \frac{9}{7} \frac{1}{0} \frac{1}{3} \frac{5}{9} \frac{7}{3} \frac{7}{6} \frac{7}{7}$ \\
\hline 625 & 1 & $3 A_{1}$ & $2 A_{1}+2 \underline{A_{1}}$ & 9 & 22 & $\frac{0}{0} \frac{0}{2} \frac{0}{1} \frac{0}{4} \frac{0}{3} \frac{0}{6} \frac{0}{5} \frac{0}{7} \frac{0}{8} \| \frac{2}{8} \frac{1}{c} \frac{3}{a} \frac{3}{d}$ & 681 & 1 & $3 A_{1}$ & $\underline{A_{1}}+2 A_{2}+A_{2}$ & 8 & 12 & $\frac{0}{0} \frac{0}{2} \frac{0}{1} \frac{0}{4} \frac{0}{3} \frac{0}{6} \frac{0}{5} \frac{0}{7} \frac{0}{8} \| \frac{4}{3} \frac{5}{3} \frac{6}{1} \frac{6}{2} \frac{7}{4} \frac{8}{3} \frac{8}{6}$ \\
\hline 626 & 1 & $3 A_{1}$ & $4 \underline{A_{1}}$ & 8 & 16 & $\frac{0}{0} \frac{0}{2} \frac{0}{1} \frac{0}{4} \frac{0}{3} \frac{0}{6} \frac{0}{5} \frac{0}{7} \frac{0}{8} \| \frac{9}{7} \frac{2}{8} \frac{1}{c} \frac{2}{f}$ & 682 & 1 & $3 A_{1}$ & $\underline{A_{1}}+2 A_{2}+\underline{A_{2}}$ & 4 & 6 & $\frac{0}{0} \frac{0}{2} \frac{0}{1} \frac{0}{4} \frac{0}{3} \frac{0}{6} \frac{0}{5} \frac{0}{7} \frac{0}{8} \| \frac{9}{7} \frac{5}{9} \frac{6}{7} \frac{7}{2} \frac{7}{9} \frac{8}{0} \frac{8}{5}$ \\
\hline 627 & 1 & $3 A_{1}$ & $4 \underline{A_{1}}$ & 7 & 16 & $\frac{0}{0} \frac{0}{2} \frac{0}{1} \frac{0}{4} \frac{0}{3} \frac{0}{6} \frac{0}{5} \frac{0}{7} \frac{0}{8} \| \frac{2}{8} \frac{1}{c} \frac{2}{f} \frac{3}{e}$ & 683 & 1 & $3 A_{1}$ & $\underline{A_{1}}+E_{6}$ & 2 & 2 & $\frac{0}{0} \frac{0}{2} \frac{0}{1} \frac{0}{4} \frac{0}{3} \frac{0}{6} \frac{0}{5} \frac{0}{7} \frac{0}{8} \| \frac{9}{7} \frac{1}{0} \frac{1}{3} \frac{1}{4} \frac{1}{9} \frac{5}{9} \frac{2}{8}$ \\
\hline 628 & 1 & $3 A_{1}$ & $\underline{A_{1}}+A_{3}$ & 10 & 18 & $\frac{0}{0} \frac{0}{2} \frac{0}{1} \frac{0}{4} \frac{0}{3} \frac{0}{6} \frac{0}{5} \frac{0}{7} \frac{0}{8} \| \frac{9}{7} \frac{5}{9} \frac{8}{2} \frac{8}{7}$ & 684 & 1 & $3 A_{1}$ & $A_{2}+A_{5}$ & 6 & 8 & $\frac{0}{0} \frac{0}{2} \frac{0}{1} \frac{0}{4} \frac{0}{3} \frac{0}{6} \frac{0}{5} \frac{0}{7} \frac{0}{8} \| \frac{1}{0} \frac{1}{3} \frac{4}{4} \frac{5}{2} \frac{5}{9} \frac{8}{0} \frac{8}{5}$ \\
\hline 629 & 1 & $3 A_{1}$ & $\underline{A_{1}}+A_{3}$ & 8 & 18 & $\frac{0}{0} \frac{0}{2} \frac{0}{1} \frac{0}{4} \frac{0}{3} \frac{0}{6} \frac{0}{5} \frac{0}{7} \frac{0}{8} \| \frac{6}{0} \frac{6}{3} \frac{2}{8} \frac{2}{f}$ & 685 & 1 & $3 A_{1}$ & $A_{2}+D_{5}$ & 2 & 3 & $\frac{0}{0} \frac{0}{2} \frac{0}{1} \frac{0}{4} \frac{0}{3} \frac{0}{6} \frac{0}{5} \frac{0}{7} \frac{0}{8} \| \frac{1}{0} \frac{1}{3} \frac{4}{4} \frac{5}{2} \frac{5}{9} \frac{7}{3} \frac{2}{8}$ \\
\hline 630 & 1 & $3 A_{1}$ & $\underline{A_{1}}+\underline{A_{3}}$ & 4 & 10 & $\frac{0}{0} \frac{0}{2} \frac{0}{1} \frac{0}{4} \frac{0}{3} \frac{0}{6} \frac{0}{5} \frac{0}{7} \frac{0}{8} \| \frac{9}{7} \frac{5}{9} \frac{2}{8} \frac{3}{e}$ & 686 & 1 & $3 A_{1}$ & $A_{7}$ & 4 & 5 & $\frac{0}{0} \frac{0}{2} \frac{0}{1} \frac{0}{4} \frac{0}{3} \frac{0}{6} \frac{0}{5} \frac{0}{7} \frac{0}{8} \| \frac{1}{0} \frac{1}{3} \frac{1}{4} \frac{1}{9} \frac{5}{9} \frac{7}{6} \frac{7}{7}$ \\
\hline 631 & 1 & $3 A_{1}$ & $D_{4}$ & 5 & 11 & $\frac{0}{0} \frac{0}{2} \frac{0}{1} \frac{0}{4} \frac{0}{3} \frac{0}{6} \frac{0}{5} \frac{0}{7} \frac{0}{8} \| \frac{5}{9} \frac{6}{7} \frac{7}{2} \frac{2}{8}$ & 687 & 1 & $3 A_{1}$ & $2 \underline{A_{1}}+2 A_{3}$ & 5 & 6 & $\frac{0}{0} \frac{0}{2} \frac{0}{1} \frac{0}{4} \frac{0}{3} \frac{0}{6} \frac{0}{5} \frac{0}{7} \frac{0}{8} \| \frac{9}{7} \frac{1}{0} \frac{1}{3} \frac{6}{5} \frac{7}{0} \frac{8}{8} \frac{7}{6} \frac{7}{7}$ \\
\hline 632 & 1 & $3 A_{1}$ & $\underline{D_{4}}$ & 3 & 5 & $\frac{0}{0} \frac{0}{2} \frac{0}{1} \frac{0}{4} \frac{0}{3} \frac{0}{6} \frac{0}{5} \frac{0}{7} \frac{0}{8} \| \frac{5}{9} \frac{6}{4} \frac{7}{3} \frac{2}{8}$ & 688 & 1 & $3 A_{1}$ & $2 A_{2}+\underline{A_{2}}+A_{2}$ & 4 & 6 & $\frac{0}{0} \frac{0}{2} \frac{0}{1} \frac{0}{4} \frac{0}{3} \frac{0}{6} \frac{0}{5} \frac{0}{7} \frac{0}{8} \| \frac{4}{3} \frac{5}{3} \frac{5}{9} \frac{6}{8} \frac{7}{1} \frac{7}{9} \frac{8}{1} \frac{8}{4}$ \\
\hline 633 & 1 & $3 A_{1}$ & $2 A_{1}+A_{3}$ & 8 & 18 & $\frac{0}{0} \frac{0}{2} \frac{0}{1} \frac{0}{4} \frac{0}{3} \frac{0}{6} \frac{0}{5} \frac{0}{7} \frac{0}{8} \| \frac{6}{0} \frac{6}{3} \frac{2}{8} \frac{3}{b} \frac{3}{c}$ & 689 & 1 & $3 A_{1}$ & $\underline{A_{1}}+A_{2}+A_{5}$ & 4 & 5 & $\frac{0}{0} \frac{0}{2} \frac{0}{1} \frac{0}{4} \frac{0}{3} \frac{0}{6} \frac{0}{5} \frac{0}{7} \frac{0}{8} \| \frac{1}{0} \frac{1}{3} \frac{4}{4} \frac{5}{2} \frac{6}{5} \frac{7}{0} \frac{2}{8} \frac{1}{c}$ \\
\hline 634 & 1 & $3 A_{1}$ & $2 \underline{A_{1}}+A_{3}$ & 7 & 12 & $\frac{0}{0} \frac{0}{2} \frac{0}{1} \frac{0}{4} \frac{0}{3} \frac{0}{6} \frac{0}{5} \frac{0}{7} \frac{0}{8} \| \frac{9}{7} \frac{6}{0} \frac{6}{3} \frac{2}{8} \frac{2}{f}$ & 690 & 1 & $3 A_{1}$ & $\underline{A_{1}}+A_{7}$ & 3 & 3 & $\frac{0}{0} \frac{0}{2} \frac{0}{1} \frac{0}{4} \frac{0}{3} \frac{0}{6} \frac{0}{5} \frac{0}{7} \frac{0}{8} \| \frac{9}{7} \frac{1}{0} \frac{1}{3} \frac{1}{4} \frac{1}{9} \frac{5}{9} \frac{7}{6} \frac{7}{7}$ \\
\hline 635 & 1 & $3 A_{1}$ & $2 \underline{A_{1}}+A_{3}$ & 6 & 12 & $\frac{0}{0} \frac{0}{2} \frac{0}{1} \frac{0}{4} \frac{0}{3} \frac{0}{6} \frac{0}{5} \frac{0}{7} \frac{0}{8} \| \frac{6}{0} \frac{6}{3} \frac{2}{8} \frac{2}{f} \frac{3}{e}$ & 691 & 1 & $3 A_{1}$ & $A_{2}+E_{6}$ & 2 & 2 & $\frac{0}{0} \frac{0}{2} \frac{0}{1} \frac{0}{4} \frac{0}{3} \frac{0}{6} \frac{0}{5} \frac{0}{7} \frac{0}{8} \| \frac{1}{0} \frac{1}{3} \frac{1}{4} \frac{1}{9} \frac{4}{5} \frac{5}{1} \frac{5}{9} \frac{2}{8}$ \\
\hline 636 & 1 & $3 A_{1}$ & $2 A_{1}+\underline{A_{3}}$ & 4 & 8 & $\frac{0}{0} \frac{0}{2} \frac{0}{1} \frac{0}{4} \frac{0}{3} \frac{0}{6} \frac{0}{5} \frac{0}{7} \frac{0}{8} \| \frac{5}{9} \frac{6}{4} \frac{2}{8} \frac{3}{a} \frac{3}{d}$ & 692 & 1 & $4 A_{1}$ & $A_{0}$ & 8 & 24 & $\frac{0}{0} \frac{0}{2} \frac{0}{1} \frac{0}{4} \frac{0}{3} \frac{0}{6} \frac{0}{5} \frac{0}{7} \|$ \\
\hline 637 & 1 & $3 A_{1}$ & $2 A_{1}+\underline{A_{1}}+\underline{A_{2}}$ & 12 & 22 & $\frac{0}{0} \frac{0}{2} \frac{0}{1} \frac{0}{4} \frac{0}{3} \frac{0}{6} \frac{0}{5} \frac{0}{7} \frac{0}{8} \| \frac{4}{3} \frac{5}{3} \frac{2}{8} \frac{1}{e} \frac{2}{b}$ & 693 & 1 & $4 A_{1}$ & $\underline{A_{1}}$ & 7 & 18 & $\frac{0}{0} \frac{0}{2} \frac{0}{1} \frac{0}{4} \frac{0}{3} \frac{0}{6} \frac{0}{5} \frac{0}{8}\left\|_{7}\right\| \frac{9}{7}$ \\
\hline 638 & 1 & $3 A_{1}$ & $3 \underline{A_{1}}+A_{2}$ & 8 & 16 & $\frac{0}{0} \frac{0}{2} \frac{0}{1} \frac{0}{4} \frac{0}{3} \frac{0}{6} \frac{0}{5} \frac{0}{7} \frac{0}{8} \| \frac{4}{3} \frac{5}{3} \frac{2}{8} \frac{1}{c} \frac{2}{f}$ & 694 & 1 & $4 A_{1}$ & $2 A_{1}$ & 6 & 15 & $\frac{0}{0} \frac{0}{2} \frac{0}{1} \frac{0}{4} \frac{0}{3} \frac{0}{6} \frac{0}{5} \frac{0}{8}\|\|_{0} \frac{1}{3}$ \\
\hline 639 & 1 & $3 A_{1}$ & $2 A_{1}+\underline{A_{1}}+\underline{A_{2}}$ & 6 & 12 & $\frac{0}{0} \frac{0}{2} \frac{0}{1} \frac{0}{4} \frac{0}{3} \frac{0}{6} \frac{0}{5} \frac{0}{7} \frac{0}{8} \| \frac{9}{7} \frac{5}{9} \frac{2}{8} \frac{1}{f} \frac{2}{e}$ & 695 & 1 & $4 A_{1}$ & $2 \underline{A_{1}}$ & 6 & 13 & $\frac{0}{0} \frac{0}{2} \frac{0}{1} \frac{0}{4} \frac{0}{3} \frac{0}{6} \frac{0}{5} \frac{0}{8} \frac{0}{7} \| \frac{9}{7} \frac{1}{c}$ \\
\hline 640 & 1 & $3 A_{1}$ & $4 A_{1}+\underline{A_{1}}$ & 14 & 23 & $\frac{0}{0} \frac{0}{2} \frac{0}{1} \frac{0}{4} \frac{0}{3} \frac{0}{6} \frac{0}{5} \frac{0}{7} \frac{0}{8} \| \frac{9}{7} \frac{7}{5} \frac{7}{8} \frac{3}{b} \frac{3}{c}$ & 696 & 1 & $4 A_{1}$ & $A_{2}$ & 7 & 18 & $\frac{0}{0} \frac{0}{2} \frac{0}{1} \frac{0}{4} \frac{0}{3} \frac{0}{6} \frac{0}{5} \frac{0}{8} \frac{0}{7} \| \frac{4}{3} \frac{5}{3}$ \\
\hline 641 & 1 & $3 A_{1}$ & $4 A_{1}+\underline{A_{1}}$ & 10 & 23 & $\frac{0}{0} \frac{0}{2} \frac{0}{1} \frac{0}{4} \frac{0}{3} \frac{0}{6} \frac{0}{5} \frac{0}{7} \frac{0}{8} \| \frac{5}{9} \frac{7}{5} \frac{7}{8} \frac{3}{b} \frac{3}{c}$ & 697 & 1 & $4 A_{1}$ & $2 A_{1}+\underline{A_{1}}$ & 5 & 11 & $\frac{0}{0} \frac{0}{2} \frac{0}{1} \frac{0}{4} \frac{0}{3} \frac{0}{6} \frac{0}{5} \frac{0}{8} \| \frac{9}{7} \frac{1}{0} \frac{1}{3}$ \\
\hline 642 & 1 & $3 A_{1}$ & $2 A_{1}+3 \underline{A_{1}}$ & 8 & 15 & $\frac{0}{0} \frac{0}{2} \frac{0}{1} \frac{0}{4} \frac{0}{3} \frac{0}{6} \frac{0}{5} \frac{0}{7} \frac{0}{8} \| \frac{9}{7} \frac{2}{8} \frac{1}{c} \frac{3}{a} \frac{3}{d}$ & 698 & 1 & $4 A_{1}$ & $3 \underline{A_{1}}$ & 5 & 9 & $\frac{0}{0} \frac{0}{2} \frac{0}{1} \frac{0}{4} \frac{0}{3} \frac{0}{6} \frac{0}{5} \frac{0}{8} \frac{0}{7} \| \frac{9}{7} \frac{1}{c} \frac{2}{f}$ \\
\hline 643 & 1 & $3 A_{1}$ & $5 \underline{A_{1}}$ & 6 & 11 & $\frac{0}{0} \frac{0}{2} \frac{0}{1} \frac{0}{4} \frac{0}{3} \frac{0}{6} \frac{0}{5} \frac{0}{7} \frac{0}{8} \| \frac{9}{7} \frac{2}{8} \frac{1}{c} \frac{2}{f} \frac{3}{e}$ & 699 & 1 & $4 A_{1}$ & $\underline{A_{1}}+A_{2}$ & 6 & 13 & $\frac{0}{0} \frac{0}{2} \frac{0}{1} \frac{0}{4} \frac{0}{3} \frac{0}{6} \frac{0}{5} \frac{0}{8} \| \frac{9}{7} \frac{6}{0} \frac{7}{8}$ \\
\hline 644 & 1 & $3 A_{1}$ & $\underline{A_{1}}+2 A_{2}$ & 13 & 19 & $\frac{0}{0} \frac{0}{2} \frac{0}{1} \frac{0}{4} \frac{0}{3} \frac{0}{6} \frac{0}{5} \frac{0}{7} \frac{0}{8} \| \frac{9}{7} \frac{6}{0} \frac{6}{3} \frac{8}{0} \frac{8}{5}$ & 700 & 1 & $4 A_{1}$ & $A_{3}$ & 5 & 9 & $\frac{0}{0} \frac{0}{2} \frac{0}{1} \frac{0}{4} \frac{0}{3} \frac{0}{6} \frac{0}{5} \frac{0}{8} \frac{0}{7} \| \frac{9}{7} \frac{2}{2} \frac{3}{0}$ \\
\hline 645 & 1 & $3 A_{1}$ & $\underline{A_{1}}+2 A_{2}$ & 9 & 19 & $\frac{0}{0} \frac{0}{2} \frac{0}{1} \frac{0}{4} \frac{0}{3} \frac{0}{6} \frac{0}{5} \frac{0}{7} \frac{0}{8} \| \frac{6}{0} \frac{6}{3} \frac{7}{4} \frac{8}{2} \frac{8}{7}$ & 701 & 1 & $4 A_{1}$ & $2 A_{1}+A_{2}$ & 5 & 11 & $\frac{0}{0} \frac{0}{2} \frac{0}{1} \frac{0}{4} \frac{0}{3} \frac{0}{6} \frac{0}{5} \frac{0}{8} \frac{0}{7} \| \frac{1}{0} \frac{1}{3} \frac{4}{4} \frac{5}{2}$ \\
\hline 646 & 1 & $3 A_{1}$ & $\underline{A_{1}}+D_{4}$ & 4 & 8 & $\frac{0}{0} \frac{0}{2} \frac{0}{1} \frac{0}{4} \frac{0}{3} \frac{0}{6} \frac{0}{5} \frac{0}{7} \frac{0}{8} \| \frac{9}{7} \frac{5}{9} \frac{6}{7} \frac{7}{2} \frac{2}{8}$ & 702 & 1 & $4 A_{1}$ & $2 \underline{A_{1}}+\underline{A_{2}}$ & 5 & 9 & $\frac{0}{0} \frac{0}{2} \frac{0}{1} \frac{0}{4} \frac{0}{3} \frac{0}{6} \frac{0}{5} \frac{0}{8} \frac{0}{7} \| \frac{9}{7} \frac{1}{c} \frac{6}{5} \frac{8}{5}$ \\
\hline 647 & 1 & $3 A_{1}$ & $\underline{A_{1}}+\underline{D_{4}}$ & 2 & 4 & $\frac{0}{0} \frac{0}{2} \frac{0}{1} \frac{0}{4} \frac{0}{3} \frac{0}{6} \frac{0}{5} \frac{0}{7} \frac{0}{8} \| \frac{9}{7} \frac{5}{9} \frac{6}{4} \frac{7}{3} \frac{2}{8}$ & 703 & 1 & $4 A_{1}$ & $2 A_{2}$ & 6 & 13 & $\frac{0}{0} \frac{0}{2} \frac{0}{1} \frac{0}{4} \frac{0}{3} \frac{0}{6} \frac{0}{5} \frac{0}{8} \frac{0}{7} \| \frac{4}{3} \frac{5}{3} \frac{6}{1} \frac{7}{7}$ \\
\hline 648 & 1 & $3 A_{1}$ & $A_{2}+A_{3}$ & 10 & 18 & $\frac{0}{0} \frac{0}{2} \frac{0}{1} \frac{0}{4} \frac{0}{3} \frac{0}{6} \frac{0}{5} \frac{0}{7} \frac{0}{8} \| \frac{4}{3} \frac{5}{3} \frac{5}{9} \frac{8}{3} \frac{8}{6}$ & 704 & 1 & $4 A_{1}$ & $2 A_{2}$ & 4 & 7 & $\frac{0}{0} \frac{0}{2} \frac{0}{1} \frac{0}{4} \frac{0}{3} \frac{0}{6} \frac{0}{5} \frac{0}{8} \frac{0}{7} \| \frac{1}{0} \frac{1}{3} \frac{1}{4} \frac{1}{9}$ \\
\hline 649 & 1 & $3 A_{1}$ & $A_{2}+\underline{A_{3}}$ & 4 & 10 & $\frac{0}{0} \frac{0}{2} \frac{0}{1} \frac{0}{4} \frac{0}{3} \frac{0}{6} \frac{0}{5} \frac{0}{7} \frac{0}{8} \| \frac{4}{3} \frac{5}{3} \frac{5}{9} \frac{2}{e}$ & 705 & 1 & $4 A_{1}$ & $4 A_{1}$ & 5 & 10 & $\frac{0}{0} \frac{0}{2} \frac{0}{1} \frac{0}{4} \frac{0}{3} \frac{0}{6} \frac{0}{5} \frac{0}{8} \frac{0}{7} \| \frac{1}{0} \frac{1}{3} \frac{3}{b} \frac{3}{c}$ \\
\hline 650 & 1 & $3 A_{1}$ & $A_{5}$ & 7 & 13 & $\frac{0}{0} \frac{0}{2} \frac{0}{1} \frac{0}{4} \frac{0}{3} \frac{0}{6} \frac{0}{5} \frac{0}{7} \frac{0}{8} \| \frac{1}{0} \frac{1}{3} \frac{5}{9} \frac{8}{0} \frac{8}{5}$ & 706 & 1 & $4 A_{1}$ & $4 A_{1}$ & 4 & 10 & $\frac{0}{0} \frac{0}{2} \frac{0}{1} \frac{0}{4} \frac{0}{3} \frac{0}{6} \frac{0}{5} \frac{0}{8} \frac{0}{7} \| \frac{1}{0} \frac{1}{3} \frac{2}{2} \frac{3}{0}$ \\
\hline 651 & 1 & $3 A_{1}$ & $D_{5}$ & 3 & 4 & $\frac{0}{0} \frac{0}{2} \frac{0}{1} \frac{0}{4} \frac{0}{3} \frac{0}{6} \frac{0}{5} \frac{0}{7} \frac{0}{8} \| \frac{1}{0} \frac{1}{3} \frac{5}{9} \frac{7}{3} \frac{2}{8}$ & 707 & 1 & $4 A_{1}$ & $2 A_{1}+2 \underline{A_{1}}$ & 4 & 8 & $\frac{0}{0} \frac{0}{2} \frac{0}{1} \frac{0}{4} \frac{0}{3} \frac{0}{6} \frac{0}{5} \frac{0}{8} \frac{0}{7} \| \frac{9}{7} \frac{1}{c} \frac{1}{0} \frac{1}{3}$ \\
\hline 652 & 1 & $3 A_{1}$ & $2 \underline{A_{1}}+2 A_{2}$ & 8 & 12 & $\frac{0}{0} \frac{0}{2} \frac{0}{1} \frac{0}{4} \frac{0}{3} \frac{0}{6} \frac{0}{5} \frac{0}{7} \frac{0}{8} \| \frac{9}{7} \frac{6}{0} \frac{6}{3} \frac{7}{4} \frac{8}{2} \frac{8}{7}$ & 708 & 1 & $4 A_{1}$ & $4 \underline{A_{1}}$ & 4 & 6 & $\frac{0}{0} \frac{0}{2} \frac{0}{1} \frac{0}{4} \frac{0}{3} \frac{0}{6} \frac{0}{5} \frac{0}{8} \frac{0}{7} \| \frac{9}{7} \frac{1}{c} \frac{2}{f} \frac{3}{e}$ \\
\hline 653 & 1 & $3 A_{1}$ & $2 A_{1}+\underline{A_{2}}+A_{2}$ & 6 & 12 & $\frac{0}{0} \frac{0}{2} \frac{0}{1} \frac{0}{4} \frac{0}{3} \frac{0}{6} \frac{0}{5} \frac{0}{7} \frac{0}{8} \| \frac{4}{3} \frac{5}{3} \frac{5}{9} \frac{2}{8} \frac{2}{d}$ & 709 & 1 & $4 A_{1}$ & $\underline{A_{1}}+A_{3}$ & 4 & 6 & $\frac{0}{0} \frac{0}{2} \frac{0}{1} \frac{0}{4} \frac{0}{3} \frac{0}{6} \frac{0}{5} \frac{0}{8} \frac{0}{7} \| \frac{9}{7} \frac{1}{c} \frac{1}{4} \frac{1}{9}$ \\
\hline 654 & 1 & $3 A_{1}$ & $2 A_{1}+D_{4}$ & 4 & 7 & $\frac{0}{0} \frac{0}{2} \frac{0}{1} \frac{0}{4} \frac{0}{3} \frac{0}{6} \frac{0}{5} \frac{0}{7} \frac{0}{8} \| \frac{1}{0} \frac{1}{3} \frac{5}{9} \frac{2}{8} \frac{3}{b} \frac{3}{c}$ & 710 & 1 & $4 A_{1}$ & $A_{4}$ & 5 & 9 & $\frac{0}{0} \frac{0}{2} \frac{0}{1} \frac{0}{4} \frac{0}{3} \frac{0}{6} \frac{0}{5} \frac{0}{8} \frac{0}{7} \| \frac{1}{0} \frac{1}{3} \frac{6}{5} \frac{8}{5}$ \\
\hline 655 & 1 & $3 A_{1}$ & $2 A_{3}$ & 9 & 17 & $\frac{0}{0} \frac{0}{2} \frac{0}{1} \frac{0}{4} \frac{0}{3} \frac{0}{6} \frac{0}{5} \frac{0}{7} \frac{0}{8} \| \frac{1}{0} \frac{1}{3} \frac{6}{5} \frac{7}{0} \frac{7}{6} \frac{7}{7}$ & 711 & 1 & $4 A_{1}$ & $2 A_{1}+A_{3}$ & 3 & 6 & $\frac{0}{0} \frac{0}{2} \frac{0}{1} \frac{0}{4} \frac{0}{3} \frac{0}{6} \frac{0}{5} \frac{0}{8} \frac{0}{7} \| \frac{9}{7} \frac{1}{0} \frac{1}{3} \frac{2}{2} \frac{3}{0}$ \\
\hline 656 & 1 & $3 A_{1}$ & $2 A_{3}$ & 5 & 9 & $\frac{0}{0} \frac{0}{2} \frac{0}{1} \frac{0}{4} \frac{0}{3} \frac{0}{6} \frac{0}{5} \frac{0}{7} \frac{0}{8} \| \frac{1}{0} \frac{1}{3} \frac{6}{1} \frac{6}{2} \frac{2}{8} \frac{2}{f}$ & 712 & 1 & $4 A_{1}$ & $2 \underline{A_{1}}+A_{3}$ & 3 & 4 & $\frac{0}{0} \frac{0}{2} \frac{0}{1} \frac{0}{4} \frac{0}{3} \frac{0}{6} \frac{0}{5} \frac{0}{8} \frac{0}{7} \| \frac{9}{7} \frac{1}{c} \frac{2}{f} \frac{1}{0} \frac{1}{3}$ \\
\hline 657 & 1 & $3 A_{1}$ & $2 A_{1}+\underline{A_{1}}+A_{3}$ & 7 & 12 & $\frac{0}{0} \frac{0}{2} \frac{0}{1} \frac{0}{4} \frac{0}{3} \frac{0}{6} \frac{0}{5} \frac{0}{7} \frac{0}{8} \| \frac{9}{7} \frac{6}{0} \frac{6}{3} \frac{2}{8} \frac{3}{b} \frac{3}{c}$ & 713 & 1 & $4 A_{1}$ & $2 A_{1}+\underline{A_{1}}+A_{2}$ & 4 & 8 & $\frac{0}{0} \frac{0}{2} \frac{0}{1} \frac{0}{4} \frac{0}{3} \frac{0}{6} \frac{0}{5} \frac{0}{8} \frac{0}{7} \| \frac{9}{7} \frac{1}{0} \frac{1}{3} \frac{6}{1} \frac{7}{7}$ \\
\hline 658 & 1 & $3 A_{1}$ & $3 \underline{A_{1}}+A_{3}$ & 5 & 8 & $\frac{0}{0} \frac{0}{2} \frac{0}{1} \frac{0}{4} \frac{0}{3} \frac{0}{6} \frac{0}{5} \frac{0}{7} \frac{0}{8} \| \frac{9}{7} \frac{6}{0} \frac{6}{3} \frac{2}{8} \frac{2}{f} \frac{3}{e}$ & 714 & 1 & $4 A_{1}$ & $3 \underline{A_{1}}+A_{2}$ & 4 & 6 & $\frac{0}{0} \frac{0}{2} \frac{0}{1} \frac{0}{4} \frac{0}{3} \frac{0}{6} \frac{0}{5} \frac{0}{8} \frac{0}{7} \| \frac{9}{7} \frac{1}{c} \frac{2}{f} \frac{6}{7} \frac{8}{7}$ \\
\hline 659 & 1 & $3 A_{1}$ & $2 A_{1}+\underline{A_{1}}+\underline{A_{3}}$ & 3 & 6 & $\frac{0}{0} \frac{0}{2} \frac{0}{1} \frac{0}{4} \frac{0}{3} \frac{0}{6} \frac{0}{5} \frac{0}{7} \frac{0}{8} \| \frac{9}{7} \frac{5}{9} \frac{6}{4} \frac{2}{8} \frac{3}{a} \frac{3}{d}$ & 715 & 1 & $4 A_{1}$ & $4 A_{1}+\underline{A_{1}}$ & 4 & 7 & $\frac{0}{0} \frac{0}{2} \frac{0}{1} \frac{0}{4} \frac{0}{3} \frac{0}{6} \frac{0}{5} \frac{0}{8} \frac{0}{7} \| \frac{9}{7} \frac{1}{0} \frac{1}{3} \frac{3}{b} \frac{3}{c}$ \\
\hline 660 & 1 & $3 A_{1}$ & $2 A_{2}+A_{2}$ & 13 & 19 & $\frac{0}{0} \frac{0}{2} \frac{0}{1} \frac{0}{4} \frac{0}{3} \frac{0}{6} \frac{0}{5} \frac{0}{7} \frac{0}{8} \| \frac{4}{3} \frac{5}{3} \frac{6}{1} \frac{6}{2} \frac{8}{1} \frac{8}{4}$ & 716 & 1 & $4 A_{1}$ & $\underline{A_{1}}+2 A_{2}$ & 5 & 9 & $\frac{0}{0} \frac{0}{2} \frac{0}{1} \frac{0}{4} \frac{0}{3} \frac{0}{6} \frac{0}{5} \frac{0}{8} \frac{0}{7} \| \frac{9}{7} \frac{6}{0} \frac{6}{6} \frac{7}{8} \frac{8}{4}$ \\
\hline 661 & 1 & $3 A_{1}$ & $2 A_{2}+\underline{A_{2}}$ & 5 & 9 & $\frac{0}{0} \frac{0}{2} \frac{0}{1} \frac{0}{4} \frac{0}{3} \frac{0}{6} \frac{0}{5} \frac{0}{7} \frac{0}{8} \| \frac{5}{9} \frac{6}{7} \frac{7}{2} \frac{7}{9} \frac{8}{0} \frac{8}{5}$ & 717 & 1 & $4 A_{1}$ & $\underline{A_{1}}+2 A_{2}$ & 3 & 5 & $\frac{0}{0} \frac{0}{2} \frac{0}{1} \frac{0}{4} \frac{0}{3} \frac{0}{6} \frac{0}{5} \frac{0}{8} \frac{0}{7} \| \frac{9}{7} \frac{1}{0} \frac{1}{3} \frac{1}{4} \frac{1}{9}$ \\
\hline 662 & 1 & $3 A_{1}$ & $2 A_{1}+2 \underline{A_{1}}+A_{2}$ & 8 & 15 & $\frac{0}{0} \frac{0}{2} \frac{0}{1} \frac{0}{4} \frac{0}{3} \frac{0}{6} \frac{0}{5} \frac{0}{7} \frac{0}{8} \| \frac{4}{3} \frac{5}{3} \frac{2}{8} \frac{1}{c} \frac{3}{b} \frac{3}{c}$ & 718 & 1 & $4 A_{1}$ & $\underline{A_{1}}+A_{4}$ & 4 & 6 & $\frac{0}{0} \frac{0}{2} \frac{0}{1} \frac{0}{4} \frac{0}{3} \frac{0}{6} \frac{0}{5} \frac{0}{8} \frac{0}{7} \| \frac{9}{7} \frac{1}{0} \frac{1}{3} \frac{6}{5} \frac{8}{5}$ \\
\hline 663 & 1 & $3 A_{1}$ & $4 \underline{A_{1}}+\underline{A_{2}}$ & 6 & 11 & $\frac{0}{0} \frac{0}{2} \frac{0}{1} \frac{0}{4} \frac{0}{3} \frac{0}{6} \frac{0}{5} \frac{0}{7} \frac{0}{8} \| \frac{4}{3} \frac{5}{3} \frac{2}{8} \frac{1}{c} \frac{2}{f} \frac{3}{e}$ & 719 & 1 & $4 A_{1}$ & $A_{2}+A_{3}$ & 4 & 6 & $\frac{0}{0} \frac{0}{2} \frac{0}{1} \frac{0}{4} \frac{0}{3} \frac{0}{6} \frac{0}{5} \frac{0}{8} \frac{0}{7} \| \frac{9}{7} \frac{2}{2} \frac{3}{0} \frac{6}{9} \frac{8}{1}$ \\
\hline 664 & 1 & $3 A_{1}$ & $4 A_{1}+2 \underline{A_{1}}$ & 9 & 15 & $\frac{0}{0} \frac{0}{2} \frac{0}{1} \frac{0}{4} \frac{0}{3} \frac{0}{6} \frac{0}{5} \frac{0}{7} \frac{0}{8} \| \frac{9}{7} \frac{5}{9} \frac{7}{5} \frac{7}{8} \frac{3}{b} \frac{3}{c}$ & 720 & 1 & $4 A_{1}$ & $A_{5}$ & 3 & 3 & $\frac{0}{0} \frac{0}{2} \frac{0}{1} \frac{0}{4} \frac{0}{3} \frac{0}{6} \frac{0}{5} \frac{0}{8} \frac{0}{7} \| \frac{9}{7} \frac{1}{0} \frac{1}{3} \frac{2}{4} \frac{3}{2}$ \\
\hline 665 & 1 & $3 A_{1}$ & $4 A_{1}+2 \underline{A_{1}}$ & 7 & 15 & $\frac{0}{0} \frac{0}{2} \frac{0}{1} \frac{0}{4} \frac{0}{3} \frac{0}{6} \frac{0}{5} \frac{0}{7} \frac{0}{8} \| \frac{6}{0} \frac{6}{3} \frac{7}{4} \frac{7}{9} \frac{3}{b} \frac{3}{c}$ & 721 & 1 & $4 A_{1}$ & $2 \underline{A_{1}}+2 \underline{A_{2}}$ & 4 & 6 & $\frac{0}{0} \frac{0}{2} \frac{0}{1} \frac{0}{4} \frac{0}{3} \frac{0}{6} \frac{0}{5} \frac{0}{8} \frac{0}{7} \| \frac{9}{7} \frac{1}{c} \frac{6}{5} \frac{7}{1} \frac{8}{3} \frac{8}{5}$ \\
\hline 666 & 1 & $3 A_{1}$ & $\underline{A_{1}}+\ldots A_{2}+\ldots A_{3}$ & 7 & 12 & $\frac{0}{0} \frac{0}{2} \frac{0}{1} \frac{0}{4} \frac{0}{3} \frac{0}{6} \frac{0}{5} \frac{0}{7} \frac{0}{8} \| \frac{4}{3} \frac{5}{3} \frac{6}{1} \frac{6}{2} \frac{2}{8}$ & 722 & 1 & $4 A_{1}$ & $2 A_{1}+A_{4}$ & 3 & 6 & $\frac{0}{0} \frac{0}{2} \frac{0}{1} \frac{0}{4} \frac{0}{3} \frac{0}{6} \frac{0}{5} \frac{0}{8} \frac{0}{7} \| \frac{1}{0} \frac{1}{3} \frac{2}{2} \frac{3}{4} \frac{4}{2}$ \\
\hline
\end{tabular}




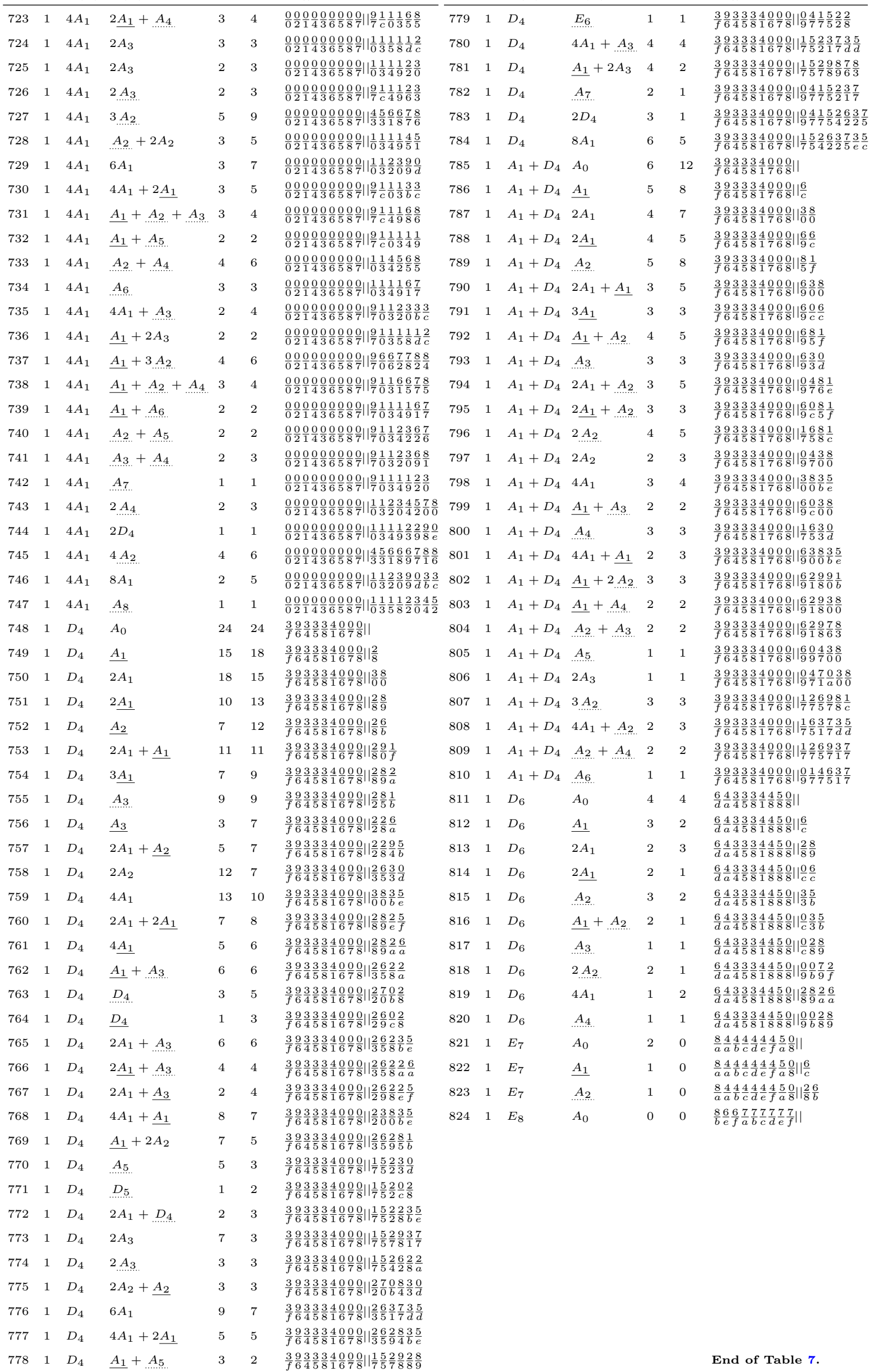




\section{Acknowledgements}

I would like to thank H. Pottmann and M. Skopenkov for interesting comments and discussions. The image of Figure 2 is adapted from [34, Wikipedia]. The images of surfaces were made using [23, Povray] and the algorithms were implemented using [31, Sage]. Financial support was provided by the Austrian Science Fund (FWF): P33003.

\section{References}

[1] A. Akopyan. 3-Webs generated by confocal conics and circles. Geom. Dedicata, 194:55-64, 2018.

[2] W. Blaschke and G. Bol. Geometrie der Gewebe. Topologische Fragen der Differentialgeometrie. J. W. Edwards, 1944.

[3] R. Blum. Circles on surfaces in the Euclidean 3-space. volume 792 of Lecture Notes in Math., pages 213-221. Springer, 1980.

[4] A. Cayley. On the triple tangent planes of surfaces of the third order. Cambridge and Dublin Math. J., 4:118-138, 1848.

[5] A. Clebsch. Über die Anwendung der quadratischen Substitution auf die Gleichungen 5ten Grades und die geometrische Theorie des ebenen Fünfseits. Math. Ann., 4:284-345, 1871.

[6] I. V. Dolgachev. Classical algebraic geometry: A modern view. Cambridge University Press, 2012. ISBN 978-1-107-01765-8.

[7] P. Du Val. On isolated singularities of surfaces which do not affect the conditions of adjunction. i, ii, iii. Proc. Cambridge Phil. Soc., 30, 1934.

[8] D. Fuchs and S. Tabachnikov. Mathematical omnibus: Thirty lectures on classical mathematics. Amer. Math. Soc., 2007.

[9] H. Graf and R. Sauer. Über dreifache Geradensysteme. Math. nat. Abt., pages 119-156, 1924.

[10] B. Green and T. Tao. On sets defining few ordinary lines. Discrete $\mathscr{G}$ Computational Geometry, 50(2):409-468, 2013. 
[11] B. Hall. Lie groups, Lie algebras, and representations, volume 222 of Graduate Texts in Mathematics. Springer, 2015.

[12] R. Hartshorne. Algebraic geometry. Springer-Verlag, 1977.

[13] H. Knörrer and T. Miller. Topologische Typen reeller kubischer Flächen. Math. Z., 195:51-67, 1987.

[14] J. Kollár. Real algebraic surfaces. arXiv:alg-geom/9712003, 1997.

[15] J. Kollár. Lectures on resolution of singularities, volume 166. Princeton University Press, 2007. ISBN 978-0-691-12923-5.

[16] N. Lubbes. Sage library for computations in Neron-Severi lattice, 2018. github.com/niels-lubbes/ns_lattice.

[17] N. Lubbes. Sage library for constructing and visualizing curves on surfaces., 2018. github.com/niels-lubbes/orbital.

[18] N. Lubbes. Minimal degree rational curves on real surfaces. Adv. Math., 345: 263-288, 2019.

[19] Y. I. Manin. Cubic forms. North-Holland Publishing Co., 1986. ISBN 0-44487823-8.

[20] Mathoverflow, 2014. mathoverflow.net/questions/172550/graphs-of-lines-ondel-pezzo-surfaces.

[21] F. Nilov. On new constructions in the Blaschke-Bol problem. Sb. Math., 205: 1650-1667, 2014.

[22] J. V. Pereira and L. Pirio. An invitation to web geometry, volume 2 of IMPA Monographs. Springer, 2015. ISBN 978-3-319-14561-7.

[23] Povray. Persistence of Vision Pty. Ltd., 2004. povray.org.

[24] I. Polo-Blanco and J. Top. Explicit real cubic surfaces. Canad. Math. Bull., 51 (1):125-133, 2008.

[25] H. Pottmann, L. Shi, and M. Skopenkov. Darboux cyclides and webs from circles. Comput. Aided Geom. Design, 29(1):77-97, 2012. 
[26] Q. Ren, K. Shaw, and B. Sturmfels. Tropicalization of del Pezzo surfaces. Adv. Math., 300:156-189, 2016.

[27] J. Schicho. The multiple conical surfaces. Beitr. Alg. Geom., 42:71-87, 2001.

[28] J. Schicho. Elementary theory of del Pezzo surfaces. In Computational methods for algebraic spline surfaces, pages 77-94. Springer, 2005.

[29] A. M. Shelekhov. Classification of regular three-webs formed by pencils of circles. J. Math. Sciences., 2007.

[30] R. Silhol. Real algebraic surfaces, volume 1392 of Lecture Notes in Mathematics. Springer, 1989.

[31] W. A. Stein et al. Sage Mathematics Software. The Sage Development Team, 2012. sagemath.org.

[32] Y. Villarceau. Theoreme sur le tore. Nouvelles annales de mathematiques, 7: 345-347, 1848. eudml.org/doc/95880.

[33] C. T. C. Wall. Real forms of smooth del Pezzo surfaces. J. Reine Angew. Math., 375/376:47-66, 1987.

[34] Wikipedia, 2018. en.wikipedia.org/wiki/Generalized_quadrangle.

[35] C. Wren. Generatio corporis cylindroidis hyperbolici, elaborandis lenti bus hyperbolicis accommodati. 4:961-962, 1669. doi.org/10.1098/rstl.1669.0018.

address of author: Johann Radon Institute for Computational and Applied Mathematics (RICAM), Austrian Academy of Sciences

email: niels.lubbes@gmail.com 